\title{
EVALUATION OF HUMAN UMBILICAL VEIN ENDOTHELIAL CELLS IN BLOOD VESSEL MIMICS THROUGH CHANGES IN GENE EXPRESSION AND CASPASE ACTIVITY
}

\author{
A thesis \\ presented to \\ the Faculty of California Polytechnic State University, \\ San Luis Obispo \\ In Partial Fulfillment \\ of the Requirements for the Degree \\ Master of Science in Biomedical Engineering
}

by

Conor Charles Hedigan

June 2019 
(C) 2019

Conor Charles Hedigan

ALL RIGHTS RESERVED 


\section{COMMITTEE MEMBERSHIP}

TITLE: Evaluation of Human Umbilical Vein

Endothelial Cells in Blood Vessel Mimics

through Changes in Gene Expression and

Caspase Activity

AUTHOR: Conor Charles Hedigan

DATE SUBMITTED: June 2019

COMMITTEE CHAIR: Kristen O'Halloran Cardinal, Ph.D.

Professor of Biomedical Engineering

COMMITTEE MEMBER: Trevor Ryan Cardinal, Ph.D.

Professor of Biomedical Engineering

COMMITTEE MEMBER: Lily Laiho, Ph.D.

Professor of Biomedical Engineering 


\begin{abstract}
Evaluation of Human Umbilical Vein Endothelial Cells in Blood Vessel Mimics through Changes in Gene Expression and Caspase Activity

Conor Charles Hedigan
\end{abstract}

Blood vessel mimics (BVMs) are simple tissue engineered blood vessel constructs intended for preclinical testing of vascular devices. This thesis developed and implemented methods to characterize two of these components. The first aim of this thesis investigated the effect of cell culture duration and flow conditions on endothelial cell gene expression, especially regarding endothelial-to-mesenchymal transition (EndMT). A trend of decreased endothelial marker gene expression and increased mesenchymal marker gene expression would indicate EndMT. qPCR analysis revealed that increased cell culture duration did not result in EndMT, and in fact increased endothelial marker expression as cell culture duration increased. Disturbed flow conditions decreased endothelial marker and increased mesenchymal marker expression relative to static culture.

The second aim of this thesis developed methods to determine cytotoxicity of, and endothelial cell adhesion to, novel BTEAC salt scaffolds. Immunostaining was used to visualize these scaffold effects. The cytotoxicity elution assay showed that BTEAC salt scaffolds were not more cytotoxic than the standard PLGA scaffold. Direct contact assays spanning several timepoints also found that BTEAC salt scaffolds were not more cytotoxic than standard scaffolds but had higher endothelial cell adhesion and coverage than standard scaffolds. Overall, this thesis developed and implemented methods to characterize the endothelial cells used in the BVM model. 


\section{ACKNOWLEDGMENTS}

I would like to thank my advisor, Dr. Kristen Cardinal, for her support throughout my undergraduate and graduate studies. The investment she makes in each student, including myself, is remarkable and unprecedented. I cannot imagine my college experience without the knowledge and passion for innovation that she imparts in the laboratory and classroom. Furthermore, I would like to thank her for her dedication to her role as a thesis advisor, with which her countless hours of guidance did not go unnoticed and I will always be appreciative of.

I would like to thank my committee members, Dr. Trevor Cardinal and Dr. Lily Laiho, for their time and effort to enhance my thesis. Fellow laboratory research members contributed to the efforts of this thesis as well, and for that I would like to acknowledge and thank Ryan Murphy, Ali Flesch, and Cami Dozois. I would also like to thank Ella Sugerman and Ben Dennis for their assistance in the laboratory and willingness to share their knowledge to promote my success.

Finally, I would like to thank my family and friends for their support throughout my years in San Luis Obispo. 


\section{TABLE OF CONTENTS}

Page

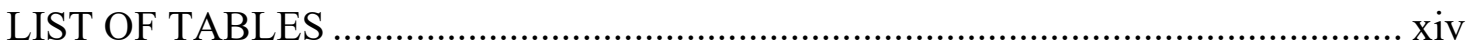

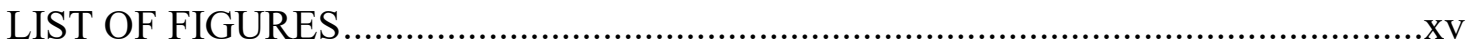

LIST OF EQUATIONS ……………………………....................................... xxi

\section{CHAPTER}

1. INTRODUCTION ……………………………………………………………………1

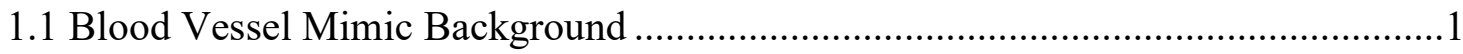

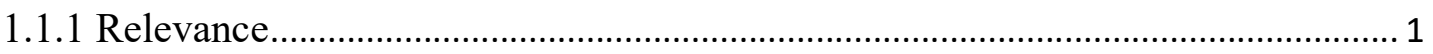

1.1.1.1 Cardiovascular Disease and Intravascular Devices .....................................1

1.1.1.2 Preclinical Cardiovascular Device Testing in Blood Vessel Mimics ...........2

1.1.2 Blood Vessel Mimic Design .............................................................................

1.1.2.1 Native Blood Vessel Anatomy........................................................................

1.1.2.2 Blood Vessel Mimic Fabrication ............................................................

1.1.2.3 Blood Vessel Mimic Anatomy.....................................................................

1.1.3 Types of Blood Vessel Mimics.......................................................................... 6

1.1.4 Importance of Endothelial Cell Characterization ................................................

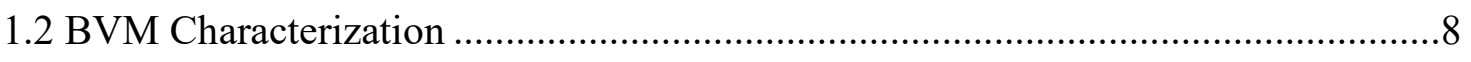

1.2.1 Previous Characterization............................................................................

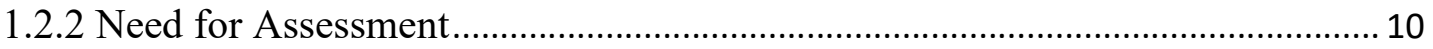


1.3 Summary and Aims of the Thesis .12

1.3.1 Aim 1: EC Characterization through Changes in Gene Expression 12

1.3.2 Aim 2: Characterization of Endothelial Cell and Scaffold Interaction 13

\section{AIM 1: GENE EXPRESSION CHANGES OF ENDOTHELIAL CELLS IN} CULTURE AS A FACTOR OF TIME AND FLOW CONDITION.

2.1 Introduction 14

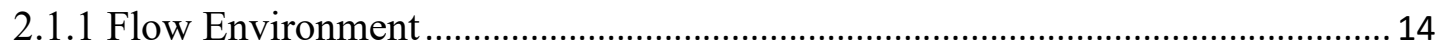

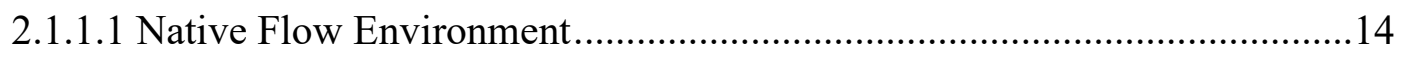

2.1.1.2 Previously Determined Flow Conditions in Blood Vessel Mimics .............17

2.1.2 BVM Cell Phenotype ………………………………………………………... 19

2.1.2.1 Immunostaining of HUVECs in the Tissue Engineering Lab ...................19

2.1.2.2 Endothelial-to-Mesenchymal Transition ..............................................20

2.1.2.2.1 EndMT in Heart Development ..........................................................21

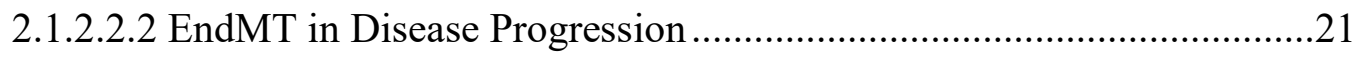

2.1.2.2.3 EndMT in Tissue Regeneration ....................................................22

2.1.2.3 EndMT Activation Pathways .................................................................24

2.1.2.3.1 Transforming Growth Factor Beta ……………………………….......24

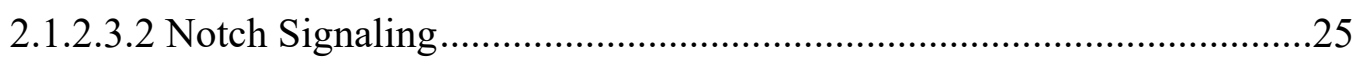

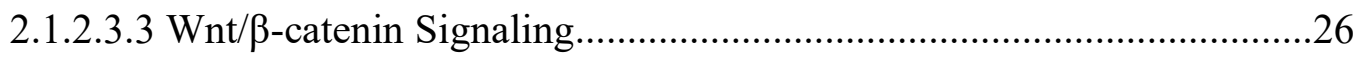


2.1.2.3.4 Pro-inflammatory Cytokines .26

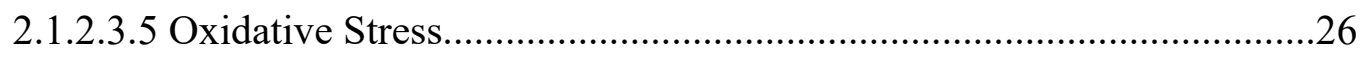

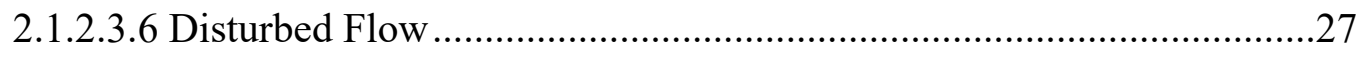

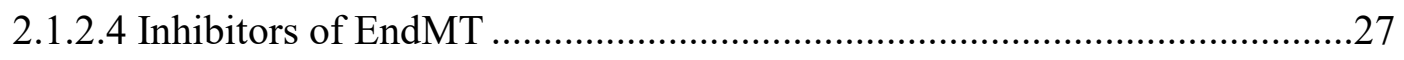

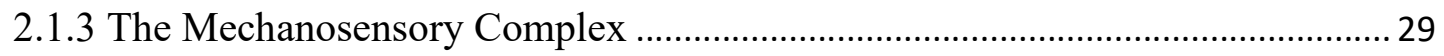

2.1.3.1 Platelet Endothelial Cell Adhesion Molecule 1 (PECAM-1) ......................30

2.1.3.2 Vascular Endothelial Cadherin (VE-Cad) ……….....................................32

2.1.3.3 Vascular Endothelial Growth Factor Receptor 2 (VEGFR2) ......................33

2.1.3.4 VE-Cadherin Regulation of VEGFR2 …………..........................................

2.1.3.5 Mechanosensory Complex and EndMT....................................................36

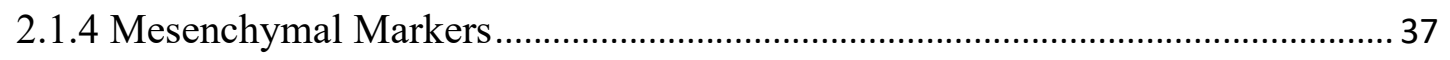

2.1.5 Using EC and Mesenchymal Markers to Determine EndMT Progression ......... 39

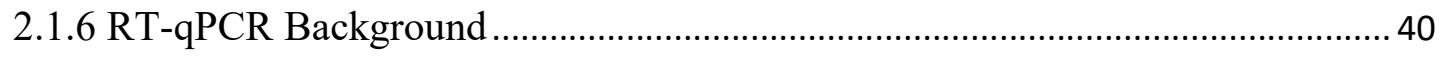

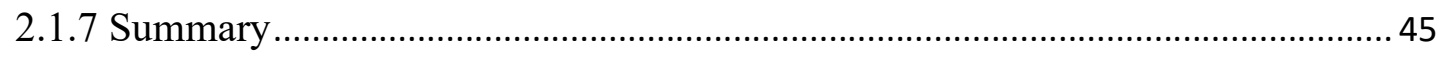

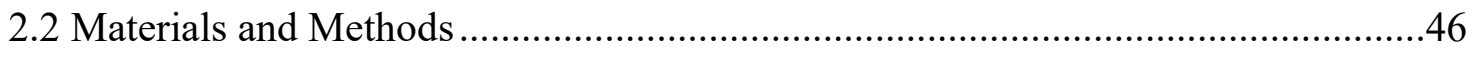

2.2.1 Gene Selection and Primer Acquisition................................................................ 46

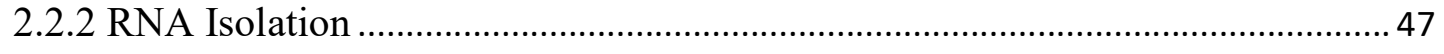

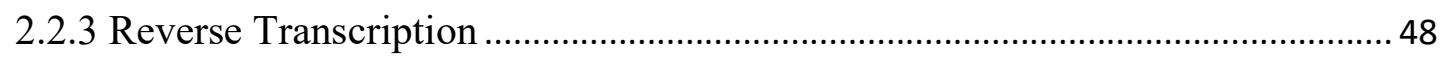


2.2.4 Quantitative Polymerase Chain Reaction. .49

2.2.5 Experiment 1 Pilot: Change in EC Expression with Increasing Passage ............ 50

2.2.6 Experiment 1: Change in EC Expression with Increasing Passage Number ..... 51

2.2.7 Experiment 2: Changes in EC Expression from Disturbed Flow Conditions ... 51

2.2.8 Experiment 3 Preparation: RNA Isolation Protocol for BVMs. .52

2.2.9 Experiment 3 Pilot: Changes in Gene Expression in BVMs 53

2.3 Results .54

2.3.1 Primer Efficiency. 54

2.3.2 Experiment 1 Pilot Results 55

2.3.3 Experiment 1 Results .59

2.3.4 Experiment 2 Results .64

2.3.5 Verification of RNA Isolation Protocol for BVMs. 67

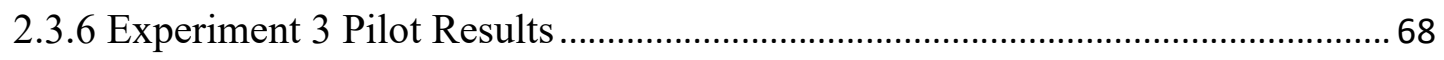

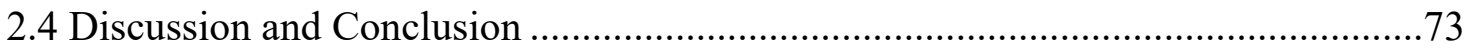

2.4.1 Experiment 1 Pilot and Experiment 1 ............................................................... 73

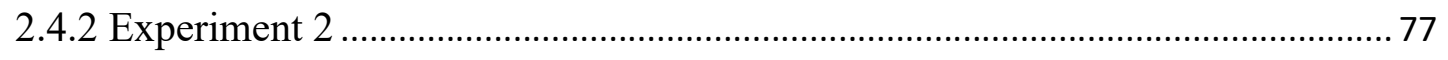

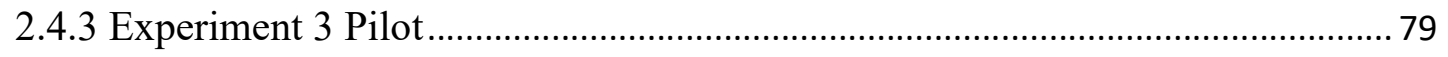

2.4.4 Aim 1: Limitations and Future Work ………………………………………... 83 
3. AIM 2: CHARACTERIZATION OF NOVEL SCAFFOLDS THROUGH HUVEC CASPASE ACTIVITY AND SCAFFOLD COVERAGE .86

3.1 Introduction 86

3.1.1 Scaffold Effects on Cells 86

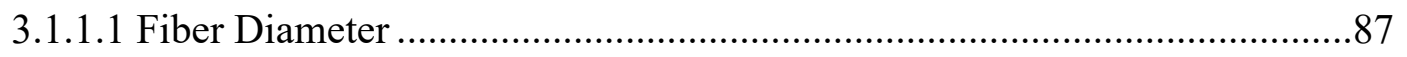

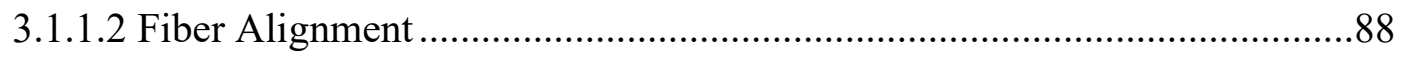

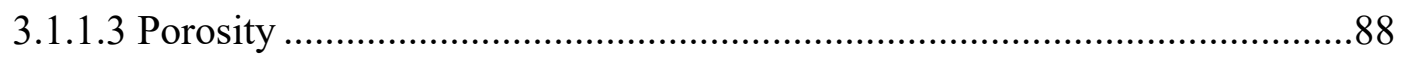

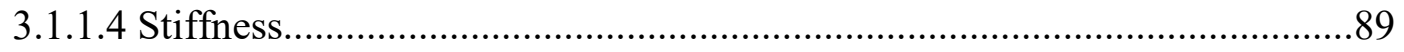

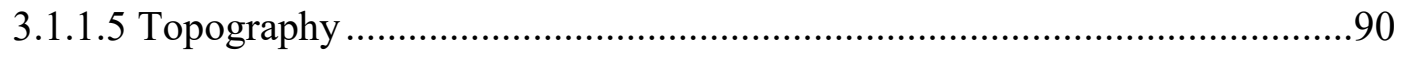

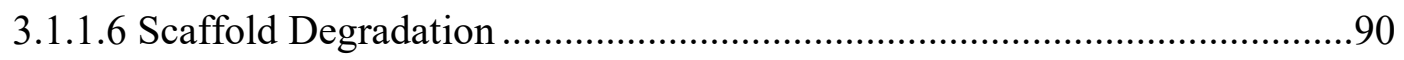

3.1.2 Measurement of Cell Responses ………………………………………….... 91

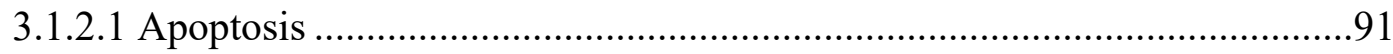

3.1.2.1.1 Apoptosis Pathways …………........................................................99

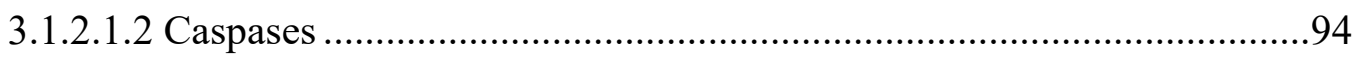

3.1.2.1.3 Apoptosis Detection and Quantification ................................................94

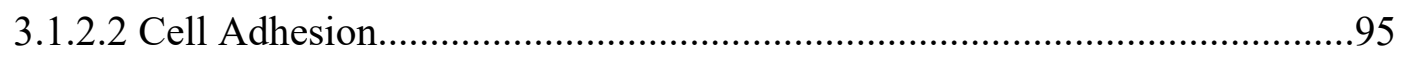

3.1.2.2.1 Passive In Vitro Cell Adhesion .............................................................95

3.1.2.2.2 Cell Adhesion Tests ......................................................................97 


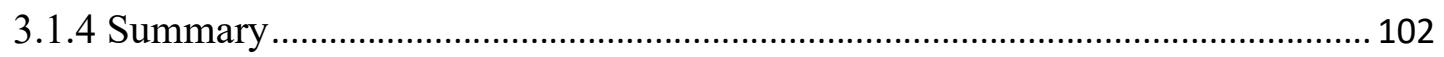

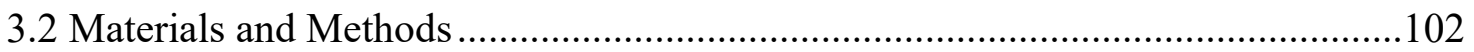

3.2.1 Caspase Stain Implementation and Verification ............................................ 103

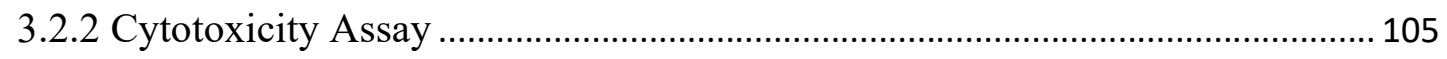

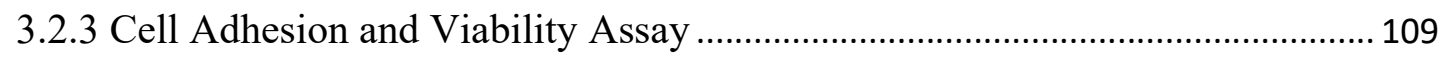

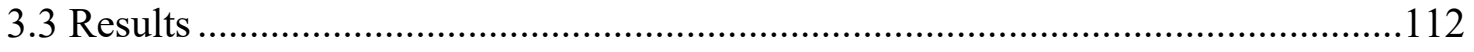

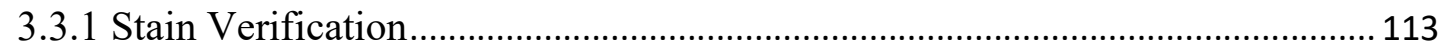

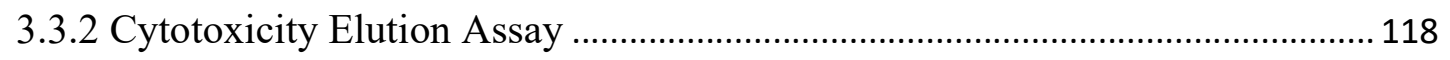

3.3.3 Cell Adhesion and Viability Assay ................................................................ 126

3.3.4 BVM Cell Coverage and Viability Assay ...................................................... 133

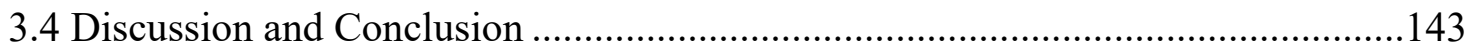

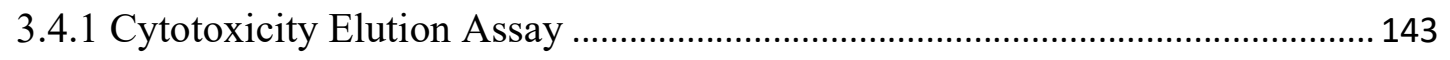

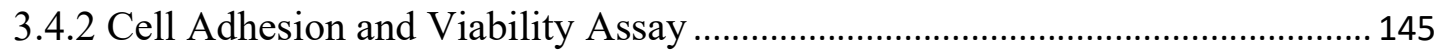

3.4.3 BVM Cell Coverage and Viability Assay …................................................... 146

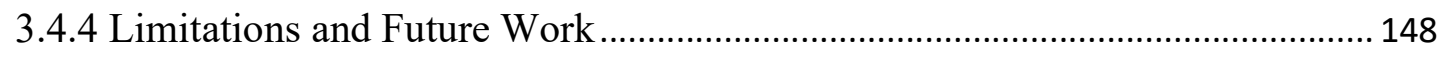

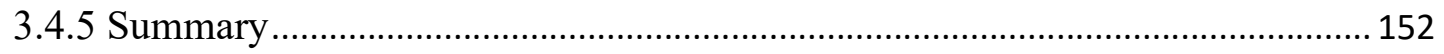

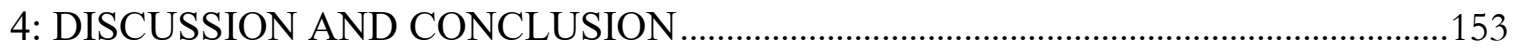

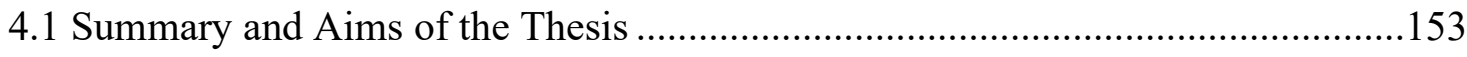


4.2 Results and Discussion

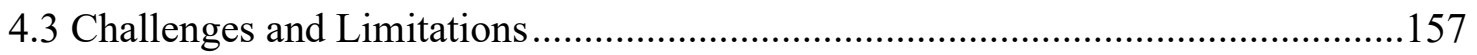

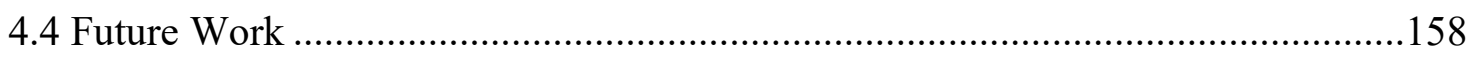

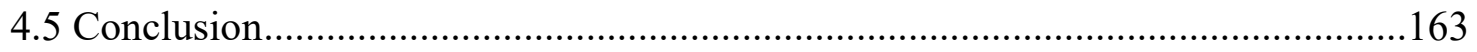

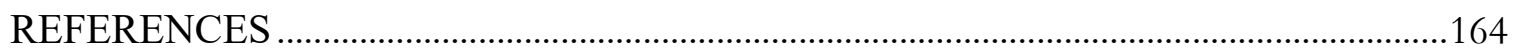

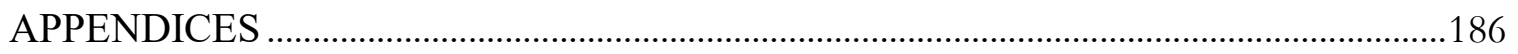

Appendix A: Preverified Primer Sequences and Characteristics ..............................186

Appendix B: Primer Efficiency Testing SOP ....................................................... 188

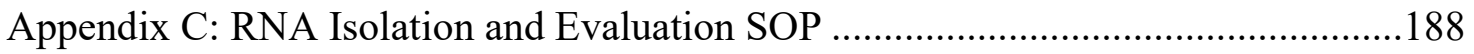

Appendix D: cDNA through Reverse Transcription SOP ......................................188

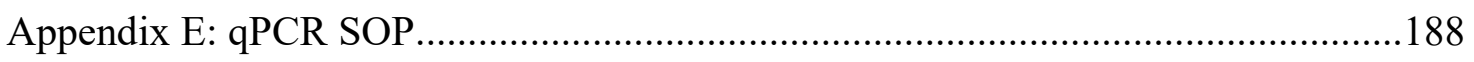

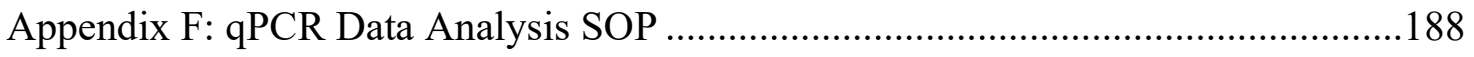

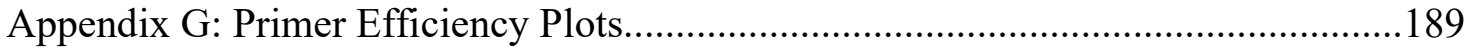

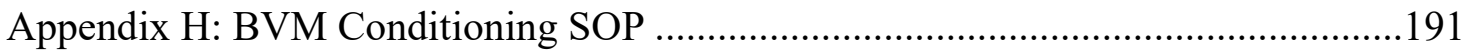

Appendix I: Endothelial Cell-only BVM Sodding SOP ......................................191

Appendix J: Apoptosis Kit Staining SOP ......................................................191

Appendix K: Cytotoxicity Elution Assay SOP ....................................................191

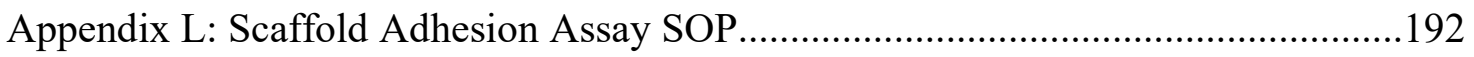


Appendix M: Positive Control Test Representative Images

Appendix N: Cytotoxicity Elution Assay Representative Images ..............................197

Appendix O: Scaffold Adhesion Assay Representative Images ................................203

Appendix P: BVM Study Representative Images ................................................207

Appendix Q: Magnification Justification Raw Data ..............................................213

Appendix R: Pixel Intensity Reduction Raw Data.............................................215

Appendix S: Nuclei Area Multiplication Factor Raw Data ...................................218

Appendix T: Statistical Tests for Cytotoxicity Elution Assay .................................219

Appendix U: Statistical Tests for Scaffold Adhesion Assay....................................223

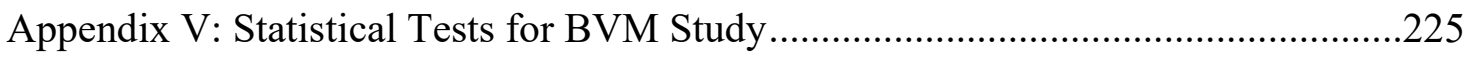




\section{LIST OF TABLES}

Table $\quad$ Page

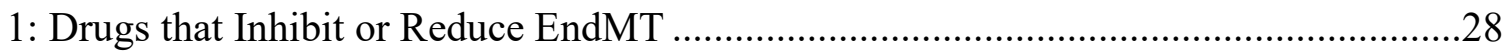

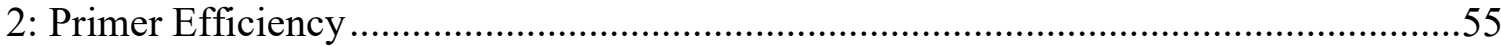

3: Comparison of RNA isolation from the RNeasy Mini Kit and Trizol extraction..........68

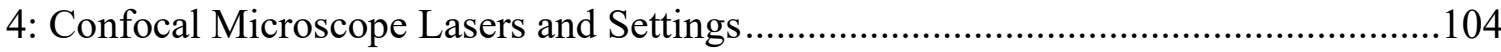

5: Apoptosis Staining Kit Verification using HUVECs ............................................113

6: $\mathrm{pH}$ of Elutions from Treatment Methods....................................................................120

7: Short-term Adhesion Assay Cell Sodding and Collection Data ...............................127 


\section{LIST OF FIGURES}

Figure $\quad$ Page

1: The Effects of Plaque on Blood Flow...................................................................

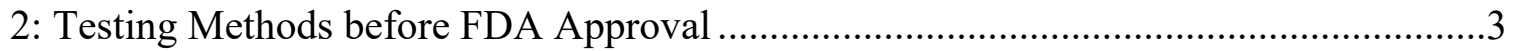

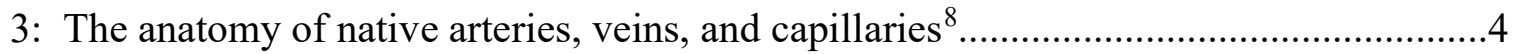

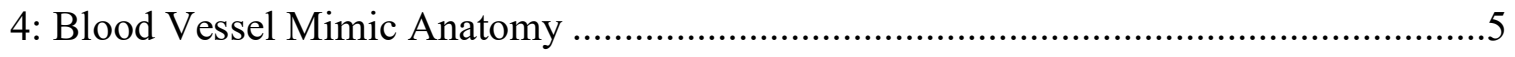

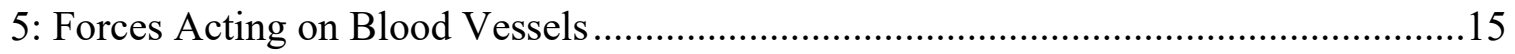

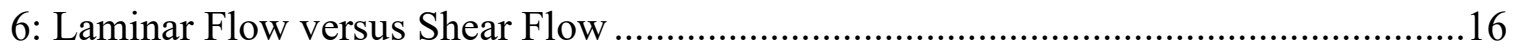

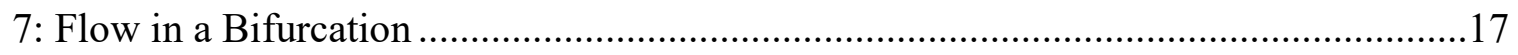

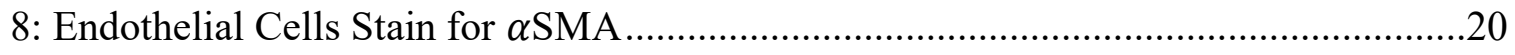

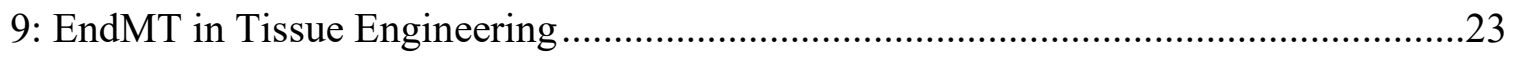

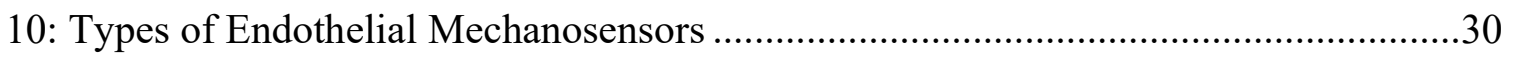

11: Mechanisms of VE-Cad and VEGFR2 on Cell Seeding Density ..............................35

12: VE-cad and Transgelin Expression in a Simple Flow Model....................................40

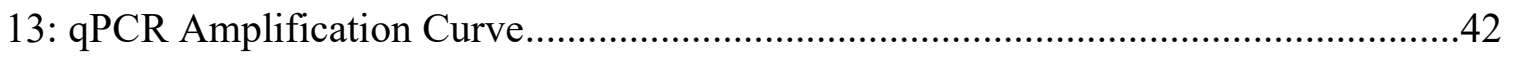

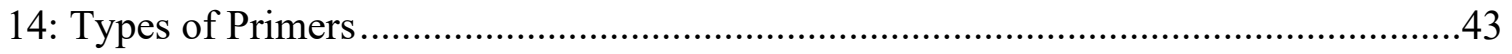

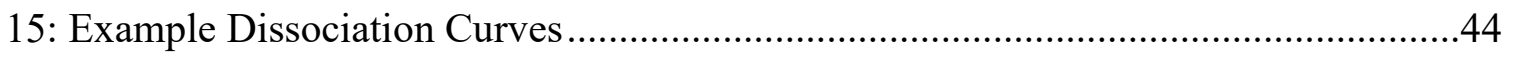


16: Primer Efficiency Plot

17: Experiment 1 Pilot Relative Abundance Relative to $\beta$-Actin

18: Experiment 1 Pilot Relative Abundance Relative to $\beta$ Galactosidase. .58

19: Experiment 1 Pilot Housekeeping Gene Stability .59

20: Experiment 1 Housekeeping Gene Stability 60

21: Experiment 1 Relative Abundance 61

22: Experiment 1 Pilot Relative Abundance of Mesenchymal Markers in SMCs .62

23: Representative dissociation curves of all GOI 63

24: Experiment 2 Relative Abundance. .66

25: Experiment 2 Housekeeping Gene Stability .67

26: HUVEC Morphology in Turbulent Flow Condition.

27: Experiment 3 Housekeeping Gene Stability .70

28: Disturbed Flow Dissociation Curves .71

29: Experiment 3 Relative Abundance .72

30: Dissociation Curves for GOI for EC-only BVMs .81

31: Cell Interactions with Fibers of Varying Sizes. 87 
32: The Apoptosis Pathway

33: Cell Adhesion Phases

.96

34: SEM of Standard Scaffold

35: SEM of Salt Scaffold 99

36: Fiber Diameter Reduction with BTEAC Percentage 100

37: Hoechst Channel Automatic Cell Counting 106

38: Pixel Reduction in Caspase Channel 108

39: Pixel Reduction in Propidium Iodide Channel 108

40: Hoechst Channel Coverage Quantification

41: Ineffective Positive Control DMSO 15\% 30 Minutes 116

42: Ineffective Positive Control Hydrogen Peroxide 1\% 2 Minutes 116

43: Effective Positive Control Hydrogen Peroxide 0.5\% 5 Minutes

44: Effective Positive Control Hydrogen Peroxide 1\% 5 Minutes

45: Scaffolds After Elution Treatment. 119

46: Media After Elution Treatment

47: Cell Count Reduction Curves

48: Cytotoxicity Elution Assay Percent Caspase Positive Cells 123 xvii 
49: Normalized Cytotoxicity Elution Assay Percent Caspase Positive Cells

50: Cytotoxicity Elution Assay Percent Propidium Iodide Positive Cells .125

51: Normalized Cytotoxicity Elution Assay Percent Caspase Positive Cells

52: Sample 20x HUVECs. 128

53: Standard and Salt Scaffolds Hoechst Channel. .129

54: Short-term Adhesion Assay Percent Cell Coverage..... .130

55: Caspase and Propidium Iodide Channels Short-term Adhesion Assay

56: Short-term Adhesion Assay Percent Apoptotic Cells. .132

57: Short-term Adhesion Assay Percent Dead Cells .132

58: Salt Scaffold Shortening Before and After BVM Cultivation .134

59: Standard and Salt Scaffold Shortening After BVM Cultivation. .134

60: Wall Thicknesses of Scaffolds in BVMs .135

61: Representative 3-day BVM Hoechst Images .136

62: Representative 7-day BVM Hoechst Images .137

63: BVM Cell Coverage in 3 and 7-Day BVMs.... .138

64: Images of 3-day BVM Caspase and Propidium Iodide Channels .140

65: Images of 7-day BVM Caspase and Propidium Iodide Channels .141 xviii 
66: Percent Apoptotic Cells in 3 and 7-Day BVMs...

67: Percent Dead Cells in 3 and 7-Day BVMs 143

68: Calponin immunostaining of HUVECs and HUASMCs...........................................160 


\section{List of Equations}

Equation

Page

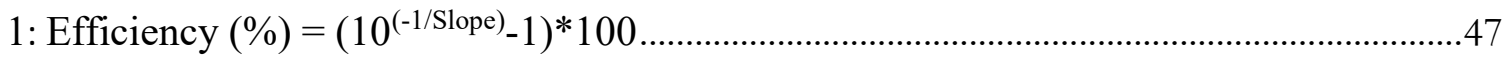

2: $(1000 \mathrm{ng} \mathrm{RNA}) *(1 \mathrm{~mL} /[\mathrm{RNA}$ concentration $] \mathrm{ng} / \mathrm{nL})=[$ Volume $\mathrm{RNA}] \mu \mathrm{L} \ldots \ldots \ldots \ldots \ldots \ldots . . . .49$

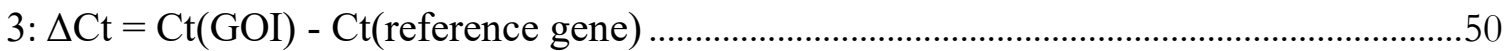




\section{CHAPTER 1. INTRODUCTION}

\subsection{Blood Vessel Mimic Background}

Cal Poly's Tissue Engineering Laboratory creates blood vessel mimics (BVMs) to test intravascular devices in an environment that simplistically mimics the anatomy and physiology of native blood vessels. The BVMs are fabricated using endothelial cells (ECs), smooth muscle cells (SMCs), and a polymer scaffold tube assembled in a bioreactor. While this design is simple, it offers a three-dimensional environment with some physiologic relevance, which can be used to determine the interaction between cells and vascular devices. This model serves as an intermediate step between basic in vitro testing and in vivo testing of devices. A full explanation of BVM relevance for cardiovascular device testing will be explained in the next section.

\subsubsection{Relevance}

\subsubsection{Cardiovascular Disease and Intravascular Devices}

Heart disease is responsible for one of every four deaths per year in the United States, with coronary artery disease (CAD) being the most common type of heart disease $^{1}$. Coronary heart disease results in the hardening and thickening of coronary arteries and the formation of atherosclerotic plaques. These plaques are composed of immune cells, SMCs, myofibroblasts, lipids, and extracellular matrix, and develop preferentially at areas of disturbed flow in the vessels ${ }^{2}$. These plaques, impede blood delivery to the heart and are prone to rupturing, which can result in a myocardial 
infarction or stroke ${ }^{1}$. Figure 1 displays the progression of atherosclerotic plaque building and the resulting flow.
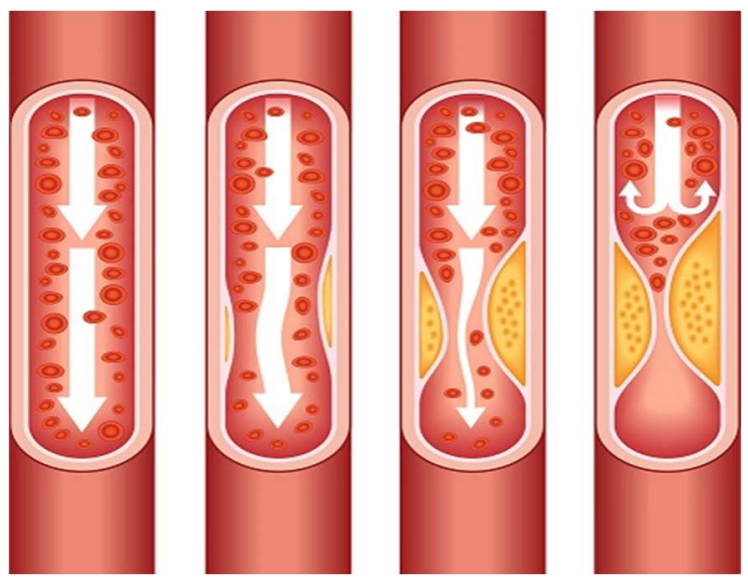

Figure 1: The Effects of Plaque on Blood Flow. Blood flow is impeded as atherosclerotic plaque buildup progresses ${ }^{1}$.

Intervention is often necessary to reduce the area of plaque blockage and increase blood flow to downstream tissue. Coronary artery bypass grafting $(\mathrm{CABG})$ is a procedure commonly used to bypass the occluded vessel and restore blood flow, but the procedure is invasive. Minimally invasive procedures include coronary balloon angioplasty and stenting. Coronary balloon angioplasty uses a balloon to push the plaque against the wall of the vessel, widening the lumen of the vessel ${ }^{3}$. Stenting restores blood flow, similar to coronary balloon angioplasty, but leaves a stent in the artery.

\subsubsection{Preclinical Cardiovascular Device Testing in Blood Vessel Mimics}

The BVM model is intended as an intermediate testing environment for intravascular devices, such as coronary stents, to support safety of the device. These types of devices must show safety and efficacy in vitro, in vivo, and during clinical trials. Most in vitro testing simply uses cells cultured in a two-dimensional plate to determine cell 
interaction with the device material. Testing then progresses to in vivo studies, where devices are tested in animal models. There is a disparity between how cells interact in a plate with a device and how a living organism interacts with that device. In vivo studies are also expensive, so alternative testing environments can be useful in determining safety as well as reducing testing costs. BVMs can be used as a testing environment to bridge current in vitro and in vivo testing, which is shown in Figure 2.

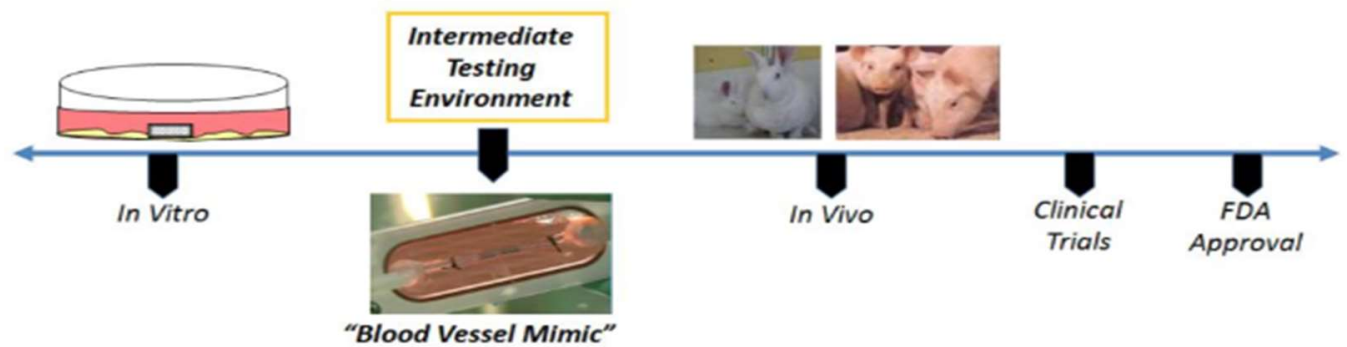

Figure 2: Testing Methods before FDA Approval. BVMs can provide valuable data on device safety and efficacy between in vitro and in vivo testing ${ }^{4}$.

\subsubsection{Blood Vessel Mimic Design}

\subsubsection{Native Blood Vessel Anatomy}

The BVM model is designed to have a simpler anatomy than a native vessel. Different types of blood vessels vary slightly in anatomy but share three main tunica layers and a lumen. The lumen is an open tube at the center of the vessel and is the site of blood flow. The tunica intima is the innermost layer which interacts with blood in the lumen and is composed of the endothelium and basement membrane ${ }^{5}$. The endothelium is composed of ECs and acts as a barrier between the body and blood while being selectively permeable to chemicals, white blood cell, and waste movement ${ }^{6}$. The basement membrane anchors the endothelium to the connective tissue of the middle layer 
of blood vessel tissue, the tunica media. The tunica media contains smooth muscle cells and connective tissue and is responsible for vasoconstriction and vasodilation ${ }^{5}$. The outermost layer, the tunica adventitia, consists of collagenous connective tissue, fibroblasts, and nerves ${ }^{7}$. A diagram of these layers is shown in Figure 3. BVMs attempt to include the lumen, tunica intima, tunica media, and tunica adventitia.

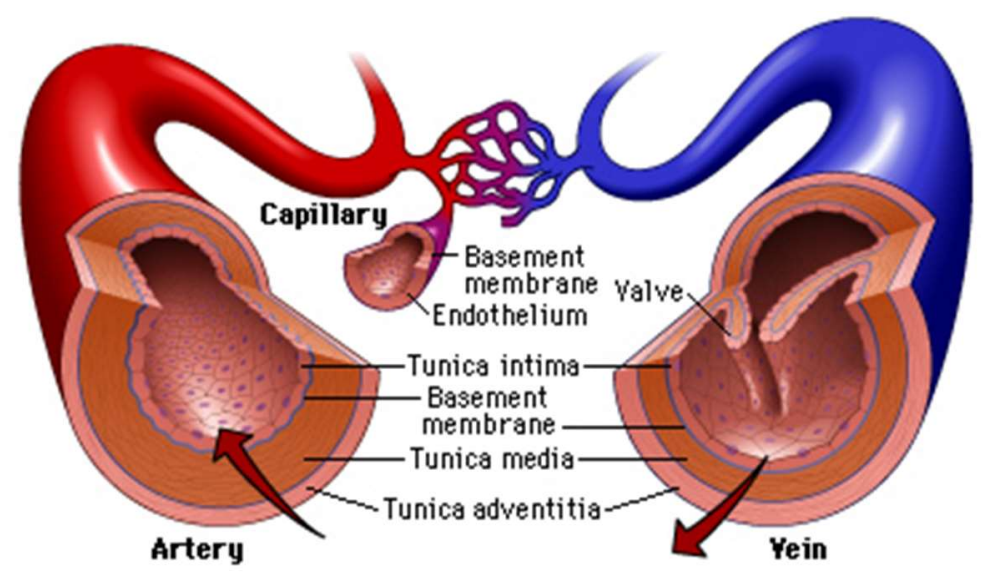

Figure 3: The anatomy of native arteries, veins, and capillaries ${ }^{8}$.

\subsubsection{Blood Vessel Mimic Fabrication}

BVMs are fabricated using three components: an exterior polymer scaffold tube, a middle layer of SMCs, and an interior layer of ECs ${ }^{9}$. The scaffold is composed of polylactic-co-glycolic-acid (PLGA) fibers, in which fibers are created through electrospinning. During the electrospinning process, a solution of chloroform and PLGA is charged and pulled to a grounded metal mandrel. The mandrel translates and rotates to collect the fibers, which typically range between $4 \mu \mathrm{m}$ to $5 \mu \mathrm{m}$ in fiber diameter.

The scaffold is then conditioned in high serum media within a bioreactor attached to a peristaltic pump, where proteins from the media intercalate the scaffold. These proteins enhance cell adhesion and proliferation once cells are sodded on the scaffold. 
With the current model, human umbilical artery smooth muscle cells (HUASMCs) are pressure sodded to the interior of the polymer tube, followed by human umbilical vein endothelial cells (HUVECs) after conditioning. The fully fabricated BVM is cultured inside of the bioreactor, which is connected to an 8-roller peristaltic pump that pushes media through the lumen of the BVM. After reaching the appropriate time point, the $\mathrm{BVM}$ is removed from the bioreactor and prepared for one of several techniques to analyze the resulting construct, including confocal imaging, scanning electron microscope (SEM) imaging, and gene expression analysis.

\subsubsection{Blood Vessel Mimic Anatomy}

Due to the fabrication technique, and as introduced above, the BVM has an anatomy mimicking the three basic layers of a native vessel. The polymer scaffold acts as the tunica adventitia of the BVM, supporting the geometry and providing a matrix for SMC growth. Figure 4 displays the layer of HUASMCs acting as the tunica media, and the layer of HUVECs acting as the tunica intima.

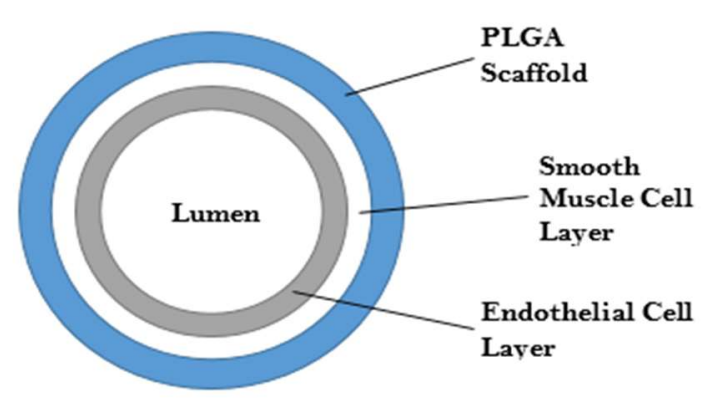

Figure 4: Blood Vessel Mimic Anatomy. 


\subsubsection{Types of Blood Vessel Mimics}

Cal Poly's Tissue Engineering Lab currently creates three different types of BVMs for use with different devices or diseases. The straight BVM is the standard model used to mimic a healthy blood vessel and has primarily been used for stent and intravascular imaging testing ${ }^{9}$. Aneurysm BVMs are fabricated to mimic the geometry of an aneurysm in a vesse ${ }^{10}$. Several aneurysm geometries have been created, including blister, fusiform, and saccular, which are useful for testing neurovascular devices such as flow diverters ${ }^{10}$. A diabetic BVM has also been developed, which uses diabetic cells instead of non-diseased ECs and SMCs ${ }^{11,12}$. Stenting in diabetic patients has a lower rate of success than in non-diabetic patients, so further testing of devices that will be used in diabetics may be important to understand safety concerns.

Although the examples described above incorporated both ECs and SMCs, BVMs using only endothelial cells have also been developed and implemented in the past. Cardinal et al. initially developed these BVMs using ePTFE scaffolds that were sodded with only human microvessel endothelial cells ${ }^{4}$. It was observed that cell coverage was significantly reduced upon the implementation of PLGA scaffolds in the BVM model, so SMCs were introduced to promote EC adhesion. While the addition of SMCs makes the model anatomically more similar, it also adds an increased amount of additional resources and time to create the model. The addition of SMCs also complicates analysis of the BVMs, requiring the ability to distinguish between cells types and understand the interactions of the two cell types. Therefore, it would be beneficial for the Tissue Engineering Lab to have the capability to create EC only BVMs. Analysis of cell-cell 
interactions, cell-scaffold interactions, and cell-device interactions in these BVMs would be simplified.

\subsubsection{Importance of Endothelial Cell Characterization}

Device testing has always been the goal for the BVM model. The interaction between the device and endothelial cells is the primary consideration for analysis since this cell-device interaction is the dominant interaction in vivo; therefore, the endothelial cells used in the model are of utmost importance. These considerations lead to the necessity of understanding how the ECs are reacting during culture, in interaction with scaffolds, and when exposed to shear flow. Efforts need to be made to characterize endothelial cell behavior, especially since endothelial cells commonly undergo morphological changes in vitro. These changes have been observed in the Tissue Engineering Lab and documented in literature ${ }^{13-16}$. Analyzing these changes enhances the legitimacy of the model by ensuring that the endothelial cells retain their endothelial-like phenotype and function throughout culture and sodding.

In order to develop EC-only BVMs, new scaffolds would likely need to be implemented. While several different scaffolds have been proposed and manufactured in the past, use of these scaffolds could not be justified over the standard 75:25 PLGA scaffolds. Current models with PLGA have been working, and no methods have been established to investigate scaffold cytotoxicity, cell adhesion, or cell proliferation between scaffold types. These methods must be developed in order to propose novel scaffolds that can be used for EC only BVMs. Important considerations include the ability to sterilize the scaffold, retention of scaffold geometry, scaffold cytotoxicity, EC 
adhesion at short timepoints, and EC coverage at longer timepoints. The BVM model must be broken down component by component to thoroughly assess the completed model.

\subsection{BVM Characterization}

BVMs must be characterized by the individual components of cells and scaffolds, both separately and together. qPCR and immunostaining are useful techniques to analyze cells. qPCR collects quantitative measurements of gene expression, which indicate changes to specific genetic markers. This technique is used in molecular diagnostics to detect and quantify bacteria, as well as in disease diagnostics to understand how genetic markers change with disease onset and progression ${ }^{17}$. Immunostaining uses antibodies to label proteins in cells, which can be visualized by confocal microscopy to identify protein

presence or intensity ${ }^{11}$. Both techniques can be applied to BVM characterization through analyzing gene expression and protein changes in $\mathrm{ECs}^{11,12,18}$. Gene expression analysis will be extensively covered in Chapter 2 of this thesis.

Methods are also necessary to characterize scaffolds before cells are sodded. Scanning electron microscopy (SEM) focuses an electron beam on a sample and collects the scattered electrons to generate an image. These high-resolution images work well for imaging electrospun scaffolds, where the images can be used to quantify data regarding fiber diameter and porosity. This quantification processing can be done using software like ImageJ or MATLAB ${ }^{19}$.

Once the individual components have been characterized, the interaction between cells and scaffold can be evaluated. The previously identified methods are useful for this 
process. SEM can visualize cell coverage, either in the lumen of scaffolds or on deployed stents. qPCR can quantify cell changes as they interact with different scaffold types, flow conditions, or lengths of time cultured in bioreactors. Immunostaining can be used for similar purposes, characterizing the cell interaction with the scaffold through investigations of adhesion, proliferation, or cell identification.

\subsubsection{Previous Characterization}

Several techniques have been investigated and implemented over the years in the Tissue Engineering Lab to characterize cells in the BVMs. Multiple previous projects have attempted to characterize BVMs through qPCR. Tristin McGuffick evaluated gene expression changes in stented and unstented vessels. PECAM, ICAM, SMA, Ki67, and BAX were chosen as endothelial cell, inflammation, smooth muscle, proliferation, and apoptosis markers, respectively. She found downregulation of PECAM after stent deployment in umbilical BVMs, while coronary BVMs downregulated SMA and upregulated Ki67 and BAX ${ }^{18}$. Shelby Kunz and Gabriella Sugerman used gene expression to characterize ECs cultured in high glucose conditions. Kunz used the markers eNOS, PECAM, and RAGE to characterize and compare HUVECs and diabetic human coronary artery ECs (DHCAECs). DHCAECs had higher expression of RAGE and eNOS compared to HUVECs. HUVECs treated with high glucose media had increased expression of RAGE compared to normal media. These findings set baseline gene expression levels and showed how BVMs using HUVECs differed from those using DHCAECs ${ }^{12}$. Sugerman continued work with high glucose treatments of ECs, using IL-6 and CD36 as markers for oxidative stress. She found that IL-6 expression increased with 
glucose treatment ${ }^{11}$. These three theses struggled to identify a consistent housekeeping gene, making conclusions regarding treatment trends more difficult to confirm.

Imaging techniques have been implemented in many projects, mostly through SEM imaging or immunostaining. SEM images are routinely used to verify a confluent cell lining in the lumen of the BVM. While useful, this technique cannot easily quantify cell presence. Immunostaining techniques have also been used for various purposes. Most common have been nuclear stains to identify and quantify cell presence. Another important project has been to implement staining protocols to distinguish ECs versus SMCs in $\mathrm{BVMs}^{20}$. PECAM has been used as a marker for ECs. Myosin heavy chain, $\alpha-$ smooth muscle actin ( $\alpha \mathrm{SMA})$, and, most recently, calponin have been used to identify SMCs.

\subsubsection{Need for Assessment}

Despite the past work done, there is still need for additional characterization of cells, scaffolds, and BVMs. It has been observed that endothelial cells undergo morphological and genetic changes during culture in vitro ${ }^{21}$. The endothelial lining of the BVMs is, arguably, the most important component of the model. Intravascular devices will theoretically primarily interact with the endothelial lining of the BVMs, and that interaction will provide useful preclinical data. Observations of interest, such as a change in proliferation, migration over the stent, or death, are all observations that have to do with changes in ECs. Therefore, further characterization of ECs specifically is of great importance. 
There are several potential areas where better characterization of endothelial cells would progress our research. The first is to better understand how ECs change at different timepoints in the BVM fabrication process. The first interval is the time between when ECs begin culture in flasks until just before sodding into scaffolds. This information would allow us to track the utility of our cells as they age and identify an optimal passage number to use in BVMs. Depending on how, or if, the cells change, it would also suggest how culture procedures could be changed to optimize EC cell growth. The second interval is the time between when cells are sodded into scaffolds and the resultant BVM is taken down for assessment. There is a need to assess the effect of shear stress on endothelial cells in the BVM model, and to compare how those cells have changed relative to the initial cells that were sodded. A third time interval, when a device is implanted in the BVM, could be included in this section; however, the first two intervals were the focus of this thesis.

The second area for better characterization involves the cell-scaffold interaction. This includes both the cell interaction with the scaffold and the scaffold interaction with the cells. The Tissue Engineering Lab has no current methods to assess these interactions, and without these protocols there is no way to justify or quantify a change from one type of scaffold to another. This must be assessed through initial adhesion data at short timepoints, as well as multi-day time points to address cell proliferation on the scaffold. Scaffold cytotoxicity must also be investigated, especially regarding changes in polymer type or the addition of supplements to the electrospinning solution. 


\subsection{Summary and Aims of the Thesis}

BVMs have proven to be a useful model for testing vascular devices. This utility warrants more exploration into the details of the individual components being used in the model. Before the components can be investigated, methods must be established and verified. These methods will be applied to current problems encountered by the Tissue Engineering Lab to determine the effectiveness of the methods. The overall goal of this thesis was to establish methods that could be used to understand endothelial cell changes in the BVM model. In order to accomplish this goal, two aims were pursued: 1) to characterize ECs alone through changes in gene expression, and 2) to characterize ECscaffold interactions. Each aim is further summarized below.

\subsubsection{Aim 1: Endothelial Cell Characterization through Changes in Gene Expression}

The goal of the first aim of this thesis was to analyze gene expression changes in endothelial cells with a focus on endothelial cell dedifferentiation. The markers explored can also be applied to a broader understanding of cell-cell interactions between ECs. This first aim can be divided into two components relating to changes in HUVEC gene expression. The first component studied the effect of culture time on EC expression, where the treatment groups were defined by the passage number of the cells. The hypothesis was that increasing cell culture duration would decrease endothelial marker gene expression and increase mesenchymal marker gene expression. The second investigation sought to examine the effect of three flow conditions on gene expression. The flow conditions in question were static culture, disturbed flow, and laminar flow. The 
hypothesis here was that static and disturbed flow would decrease endothelial marker expression and increase mesenchymal marker expression, while laminar flow would have the opposite effect on endothelial and mesenchymal markers.

\subsubsection{Aim 2: Characterization of Endothelial Cell and Scaffold Interaction}

The second aim of this thesis sought to establish methods for investigating and quantifying cell-scaffold interactions. This was achieved through designing two assays. The first assay investigated scaffold cytotoxicity through exposure of ECs to scaffold elution products. The second assay was developed to understand cell adhesion on scaffolds at shorter timepoints and cell coverage in BVMs at longer timepoints. Cell apoptosis and dead cell presence were also quantified, respectively, through Caspase and Propidium Iodide activity. These assays were developed and applied to an initial investigation of novel nanofiber scaffolds, with the potential of replacing the current scaffolds used by the Tissue Engineering Lab with the novel scaffolds. 


\section{CHAPTER 2. AIM 1: GENE EXPRESSION CHANGES OF ENDOTHELIAL CELLS IN CULTURE AS A FACTOR OF TIME AND FLOW CONDITION}

\subsection{Introduction}

The first aim of this thesis explored the gene expression changes experienced by endothelial cells in culture as a factor of time and flow condition. In order to understand these changes, several topics must be prefaced, including flow environment, cell phenotype, endothelial-mesenchymal transition, the mechanosensory complex, and qPCR. These topics will provide background and justification for the methods chosen in this aim and will help with explanation of the resulting data.

\subsubsection{Flow Environment}

Endothelial cells are highly reactive to flow conditions of fluid across the cells. Native flow environments within blood vessels are dynamic; vasodilation and constriction, changes in blood pressure and heart rate, and bifurcations all effect blood shear forces across the endothelium. BVMs have consistent and controlled flow, but the forces within BVM are not representative of physiological conditions. Flow within native vessels and BVMs will be described in this section.

\subsubsection{Native Flow Environment}

A baseline set of in vivo flow conditions must be established to compare the BVM model against. The lumen diameter of BVMs range from $3 \mathrm{~mm}$ to $4 \mathrm{~mm}$ depending on the fabrication technique. Therefore, comparable arteries should have similar lumen sizes and previously measured hemodynamic force values for shear stress and cyclic strain. 
Shear stress is the tangential force of blood flowing over the endothelial cells of the tunica intima, and has values ranging from 10 to 70 dynes $/ \mathrm{cm}^{2} 22$. Vasoregulatory mechanisms attempt to maintain a shear stress around 15 dynes $/ \mathrm{cm}^{223}$. Cyclic strain is derived from blood pressure and produces forces that stretch the vessel circumferentially ${ }^{24}$. While shear stress mostly affects the tunica intima, cyclic strain affects all three layers of the vessel ${ }^{22}$. For the purpose of this thesis, shear stress will be the primary focus of hemodynamic force, which is indicated in Figure 5.

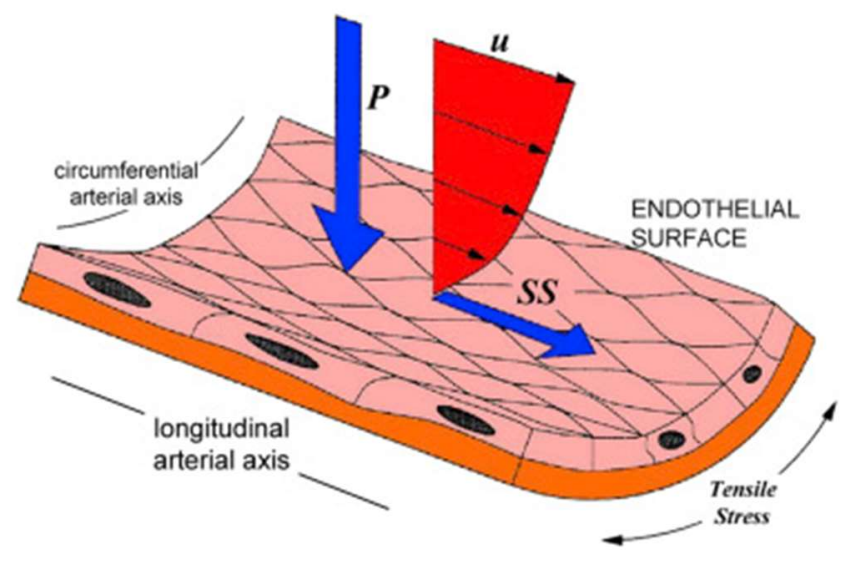

Figure 5: Forces Acting on Blood Vessels. Hemodynamic forces of shear stress (SS), pressure $(\mathrm{P})$, and tensile stress on the endothelium ${ }^{24}$.

One such artery that is a suitable comparison for the BVM model is the left coronary artery, which is on average $4.0 \mathrm{~mm}$ in diameter and has wall shear stress forces between 15 and 40 dynes $/ \mathrm{cm}^{2}$ in areas of undisturbed flow ${ }^{25,26}$. The coronaries are also a common sight of device use, with $91 \%$ of percutaneous artery interventions involving stenting in $2006^{27}$. The carotid artery is also useful for comparing to BVMs. While larger than BVMs at 4.3 to $7.7 \mathrm{~mm}$ in lumen diameter, the mean wall shear stress is lower than that of the left coronary artery at 12 to 15 dynes $/ \mathrm{cm}^{2} 28,29$. The carotids are also a site where stenting is a common treatment ${ }^{30}$. The HUVECs used in the BVMs are sourced 
from umbilical veins, which have an average wall shear stress of 5.2 dynes $/ \mathrm{cm}^{2}$ and a lumen diameter of between 600 and $800 \mu \mathrm{m}^{31,32}$.

Native vessels have areas of laminar and disturbed flow, shown in Figure 6, so wall shear stress is not consistent ${ }^{33}$. Disturbed flow is common near bifurcations and bending in the vessel path where blood flow enters low pressure regions, slows the blood velocity, and causes circulation ${ }^{24}$. These areas, like the one shown in Figure 7, experience less shear stress than under laminar flow, inducing an active phenotype in the ECs that have proinflammatory, pro-adhesive, procoagulant, and pro-proliferative properties ${ }^{34,35}$. Laminar flow and high shear stress, on the other hand, induces a quiescent phenotype that promotes cell alignment and survival while reducing proliferation ${ }^{22,34}$.
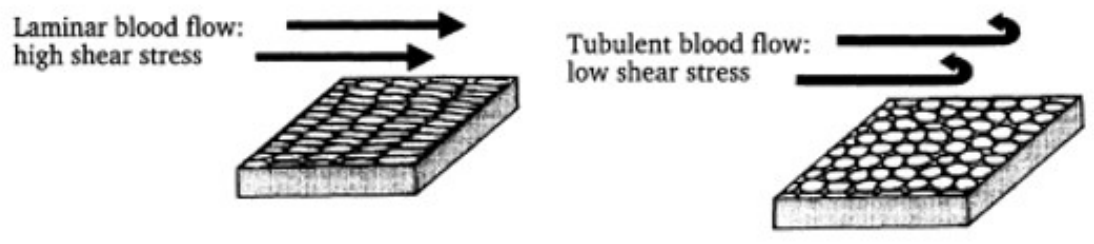

Figure 6: Laminar Flow versus Shear Flow ${ }^{22}$. 


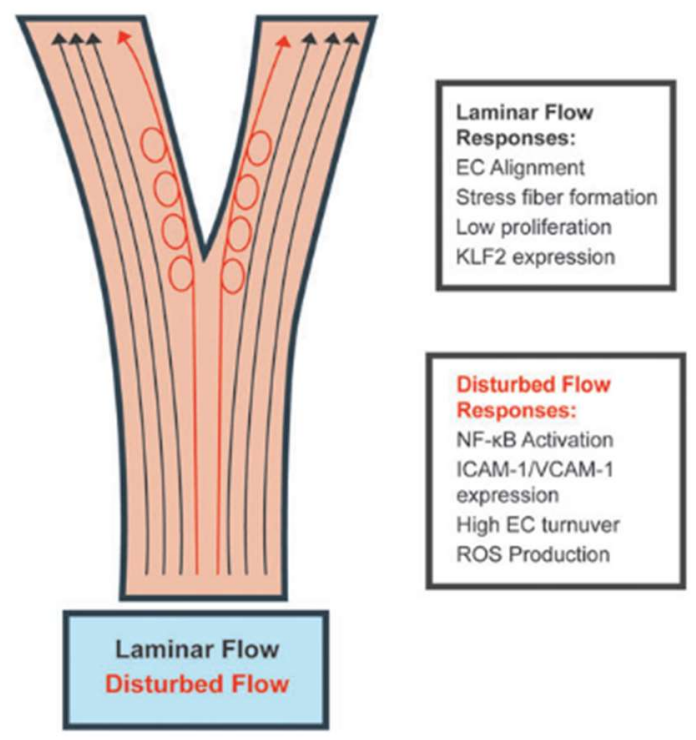

Figure 7: Flow in a Bifurcation. Disturbed flow results at areas of bifurcation, which results in EC activation ${ }^{36}$.

\subsubsection{Previously Determined Flow Conditions in Blood Vessel Mimics}

Previous Biomedical Engineering graduate student Marc Dawson explored

physiological flow conditions in $\mathrm{BVMs}^{37}$. He completed his thesis in 2009 , so almost 10 years have passed since his explorations of BVM flow conditions. Dawson explored two main facets of flow: pressure and wall shear stress (WSS). In vivo changes in pressure only significantly affect the tunica media, which corresponds to changes in the layer of SMCs in BVMs. Therefore, changes in pressure may not significantly affect the EC layer in the BVM, which is the primary concern of this thesis. In his pressure tests, Dawson did determine that the 8-roller pump produced a pressure profile with only small pressure fluctuations at high RPMs. At 100 RPMs, or $10 \mathrm{~mL} / \mathrm{min}$, the 8-roller pump produced pressures of $16 \mathrm{mmHg}$ at maximum and $8 \mathrm{mmHg}$ at minimum. This testing lead to the implementation of an 8-roller peristaltic pump over a 3-roller, so all shear stress measurements were made using the 8-roller pump. 
Dawson determined that his BVM systems had shear stress values of about 0.35 dyne $/ \mathrm{cm}^{2}$, which is significantly lower than physiological shear stress of around 10 dyne $/ \mathrm{cm}^{2}$. Since previous studies determined that EC alignment occurred between 6-10 dyne $/ \mathrm{cm}^{2}$, Dawson attempted to produce WSS values of between $6-8 \mathrm{dyne} / \mathrm{cm}^{2}$. He initially used Dextran and Methylcellulose as additives to cell media to increase the viscosity, and therefore shear stress, in the BVMs. However, Methylcellulose was determined to be too difficult to implement so only Dextran was pursued as an additive. The lowest flow rate used in calculating WSS was $15 \mathrm{~mL} / \mathrm{min}$, or $140 \mathrm{RPMs}$, which produced WSS of 6.04 dyne $/ \mathrm{cm}^{2}$ for a $4 \mathrm{~mm}$ vessel and $14.4 \mathrm{dyne} / \mathrm{cm}^{2}$ for a $3 \mathrm{~mm}$ vessel. To produce this WSS the viscosity of the media would need to be $15.2 \mathrm{cP}$, or almost $30 \%$ Dextran. That percentage of Dextran could negatively affect cell growth, so a lower percentage was implemented in the BVMs. He used 14\% Dextran at $28 \mathrm{~mL} / \mathrm{min}(270$ RPM) to produce a WSS of 6.4 dyne $/ \mathrm{cm}^{2}$. This concentration of Dextran was not found to significantly disrupt cell growth, but the desired cell morphology was not produced.

Several important changes have been made to the model since Dawson's thesis. Scaffolds constructed of ePTFE used to be used in the BVMs, and the standard size of these scaffolds were $4 \mathrm{~mm}$. The current model uses PLGA scaffolds, which have significantly different porosity and cellular interactions, and a standard vessel size of 3 $\mathrm{mm}$. The flow tested by Dawson, especially regarding his exploration of increasing WSS by increasing media viscosity, resulted in higher shear values than in the current model. The lowest flow rate he investigated at standard media viscosity was $10 \mathrm{~mL} / \mathrm{min}$, which corresponds with 100 RPMs on the 8-roller peristaltic pump. The current model uses a maximum flow rate of 90 RPMs. These differences have significant impacts on fluid 
flow, especially regarding shear stress. The use of a $3 \mathrm{~mm}$ vessel instead of $4 \mathrm{~mm}$ could make the implementation of dextran in cell media more feasible since a lower percentage of dextran would produce the same shear stress. However, the lower RPMs of the pump could counteract any difference that the smaller diameter increases the WSS. Exploration of these differences may be necessary in future work, as adjustments to the flow conditions currently used in BVMs may benefit cellular responses like cell adhesion, growth, and phenotype. Nevertheless, this information is just worth noting for this current thesis as background on the model.

\subsubsection{BVM Cell Phenotype}

Phenotype refers to the appearance of a cell as a result of genetic and environmental interactions. In the BVM model, culture conditions and flow conditions contribute to a distinct EC phenotype. Whether this phenotype is similar to a physiologic phenotype has yet to be determined, but this section will elucidate the current understanding of EC phenotype in BVMs and offer a probable explanation for this observed phenotype as background for the methods and experiments in this chapter.

\subsubsection{Immunostaining of HUVECs in the Tissue Engineering Lab}

A previous project in the Tissue Engineering Lab worked to implement immunostaining for the purpose of discerning between ECs and SMCs in BVMs. During the process of developing this protocol, it was noticed that the HUVECs were staining positive for alpha smooth muscle actin ( $\alpha \mathrm{SMA})$, which was the marker that was being used to identify SMCs. This finding is shown in Figure 8. After testing for bleed through 
from other channels and testing controls for the primary and secondary probes, it was concluded that the HUVECs were expressing $\alpha$ SMA, which was hypothesized to potentially be due to the cells undergoing EndMT.

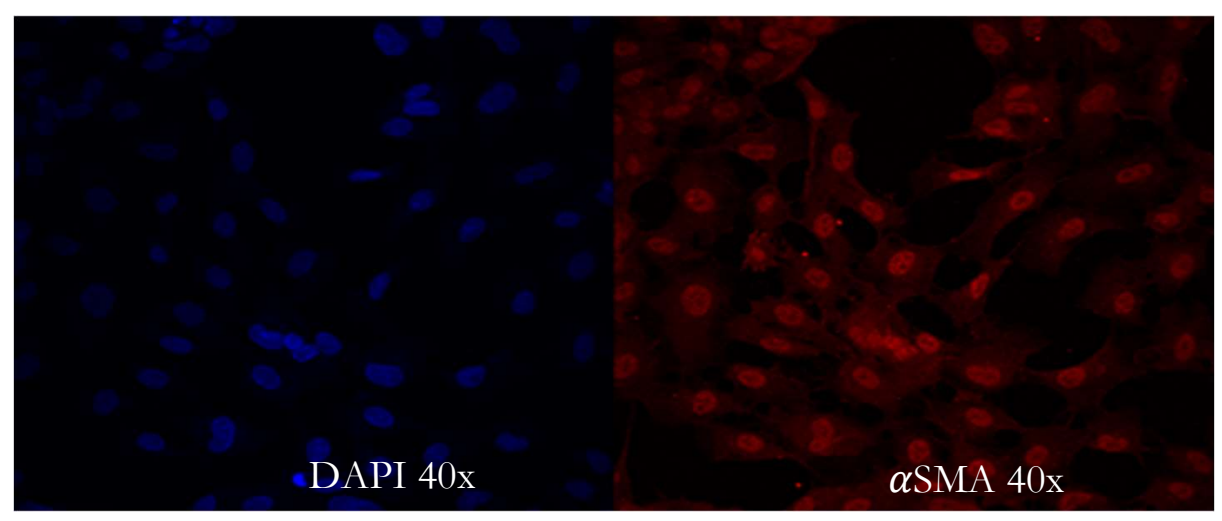

Figure 8: Endothelial Cells Stain for $\alpha$ SMA. HUVECs stained for DAPI, left, and $\alpha \mathrm{SMA}$, right, show expression of $\alpha \mathrm{SMA}$ in HUVEC cytoplasm and nucleus.

\subsubsection{Endothelial-to-Mesenchymal Transition}

Endothelial-to-mesenchymal transition (EndMT) is a process where endothelial cells lose endothelial features and gain fibrotic features ${ }^{13,38}$. During this process, the expression of EC markers, such as VE-Cadherin (VE-cad), platelet endothelial cell adhesion molecule (PECAM), and vascular endothelial growth factor receptor 2 (VEGFR2), decrease while the cells increasingly begin to express fibrotic and smooth muscle markers like $\alpha \mathrm{SMA}$, calponin, and transgelin (SM22 $\alpha)^{21,39-41}$. The mesenchymal phenotype is also characterized by the loss of cell-cell junctions and the gain of migratory abilities $^{42}$. This process may be the reason that HUVECs in the Tissue Engineering Lab express $\alpha$ SMA. Context behind EndMT is necessary to understanding why the phenomenon occurs, and the utility and hinderance of EndMT in vitro and in vivo. The following sections will summarize these topics. 


\subsection{EndMT in Heart Development}

EndMT was first discovered as a mechanism of heart development in utero ${ }^{2,42,43}$. In the early stages of heart development, no valves exist to prevent backflow ${ }^{44}$. Endocardial cushions are thick areas of tissue in the forming heart that eventually develop into the septum, mitral valve, and tricuspid valve ${ }^{45,46}$. During endocardial cushion formation, ECs undergo EndMT and the mesenchymal cells migrate to the endocardial cushions ${ }^{42,45}$. The endocardial cushions elongate to form the septum and valves at different stages of heart formation ${ }^{45}$.

\subsection{EndMT in Disease Progression}

EndMT is involved in various diseases, including fibrosis (cardiac, pulmonary, kidney, liver ${ }^{41,47,48}$, cancer tumor growth ${ }^{16,41,42,47}$, fibrodysplasia ossificans progressiva $(\mathrm{FOP})^{40,47}$, atherosclerosis and coronary artery disease $\mathrm{e}^{2,49-52}$, and cerebral cavernous malformation $^{40,53}$. The involvement of EndMT continues to be discovered in increasing types of diseases.

As previously discussed, blood vessels have areas of laminar and disturbed flow; these areas of disturbed flow are prone to the formation of atherosclerotic lesions ${ }^{2,49,50}$. Specific areas of disturbed flow include carotid bifurcations, the aortic arch, and branching points of the coronary arteries ${ }^{51}$. EndMT-derived fibroblast-like cells have been identified in human atherosclerotic plaque through the co-expression of endothelial and smooth muscle markers ${ }^{49,50}$. This process is caused by TGF- $\beta$ signaling, oxidative stress, and hypoxia. Fibroblast growth factor (FGF) signaling is an inhibitor of EndMT, but disturbed flow inhibits FGF signaling by blocking expression of FGF receptors. 
Hypertension, hyperglycemia, and obesity increase endothelial oxidative stress, making those conditions risk factors of EndMT and atherosclerosis ${ }^{2,49,54}$.

Solid tumors are composed of multiple cell types, including cancer cells, endothelial cells, inflammatory cells, and fibroblasts ${ }^{16,40,47}$. Cancer-associated-fibroblasts (CAFs) are a subpopulation of fibroblasts in tumors and have been identified as promoters of tumor growth and progression ${ }^{41,47}$. Up to $40 \%$ of CAFs in tumors may be derived through EndMT, but the underlying mechanisms for CAF activation and development from EndMT must be further studied ${ }^{42,47}$.

It is still debated as to the extent that EndMT causes fibrosis; other sources of activated fibroblasts arise from local fibroblasts, epithelial-to-mesenchymal transition (EMT), and from the bone marrow ${ }^{15}$. All fibrotic conditions display elevated expression of genes encoding for collagens and ECM and a reduction in ECM degrading enzymes ${ }^{48}$. In three mouse models of kidney fibrosis, $30 \%$ to $50 \%$ of fibroblasts originated from endothelial origin ${ }^{15}$. A mouse model for cardiac fibrosis demonstrated that $27 \%$ to $35 \%$ of fibroblasts in the fibrosis tissue originated from EndMT $^{55}$. The contribution of EndMT in human tissue has less robust evidence. However, a study analyzing myocardial tissue from patients with cardiac fibrosis showed that about $17 \%$ of fibroblasts in the fibrotic myocardium were derived from EndMT ${ }^{48}$. This suggests that dedifferentiated endothelial cells are a significant component of atherosclerotic plaque in humans.

\subsection{EndMT in Tissue Regeneration}

While EndMT is highly involved in disease progressions, it also has the potential to assist in tissue regeneration in vitro and in vivo. Since EndMT differentiates ECs into a 
mesenchymal stem cell-like phenotype, those mesenchymal cells can be further differentiated into other tissue types, as shown in Figure $9^{53,56}$. One potential application for EndMT in tissue regeneration is for the treatment of osteoarthritis and temporomandibular joint disorder (TMJD) ${ }^{53}$. Chondrogenesis is the process by which cartilage is formed, where chondrogenic mesenchymal cells proliferate into precartilaginous nodules before differentiation into chondrocytes producing cartilage $\mathrm{ECM}^{57}$. Cartilage grafts could be produced using EndMT derived chondrocytes. A similar concept was shown with osteogenesis in mice, where endothelial cells labeled with fluorescent quantum dots and treated with TGF $\beta 2$ were seeded onto scaffolds, implanted, and locally treated with differentiation media. The scaffolds were explanted and stained for bone, in which it was found that the scaffolds stained for bone in the same region that the quantum dot fluorescence occurred ${ }^{58}$. This displays the utility of endothelial cells and the ability of the cells to readily differentiate in proper conditions. The conditions of static culture and of BMVs may be appropriate conditions to cause EndMT.

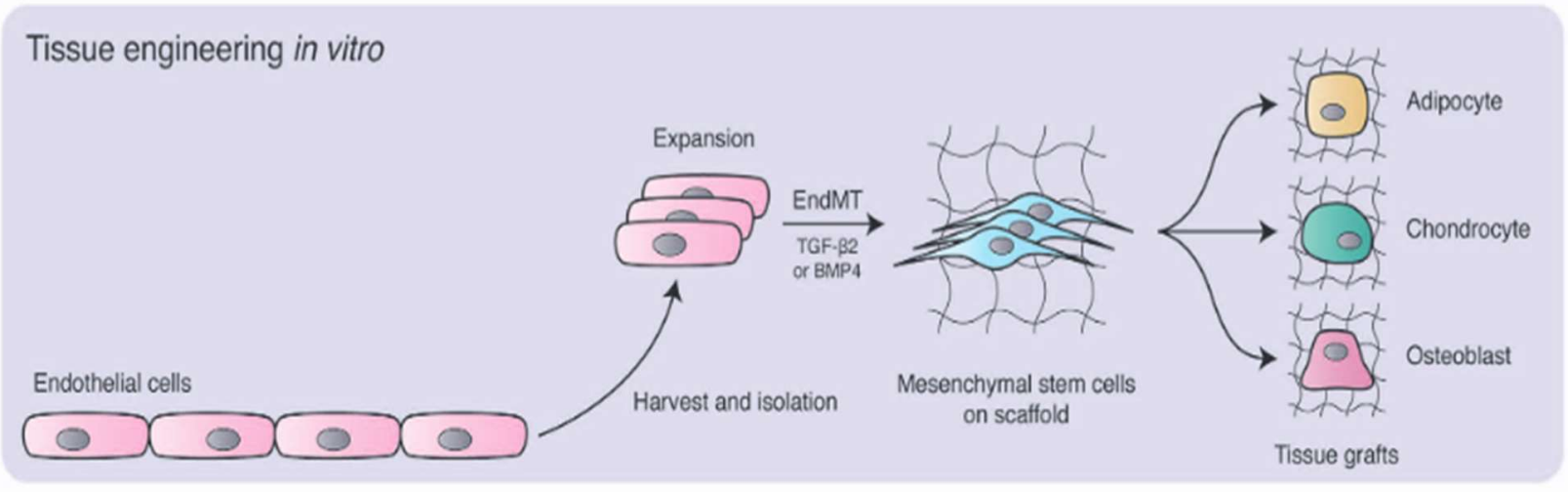

Figure 9: EndMT in Tissue Engineering. Potential use of EndMT in tissue engineering through dedifferentiating ECs into multiple other cell types ${ }^{56}$. 


\subsubsection{EndMT Activation Pathways}

The progression of EndMT in ECs is most easily explained through the pathways of the different inducers, which includes transforming growth factor beta (TGF- $\beta$ ), the pro-inflammatory cytokines interleukin-1 beta (IL1- $\beta$ ) and tumor necrosis factor-alpha $(\mathrm{TNF} \alpha)$, transcription factor NF-kB, and oxidative stress ${ }^{38}$. Each of these inducers will be introduced below.

\subsection{Transforming Growth Factor Beta}

The most well studied EndMT inducer is TGF- $\beta$, a growth factor that has roles in cell proliferation, differentiation, apoptosis, and migration. It has three isoforms (TGF$\beta 1$, TGF- $\beta 2$, TGF- $\beta 3$ ) that bind to TGF- $\beta$ transmembrane receptor type II (TGF $\beta-$ RII). This phosphorylates and activates one of two TGF- $\beta$ type I receptors, either activin receptor-like kinase 5 (ALK5) or ALK1. ALK5 phosphorylation activates SMAD2/3, while ALK1 activates SMAD1/5. Both SMAD2/3 and SMAD1/5 form complexes with co-SMAD (SMAD4) and move into the nucleus. High levels of TGF- $\beta$ will signal mainly through ALK5, while low levels of TGF- $\beta$ will signal through ALK5 and ALK1. Endoglin (END) acts as an auxiliary receptor that inhibits ALK5 signaling and promotes ALK1 signaling ${ }^{13,38,48,59}$.

This point in the signaling is important, as ALK1 and ALK5 cause opposite effects. ALK1 signaling promotes EC proliferation, migration, and organization; ALK5 signaling inhibits EC proliferation, migration, organization, and continues the EndMT pathway. SMAD2/3 induces expression of Snail1, Slug (Snail2), Twist1, and GATA4. These are the transcriptional regulators of EndMT in the nucleus of ECs. GATA4 
regulates Twistl expression, which induces Snail pathway activation in $\mathrm{ECs}^{2}$. The Snail pathways downregulate VE-cadherin, an important protein in cell-cell interactions and an

indicator of EndMT progression ${ }^{60-62}$. However, the Snail pathway alone is insufficient in inducing EndMT: GSK-3 $\beta$, the inhibitor of Snail, needs to be inhibited through phosphorylation for EndMT to occur ${ }^{62}$.

TGF- $\beta$ also activates mitogen-activated protein kinase (MAPK) pathways that activate the Snail pathway downstream. This includes extracellular signal-regulated kinase (ERK)1/2, p38 MAPK, and c-Jun-N-terminal kinase (JNK) [50]. The TGF- $\beta$ provides a pathway for positive feedback that sustains EndMT progression. TGF- $\beta$ activates TGF- $\beta$-activated kinase 1 (TAK1), which activates p38 MAPK expression. p38 MAPK increases the expression of TGF- $\beta$, creating a positive feedback loop for EndMT ${ }^{2}$. Therefore, EndMT can occur without constant stimulation by TGF- $\beta$, making this pathway a possible source of EndMT in vitro.

\subsection{Notch Signaling}

While TGF- $\beta$ is considered the main inducer of EndMT, several other pathways contribute by aiding the TGF- $\beta$ pathway ${ }^{62}$. Notch can activate Twist 1 expression, contributing to the activation of the Snail pathway ${ }^{2}$. Notch also upregulated mesenchymal genes by recruiting $\mathrm{Smad} 3$ to $\mathrm{Smad}$ binding $\operatorname{sites}^{48}$. Snail can also be directly upregulated by Notch signaling; however, this role of is more robust during development of the cardiac cushion $^{60}$. 


\subsection{Wnt/ $\beta$-catenin Signaling}

$\mathrm{Wnt} / \beta$-catenin signaling is necessary for heart valve development in utero, but also plays a role in tissue fibrosis ${ }^{48}$. It contributes indirectly to EndMT by stabilizing Snail, while contributing directly by inducing expression of transgelin (SM22- $\alpha)^{2}$. The Wnt pathway also plays a role in EndMT caused by hyperglycemic conditions ${ }^{63}$.

\subsection{Pro-inflammatory Cytokines}

Several cytokines associated with the inflammatory response have been shown to induce EndMT. Interleukin 1 beta (IL-1 $\beta$ ), interleukin 6 (IL-6), and tumor necrosis factor alpha (TNF- $\alpha$ ) activate NF- $\kappa$ B through the Akt pathway ${ }^{38,64}$. NF- $\kappa$ B translocates to the nucleus where it affects gene expression. It is not apparent exactly how NF- $\kappa \mathrm{B}$ changes gene expression, but NF- $\kappa \mathrm{B}$ is required for EndMT to occur. When the NF- $\kappa \mathrm{B}$ inhibitor $\mathrm{I} \kappa \mathrm{B} \alpha$ remained active in HUVECs, mesenchymal markers were not expressed ${ }^{65}$.

Interestingly, the treatment of these cytokines themselves does not seem to create enough stimulus to cause EndMT. IL-1 $\beta$ treated ECs only increased expression of SM22$\alpha$ when they were also treated with TGF $\beta$, suggesting that IL-1 $\beta$ alone does not cause EndMT $^{66}$. These cytokines also have varying strengths of inducing EndMT, with TNF- $\alpha$ being a more potent inducer than IL- $6^{64}$.

\subsection{Oxidative Stress}

Reactive oxygen species (ROS) include hydroxyl radicals, superoxide radicals, hydrogen peroxide, and peroxynitrite. Most ROS production is the result of NADPH oxidase system, specifically NOX4, with which the production of ROS activates NF- $\kappa \mathrm{B}$ 
and leads to EndMT ${ }^{38,55}$. ROS production also activates p38, which leads to the production of TGF- $\beta$ and continues the TGF- $\beta$ signaling of EndMT ${ }^{38}$. In normal cell functioning, TGF- $\beta$ stimulates the generation of ROS, so the TGF- $\beta$ pathway of inducing EndMT will inherently cause ROS production, adding another source of EndMT stimulus $^{67}$.

\subsection{Disturbed Flow}

Areas of disturbed flow, where it was previously established that EndMT occurs, are prone to the activation of the pathways that cause EndMT. Disturbed flow activates inflammatory signaling, ROS production, and TGF- $\beta^{2}$. Areas of disturbed flow are also prone to high blood pressure and stiffening of the vascular walls, which causes high cyclic strain. Cyclic strain is the stretch force circumferentially experienced by ECs. This strain further activates TGF- $\beta$, while additionally activating Wnt/ $\beta$-catenin signaling. While BVM should experience low levels of circumferential strain, it is possible that the flow through BVMs results in inflammatory pathway activation.

\subsubsection{Inhibitors of EndMT}

Although many factors lead to EndMT onset, there are treatments to inhibit this transition, which may be useful as cell treatments during culture. Common media additives like FGF2 induce the expression of microRNA-20a (miR-20a). MiR-20a blocks ALK5 (TGF $\beta$ RI) and TGF $\beta$ RII, inhibiting TGF $\beta$ activation of Smad2 ${ }^{68}$. FGF also increases the expression of VEGF in $\mathrm{ECs}^{54}$; the combination of reduced TGF $\beta$ activity 
and increased VEGF expression results in reduction of EndMT. Other proteins that, when added to cell media, reduce EndMT include VEGF-A and BMP7 ${ }^{53}$.

Several drugs reduce the effects of EndMT, which are briefly outlined in table I. However, some have only proven efficacy in animal models, such as Relaxin.

\section{Table 1: Drugs that Inhibit or Reduce EndMT ${ }^{53}$.}

\begin{tabular}{l|l}
\hline Drug & Effect \\
\hline Linagliptin & DPP-4 inhibitor, blocks TGF $\beta 2$ \\
Rapamycin & Blocks mTOR signaling \\
Relaxin & Disrupts Notch signaling \\
Macitentan & Endothelin-1 receptor inhibitor \\
Kallistatin & MMP inhibitor \\
Spironolactone & Aldosterone receptor blocker, inhibit Notch1 expression \\
Scutellarin & Reduces Notch1 expression \\
Losartan & Inhibits angiotensin II type 1 receptor \\
Cinacalcet & Suppresses hormone levels \\
Hydrocortisone & Enhances EC adhesion
\end{tabular}

Alterations in mechanical stimuli can also reduce EndMT. Opposite to how disturbed flow can induce dedifferentiation, laminar shear stress can protect endothelial cells from EndMT. The activation of Kruppel-like factor (KLF) 2 and 4 by extracellular signal-related kinase 5 (Erk5) are responsible; Erk5 is continuously activated by laminar shear stress, and KLF2/4 are transcription factors activated downstream that have a wide range of effects, but mainly target the NF-kB and TGF $\beta$ pathways ${ }^{69,70}$. KLF2/4 directly inhibits NF-kB activation while suppressing activator protein 1 (AP-1) and Smad2 
nuclear translocation, both important proteins in the TGF $\beta$ pathway ${ }^{70,71}$. Furthermore, KLF4 binds to and activates the VE-cadherin promoter, leading to the stabilization of adherens junctions ${ }^{72}$.

The investigation of laminar shear stress offers a plausible and logical starting point to impacting and potentially reducing EndMT in the BVM model. These alterations would not require media supplements or drugs which may be expensive, difficult to obtain, and whose effect may have unanticipated consequences such as toxicity or decreased cell viability. In order to determine if any treatments affect EndMT, gene markers must be identified that correlate with EndMT progression as a result of flow changes.

\subsubsection{The Mechanosensory Complex}

Mechanical connections between endothelial cells are generally known as adherens junctions, which in ECs are important for monolayer integrity, contact inhibition of growth, and apoptosis ${ }^{73}$. One type of adherens junction specific to ECs, known as the mechanosensory complex, is composed of PECAM, VE-cadherin, and VEGFR2. This set of proteins is commonly used to identify and quantify EndMT ${ }^{14,21,49,50,64,69,74,75}$, which may also be applicable for use in characterizing EndMT in HUVECs used for BVMs. The mechanosensory complex is not the only mechanotransducer that causes cellular changes after exposure to flow, as shown in Figure 10. G-proteins, primary cilia, integrins, intermediate filaments, glycocalyx, caveolae, ion channels, and the actin cytoskeleton all have proposed roles in flow transduction $^{36,76}$. However, the mechanosensory complex is primarily responsible for 
maintaining the EC monolayer, and its functions have been well researched in relation to EndMT. This section will discuss the proteins in this complex, how they interact, and how they are stimulated in response to different flow conditions.

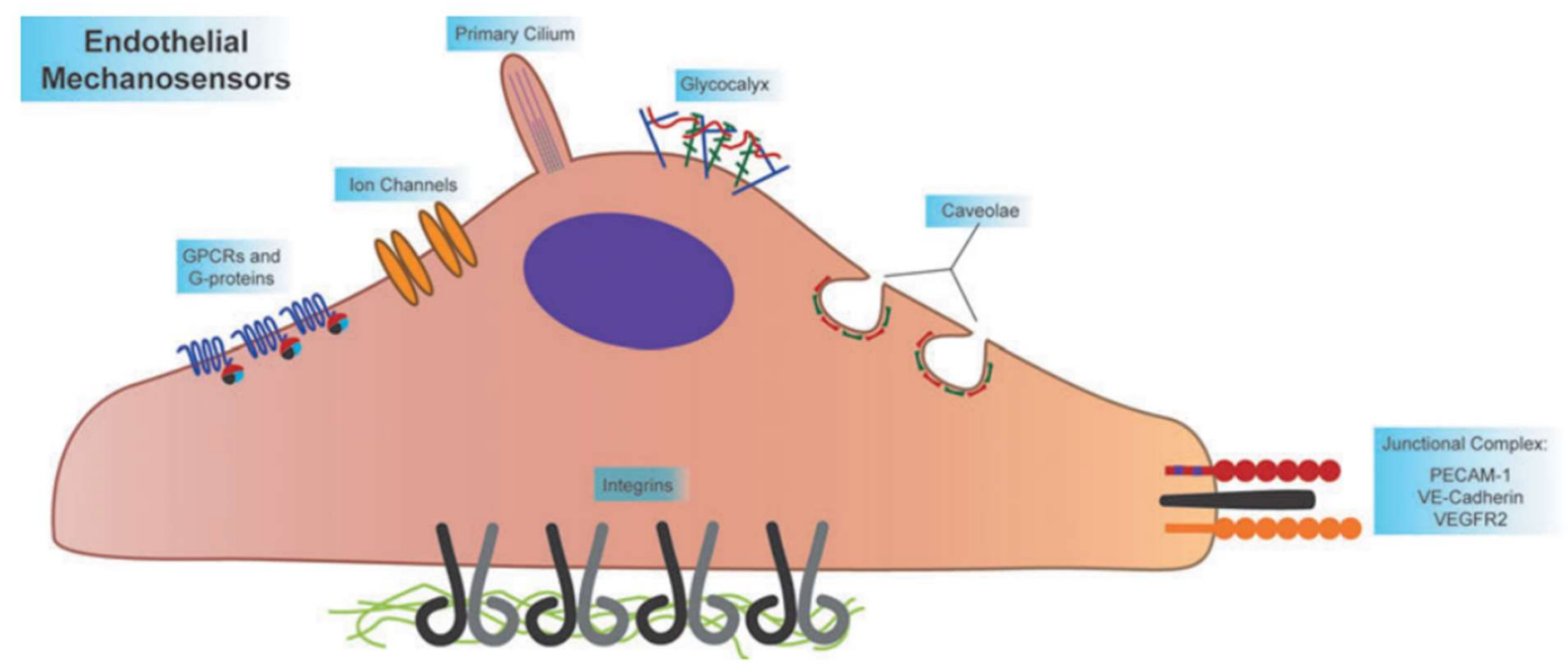

Figure 10: Types of Endothelial Mechanosensors. A depiction of the many mechanotransducers present on endothelial cells ${ }^{36}$.

\subsubsection{Platelet Endothelial Cell Adhesion Molecule 1 (PECAM-1)}

PECAM-1 primarily functions as a mechanosensor and transmitter of mechanical force from shear stress to the cytoskeleton ${ }^{70,73,77}$. Shear stress induces rapid phosphorylation of PECAM and eventual downstream phosphorylation and activation of VEGFR2, making it the principal initiator of the mechanosensory complex when exposed to stresses ${ }^{73,77}$. The expression of PECAM-1, as well as the phosphorylation of PECAM, is highly dependent on cell confluence. $100 \%$ confluent HUVEC cultures showed significantly more PECAM-1 and phosphorylated PECAM-1 expression than 50\% confluent cultures $^{78}$. The Cal Poly Tissue Engineering Lab has verified through 
immunostaining that PECAM-1 expression is reduced in areas where cell confluency is reduced, as cell-cell contact is necessary for PECAM-1 expression ${ }^{20}$. Furthermore, for confluent HUVEC cultures, less phosphorylated PECAM-1 was expressed after exposure to disturbed flow versus laminar flow ${ }^{78}$. This is an important consideration, as PECAM-1 phosphorylation is necessary for mechanosensory complex function.

Several studies have investigated the role of PECAM under direct exposure of mechanical stimuli. Ferromagnetic beads coated with PECAM-1 antibody were used to stimulate the protein with direct mechanical force. After 10 minutes of magnetic exposure, Western blots showed the phosphorylation of both PECAM-1 and ERK $2^{79}$. As previously discussed, ERK 2 is implicated in the activation of Snail, a major contributor of EndMT, and this activation of ERK 2 was verified in ECs exposed to shear stress. Fluorescence resonance energy transfer (FRET) based tension sensors were designed to quantify changes in direct force across PECAM-1 during shear stress exposure ${ }^{80}$. Shear stress on bovine aortic endothelial cells (BAECs) resulted in an increase in tension on PECAM-1 and an association between PECAM-1 and vimentin, an intermediate filament ${ }^{81}$. In the same study, the role of vimentin was explored with a vimentin knockdown, which resulted in blocking the tension increase on PECAM-1. It was concluded that vimentin transmits the mechanical force from flow shear stress to PECAM-1 in order to initiate downstream effects ${ }^{80}$.

Not all literature agrees on the role of PECAM-1 during EC exposure to shear stress, as some studies implicate it in inducing atherosclerosis instead of protecting from or reducing atherosclerosis ${ }^{82}$. PECAM knockout mice showed reduced atherosclerotic lesions in the aortic arch, as well as a decrease in NF- $\kappa \mathrm{B}^{83}$. This may indicate that 
PECAM-1 is associated with NF- $\kappa$ B-induced EndMT and atherosclerosis. It may also indicate that PECAM-1 knockout simply dampens general inflammatory signaling in $\mathrm{ECs}^{36}$. This is most likely the case: since ENOS is deregulated in the absence of PECAM1, PECAM knockout mice have high NO production which suppresses inflammation ${ }^{54}$. Based on this information, the assumed role of PECAM-1 as atheroprotective remains valid; regardless, the necessity of PECAM for transmitting fluid forces and retaining the function of the mechanosensory complex makes it a marker of interest for this aim.

\subsubsection{Vascular Endothelial Cadherin (VE-Cad)}

While the application of force directly to PECAM-1 triggers the same responses as flow shear stress, these effects are not seen when force is applied to VE-cad ${ }^{76,80}$. This demonstrates the functional difference between the two proteins; VE-cad acts as an adaptor molecule that links the mechanical force transduced by PECAM-1 to VEGFR $2^{70,73,76,84}$. This is achieved through the formation of complexes that bind to the actin cytoskeleton that then transfer signals within $\mathrm{ECs}^{70,77,85}$. These complexes are formed with alpha and beta catenin, plakoglobin, vinculin, and cingulin ${ }^{77}$. VE-cad binds directly to $\beta$-catenin or plakoglobin, which then binds the actin binding protein $\alpha$ catenin $^{86}$.

VE-cad is the major determinant of EC contact integrity, so remodeling of VEcad is important to adapt to changes in stimulus ${ }^{77,86}$. During angiogenesis the interactions formed between VE-cad and neighboring cells, as well as the interactions between VEcad and cytoplasmic proteins, must be broken to allow cell migration and for new cellcell adhesions to form ${ }^{87}$. VE-cad interactions are also disrupted with increased matrix 
stiffness due to changes in cytoskeletal mechanics, resulting in increased EC monolayer permeability ${ }^{73}$. Arterial fibrosis associated with EndMT results in positive feedback where increasing matrix stiffness disrupts EC monolayers and encourages uncontrolled cell migration. This relaxation of the EC monolayer was demonstrated with FRET-based tension sensors which saw a rapid $25 \%$ decrease in tension across VE-cad with the onset of flow ${ }^{80}$.

VE-cad plays other important role in response to flow in vitro. VE-cad depleted ECs aligned at lower rates than depleted cells transfected with VE-cad after exposure to 12 dynes $/ \mathrm{cm}^{\wedge} 2$ of shear stress for 18 hours $^{88}$. VE-cad expression does not change in VEcad depleted cells exposed to static or shear flow, but expression doubled in VE-cad positive cells exposed to shear stress versus a static condition ${ }^{14}$. Therefore, VE-cad expression is necessary for HUVECs used in BVMs to align in the direction of flow.

VE-cad also has an important relationship with KLF4, which was previously discussed as an inhibitor of EndMT. VE-cad is transcriptionally regulated by KLF4, in which KLF4 binds the promoter region of VE-cad and induces VE-cad transcription ${ }^{72}$. This promotes VE-cad interactions to sustain the EC monolayer, helping to reduce cell migration and EndMT. VE-cad may be a useful marker to study in HUVECs intended for BVMs, as high expression of VE-cad would suggest EndMT inhibition.

\subsubsection{Vascular Endothelial Growth Factor Receptor 2 (VEGFR2)}

VEGFR2 is the component of the mechanosensory complex that activates biochemical signaling and is necessary for EC migration, proliferation, angiogenesis, and regulation of cell death ${ }^{73,85}$. VEGFR2 is the main RTK that mediates VEGF signaling, 
and thus will be focused on even though VEGFR3 has recently been recognized as a member of the mechanosensory complex ${ }^{73}$. Binding of VEGF to VEGFR2 initiates activation of PI3K and MEKK3, causing downstream activation of Akt and MEK5/Erk $5^{70}$. Erk5 is an inhibitor of EndMT and activates KLF2/4 which promotes VEcad transcription, demonstrating only one of many interactions between VE-cad and VEGFR2. The role of VEGFR2 is highly dependent on its interaction with VE-cad and should therefore be discussed together.

\subsubsection{VE-Cadherin Regulation of VEGFR2}

VE-cad alters cell proliferation and apoptosis through modulating VEGFR2 function. Cell confluence is one critical component on which VE-cad acts to inhibit VEGFR2 function in vitro. Cadherins in general, including VE-cad, transfer negative intracellular signals upon the establishment of cell-cell contact such as when confluence is reached. This results in a decreased response to growth factors like VEGF. Lampugnani et al. investigated the relationship between confluent or sparse VE-cad-null and VE-cad-positive cells stimulated with or without VEGF ${ }^{85}$. VE-cad-null cells continued growth into over-confluence, while VE-cad-positive cells arrested growth at confluence, demonstrating the growth restricting role of VE-cad. VE-cad-null cells were responsive to VEGF regardless of confluence, while VE-cad-positive cells were only responsive in the sparse condition. The lack of response in the VE-cad-positive confluent condition was associated with a reduction of VEGFR2 phosphorylation. The same trend was verified in HUVECs, where an upregulation of VEGFR2 phosphorylation in VEGF 
occurred in sparsely distributed cells. The role of cell density on expression will be important to control during experiments studying VE-cad and VEGFR2.

That study, as well as others, ultimately concluded these interactions were a result of VE-cad forming a complex with VEGFR2 and density-enhanced receptor-like tyrosine phosphatase (DEP-1) $)^{14,85,88}$. In sparse ECs in vitro, VEGF binds VEGFR2 to activate the Erk1/2 pathway. Erk1/2 phosphorylates VE-cad, which causes the detachment of $\beta$ catenin from VE-cad. $\beta$-catenin translocates to the nucleus where it initiates transcription that results in cell proliferation. In confluent ECs, DEP-1 congregates at cell-cell junctions and inhibits Erk1/2 phosphorylation of VEGFR2, blocking $\beta$-catenin detachment and nuclear translocation ${ }^{89,90}$. This mechanism is depicted in Figure 11.

SPARSELY SEEDED ENDOTHELIAL CELLS

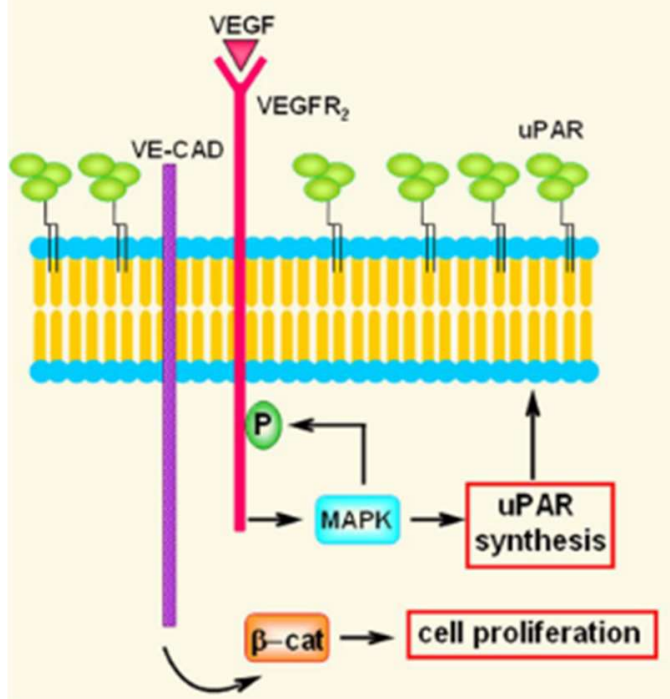

CONFLUENT ENDOTHELIAL CELLS

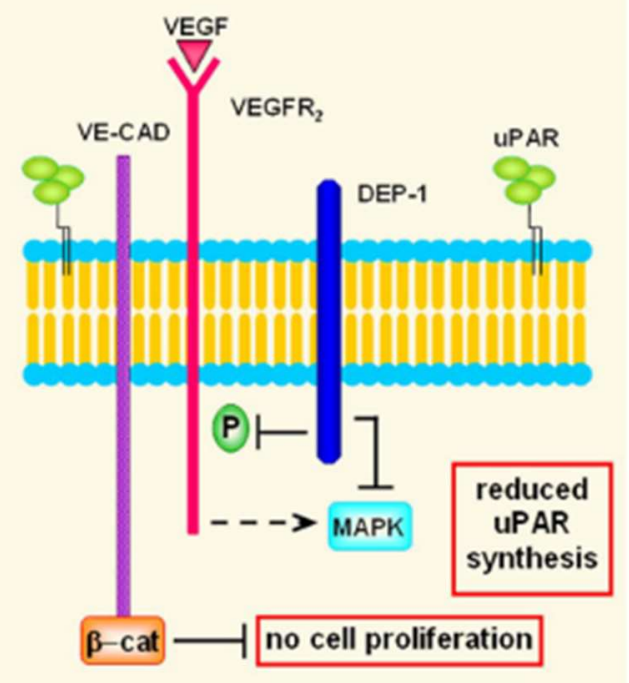

Figure 11: Mechanisms of VE-Cad and VEGFR2 on Cell Seeding Density ${ }^{89}$.

While those interactions are important for understanding the mechanosensory complex in quiescent EC monolayers in vivo and in vitro, it lacks the context of how these interactions fare with alternative, non-physiologic flow conditions such as in 
BVMs. Parallel plate flow models showed that, upon exposure to shear stress, VEGFR2 expression increased and VE-cad promoted VEGFR2 activation instead of inhibiting it $^{91,92} \cdot \beta$-catenin phosphorylation increased in HUVECs exposed to shear stress, indicating that cells were being induced into a proliferative state ${ }^{93}$. The same study found that fluid shear stress causes the dissociation of VE-cad and $\beta$-catenin. While the mechanism isn't entirely elucidated, shear stress ultimately contributes to EC survival by VEGFR $2^{94}$. Interestingly, the type of laminar flow does not affect cell survival in vitro. A TUNEL assay of cells exposed to static media, steady laminar flow, pulsatile laminar flow, and to-and-fro laminar flow showed no difference in percent of apoptotic cells ${ }^{95}$. The length of time in which cells are exposed to these conditions plays a key role in VEcad and VEGFR2 activation, and this duration is key for the induction of EndMT.

\subsubsection{Mechanosensory Complex and EndMT}

The important roles of the mechanosensory complex in maintaining EC monolayers and in flow signaling is undisputed, making it an ideal candidate for understanding and quantifying EndMT in ECs in culture or in BVMs. For this, and to better understand the second component of Aim 1, the focus must shift back to the effects of laminar versus disturbed flow on ECs and how the mechanosensory complex changes. The general difference between these two flow conditions that should be emphasized are as follows: ECs adapt to flow under laminar flow so that EndMT events are downregulated after the initial stimulation, while these events are sustained in disturbed flow $^{14}$. An important example of this involves the activation of Erk1/2 by both PECAM and VEGFR2. Erk1/2 activation stimulates the TGF $\beta$ pathway, activates Snail, and 
eventually leads to EndMT. However, this activation comes with the same caveat, where type of flow is the major consideration in adaptive expression versus sustained expression. While expression of Erk $1 / 2$ is sustained over time, the phosphorylated form is not sustained in laminar flow ${ }^{50}$.

The general characteristics of EndMT, identified by expression of mechanosensory complex markers, is the downregulation of PECAM-1, VE-cad, and VEGFR2. Therefore, if EndMT was occurring during HUVEC culture or BVM creation, all three of those markers would be downregulated. In the case of partial EndMT or partial laminar flow, the expression profile of the mechanosensory complex would not be as straightforward. While stimulation of the mechanosensory complex changes expression of the endothelial markers of EndMT, it does not capture fibrotic changes to the endothelial cells. These changes are evaluated through upregulation of mesenchymal markers.

\subsubsection{Mesenchymal Markers}

EC markers indicate the extent of EndMT away from endothelial characteristics, but mesenchymal markers (also referred to as smooth muscle or fibroblast characteristics in the literature) indicate the extent of EndMT towards the fibrotic state. Common markers include $\alpha$-smooth muscle actin ( $\alpha$ SMA), transgelin (SM22 $\alpha$ ), fibroblast specific protein 1 (FSP-1), calponin, and smooth muscle-myosin heavy chain 2 (SMMHC2 $)^{21,49,50,62,64,69,74,75}$. These markers will generally be associated with cells double positive for EC and mesenchymal/SMC markers, which identify intermediate stages of EndMT. 
Smooth muscle cell differentiation can give insight into mesenchymal marker onset in EndMT. Five molecular markers are commonly used to assess SMC differentiation, which are also markers used to assess EndMT. These include $\alpha$ SMA, transgelin, calponin, caldesmon, and myosin heavy chain (MHC), where $\alpha \mathrm{SMA}$ and transgelin are early markers of SMCs and calponin, caldesmon, and MHC are intermediate to late markers ${ }^{96}$. The early expression of $\alpha \mathrm{SMA}$ and its abundance in differentiated SMCs makes $\alpha$ SMA the most widely used marker for EndMT identification ${ }^{74}$. Therefore, $\alpha \mathrm{SMA}$ is a candidate to include in EndMT evaluation for this aim, especially because $\alpha$ SMA expression has already been observed in HUVECs used in BVMs.

The pathways of EndMT previously discussed stimulate the onset of these mesenchymal markers, especially through the TGF $\beta$ pathway. TGF $\beta$ stimulates the expression of many genes associated with fibrosis, including varieties of collagen and fibronectin, through Smad2/3 and p38 MAPK activation. This pathway also induces collagen cross-linking, which increases stability, and stimulates the synthesis of protease inhibitors, increasing ECM production ${ }^{62}$. In vitro analysis reveals the onset of mesenchymal markers through TGF $\beta$ stimulation. HUVECs in static media stimulated with TGF $\beta$ were found to increase expression of calponin and transgelin, while the same cells in laminar flow did not increase expression of mesenchymal markers ${ }^{50}$. This indicates that EndMT can be induced without flow induction. Erk5, which protects EC monolayers from EndMT, was then silenced and resulted in increased calponin and transgelin expression regardless of the flow condition with TGF $\beta$ stimulation. A different study used orbital shakers without TGF $\beta$ stimulation to induce disturbed flow. They 
found that HUVECs in this these conditions expressed Snail, Slug, and $\alpha$ SMA, markers which indicate that disturbed flow alone was enough to activate EndMT pathways ${ }^{69}$.

Mesenchymal markers are also found in vivo in EC monolayers. ECs positive for $\alpha$ SMA have been found in the aorta of rats, mice, and humans; these cells were determined to be of EC origin based on their co-expression of VE-cad with $\alpha \mathrm{SMA}^{74}$. While these cells were sparsely distributed, the presence of these cells displays EndMT progression in an atheroprone region. Mesenchymal markers are also abundant in atherosclerotic plaques, where EndMT contributes to cell migration and transdifferentiation in these plaques. Atherosclerotic lesions on a porcine bifurcation expressed $\alpha \mathrm{SMA}$, transgelin, and calponin; similarly, human atherosclerotic plaque was shown to co-expresses PECAM-1 and $\alpha \mathrm{SMA}^{13}$.

\subsubsection{Using Endothelial and Mesenchymal Markers to Determine EndMT Progression}

The Tissue Engineering Lab should be concerned with EndMT at all stages of BVM fabrication. EndMT can result from both static culture and disturbed flow. Therefore, the first consideration for this aim was whether the sodded ECs are sustaining an EC phenotype after their time in culture, defined by a cobblestone appearance and the expression of endothelial markers without mesenchymal markers. It has previously been noted that ECs cultured in the Tissue Engineering Lab develop a spindle-like phenotype over time in culture. This consideration has been verified in literature. Freshly isolated adult bovine ECs positive for VE-cad and PECAM-1 displayed EndMT over time in static culture ${ }^{21}$. At day one of culture, cells displayed a cobblestone appearance and were 
negative for SMC marker expression. By the second day of culture, cell-cell contact was disrupted and $\alpha$ SMA was lightly expressed. By day 5 ECs acquired an elongated morphology, increased $\alpha$ SMA expression, and no longer expressed VE-cad. Flow models have also displayed EndMT onset. In a bifurcation model of flow, in which areas downstream of the bifurcation were characterized by disturbed flow, shown in Figure 12, VE-cad expression decreased while transgelin expression increased ${ }^{49}$. Collagen I was also prevalent in the area of disturbed flow, which is the most prevalent collagen type in atherosclerotic plaques. The onset and co-expression of EC and mesenchymal markers allow for EndMT assessment in vitro. However, to understand the progression of EndMT, changes in mesenchymal and endothelial markers must be quantified.

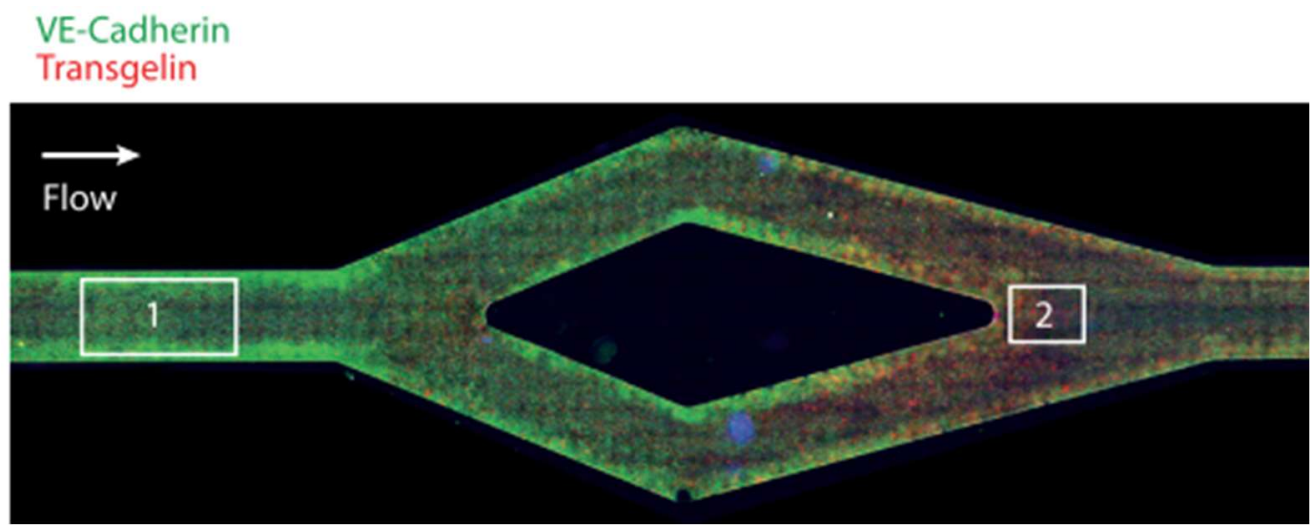

Figure 12: VE-cad and Transgelin Expression in a Simple Flow Model. (1) VE-cad is expressed upstream. (2) Upon turbulent flow induction transgelin expression is present ${ }^{50}$.

\subsubsection{RT-qPCR Background}

Studies of gene expression have previously been implemented in the Tissue Engineering $\mathrm{Lab}^{11,18}$, and the technique of reverse transcription quantitative polymerase chain reaction (RT-qPCR) has been used throughout literature to identify changes in 
EndMT $^{49,50,59,69,97-99}$. For the characterization of ECs, this technique is a powerful and efficient method of determining alterations in genes that are associated with a mesenchymal phenotype over an endothelial phenotype. This technique quantifies the amount of mRNA present in a cell with the assumption that these mRNA will become proteins and effect cell function and behavior. However, total RNA, not specifically mRNA, is often used; total RNA involves fewer purification steps, a higher yield, and less chance of skewed results from different recovery yields. Therefore, total RNA is more often used since relative quantification of the genes of interest (GOI) is more important than the sensitivity of detection of mRNA ${ }^{100}$. Paired with properly designed experiments, $\mathrm{qPCR}$ data can convey changes in gene expression across many treatments groups and communicate trends within these groups.

Before beginning qPCR, the RNA of cells must be isolated. Kits for RNA isolation are readily available from manufacturers, but there are methods that do not require kits which yield high purity RNA. Next, the single stranded RNA must be diluted, to normalize the amount of RNA per treatment group, and made into double stranded complementary DNA (cDNA) for use as template DNA. cDNA is produced by adding RNA, unpaired nucleotides (dNTPs), DNA polymerase, and reverse transcriptase to a thermocycler to create one cDNA molecule for every RNA strand added. This cDNA can then be used for qPCR, where the cDNA, primers specific for each GOI, probes, dNTPs, and DNA polymerase are combined and added to a thermal cycler that heats and cools the samples repeatedly, replicating the template strand. Primers that bind to each replication fluoresce to indicate a new replicate of the GOI, and this fluorescence is 
counted by a camera. The thermocycler repeats this for forty cycles, generating an amplification curve with the amount of fluorescence at each cycle for every sample ${ }^{11,101}$.

As the cycles increase during qPCR, fluorescence linearly increases until a plateau is reached indicating that all dNTPs have been depleted. A common threshold of fluorescent intensity is established to compare the amount of cycles necessary for each sample to achieve that threshold, in which this cycle number is known as the cycles to threshold $(\mathrm{Ct})^{17}$. This $\mathrm{Ct}$ value is the numerical value used in the analysis of the data. The $\mathrm{Ct}$ value is shown in Figure 13 at the intersection of the amplification curve and threshold line.

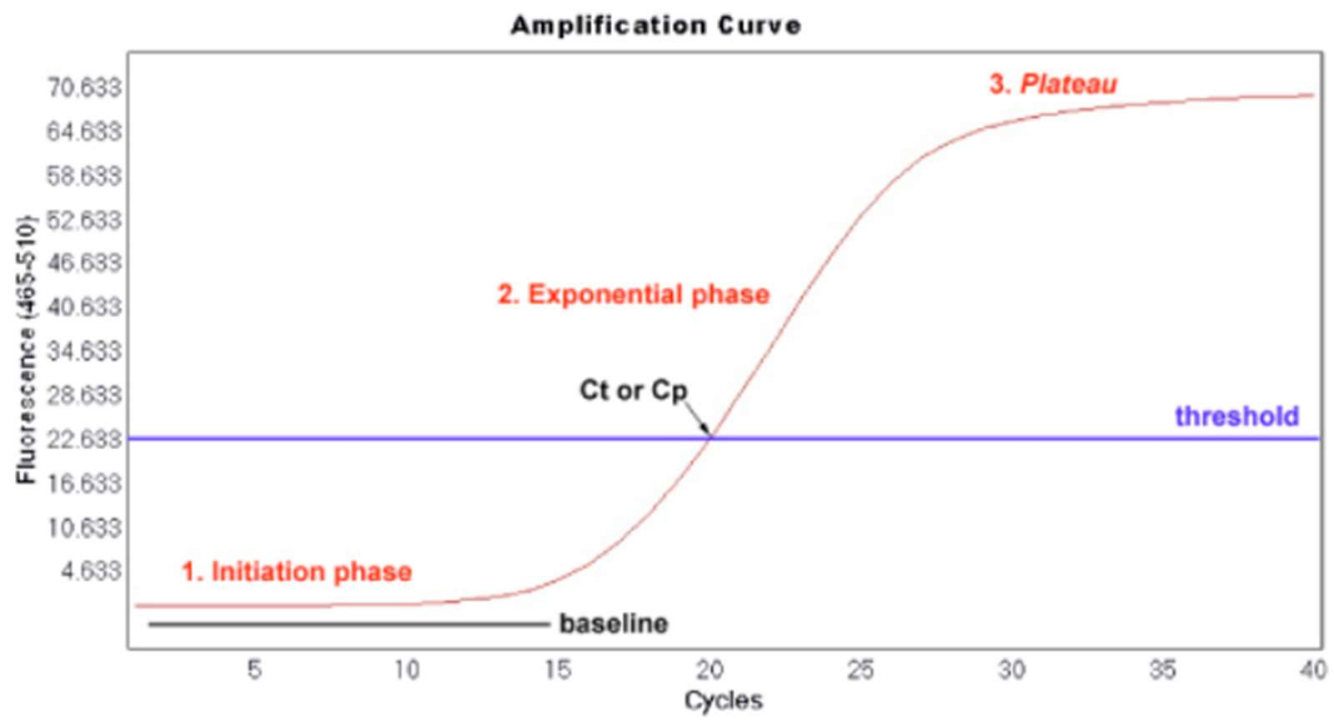

Figure 13: qPCR Amplification Curve. An example amplification curve with the three phases and threshold labeled ${ }^{17}$.

The fluorescence measurement is the "quantitative" portion of qPCR and results from the type of probe used in the primers. Therefore, the function of these probes must be expanded on. Primers are a short single strand of nucleotides that serves as the starting point for DNA synthesis. During each cooling cycle by the thermocycler primers initiate 
the extension of new double stranded DNA (dsDNA) from the single stranded DNA (ssDNA). During this extension probes are utilized to indicate the replication of the template DNA, in which two main types of probes are commonly used: SYBR Green and TaqMan. TaqMan probes, also known as hydrolysis probes, are composed of a fluorescent reporter and quencher attached to a specific nucleotide sequence. During annealing the probe binds to the specific target sequence. As extension occurs, the quencher is cleaved from the probe. The change in proximity of the quencher allows the reporter to fluoresce. These probes, while more specific, are also far more expensive than SYBR Green probes. Therefore, SYBR Green probes were used in all experiments in this thesis. SYBR Green is a DNA-binding dye which binds nonspecifically to any dsDNA. Figure 14 depicts how SYBR Green intercalates within the nucleotides of the extending dsDNA, and this binding event causes fluorescence of the dye ${ }^{102}$.

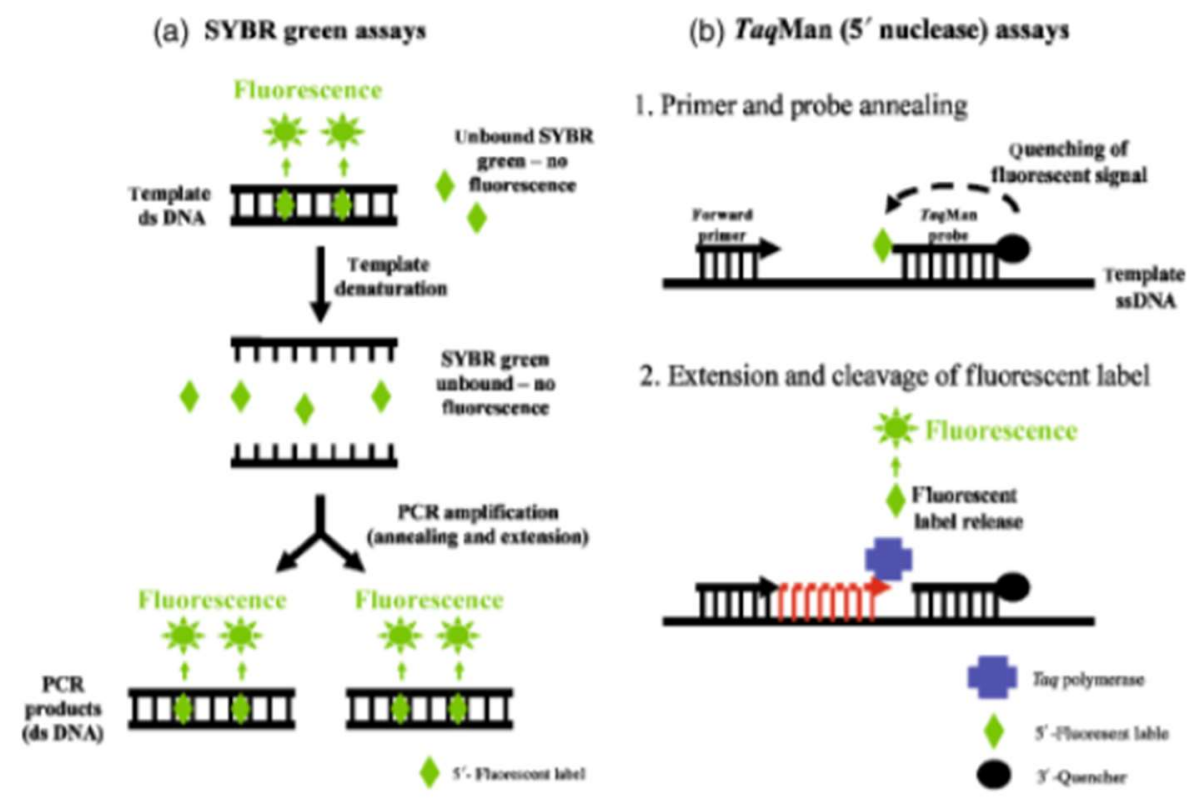

Figure 14: Types of Primers. Depictions of SYBR Green intercalation in double stranded DNA versus TaqMan labeling ${ }^{103}$. 
Since SYBR Green binds to any dsDNA, it is possible to have fluorescence measured when non-template DNA intercalates SYBR Green. One example of this is primer-dimer formation, when two primers hybridize to each other, which leads to false fluorescence measurements. Therefore, a dissociation (or melt) curve must be run when using SYBR Green after the amplification cycle to test that the qPCR assay has yielded single, specific products ${ }^{104}$. The dissociation curve measures the change in fluorescence when the SYBR Green dissociates from the dsDNA as temperature increases ${ }^{101}$. Ideally, qPCR products will melt at the same time, so the resulting change in fluorescence over time will result in a similar curve between samples. These ideal curves are labelled "specific products" in Figure 15.

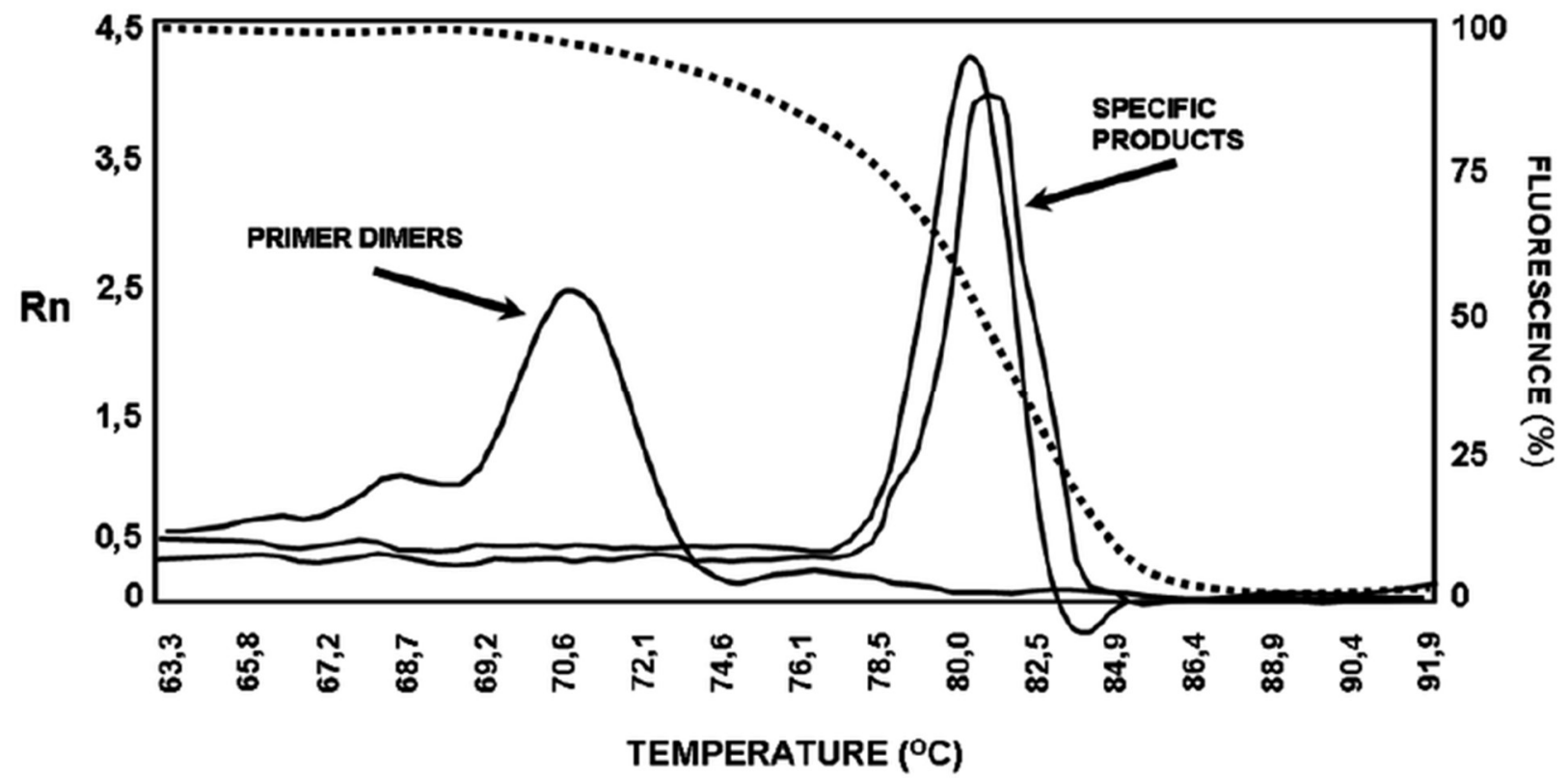

Figure 15: Example Dissociation Curves. Dissociation curves can be used to distinguish PCR products from false signals caused by primer dimers ${ }^{105}$.

Housekeeping genes are necessary for any qPCR experiment in order to analyze the $\mathrm{Ct}$ values. These genes are selected to have stable expression regardless of treatment 
group, which is then used to normalize the $\mathrm{Ct}$ values between experimental groups. While many genes theoretically fit this requirement, there is no agreed upon, consistently stable housekeeping gene. Housekeeping genes must be selected and verified based on the experiment type in which they will be used. Two housekeeping genes were initially selected for Aim 1. The first, $\beta$-actin ( $\beta \mathrm{ACT})$, is one of several types of actin that are a part of the cytoskeleton microfilaments. It is commonly used in experiments regarding EndMT $^{106-109}$. The second, $\beta$ glucuronidase (GUS $\beta$ ), is an enzyme involved in the breakdown of glycosaminoglycans (GAGs) in lysosomes ${ }^{110}$. Both GUS $\beta$ and $\beta$ ACT were shown to be a stable reference gene for experiments using HUVECs when challenged against many other common housekeeping genes ${ }^{111,112}$.

\subsubsection{Summary}

This introduction to Chapter 2 has discussed a variety of topics in order to gain an understanding of all components that apply to this aim. A stark difference between physiologic and BVM flow conditions was established. Normal EC phenotype was defined and compared against the phenotype of ECs undergoing EndMT. A general, yet comprehensive, review of EndMT was provided in order to understand the complexity of EC dedifferentiation in vitro and in vivo. This introduction identified EC markers and mesenchymal markers that can be useful in identifying gene expression changes. Finally, an introduction to $\mathrm{qPCR}$ was presented, which will be the primary analysis method of EC characterization in the following section. The following section will describe the methods that were used in this aim to culture cells, implement a disturbed flow condition, create BVMs, and perform qPCR to evaluate phenotype and EndMT markers in HUVECs. 


\subsection{Materials and Methods}

\subsubsection{Gene Selection and Primer Acquisition}

Many relevant genes were identified and discussed in Section 2.1. This list needed to be narrowed down to identify only a few genes of interest (GOI). To track EndMT progression representative endothelial and mesenchymal markers had to be selected. It was decided that genes coding for the three main proteins of the mechanosensory complex would be used as endothelial markers: PECAM-1, VE-Cadherin, VEGFR2. The genes $\alpha$ SMA and calponin were selected as mesenchymal markers, with $\alpha$ SMA as an early indicator of mesenchymal acquisition and calponin as a late transition marker. The markers $\beta$ ACT and GUS $\beta$ were chosen as housekeeping genes.

With GOI selected, primers for each gene needed to be acquired. Previous projects attempted to design primers, resulting in poor primer selection. Therefore, preverified primers were used throughout all experiments. PrimeTime qPCR primers (IDT, Coralville, IA) were ordered, which include forward and reverse primers that have already been tested for greater than $90 \%$ efficiency. Sequences of the primers, as well as other primer characteristics, can be found in Appendix A.

Primer assays, which include both the forward and reverse primer, were resuspended in $500 \mu \mathrm{L}$ of IDTE buffer for a final desired concentration of 20x. While these preverified primers are guaranteed to have an efficiency of greater than $90 \%$, it was still necessary to test primer efficiency once resuspended to ensure that storage and resuspension had not affected the quality of the primers. Primer efficiency shows how well the target sequence doubles during each replication cycle. If each molecule of target sequence doubles during each cycle, the primer has an efficiency of $100 \%$. It is necessary 
to verify that efficiencies are between $90 \%$ and $110 \%$ to correctly analyze results.

Calculations of gene expression assumes that primer efficiencies are comparable between $\operatorname{primers}^{113}$.

Primer efficiency was calculated by running a logarithmic dilution of cDNA with each primer, then evaluating the slope of the $\mathrm{Ct}$ values for the dilutions. The efficiency (E) percentage was calculated using this slope, which can be seen in Equation 1.

Equation $1 \quad$ Efficiency $(\%)=\left(10^{(-1 / \text { Slope })}-1\right) * 100$

The protocol for primer efficiency testing can be found in Appendix B. PECAM1, VEGFR2, VE-cadherin, and GUS $\beta$ used the same HUVEC cDNA to test for efficiency, while ACTA2 and CNN1 used SMC cDNA. The use of SMC cDNA was necessary since there was not a high enough expression of ACTA2 and CNN1 in the HUVEC cDNA to accurately dilute cDNA and observe a linear decrease in cycles to threshold.

\subsubsection{RNA Isolation}

Several commercial kits are available for RNA isolation. The RNeasy Mini Kit (QIAGEN) has previously been used to yield high purity RNA, so the use of this kit was continued for experiments with cells only (the kit cannot be used for RNA isolation from BVMs). The RNeasy kit uses spin columns to isolate up to $100 \mu \mathrm{g}$ of RNA longer than 200 bases. A high-salt buffer system binds RNA to silica membranes ${ }^{114}$. The samples were first snap-frozen in liquid nitrogen to preserve the RNA and disrupt the cell membranes. The samples were then lysed and homogenized before binding to the 
RNeasy spin column, where multiple wash steps purify the RNases. The final RNA product was eluted from the membranes using water.

Once RNA was isolated, the purity and concentration of the RNA product was assessed. The NanoDrop 2000 Spectrophotometer (Thermo Scientific) was used to measure $2 \mu \mathrm{L}$ RNA samples. The spectrophotometer measures the absorbance of a solution and breaks the signal into functions of wavelength. Measurements on nucleic acid content include concentration, A260, A280, 260/280, and 260/230. The concentration is measured as $\mathrm{ng} / \mathrm{nL}$ based on the absorbance at $260 \mathrm{~nm}$; this measurement is important in order to normalize the amount of RNA added to each reverse transcription reaction. A260 and A280 are the absorbance at 260 and $280 \mathrm{~nm}$, respectively. The $260 / 280$ and $260 / 230$ measurements are ratios of absorbance that indicate the purity of the RNA. A 260/280 ratio of approximately 2.0 is accepted as pure, and values above or below 2.0 indicate a contaminant near $280 \mathrm{~nm}$. The $260 / 230$ ratio is a secondary measurement of purity with an accepted ratio between 1.8 and $2.2^{115}$. A full protocol for RNA isolation using the RNeasy Mini Kit and purity assessment using the NanoDrop 2000 can be found in Appendix C.

\subsubsection{Reverse Transcription}

After verifying that the purity of isolated RNA was acceptable, cDNA was synthesized through reverse transcription. The iScript cDNA Synthesis Kit (Bio-Rad) contains only three components: 5x iScript Reaction Mix, iScript Reverse Transcriptase, nuclease-free water. The reaction mix contains a blend of oligo(dT) and random hexamer primers. The reverse transcriptase is $\mathrm{RNase} \mathrm{H}+$, which improves the amplification 
sensitivity, and contains RNase inhibitors to prevent sample degradation ${ }^{18,116}$. The RNase free water was used to dilute RNA concentrations to maintain constant concentrations across all samples. That way, the same amount of cDNA product will be created, and amplification during qPCR will not be a factor of cDNA concentration. The equation for calculating the volume of RNA to add per sample is shown in Equation 2 for $1000 \mathrm{ng}$ of RNA per sample. The calculated volume of water was subtracted from $15 \mathrm{uL}$ to determine the volume of water needed for the reaction.

Equation $2 \quad(1000 \mathrm{ng} R N A) *(1 \mathrm{~mL} /[\mathrm{RNA}$ concentration $] \mathrm{ng} / \mathrm{nL})=[$ Volume $R N A] \mu L$

The 5x iScript Reaction Mix, reverse transcriptase, nuclease-free water, and RNA were added to a nuclease free tube. Each reaction contains a total of $20 \mu \mathrm{L}$ per well. After loading the samples in a thermocycler, the samples were heated and cooled for one cycle to produce the double-stranded cDNA. The samples were then diluted with $80 \mu \mathrm{L}$ of nuclease-free water and stored at $-20 \mathrm{C}$. The full protocol for cDNA synthesis can be found in Appendix D.

\subsubsection{Quantitative Polymerase Chain Reaction}

Once ds cDNA was synthesized and diluted, qPCR was performed. SYBR Green dye was used for the reactions, in which the Fast SYBR Green Master Mix (Life Technologies) was used. This master mix contains SYBR Green dye, DNA polymerase, uracil-DNA glycosylase, ROX passive reference dye, and dNTPs ${ }^{117}$. In a 96-well plate, 4 $\mu \mathrm{L}$ SYBR Green Master Mix, $3.2 \mu \mathrm{L}$ cDNA, $1 \mu \mathrm{L}$ primers, and $11.8 \mu \mathrm{L}$ of water was loaded in each well for a total reaction volume of $20 \mu \mathrm{L}$. Triplicates of each sample were 
run, as well as non-template controls (NTCs) for each primer. NTCs contain no cDNA (the volume of cDNA is replaced by water) and are included to monitor for contamination and primer-dimer formation, both of which could produce false positive results $^{118}$.

Once loaded into the well plate, the plate was placed into the qPCR 7500 Fast System (Applied Biosystems). A standard run time was selected, to which the cycle settings can be found in Appendix E, and a dissociation cycle was added. After the cycles were complete, the $\mathrm{Ct}$ values were exported for analysis. Several assumptions must be verified for different qPCR analysis methods. The method selected for this thesis, relative abundance, assumes a primer efficiency with an efficiency of 2 and a stable reference gene were used. The assumption of primer efficiency was checked and verified as described in Section 2.2.1, and the assumption of using a stable reference gene was checked after each experiment. Relative abundance was measured by the $2^{-\Delta \mathrm{Ct}}$ value, where $\Delta \mathrm{Ct}$ is calculated as shown by Equation 3.

Equation $3 \quad \Delta C t=C t(G O I)-C t($ reference gene $)$

\subsubsection{Experiment 1 Pilot: Change in EC Expression with Increasing Passage}

\section{Number}

As previously established, the goal of Aim 1 was to determine how endothelial cell-gene expression changes in respect to passage number and flow conditions, with the understanding that increased time in culture and disturbed flow should contribute to EndMT. The effect of increasing time in culture was initially investigated. This preliminary pilot served to establish an understanding of how gene expression changed 
during just a few passage numbers, as well as to check the stability of the housekeeping genes as passage number increased. RNA was isolated from HUVECs at passages 4, 6, 7, 8,9 , and 10, cDNA synthesized, and qPCR run. Relative abundance was calculated, and this data, along with the results of the dissociation cycle, were analyzed. Ct values for the housekeeping genes $\beta \mathrm{ACT}$ and GUS $\beta$ were also examined in order to determine the viability of the genes as housekeepers.

\subsubsection{Experiment 1: Change in EC Expression with Increasing Passage Number}

Experiment 1 served as a more thorough investigation of the goals established by the Experiment 1 Pilot. The goal of the experiment was still to examine changes in gene expression as culture time increased as well as to add more passage number conditions for the HUVECs. This experiment also added SMCs at early and late passages to compare mesenchymal marker expression to endothelial expression of the same markers. RNA was isolated from HUVECs at passage 3, 5, 7, 9, 11, and 13, whole RNA was isolated from SMCs at passage 6 and 11.

\subsubsection{Experiment 2: Changes in EC Expression from Disturbed Flow Conditions}

Experiment 2 investigated the effect of disturbed flow on EC gene expression; disturbed flow should induce changes in gene expression towards a mesenchymal state. In order to mimic disturbed flow conditions, 6-well plates of HUVECs were secured to an orbital shaker in an incubator and allowed to run at 210 RPM for 24 hours. This technique was borrowed from Mahmoud et al., which found that the orbital shaker caused low shear stress (about 5 dyne $/ \mathrm{cm}^{2}$ ) with variations in direction in the center of the 6- 
well, and high shear stress (about $15 \mathrm{dyne} / \mathrm{cm}^{2}$ ) at the edge of the 6-well with uniform flow direction ${ }^{69}$. However, since it was unknown to what extent these conditions would cause changes in gene expression towards a mesenchymal phenotype, a positive control was necessary. Therefore, HUVECs were cultured in SMC specific media for 48 hours, which will induce phenotypic changes in HUVECs towards a mesenchymal phenotype. This would serve as the positive control for which to compare the HUVECs exposed to disturbed flow. HUVECs cultured in static conditions were used as a no-treatment group. The static, disturbed flow, and SMC-media conditions included passage numbers 6, 10, and 14.

\subsubsection{Experiment 3 Preparation: Development of RNA Isolation Protocol for BVMs}

The previous experiments used only cells cultured in dishes. In order to investigate the changes in gene expression of cells in a BVM, the RNeasy Mini Kit for RNA isolation could not be used. Therefore, a new isolation protocol needed to be developed and verified for high purity and appropriate concentration RNA. One method to isolate RNA from BVMs was to homogenize the BVM, scaffold and cells, into a solution and then isolate the RNA. Trizol (Life Technologies) isolation is a common procedure in which tissue is homogenized in Trizol and then RNA is extracted from that solution. This method was pursued for RNA isolation from BVMs.

Many protocols for Trizol isolation are available, and most have only minor differences. A reliable protocol that had been used previously at an internship was employed for the isolation in this thesis, and can be found in Appendix C. The BVMs were frozen in liquid nitrogen directly after removal from bioreactors and stored at $-80 \mathrm{C}$ 
until use. The BVMs were then cut into small pieces using scissors, and $5 \mathrm{~mL}$ of Trizol was added before homogenizing the BVM in the Trizol using a TissueRuptor (Qiagen). Chloroform was added to the homogenate, and the solution was centrifuged to separate a Trizol and aqueous phase. The aqueous phase, which contains the RNA, was removed and added to 99\% IPA. GlycoBlue (Thermo Fisher Scientific) was also added to the solution before centrifugation. The supernatant was removed, leaving only a blue pellet

containing RNA in the bottom of the centrifuge tube. The pellet was resuspended in $75 \%$ EtOH and stored at $-80 \mathrm{C}$ overnight. The following day the tube was centrifuged, and the supernatant removed. The pellet dried completely before resuspending in nuclease-free water. The RNA purity and concentration were checked as described in Section 2.2.2. In order to compare the product of this isolation protocol against the RNA isolation from the RNeasy Mini Kit, both protocols were used for the isolation of RNA from HUVECs. The purity and concentrations were then compared to determine whether the Trizol isolation was comparable to the RNeasy isolation.

\subsubsection{Experiment 3 Pilot: Changes in Gene Expression in BVMs}

Having already investigated HUVECs in static culture and in disturbed flow, as well as having developed an RNA isolation protocol for use on BVMs, the proper criteria had been met to investigate gene expression changes in BVMs. This pilot experiment used only two BVMs that ran for 24 hours. A static condition and a disturbed flow condition were also used to compare all three flow types. The passage number was kept constant in this pilot to remove the variability of expression as cells remain in culture for 
different lengths of time. The three flow condition groups (BVM, static, and disturbed flow) used HUVECs at passage number 6.

The disturbed flow condition with HUVECs in 6-well plates underwent a ramp-up period on the orbital shaker. This change was implemented as a result of a high percent of cell death in the disturbed flow groups in Experiment 2. The ramp-up period increased the orbital shaker from 50 RPM to 200 RPM at intervals of 50 RPM every 2 hours. The plates remained at 200 RPM for 24 hours.

\subsection{Results}

\subsubsection{Primer Efficiency}

Preverified primers should not need to be checked for efficiency; however, appropriate efficiencies are necessary to meet the assumptions for relative abundance calculations. A representative graph of primer efficiency is shown in Figure 16, and all efficiency plots can be found in Appendix G. Slope, $\mathrm{R}^{2}$, and efficiency percentage values for all GOI can be found in Table 2. Efficiency percentages ranged from $86.5 \%$ to $99.5 \%$, with GUS $\beta$ having the lowest efficiency. An efficiency range between $90 \%$ and $110 \%$ is adequate to meet the assumptions for relative abundance, of which VEGFR2 and GUS $\beta$ fell outside of this range at $86.5 \%$ and $88.6 \%$, respectively. All $\mathrm{R}^{2}$ values were above 0.97, in which this linearity indicates pipetting accuracy for the dilutions. The efficiency for $\beta \mathrm{ACT}$ was not calculated, as this gene had already been eliminated as a viable housekeeping gene at the time of efficiency testing. This decision will be discussed in Section 2.4 . 


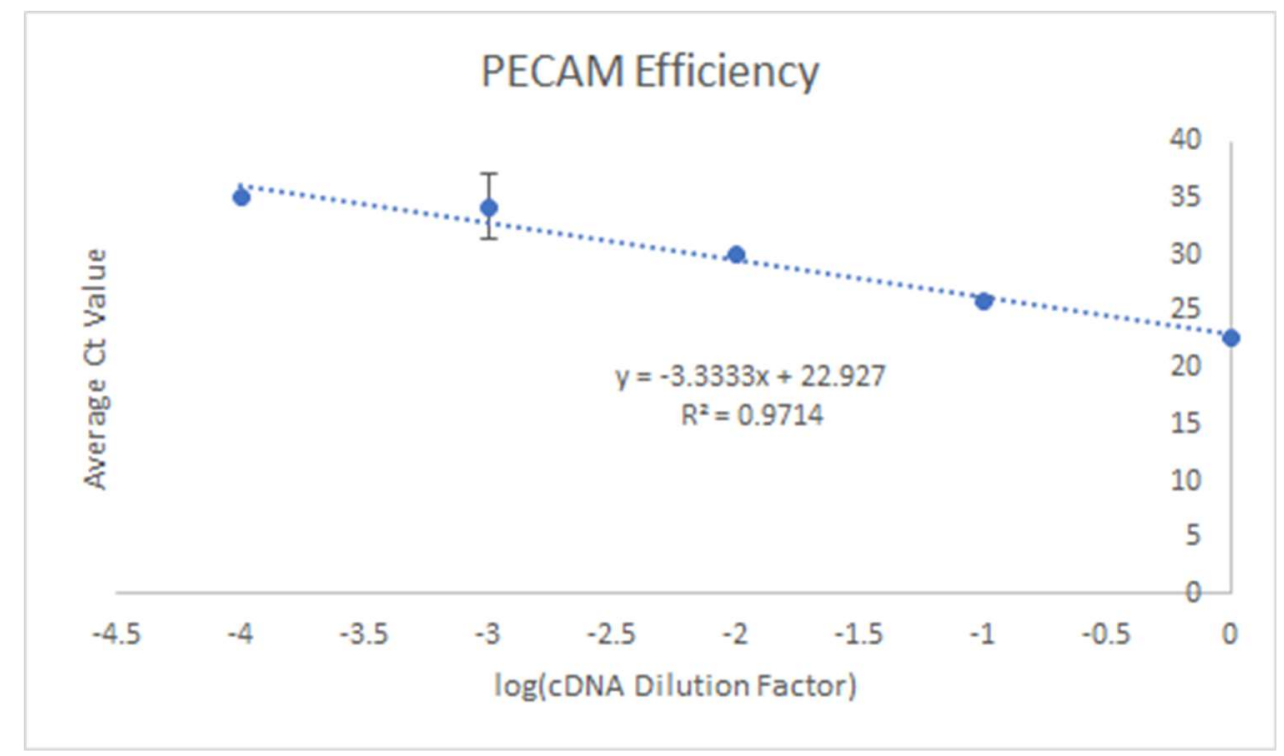

Figure 16: Primer Efficiency Plot. Representative primer efficiency graph of the log of the cDNA dilution factor versus the average $\mathrm{Ct}$ value. The slope of the trendline is used for efficiency calculations.

Table 2: Primer Efficiency. Efficiency data from GOI, which includes the slope, $\mathrm{R}^{2}$, and efficiency percentage.

\begin{tabular}{l|lll}
\hline \multicolumn{2}{l}{ Slope } & \multicolumn{1}{l}{$\mathbf{R}^{2}$} & \multicolumn{1}{c}{ Efficiency $(\%)$} \\
\hline PECAM & -3.3333 & 0.9714 & 99.52761 \\
VEGFR2 & -3.6301 & 0.999 & 88.57083 \\
VE-Cadherin & -3.4575 & 0.9988 & 94.63741 \\
ACTA2 & -3.3482 & 0.9943 & 98.91519 \\
CNN1 & -3.4528 & 0.9848 & 94.81393 \\
GUS $\beta$ & -3.6939 & 0.9979 & 86.51621
\end{tabular}

\subsubsection{Experiment 1 Pilot Results}

This pilot served to investigate the effect of static culture time on gene expression in HUVECs, evaluate $\beta$ ACT and GUS $\beta$ as housekeeping genes, and accumulate data in order to better design Experiment 1. Figure 17A shows relative abundance of all GOI as 
compared to $\beta \mathrm{ACT}$. VE-cadherin had the highest abundance among the GOI, followed by VEGFR2. A slight trend can be seen for both VE-cad and VEGFR2, with increasing abundance as passage number increased. VEGFR2 followed this trend except at passage 6. VE-cad had a maximum abundance at passage 8 , where abundance slightly decreased at passages 9 and 10. The high abundance of VE-cad made identifying trends in PECAM, ACTA2 and CNN1 expression difficult, so Figure 17B shows the same data as Figure 17A but excluded VE-cad and VEGFR2 data. Interestingly, PECAM and ACTA2 had similar abundance in HUVECs regardless of the passage number. Calponin had almost no expression. 


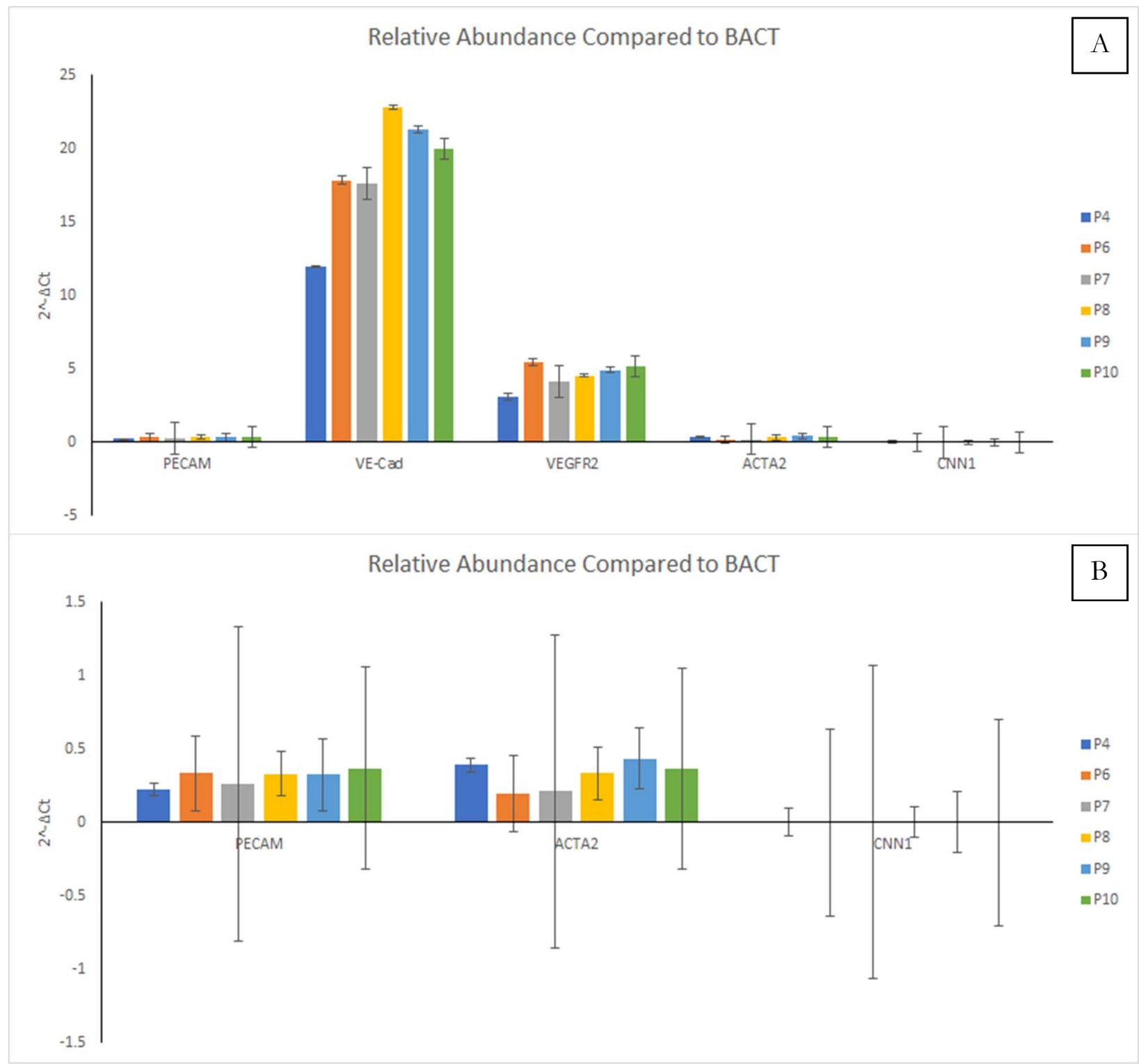

Figure 17: Experiment 1 Pilot Relative Abundance Relative to $\beta$-Actin. (A) Relative abundance of all genes of interest using $\beta$-Actin ( $\beta$ ACT) as the housekeeping gene. (B) Platelet Endothelial Cell Adhesion Molecule (PECAM), Alpha Actin (ACTA2), and Calponin $(\mathrm{CNN} 1)$ relative abundance using $\beta \mathrm{ACT}$ as the housekeeping gene.

Figure 18A shows relative abundance levels of all GOI compared to GUS $\beta$ as the housekeeping gene. Like the previous figure, Figure 18B shows the same data as Figure 18A but excludes VE-cad and VEGFR2. The trends of VE-cad and VEGFR2 relative to GUS $\beta$ followed closely with the trends compared to $\beta$ ACT. However, the abundance 
levels compared to GUS $\beta$ were about half that as when compared to $\beta$ ACT. PECAM and ACTA2 also had lower abundance levels when compared to GUS $\beta$ than $\beta$ ACT. No trend persisted for PECAM or ACTA2 as passage number increases. Furthermore, calponin had almost no expression.

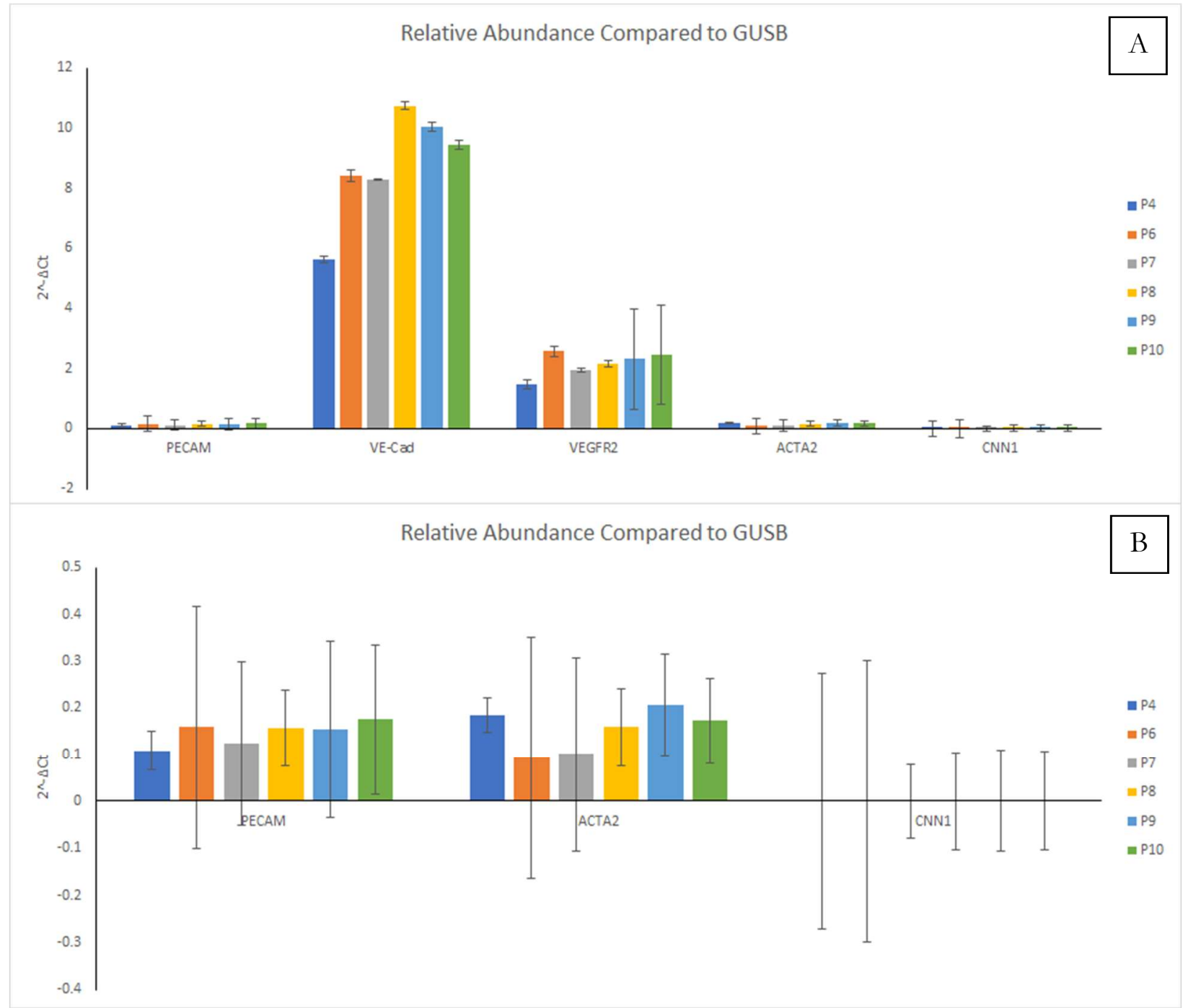

Figure 18: Experiment 1 Pilot Relative Abundance Relative to $\beta$ Galactosidase. (A) HUVEC relative abundance of all genes of interest using $\beta$ Galactosidase (GUS $\beta$ ) as the housekeeping gene. (B) HUVEC relative abundance of PECAM, ACTA2, and CNN1 using GUS $\beta$ as the housekeeping gene. 
It is important to check for the stable expression of housekeeping genes during every experiment to determine whether new treatment groups affect the genes viability as a housekeeper. Figure 19 shows plots of the average $\mathrm{Ct}$ for $\beta \mathrm{ACT}$ and GUS $\beta$ at each passage number. The $\mathrm{Ct}$ average for $\beta \mathrm{ACT}$ was variable between groups, ranging from 20.87 and 24.10 cycles. GUS $\beta$ displayed more stable $\mathrm{Ct}$ values, which range between 20.77 and 21.26 cycles.
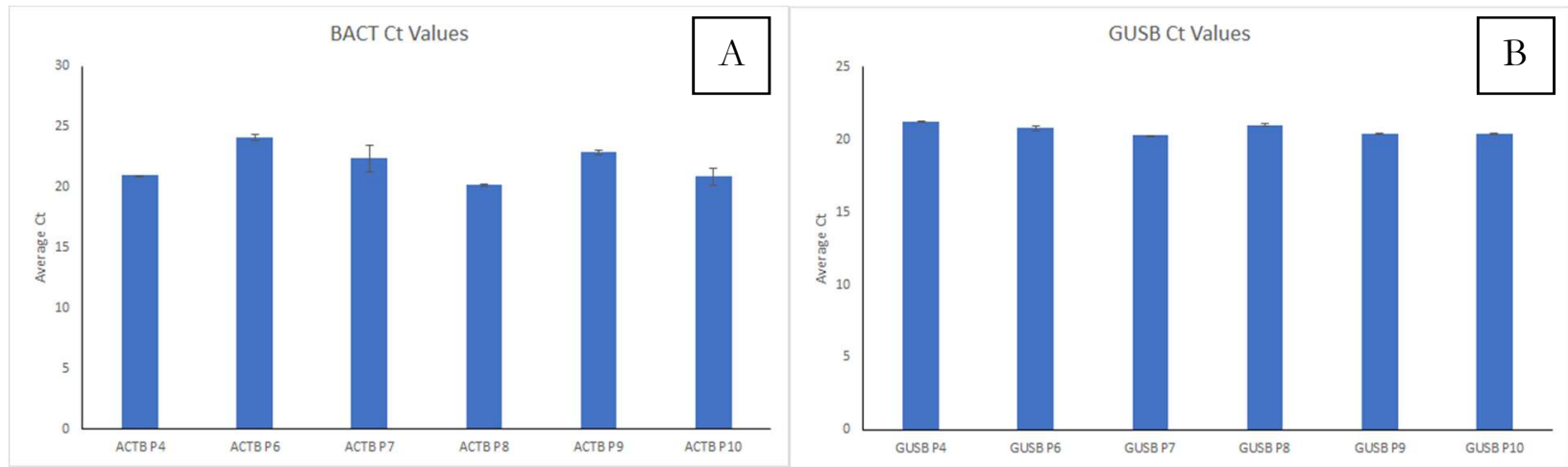

Figure 19: Experiment 1 Pilot Housekeeping Gene Stability. Average Ct values of (A) $\beta \mathrm{ACT}$ and (B) GUS $\beta$ from each treatment condition.

\subsubsection{Experiment 1 Results}

Based on the results of the previous pilot study, Experiment 1 increased the range of passage numbers in order to accentuate any trends that were previously identified and to reveal other trends that may exist in HUVECs in static culture. Experiment 1 also added an early and late passage of HUASMCs to compare the expression of mesenchymal markers between HUVECs and HUASMCs. Since $\beta$ ACT Ct values were more variable than GUS $\beta$ in the pilot, expression of the housekeeping genes were analyzed first before analyzing relative abundance data. Figure 20 displays how $\beta$ ACT $\mathrm{Ct}$ values fluctuated between 16.83 and 25.46 cycles, while GUS $\beta$ Ct values remained relatively stable between 17.44 and 20.37 cycles. As a result of the instability of $\beta$ ACT 
identified in the pilot and this experiment, $\beta \mathrm{ACT}$ was eliminated as a housekeeping gene. From this point forward, only GUS $\beta$ was used while running $\mathrm{qPCR}$ and during data analysis.
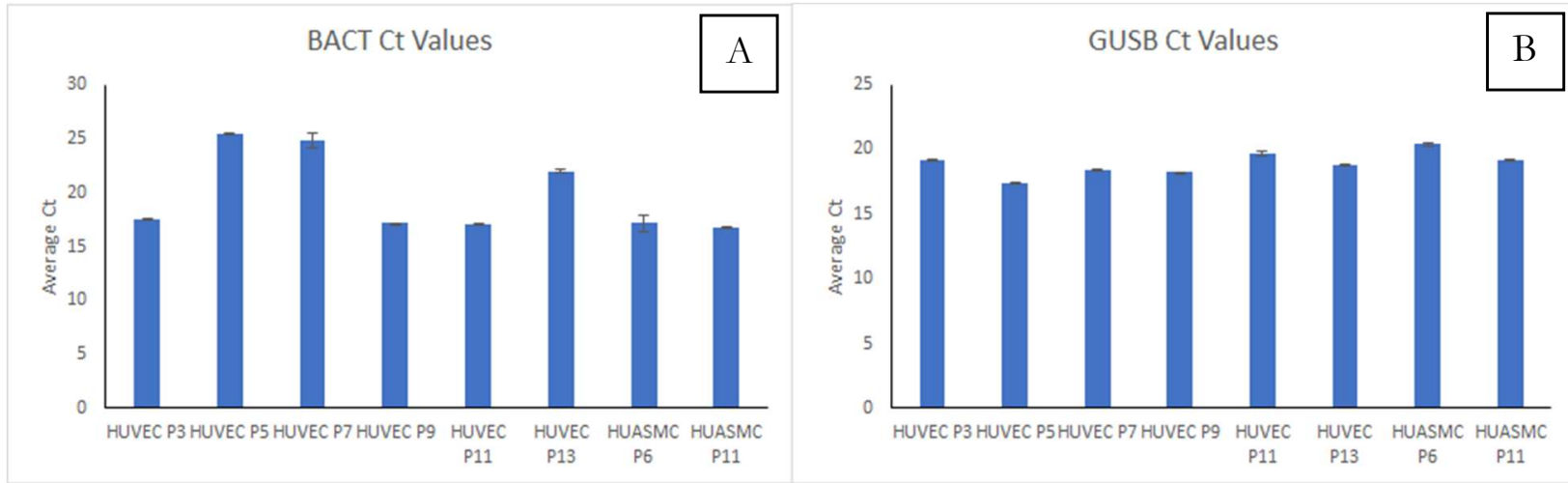

Figure 20: Experiment 1 Housekeeping Gene Stability. Average Ct values of (A) $\beta \mathrm{ACT}$ and (B) GUS $\beta$ from each treatment condition.

Figure 21A shows the relative abundance of all GOI in HUVECs of increasing passage number in static culture. VE-cad and VEGFR2 had a positive trend between relative abundance and passage number. Consistent with the results of the pilot study, these two EC markers were more highly expressed than PECAM. No trends were identified in expression of PECAM and ACTA2, as seen in Figure 21B. Calponin abundance was negligible in HUVECs. 

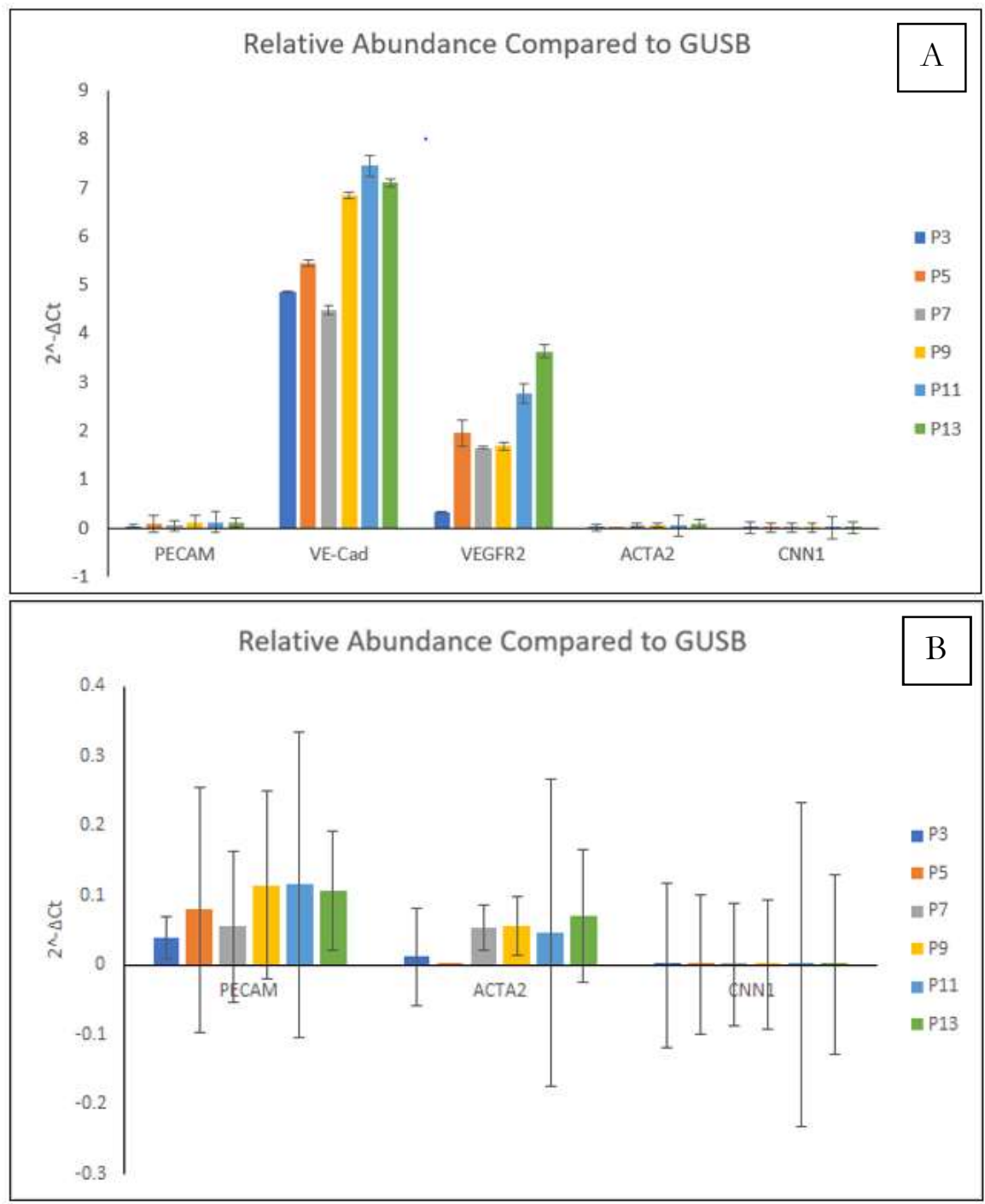

Figure 21: Experiment 1 Relative Abundance. HUVEC relative abundance of (A) all GOI and (B) PECAM, ACTA2, and CNN1 using GUS $\beta$ as the housekeeping gene.

Relative abundance of mesenchymal markers ACTA2 and calponin in early passage (passage 6) and late passage (passage 11) HUASMCs in static culture are shown in Figure 22. ACTA2 was highly expressed compared to calponin in HUASMCs. Expression of ACTA2 and calponin in HUVECs was almost negligible when compared to expression in HUASMCs. 


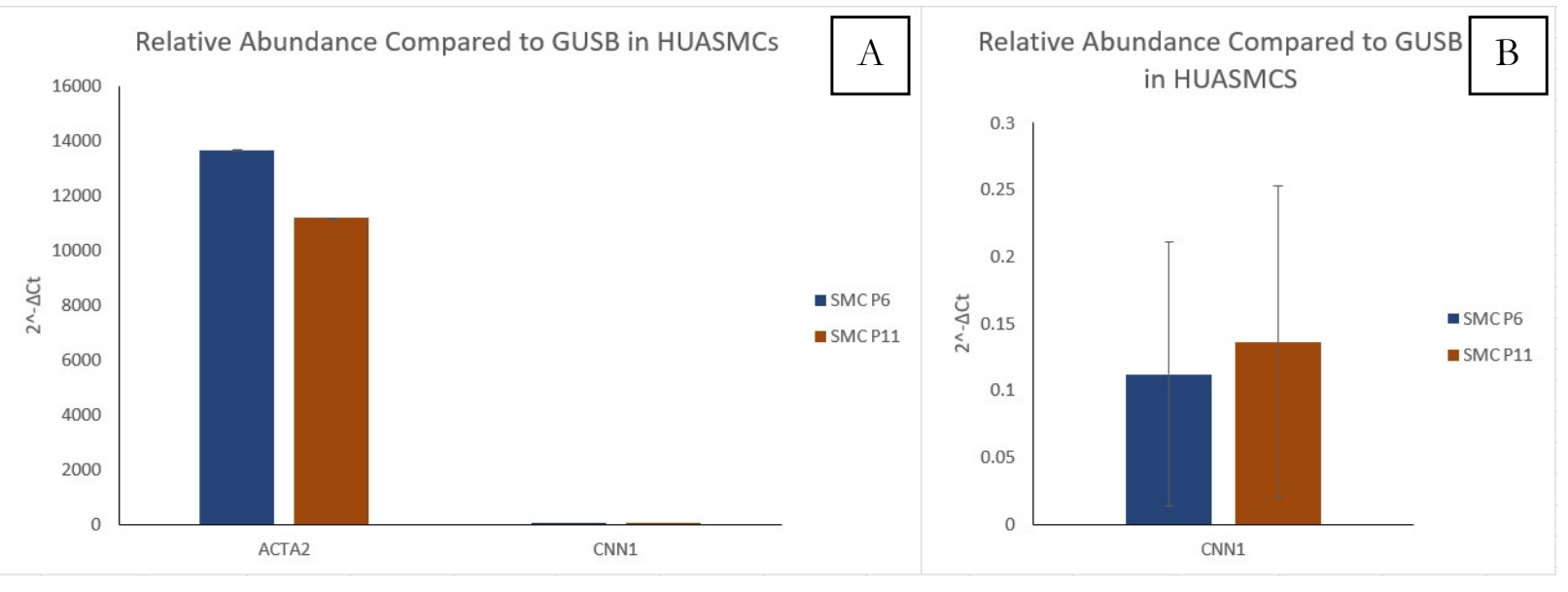

Figure 22: Experiment 1 Pilot Relative Abundance of Mesenchymal Markers in SMCs. (A) HUASMCs relative abundance of ACTA2 and CNN1 using GUS $\beta$ as the housekeeping gene. (B) HUASMC relative abundance of CNN1 using GUS $\beta$ as the housekeeping gene.

SYBR Green dye will intercalate any double stranded DNA, including primerdimers and contaminating DNA. Dissociation curve analysis will detect whether primerdimers or contaminating DNA are present in the qPCR product, and in this way is a check of quality for a single product. A dissociation curve for a product will have a single peak at the same temperature for genes of the same type regardless of treatment. Figure 23 displays examples dissociation curves for each GOI. Dissociation curves were analyzed prior to the analysis of each data set to identify individual samples or entire groups that had abnormal dissociation curves. 

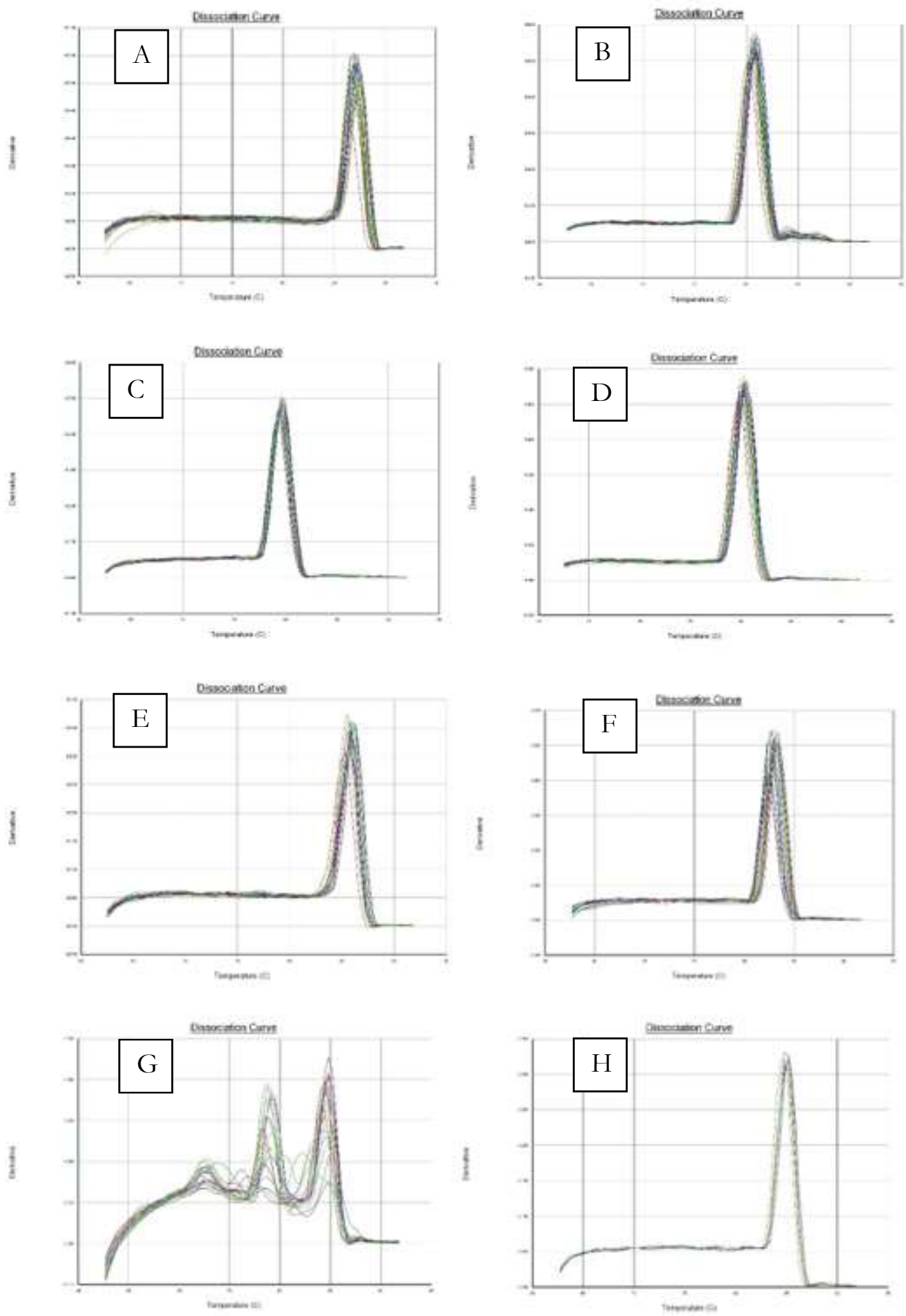

Figure 23: Representative dissociation curves of all GOI. (A) $\beta$ ACT, (B) GUS $\beta$, (C) VEGFR2, (D) VE-cad, (E) PECAM, (F) ACTA2, (G) calponin from ECs, (H) calponin from SMCs. 
Dissociation curves appeared acceptable for all except one GOI. Figure 23G shows the dissociation curve from PCR products for calponin from ECs. These curves had several peaks and low derivative values, indicating poor quality of PCR product. However, this most likely occurred because not enough product could be produced since the abundance of calponin was so low in ECs. The dissociation curve for calponin from SMCs, shown in Figure 23H, showed a single peak. Therefore, the primer was still verified to be working properly and producing high quality PCR products. Figure $23 \mathrm{~A}-\mathrm{F}$ shows single peaks for all other GOI.

\subsubsection{Experiment 2 Results}

While some trends were identified between EC marker expression and passage number in Experiment 1, these results are not as meaningful unless compared against conditions that are known to induce EndMT in ECs. Two treatment groups were added in Experiment 2 in which to compare the non-treatment group of HUVECs cultured in static EC media: HUVECs cultured in disturbed flow, and HUVECs cultured in static SMC media. In each group three passage numbers were chosen to investigate the effect of culture time.

The relative abundance of all GOI are shown in Figure 24A. Unfortunately, due to high amounts of cell death in the disturbed flow treatment groups, a high enough concentration of RNA was only isolated from HUVECs at passage 14. The RNA concentrations at passages 6 and 10 were too low to continue those groups in the experiment. 
VE-cad and VEGFR2 expression increased with passage number in the nontreatment group, while the expression of these two markers decreased with passage number in the SMC media treated group. VE-cad expression of the disturbed flow treatment was lower than that of any non-treatment groups but was higher than the passage 14 SMC media treated group. VEGFR2 expression showed a slight positive trend between culture time and relative abundance in the non-treatment group, and a slight negative trend in the SMC media treatment group. However, the SMC media treatment group had a higher relative abundance at all passages than the non-treatment passage 6 and 10. The disturbed flow treatment had a lower relative abundance of VEGFR2 than all other groups.

PECAM expression was slightly lower in the disturbed flow and SMC mediatreated groups, but not statistically. There was no difference in ACTA2 expression between the non-treatment group and the disturbed flow group. ACTA2 expression trended towards increasing with passage time in the SMC media treated group. Calponin expression was negligible in all groups. 


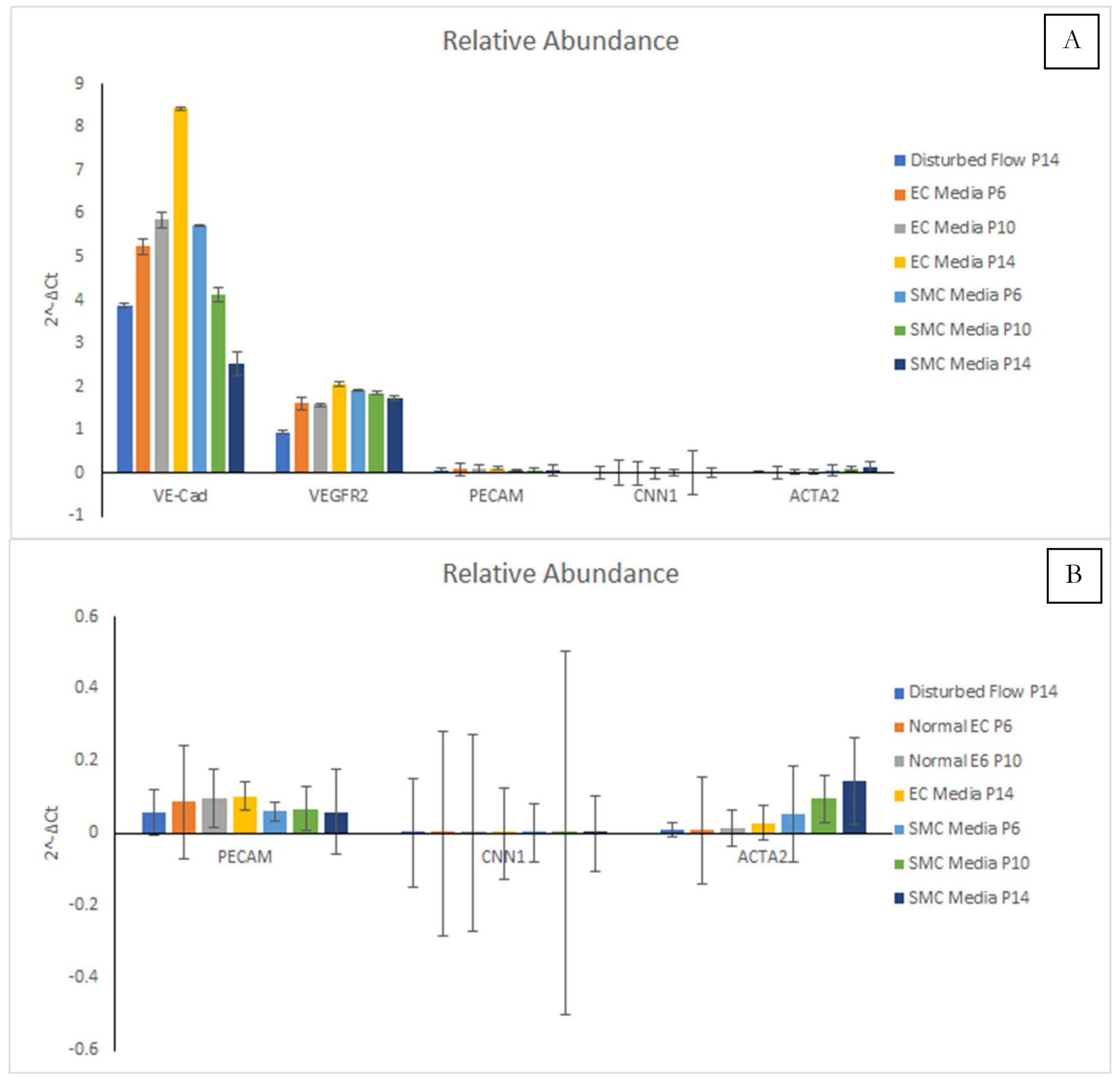

Figure 24: Experiment 2 Relative Abundance. HUVEC relative abundance of (A) all GOI and (B) PECAM, ACTA2, and CNN1 using GUS $\beta$ as the housekeeping gene.

Since two treatment groups were included in Experiment 2, analysis of the stability of GUS $\beta$ was important to determine whether the treatments affected the viability of GUS $\beta$ as a housekeeper. Figure 25 shows that GUS $\beta$ expression remained relatively constant through all treatments, ranging between 19.62 and 20.45 cycles. 


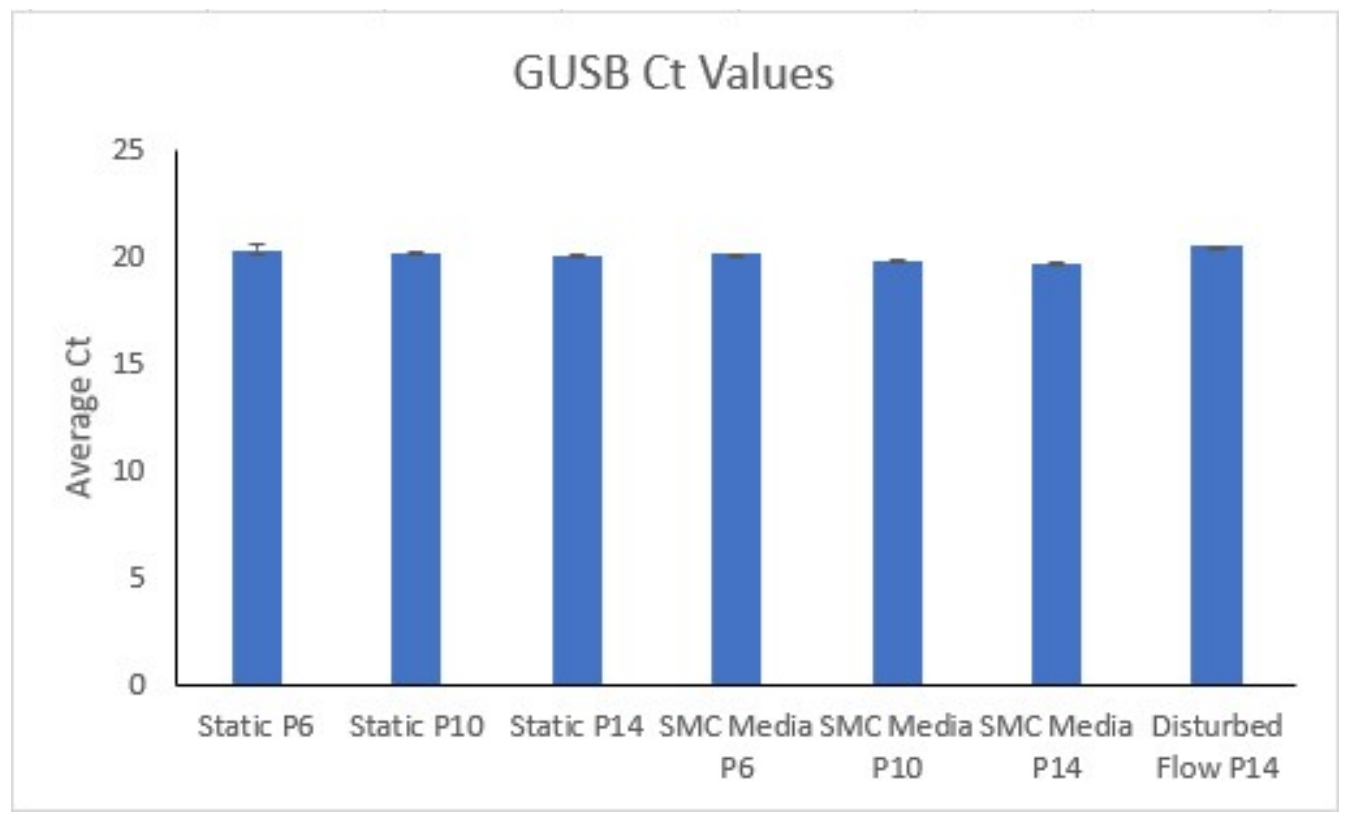

Figure 25: Experiment 2 Housekeeping Gene Stability. Average Ct values of GUS $\beta$ from each treatment condition.

\subsubsection{Verification of RNA Isolation Protocol for BVMs}

A new method had to be developed to be able to isolate RNA from BVMs. It was necessary to verify that this extraction protocol rendered equivalent RNA as the RNeasy Mini Kit. The resulting concentrations, 260/280, and 260/230 values from both isolation processes are shown in Table 3 . The concentration of RNA from the Trizol extraction did not differ from the Mini Kit $(p>0.05)$. The 260/280 and 260/230 ratios did not differ between the isolation methods ( $\mathrm{p}>0.05)$, and the purity average of both methods was within the accepted range. Based on this, the Trizol extraction method was deemed acceptable for BVM RNA isolation. 
Table 3: Comparison of RNA isolation from the RNeasy Mini Kit and Trizol extraction.

\begin{tabular}{l|llllll}
\hline & $\begin{array}{l}\text { RNeasy } \\
\text { Mini Kit } \\
1\end{array}$ & $\begin{array}{l}\text { RNeasy } \\
\text { Mini Kit }\end{array}$ & $\begin{array}{l}\text { RNeasy } \\
\text { Mini Kit } \\
\text { Average }\end{array}$ & $\begin{array}{l}\text { Trizol } \\
\text { Extraction } \\
1\end{array}$ & $\begin{array}{l}\text { Trizol } \\
\text { Extraction } \\
2\end{array}$ & $\begin{array}{l}\text { Trizol } \\
\text { Extraction } \\
\text { Average }\end{array}$ \\
\hline $\begin{array}{l}\text { Concentration } \\
(\text { ng/nL) }\end{array}$ & 2682.9 & 2593.9 & 2638.4 & 2604.3 & 2655.7 & 2630.0 \\
$260 / 280$ & 2.03 & 2.06 & 2.045 & 1.97 & 2.01 & 1.99 \\
$260 / 230$ & 2.18 & 2.24 & 2.21 & 2.07 & 2.19 & 2.13
\end{tabular}

\subsubsection{Experiment 3 Pilot Results}

Experiments 1 and 2 worked to investigate gene expression of HUVECs in static and disturbed flow conditions. With those conditions established, Experiment 3 served to explore gene expression in the laminar flow conditions of BVMs in bioreactors. Before performing an entire study, a pilot was completed which included the three flow conditions but controlled for passage number. Gene expression was analyzed for passage 6 HUVECs in static culture, disturbed flow, and laminar flow. Since a high amount of cell death occurred in Experiment 2 for the disturbed flow condition, a ramp-up period was employed to reduce the shock of the flow on the cells. Figure 26 displays white light images of HUVECs after the ramp-up period and culture for 24 hours at 200 RPM. The well plate remained confluent at the center and edges, indicating that the ramp-up period reduced cell death. Cells at the center of the well were oriented randomly, while cells at the edge of the well were highly aligned and stretched in the direction of flow. 


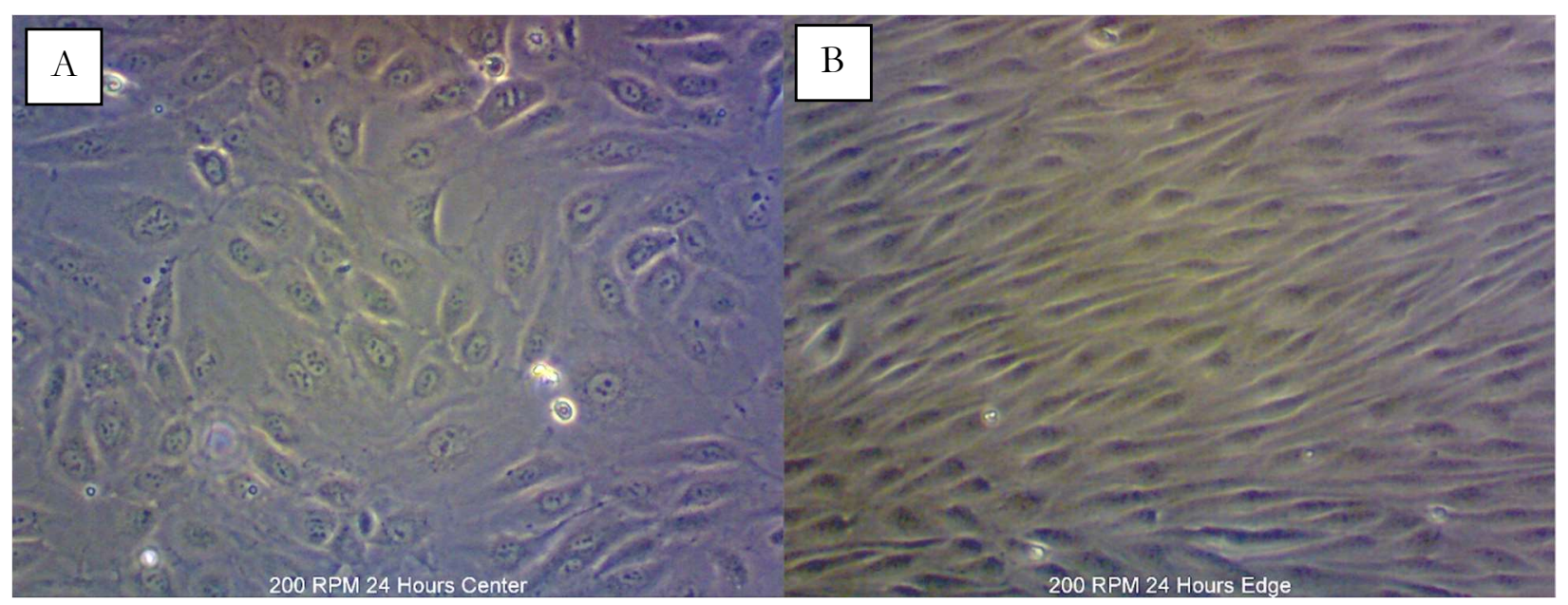

Figure 26: HUVEC Morphology in Turbulent Flow Condition. HUVECs in 6-well plates after 24 hours on a rotator plate at 200 RPM. (A) HUVECs in the center of the well are not aligned. (B) HUVECs on the edge of the well plate align in the direction of flow.

Since a new treatment condition was added to this experiment, $\mathrm{Ct}$ data had to be analyzed to determine whether the treatment affected the utility of GUS $\beta$ as a housekeeper. GUS $\beta$ Ct values are plotted for each treatment group in Figure 27, which makes clear that the $\mathrm{Ct}$ values varied based on treatment. The non-treated, static culture condition had a $\mathrm{Ct}$ average of 19.9 , which was similar to the results seen in previous experiments. The $\mathrm{Ct}$ values for both BVMs differed, with vessel 8 and 9 having $\mathrm{Ct}$ values of 27.72 and 23.57 , respectively. Surprisingly, the disturbed flow treatment had a Ct of 39.49, even though the Ct average for the same condition in Experiment 2 was 20.45. 


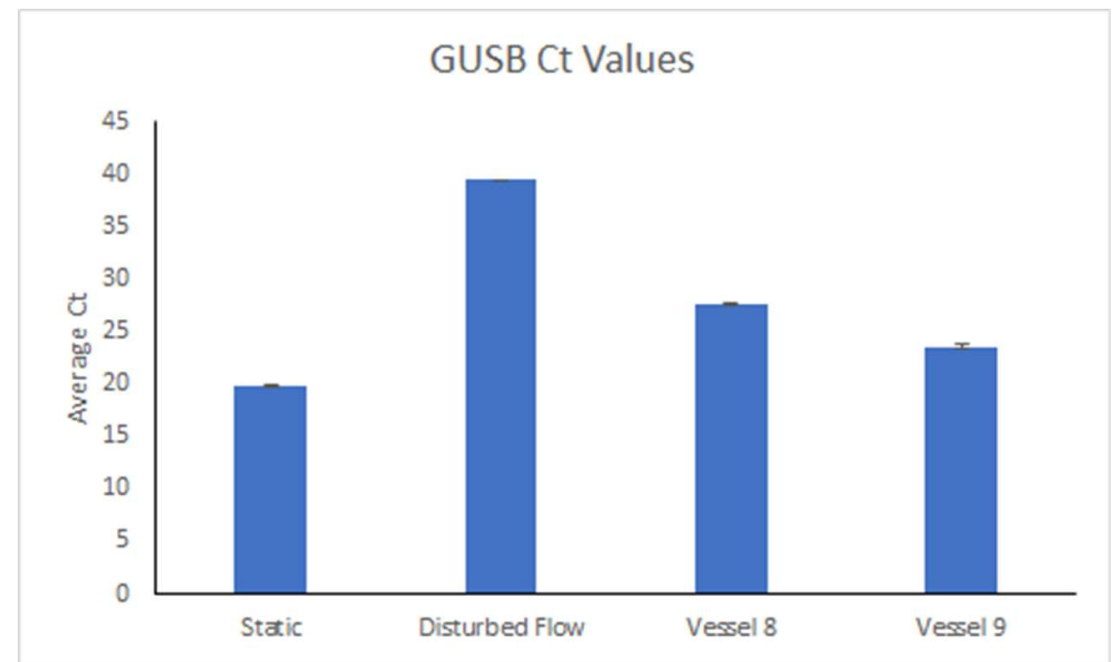

Figure 27: Experiment 3 Housekeeping Gene Stability. Average Ct values of GUS $\beta$ from each treatment condition.

Upon consulting the dissociation curves, it was observed that all GOI in the turbulent flow condition, excluding PECAM, had multiple peaks. Therefore, these samples were excluded from the study. Only PECAM had a melt curve with a single peak, which can be seen by the peak on the right side of Figure 28. However, the abundance value was over 1700 for PECAM, making it an obvious outlier. Therefore, this sample was also excluded from the study, so the entire disturbed flow condition was not included in the results. In doing so, the Ct values of GUS $\beta$ were more stable. An average between GUS $\beta$ values for the static condition and vessels was used for normalizing the data. 


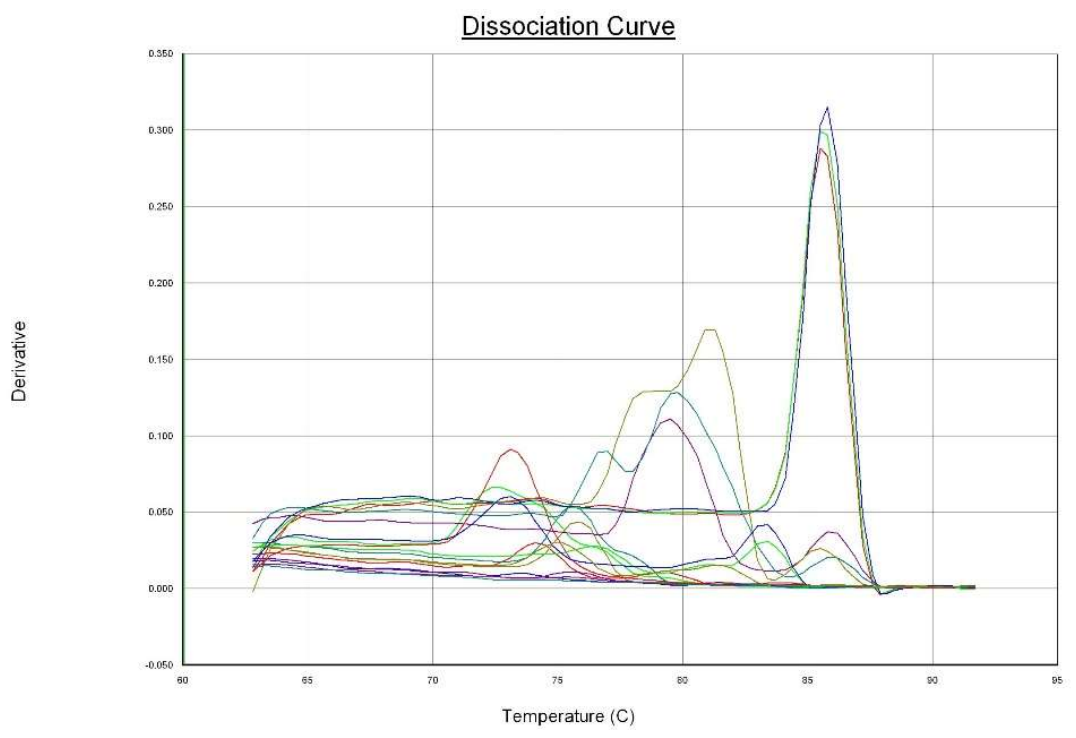

Figure 28: Disturbed Flow Dissociation Curves. Dissociation curve of all GOI for the disturbed flow condition in Experiment 3.

The static condition had higher expression of both VEGFR2, VE-cad, and PECAM than the two BVMs, as shown in Figure 29. The BVMs had comparable expression of VEGFR2, PECAM, and ACTA2. Only VE-cad was expressed higher in Vessel B than Vessel A. ACTA2 had higher expression in the static condition than either of the BVMs. 


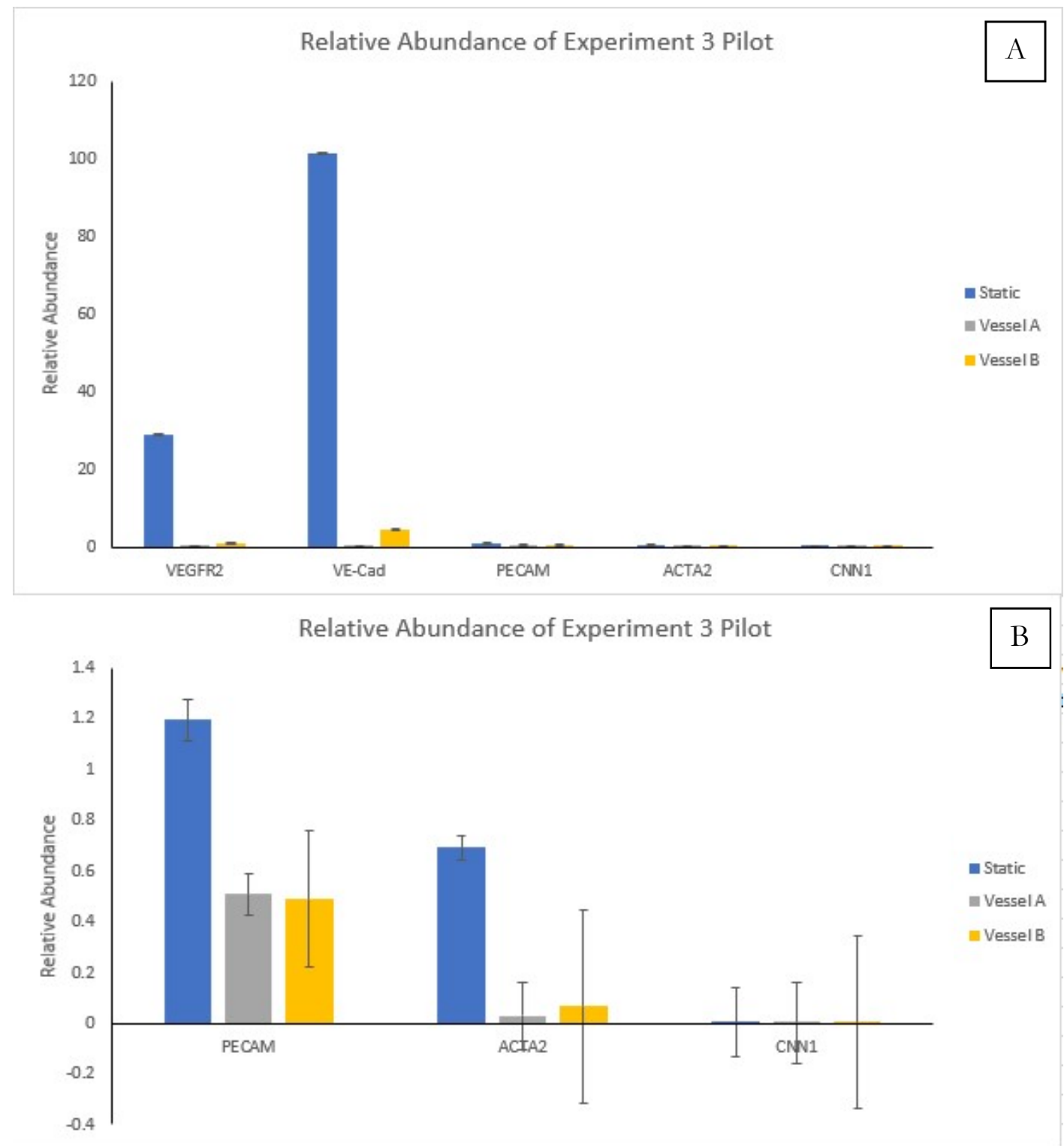

Figure 29: Experiment 3 Relative Abundance. HUVEC relative abundance of all GOI using GUS $\beta$ as the housekeeping gene. (A) all GOI and (B) PECAM, ACTA2, and CNN1.

Based on the results of this pilot, a full Experiment 3 was not explored. This study would have included more BVM replicates and multiple cell passage numbers. However, these three experiments did not indicate EndMT with the ECs during static culture or during culture in BVMs, which will be discussed in detail in the following section. 


\subsection{Discussion and Conclusion}

\subsubsection{Experiment 1 Pilot and Experiment 1}

The purpose of Aim 1 was to investigate and characterize the gene expression changes experienced by HUVECs in vitro with respect to increasing culture time and changing flow conditions during culture. The effect of cell culture time on HUVEC gene expression was established first through analyzing three endothelial markers and two mesenchymal markers. The primers for these markers were verified by calculating the efficiency of each primer assay. PECAM, VE-cad, ACTA2, and calponin had efficiency values between 90 and 100\%. Even though VEGFR2 and GUS $\beta$ had efficiency values of 88.57 and 86.5 , respectively, it was decided that these efficiency values were still acceptable, especially considering the primers were already preverified. Therefore, all six primers were concluded to be acceptable for use in qPCR experiments.

The pilot for Experiment 1 used HUVECs at passages 4, 6, 7, 8, 9, and 10 to study the effect of culture time on gene expression. The qPCR results were then analyzed using one of two housekeeping genes, $\beta \mathrm{ACT}$ or GUS $\beta$. The use of either housekeeper in analyzing relative abundance revealed similar trends. VE-cad had the highest abundance, followed by VEGFR2. Passage 4 HUVECs had significantly lower expression of VE-cad than passages 6 through 10 cells. Passages 6 and 7 had significantly lower expression than passages 8 through 10, creating a slight positive correlation between passage number and relative abundance. A slight positive correlation was also shown between passage number and VEGFR2 abundance. This trend is unexpected, as literature suggests that 
expression of endothelial markers like VE-cad and VEGFR2 decreases as passage number increases. However, the opposite trend was seen with this data.

The third EC marker, PECAM, did not have any expression differences between passage numbers when compared to $\beta \mathrm{ACT}$ or GUS $\beta$. Like with the other EC markers, it would be expected that PECAM abundance would decrease with increased culture time. PECAM abundance was also much lower than the other EC markers, and had an abundance closer to ACTA2. This observation was interesting, especially considering the expression of ACTA2 in HUVECs seen by immunostaining. The mesenchymal markers, ACTA2 and calponin, had no correlation between passage time and abundance. While literature suggests that these genes should increase with culture time, this was not observed.

Another important consideration of this pilot was the viability of $\beta \mathrm{ACT}$ and GUS $\beta$ as housekeepers. The $\mathrm{Ct}$ data for $\beta \mathrm{ACT}$ showed variability between passage time, making the gene non-ideal as a housekeeper. GUS $\beta$ expression, on the other hand, was stable over culture time. With this in mind, $\beta$ ACT was still included as a housekeeper in Experiment 1 to determine whether the inconsistent stability would be observed again.

Based on the results of the pilot, it was decided to expand the passage numbers used for Experiment 1; the culture times of the pilot may have not included early and late enough passages to elucidate all trends in gene expression. Therefore, passage times were increased to include passages 3, 5, 7, 9, and 11. The results of the $\mathrm{Ct}$ values for $\beta \mathrm{ACT}$ and GUS $\beta$ in Experiment 1 confirmed the results seen in the pilot. $\beta$ ACT was not stably expressed, while GUS $\beta$ expression remained relatively constant. Therefore, $\beta$ ACT was 
eliminated as a housekeeping gene, and GUS $\beta$ was used for data analysis in Experiment 1 and in all other experiments.

Experiment 1 result trends resembled those of the pilot. The later passages $(9,11$, 13) had higher expression of VE-cad than the early passages $(3,5,7)$. VEGFR2 expression increased with increased culture time. PECAM, ACTA2, and calponin expression did not change with increasing culture time. These data do not support the trends identified in literature, where HUVECs decrease expression of EC markers and increase expression of mesenchymal markers as EndMT occurs. In fact, this data suggests that the HUVECs are sustaining, and even forming, stronger cell-cell interactions as culture time increases. Two of the three mechanosensory complex markers, VE-cad and VEGFR2, increased abundance between early and late passages. The stability of PECAM expression with respect to culture time also supports the idea that culture time does not result in dedifferentiation.

The relationships seen in these data are difficult to explain. PECAM stability over culture time can be attributed to its function as a transmitter of mechanical force. Shear stress phosphorylates and activates PECAM; in the static culture condition, no shear forces was present to activate PECAM. PECAM RNA transcription should change as a result of mechanical force stimulation, so static culture should not increase PECAM expression. While this explanation seems plausible for PECAM, it contradicts VE-cad and VEGFR2 expression data. PECAM transmits mechanical force to VE-cad, which responds by modulating VEGFR2. Since no mechanical force is being transmitted by PECAM, VE-cad expression would be expected to remain constant. If VE-cad expression 
was constant, there should be no modulation of VEGFR2. However, both VE-cad and VEGFR2 were upregulated despite the lack of mechanical force.

One explanation for increased VE-cad expression could be the confluency of HUVECs used in the study. HUVECs were cultured to between 90 and $100 \%$ confluency, meaning high amounts of cell-cell interactions and establishment of contact integrity by VE-cad. However, by this explanation VEGFR2 expression would decrease, which was not the observed response. A simpler explanation is perhaps the result of a response initiated by VEGFR2 and not PECAM or VE-cad. HUVEC media contains VEGF, which binds VEGFR2 and causes downstream increases in VE-cad transcription. This explains the increase in expression over culture time, since an increase in culture time means more time spent in media containing VEGF. This pathway is also independent of PECAM, explaining the stability of PECAM expression over culture time. Regardless of any of these explanations, this endothelial marker data did not indicate any dedifferentiation occurring within the ECs.

The stability of the mesenchymal markers adds to the conclusion that culture time does not contribute to dedifferentiation, or at least suggests that a mesenchymal genetic change does not occur in the culture period tested here. Furthermore, the expression of mesenchymal markers in HUVECs was almost negligible when compared to the same markers in HUASMCs. It would not be expected that HUVECs would express those markers at an abundance even close to that of HUASMCs. However, one study showed that mesenchymal-progenitor cells produced between 2 and 9 times less ACTA2 and between 5 and 50 times less calponin than $\mathrm{SMC}^{119}$. EndMT would result in higher ACTA2 and calponin abundance than those found in this experiment. 
This experiment found that static culture time was a variable contributing to changes in gene expression. However, dedifferentiation towards a mesenchymal state was not observed over culture time. Since culture duration influences gene expression, several passage numbers were included in experiments which tested multiple flow conditions.

\subsubsection{Experiment 2}

With the effect of static culture time determined by Experiment 1, the second part of Aim 1 could be pursued: to investigate the effect of flow conditions on HUVEC gene expression. Experiment 2 served to establish a disturbed flow condition; this disturbed flow, as well as the static culture, was used for comparing the effect of laminar flow on HUVECs in the BVMs. The SMC media treatment group served as a positive control for gene expression change towards a mesenchymal state. GUS $\beta$ expression remained stable despite the addition of two new treatments, meaning that GUS $\beta$ retained its viability as a housekeeper.

The non-treatment, or static culture in HUVEC media, group resulted in similar trends as seen in Experiment 1. Gene expression in the SMC media treatment group trended in a direction that aligns with a mesenchymal shift; VE-cad and VEGFR2 expression decreased as passage number increased, and ACTA2 expression had an increasing trend with passage number. While PECAM expression was stable with passage number for the SMC media treatment group, expression was lower than that of the non-treatment group. These results confirmed that the treatment of HUVECs with SMC media served as a positive control for EndMT. It is important to note that SMC media treatment length was not a variable, as all passages of HUVECs were treated with 
SMC media for the same length of time; therefore, any trends in gene expression can be attributed to the culture duration variable.

Since a high enough concentration of RNA was only isolated from the disturbed flow treatment at passage 14, passage number did not serve as a variable in this case. However, the data collected at the singular passage number was still interesting. The disturbed flow HUVECs had significantly lower expression of VE-cad and VEGFR2 than all non-treatment group passages. The SMC media treated passage $10 \mathrm{HUVECs} \mathrm{had}$ similar expression to the passage 14 disturbed flow cells. Passage 6 SMC-media treated cells had higher expression of VE-cad than the disturbed flow cells, while the passage 14 cells had lower expression. The disturbed flow condition had lower expression of VEGFR2 compared to all SMC media treated passages. PECAM expression was not different between disturbed flow and SMC media treatments. Surprisingly, ACTA2 expression of the disturbed flow condition resembled non-treatment expression, which was lower than that of SMC media treatment. Taken together, these comparisons indicated that the SMC media-treated group succeeded as a positive control for EndMT while the disturbed flow condition achieved some loss in endothelial markers and some gain of mesenchymal markers.

PECAM expression in the disturbed flow group was not different than the nontreatment group, so the effects of disturbed flow on VE-cad and VEGFR2 expression worked independent of PECAM expression. It is not necessarily surprising that PECAM expression remained constant regardless of treatment; changes in PECAM expression are more heavily connected to cell-cell contact than to shear stress. Balaguru et al. found that PECAM expression remained stable between static and disturbed flow conditions ${ }^{120}$. 
Changes in VE-cad and VEGFR2 expression are more correlated with flow changes, which was observed by the results of this experiment. The downregulation of VE-cad and VEGFR2 compared to the non-treatment group demonstrated the utility of the disturbed flow condition in being an inducer of EndMT.

While EC marker expression indicated induction of EndMT by the disturbed flow protocol, it did not align with the expression of mesenchymal markers. The trends that would be expected from EndMT induction were observed by the positive control treatment of SMC media, supporting the treatment's use as a positive control for EndMT. This treatment also aligned well with the result of the disturbed flow condition, except with regards to ACTA2. This could simply be a limitation of the time point at which HUVECs were exposed to disturbed flow. Despite this, Experiment 2 succeeded in validating the method of inducing disturbed flow and establishing gene expression changes of a single passage of HUVECs exposed to this flow.

\subsubsection{Experiment 3 Pilot}

Experiment 3 was intended to combine previously investigated flow conditions with BVMs to provide an encompassing set of data on how gene expression changed between static culture, disturbed flow, and laminar flow as culture time increased. The pilot for this study eliminated the factor of culture time, and only investigated flow conditions at a single passage of HUVECs.

As with previous experiments, the stability of the reference gene GUS $\beta$ had to be determined. Upon plotting the Ct values, it appeared that GUS $\beta$ was not stable with the addition of new flow conditions. The two BVMs had Ct averages of 27.72 and 23.56 
cycles, which was higher than the $\mathrm{Ct}$ average of the static culture at 19.9 cycles. While the static culture maintained a $\mathrm{Ct}$ value similar to previous experiments, the disturbed flow condition did not. Experiment 2 resulted in a Ct average of 20.45 cycles, while this pilot resulted in a Ct value of 39.49 cycles. This discrepancy between $\mathrm{Ct}$ values for the disturbed flow condition between the two experiments was alarming. It indicated that the gene was barely expressed at all and took almost all the cycles for a signal to be detected. Since this was not observed in Experiment 2 for the same treatment condition, other factors were looked at to explain this. Only one of the three wells ran with this treatment resulted in a signal, indicating a potential issue with the either the RNA quality or cDNA concentration. The disturbed flow RNA had 260/280 and 260/230 ratios of 2.01 and 2.18, respectively. These values were within the accepted range for RNA purity, so the dissociation curves were analyzed to determine whether the reaction products caused an issue.

It was found that all GOI besides PECAM had multiple peaks in the disturbed flow condition, and therefore were omitted from analysis in this experiment only. Even though PECAM had a single peak in its dissociation curve, it was still omitted as an outlier because of the extremity of the resulting abundance value. There was most likely an issue with this individual PECAM sample, and the disturbed flow condition itself did not cause the high $\mathrm{Ct}$ value observed here. However, the exact cause of this was unclear. More repetitions of the flow condition are required to identify the source of this issue, or whether this issue will persist. The issues found by the dissociation curve with all other GOI in this group was also surprising, since the same issues were not found in a similar treatment group in Experiment 2. 
The BVM RNA values were also concerning. The 260/280 ratios were 1.63 and 1.81 and the $260 / 230$ ratios were 0.35 and 0.60 . While the $260 / 280$ ratios were only slightly below an ideal ratio, the 260/230 ratios were excessively low. A cause for both low 260/280 and 260/230 ratios is contaminating phenol from the isolation protocol ${ }^{121}$. Phenol is a main component of Trizol, and if the supernatant is not carefully separated during the isolation phenol could remain in the final product. This explanation is plausible, but unlikely considering the RNA purity of the static and disturbed flow samples were isolated using the same protocol and resulted in appropriate purity ratios. The BVMs, unlike the other two conditions, had scaffolds that were homogenized. It is unknown how the scaffolds affect the purity of the isolated RNA but could potentially cause contamination. The dissociation curve for each gene had a singular peak, shown in Figure 30, so the low RNA purity ratios may have not affected the quality of the PCR products.

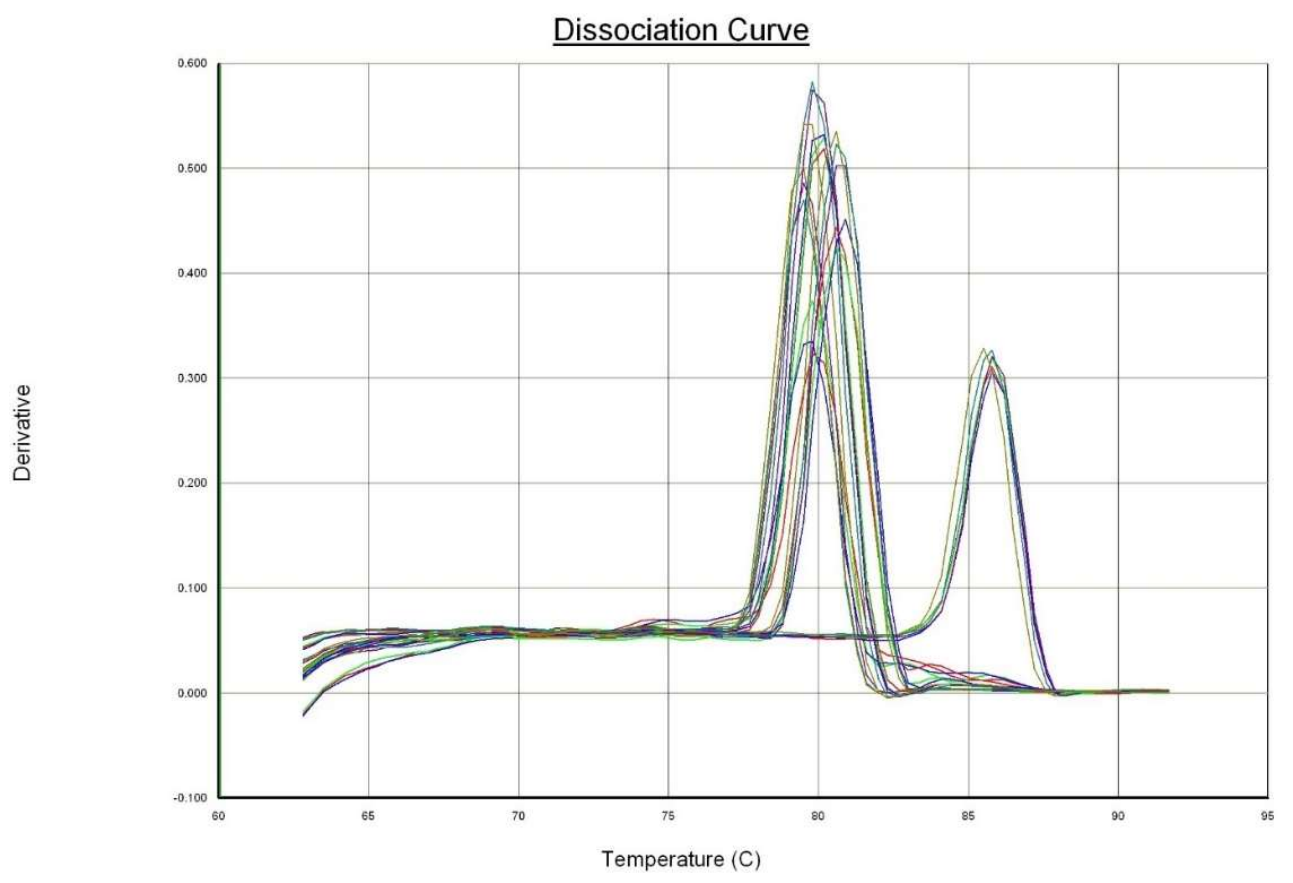

Figure 30: Dissociation Curves for GOI for EC-only BVMs. 
The results of this experiment were not expected. Shear stress has been shown to increase VE-cad and VEGFR2 expression in ECs compared to ECs in static culture ${ }^{92,122}$. The opposite was found in this experiment; VE-cad and VEGFR2 expression decreased relative to static culture. Two theories persist to explain these results. First, the amount of shear stress in the BVMs may be too low to induce EC activation. The shear stress in BVMs is approximately $0.35 \mathrm{dyne} / \mathrm{cm}^{2}$, which is significantly lower than shear stresses used in literature that showed upregulation of VE-cad and VEGFR2 which were between 10 and 20 dyne $/ \mathrm{cm}^{2}$.

A more likely explanation is that the 24-hour time point used for the BVMs was too short a duration to observe changes in EC expression. A study using a similar shear stress to the one in BVMs found that VE-cad was significantly downregulated 24 hours after flow induction ${ }^{123}$. VE-cad interactions must be broken to adapt to changes in stimulus, such as during the induction of flow. The studies that found an increase in VEcad and VEGFR2 expression used between 3 and 7 days of flow exposure. This explanation also gains merit from the expression of PECAM in this study. The previous experiments in this aim saw relatively stable expression of PECAM. However, this study saw lower expression of PECAM in the BVMs than in the static culture. This indicates that the cell-cell contact, which is necessary for PECAM expression, may have not been reestablished at this time point.

While it is disappointing that the disturbed flow condition was emitted, it may not have contributed to an explanation of these findings because of the short duration. Expression levels of mesenchymal markers were also not meaningful if the time point 
was too short. ACTA2 abundance was higher in static culture than in BVMs. It would be interesting to see if this trend persists at longer time point BVM, such as at 3 or 7 days.

The data collected by the pilot stemmed the decision to not pursue a full study. The experiments did not indicate EndMT occurring during culture or within the two BVMs of this pilot. The exploration of EndMT was the main topic of interest, and while the results gathered by these experiments were useful, they did not support any dedifferentiation occurring.

\subsubsection{Aim 1: Limitations and Future Work}

Several limitations became clear from these experiments. The stability of GUS $\beta$ in BVMs was a limitation, but this was worked around by averaging GUS $\beta$ Ct values in experiment 3. Another limitation was the quality of RNA from the BVMs. This could have been caused by the isolation protocol, but more likely was a result of introducing scaffolds in the RNA isolation. How, and why this would occur is unclear. However, the greatest limitation was not completing a full Experiment 3 study. Even if passage number was kept constant, a full experiment that included many replicates of vessels would have helped discern the consistency from vessel to vessel, both regarding genes of interest variability and housekeeping gene stability. It is disappointing to have established the methods and completed the prerequisite work for a full experiment to compare the three flow conditions, only to not complete the full study; however, this decision was logical. Since the results previously discussed did not indicate a shift towards a mesenchymal phenotype, it did not seem productive to continue this exploration. 
This area of the project still has areas that can be explored and improved. A full experiment could be conducted to compare the three flow conditions established here, not as a means to determine dedifferentiation but to better understand how the laminar flow in our model affects the cell-cell interactions. This experiment could be set up to study the effect of increasing RPMs on our model; increasing the flow rate would improve the physiological relevance of the model by increasing the shear stress. Shear stress would not be able to reach the conditions of the native vessel without changes to the viscosity of the media, but the methods explored here could be utilized for this exploration ${ }^{37}$. A different experiment could also include BVMs at different timepoints to verify if trends from longer timepoints agree with those from literature. A disturbed flow condition would be beneficial if that study was to be performed. Hui et al. found that VE-cad distribution was intermittent after exposure to disturbed flow, whereas a continuous distribution of VE-cad was observed after exposure to laminar flow ${ }^{124}$. This study could compare the effects of disturbed flow to laminar flow since the extent of mechanosensory complex reestablishment differs depending on flow type.

The methods used in these experiments could also be improved in the future. By using 6-well plates, two flow conditions are present within that flow treatment. The edges of the well plate are exposed to disturbed flow at a higher shear than the center. Well plates shaped like a ring are available, which eliminates the center of the well plate so the shear on all cells would be similar. More time points could be explored for the disturbed flow condition to observe how expression changes after multiple days of exposure to disturbed flow. This could be used to compare against 3, 7 or 14-day BVMs. 
These methods can also be used to generate data in support of other projects. One possible application is in the exploration of novel scaffolds to understand how the cellcell interaction changes between scaffold types. A scaffold that retains cells better within the lumen should have higher expression of the three mechanosensory complex markers used in the previous experiments. Specifically, this scaffold material would result in EConly BVMs with elevated expression of PECAM, VE-cad, and VEGFR2 because more cell-cell junctions exist. However, for the current thesis, five markers were used to asses EndMT for increasing cell passages and for static, disturbed, and laminar flow conditions through changes in gene expression. 


\section{CHAPTER 3. AIM 2: CHARACTERIZATION OF NOVEL SCAFFOLDS THROUGH HUVEC CASPASE ACTIVITY AND SCAFFOLD COVERAGE}

\subsection{Introduction}

After evaluating gene expression changes in Aim 1, Aim 2 sought to establish methods to better understand how cells and scaffolds interact in the BVM model. Methods for quantifying cytotoxicity, short-term cell adhesion, and long-term cell coverage were investigated. These methods were applied by comparing the current scaffolds with novel, nanofiber scaffolds. The following sections will overview how cells interact with and respond to scaffolds in tissue engineering applications. It will also introduce the novelty of adding BTEAC salt to scaffolds for nanofiber production.

\subsubsection{Scaffold Effects on Cells}

Tissue engineers attempt to create biomaterial scaffolds that resemble the native ECM of the tissue that is being mimicked. Electrospinning is a useful technique for creating scaffolds because scaffolds can be customized during fabrication by adjusting a variety of parameters, like voltage, percent polymer, polymer solution flow rate, and collector speed. Regardless of the fabrication process and material used, there are some general trends between scaffold characteristics and cell interactions. These scaffold characteristics include fiber diameter, fiber alignment, porosity, stiffness, topography, and scaffold degradation. Understanding the possible effects of these characteristics on cells is important when fabricating a scaffold using new solvents or additives. 


\subsubsection{Fiber Diameter}

Figure 31 demonstrates how fiber size affects cell attachment. Microfiber scaffolds encourage cell attachment to single fibers, which does not resemble a normal cellular interaction with native ECM. Nanofiber scaffolds encourage cells to form attachments with multiple fibers, which is closer to resembling native ECM where cells have many attachment $\operatorname{sites}^{125}$. As a result, fiber size effects cell morphology. This was demonstrated by Hodgkinson et al., where they found that primary human dermal fibroblasts became more elongated on microfiber scaffolds whereas cells on nanofiber scaffolds had a spread morphology ${ }^{126}$. These smaller fibers enhance cell attachment through increasing the available attachment sites, and the likelihood of these attachment forming is increased since the specific surface area of small fiber scaffolds is larger than that of scaffolds with larger fiber sizes. A higher surface area is also useful during the conditioning processes, as smaller fibers have increased protein adsorption ${ }^{127}$. As a result of increased protein adsorption and cell attachment, it is unsurprising that a decreased fiber diameter is associated with increased cell proliferation and viability ${ }^{126-128}$.
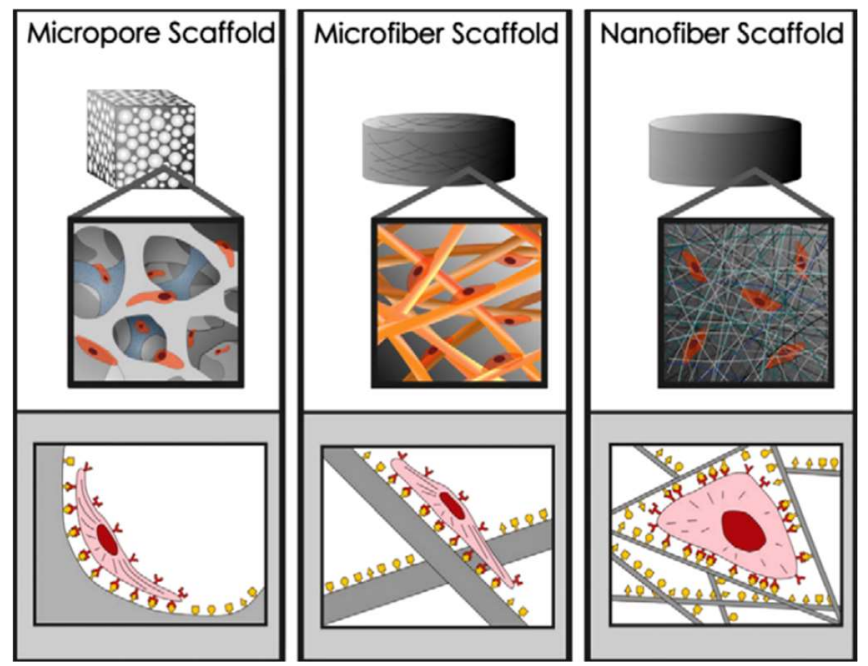

Figure 31: Cell Interactions with Fibers of Varying Sizes ${ }^{125}$. 


\subsubsection{Fiber Alignment}

Fiber orientation can be controlled during electrospinning but depends on the collector type and electrospinning parameters. The application of the scaffold dictates the benefits of aligned, semi-aligned, or random fibers. The Tissue Engineering Lab's PLGA scaffolds are fabricated such that the fiber orientation is random. However, it has been found that HUVECs adhere better to aligned fibers. Whited et al. found that aligned fibers led to increased HUVEC attachment to collagen-I/PCL scaffolds. Cells were seeded onto aligned, semi-aligned, or random fiber orientations and exposed to 20 dyne $/ \mathrm{cm}^{2}$ of shear stress. The aligned fiber scaffold had $95 \%$ cell adherence, while the semi-aligned and random fiber scaffolds had $80 \%$ and $60 \%$ adherence ${ }^{129}$. The aligned fibers in this study were oriented in the direction of flow, which is not possible to fabricate using the current electrospinning system in the Tissue Engineering Lab. Aligned fibers on a cylindrical mandrel would be perpendicular to flow, and it is unclear how this would affect adhesion.

\subsubsection{Porosity}

The electrospinning process results in a porous scaffolds, and random fibers have a higher porosity than aligned fibers ${ }^{127}$. Porosity mediates cell infiltration into scaffolds; if pores are too small cells cannot migrate into the scaffold, but if pores are too large the specific surface area is decreased and limits the area for cell attachment ${ }^{130,131}$. The migration or infiltration of cells in the scaffold is also dependent on the model or tissue being mimicked. For BVMs, a confluent monolayer of HUVECs should line the lumen, so cell infiltration would ideally be avoided. The fiber packing density is inversely 
correlated with porosity, where high fiber packing density results in low porosity ${ }^{132}$. Therefore, BVMs should have a high fiber packing density that encourages cells to proliferate along the lumen wall. For co-cultures of ECs and SMCs, like in the case of standard BVMs, it was found that pore diameters of $0.02,0.4,0.6$, and $0.8 \mu \mathrm{m}$ were optimal, whereas pore sizes greater than $2 \mu \mathrm{m}$ resulted in SMC migration into the scaffold $^{133}$.

Pore sizes can be divided into three categories: nano (>100 nm), micro (100 nm $100 \mu \mathrm{m})$, macro $(100 \mu \mathrm{m} \text { - millimeters })^{134}$. In vivo, macropores are useful for cell migration and vascularization, while micropores are effective for nutrient, oxygen, and chemical delivery ${ }^{135}$. Higher porosity in scaffolds is associated with greater cell deposition and adhesion. Murphy et al. used collagen-glycosaminoglycan scaffolds seeded with osteoblasts and found that the largest pore size resulted in the highest cell count at all time points. It was assumed that this was a result of cell migratory capacity, where at late time points small pores inhibited migration and proliferation ${ }^{130}$. The higher cell number could have also been a result of better cell adhesion due to increased protein deposition on the scaffolds, since scaffolds with higher porosity have been found to have higher protein adsorption than those with smaller pores ${ }^{135}$.

\subsubsection{Stiffness}

ECM stiffness can fluctuate drastically across tissue in vivo, as cells respond to the stiffness through changes in actomyosin interaction with the $\mathrm{ECM}^{136}$. A similar response with adhesion proteins occurs in vitro ${ }^{137}$. Stiffness is measured in terms of elastic modulus, where a stiff material has a high elastic modulus. Cell adhesion, 
spreading, proliferation, and even cell lineage is affected by scaffold elasticity, but how those characteristics are affected depend on the cell type ${ }^{132,138}$. For HUVECs, it was found that cells spread better and had a higher metabolic activity on stiffer surfaces $^{137,139}$. Stiffness also effects the shear level necessary to affect morphology. Stiffer surfaces required less shear stress to align in bovine aorta endothelial cells (BAECs) than soft surfaces. These stiffer surfaces also showed less of an inflammatory response $^{140}$.

\subsubsection{Topography}

The surface roughness of the PLGA scaffolds used in BVMs should be low upon removal from mandrels but may increase during ethanol sterilization and conditioning. Surface roughness can be divided into macroroughness (100 $\mu \mathrm{m}$ - millimeters), microroughness $(100 \mathrm{~nm}-100 \mu \mathrm{m})$, and nanoroughness (less than $100 \mathrm{~nm})^{132}$. Increasing surface microroughness is associated with an increase in cell adhesion and proliferation. Osteoblasts attached and proliferated most effectively on a surface with a roughness of $0.81 \mu \mathrm{m}$ than a smooth surface ${ }^{134}$. HUVECs showed increased cell adhesion and growth with increasing microroughness between $10 \mathrm{~nm}$ and $102 \mathrm{~nm}^{132}$. This trend could be a result of increased attachment sites on more rough surfaces.

\subsubsection{Scaffold Degradation}

PLGA degrades faster than either of its components, hydrophilic PGA and hydrophobic PLA, so PLGA composition percentages heavily effects degradation through changes in hydrophobicity. PLGA degradation occurs mainly as a result of ester 
bond hydrolysis, which forms acidic monomers than can reduce $\mathrm{pH}^{141}$. Degradation of PLGA in BVMs is reduced using 75:25 PLGA, while acidic byproducts are distributed throughout the system by dynamic flow so local $\mathrm{pH}$ around the scaffold does not increase. Despite this, scaffold degradation can have unintended byproducts that affect cell health and result in apoptosis.

\subsubsection{Measurement of Cell Responses}

Scaffold characteristics have diverse effects on cellular response, including apoptosis and adhesion. These are two of the most important cellular responses in relation to the BVM model and must be evaluated when proposing the use of a new scaffold. Assessment of apoptosis and adhesion will provide evidence for the benefits, or detriments, of introducing scaffold changes.

\subsubsection{Apoptosis}

Apoptosis is the mechanism by which cells are killed and removed by the body in a controlled procedure that includes membrane dissociation, cell shrinkage, condensation of chromatin, DNA fragmentation, engulfment, and removal ${ }^{142}$. In vivo, physiologic apoptosis occurs in natural and controlled processes, and is often associated with developmental biology where overproduction of cells is necessary to develop the nervous and immune systems. Pathologic apoptosis results from abnormal and uncontrolled levels of cell death, which is associated with cancer, AIDS, and Parkinson's disease ${ }^{143}$.

Apoptosis also occurs in vitro, except for events mediated by lymphocytes such as engulfment and removal. The inability to remove dead cells enhances cell apoptosis if 
cell debris is not cleared ${ }^{144}$. In the case of BVMs, apoptosis can occur through several factors. Scaffold degradation and improper $\mathrm{CO} 2$ conditions can lead to increased $\mathrm{pH}$ and apoptosis. Cell over-confluence or reduced cell adhesion also increases apoptosis. Therefore, induction of apoptosis must be understood.

\subsection{Apoptosis Pathways}

Apoptosis can be induced by either the extrinsic, intrinsic, or perforin/granzyme pathway, which converge on the execution pathway to end the cascade ${ }^{143}$. The extrinsic pathway initiates through tumor necrosis factor (TNF) transmembrane receptor-mediated interactions. These receptors transmit the signals through the membrane to initiate further intracellular signals. One common death ligand and receptor is Fas. The Fas ligand binds its receptor on the surface of the cells, which recruits Fas-associated protein with death domain (FADD) intracellularly. FADD associates and activates procaspase-8, activating caspase- 8 and the execution pathway ${ }^{145,146}$.

The intrinsic pathway is stimulated by positive or negative intracellular signals that cause changes to the mitochondrial membrane. Negative signals include the lack of growth factors, hormones, or cytokines. Positive signals include radiation, toxins, hypoxia, free radicals, and viral infections. These signals result in the opening of the mitochondrial permeability transition (MPT) pore that releases pro-apoptotic proteins, such as the intermembrane mitochondrial protein cytochrome $\mathrm{C}$. Cytochrome $\mathrm{C}$ binds Apaf-1 and procaspase- 9 upon being released into the cytosol, forming an apoptosome. Apaf- 1 contains a caspase recruitment domain, and the clustering of procaspase- 9 to cytochrome C and apaf-1 results in caspase-9 activation ${ }^{145,146}$. 
The perforin/granzyme pathway is activated by cytotoxic T lymphocytes (CTLs), which mainly targets tumor and virus-infected cells. CTLs secrete perforin, a poreforming transmembrane molecule, and then secrete cytoplasmic granules into the cell. Granzyme A and B are included in these granules; granzyme A activates DNA nicking, while granzyme B activates procaspase- $10^{145,146}$.

Caspase 8 in the extrinsic pathway, caspase 9 in the intrinsic pathway, and caspase 10 in the perforin/granzyme pathway all activate caspase-3, the initiator of the execution pathway. While caspase- 3 is the initiator, caspase- 6 and caspase 7 are also considered execution caspases. The three caspases activate cytoplasmic endonuclease to degrade nuclear material and proteases to degrade cytoskeletal proteins, ultimately resulting in cell death ${ }^{145,146}$.

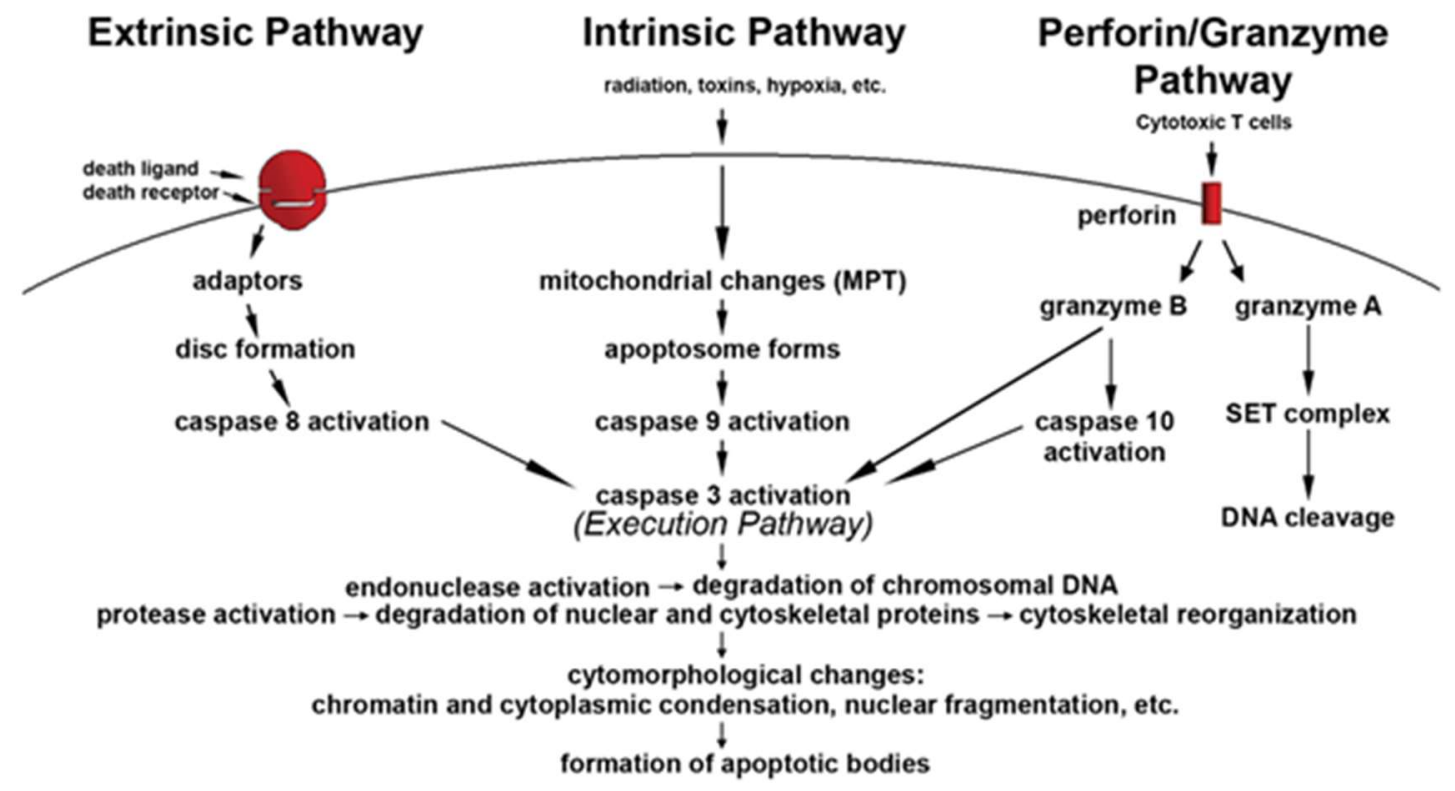

Figure 32: The Apoptosis Pathway. The three arms of the apoptosis pathways, converging on the execution pathway and cell death ${ }^{143}$. 


\subsection{Caspases}

Caspases, which stands for cysteine aspases, are heavily involved in the apoptosis cascade $^{147}$. There are at least 14 identified caspases, with 10 being major caspases. These include initiators (caspases $2,8,9,10$ ), proinflammatory cytokine activators (caspases 1 , $4,5$ ), and executioners (caspases $3,6,7)^{148}$. Caspases remain in an inactive proenzyme form until activation, referred to as procaspases ${ }^{143}$. Once active, caspases have two main functions: to breakdown proteins into smaller peptides and to activate other caspases to amplify the apoptotic signal. The proteins targeted include mediators and regulators of apoptosis, structural proteins, cellular DNA repair proteins, and cell cycle-related proteins $^{145}$. Since caspases are almost always associated with apoptosis, measurement of caspase activity can be used to assess apoptosis positive cells ${ }^{147}$.

\subsection{Apoptosis Detection and Quantification}

Methods exist to detect apoptosis in vitro, mostly through staining or collecting absorbance values. Toluidine and methylene blue are dyes that reveal intensely stained apoptotic cells. This method preserves the tissue or cells well but can underestimate apoptosis because it lacks the ability to detect smaller apoptotic proteins. Annexin $\mathrm{V}$ is a protein that binds phosphatidylserine present only on cell membrane surfaces of cells undergoing apoptosis. Annexins are proteins that bind phospholipids in the presence of calcium, which occurs on phosphatidylserine ${ }^{149}$. Since mitochondrial membrane potential changes occur early in apoptosis, cationic fluorescent dyes can be used to identify apoptotic cells. Healthy cells accumulate the dye in the mitochondria, while the stain remains in the cytosol of apoptotic cells ${ }^{150}$. 
Methods to label fragmented DNA are also used to detect apoptosis. DNA laddering through gel electrophoresis can be used in cases where high numbers of apoptotic cells are present. Since DNA is cut in relatively consistent sizes during apoptosis, regularly spaced bands can be identified on agarose gel. Terminal dUTP Nick End-Labeling (TUNEL) assays label DNA fragments with fluorescent nucleotides and use fluorescent intensity to identify apoptotic activity. However, this assay is known to falsely identify cells undergoing DNA repair or are active in gene transcription ${ }^{151}$.

As mentioned previously, caspase activity can be used to detect and quantify apoptosis, which can be achieved through fluorescent microscopy or measuring absorbance. One common caspase 3 assay, which indicates the initiation of the execution pathway, requires tissue homogenization to expose the active caspases ${ }^{151}$.

Immunostaining kits are also available which target the execution pathway, staining caspase 3 and 7, while others more broadly stain for caspase activity and label caspases 1 through $10^{152}$.

\subsubsection{Cell Adhesion}

\subsection{Passive In Vitro Cell Adhesion}

If endothelial cells are unable to form interactions with the surrounding scaffold within 24 to 48 hours after sodding, they will undergo apoptosis ${ }^{153}$. The adhesion process that occurs in culture flasks, petri dishes, and scaffolds of BVMs is known as static cell adhesion. This adhesion process differs greatly from the events that occur in vivo, where endothelium adhesion processes occur in dynamic conditions defined by tethering, rolling, adhesion, and transmigration ${ }^{154}$. In vitro adhesion can be divided into non- 
receptor and receptor-mediated binding. Non-receptor-mediated cell adhesion includes weak chemical bonds between cell membranes and substrates, such as electrostatic, polar, or ionic interactions. Receptor-mediated adhesion creates far stronger and specific interactions, in which interactions occur between matrix molecules and cell adhesion proteins $^{153}$.

Static adhesion has three main phases, shown in Figure 33: cell attachment, flattening and spreading, and reorganization of the cytoskeleton with the development of focal adhesion complexes (FAC). Integrin interactions with the substrate are responsible for the initial cell attachment, and the strength of the interaction increases over time as more receptor-ligand bonds are formed ${ }^{154}$. As more interactions form, cell spreading begins where the cell body decreases in height and increases contact area with the substrate. During spreading the actin cytoskeleton of the cell begins to reorganize. With this reorganization comes the formation of FAC, which connect integrins and other adhesion molecules to the cytoskeleton ${ }^{155}$.

\begin{tabular}{|c|c|c|c|}
\hline $\begin{array}{c}\text { Cell Adhesion } \\
\text { Phases }\end{array}$ & Phase I & Phase II & Phase III \\
\hline $\begin{array}{c}\text { Schematic diagram } \\
\text { of cell adhesion }\end{array}$ & 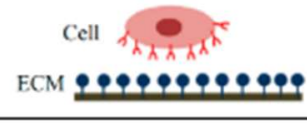 & Cell & ECM \\
\hline $\begin{array}{l}\text { Schematic diagram } \\
\text { of the transformation } \\
\text { of cell shape }\end{array}$ & Initial attachment & Flattening & $\begin{array}{l}\text { Fully spreading and structural } \\
\text { organization }\end{array}$ \\
\hline $\begin{array}{l}\text { Cell adhesion } \\
\text { intervension }\end{array}$ & $\begin{array}{c}\text { Electrostatic } \\
\text { interaction }\end{array}$ & Integrin bonding & Focal adhesion \\
\hline Adhesion stages & Sedimentation & Cell attachment & Cell spreading and stable adhesion \\
\hline
\end{tabular}

Figure 33: Cell Adhesion Phases. The progression of cell adhesion in vitro over three phases ${ }^{154}$. 
Fully developed focal adhesions are formed from over 150 proteins and researchers are still discovering exactly how these interactions develop; however, two kinases in particular are well researched and essential for FAC development ${ }^{156}$. Focal adhesion kinase (FAK) interacts with $\beta 1$ integrin cytoplasmic tails, as well as cytoskeletal proteins like paxillin. FAK is important for phosphorylation and assembly of focal adhesion components, where it functions mainly as a scaffold for other proteins to interact with. This function was determined through studies of kinase-dead FAK, where it was found that most of FAK's function was retained ${ }^{155}$.

Another essential kinase is Src, which is activated through its binding to FAK. While Src binds directly to $\beta$ integrins, the complex formed by FAK and Src plays important roles in cell spreading by relieving cytoskeletal tension during spreading and inhibiting cytoskeletal contractility ${ }^{156}$. These events are important for creation of stable focal adhesions and strong cell adhesion interactions.

\subsection{Cell Adhesion Tests}

Many tests exist to test cell adhesion to polymer surfaces, which can be divided broadly into tests for strength of adhesion and tests for percent adhesion. Some strength of adhesion test methods, like polyacrylamide-traction force microscopy (PA-TFM) and biomembrane force probes (BFPs), study single cell events. In PA-TFM, fluorescent beads are embedded near the surface of a polyacrylamide gel. When cell adhesion occurs, traction forces on the gel displace the beads so that bead movement can be quantified and related to adhesion force ${ }^{157}$. BFPs use a force transducer composed of two micropipettes, one holding a cell and the other holding an antibody-coated bead attached to the cell. 
Adhesion forces are quantified by cell displacement as a pulling force is exerted between the cell and bead ${ }^{158}$. Other tests for adhesion strength quantify the cell response through detachment. Centrifugation assays measure the number of cells that detach during centrifugation, which is reflective of cell adhesion strength ${ }^{154}$.

Tests for the percent of cell adhesion require knowledge of the initial number of cells seeded to a surface and are limited to short time points so that cell proliferation does not occur. Wash assays estimate cells remaining on a surface through counting removed cells. After performing wash steps on a surface with seeded cells, the cells from the wash solution are counted and subtracted from the known number of cells seeded to the surface ${ }^{154}$. Cell counting techniques have also been implemented that involve immunostaining and counting cells on a small fraction of the polymer surface, which can then be used to estimate cell adhesion density. These tests can be useful for determining cell adhesion potential, such as when investigating cell interactions with a novel scaffold.

\subsubsection{Addition of BTEAC Salt in Electrospinning of Scaffolds}

It has already been established that decreasing fiber diameter and porosity will increase EC adhesion to polymer scaffolds, making fiber diameter and porosity reduction a plausible solution to successfully creating BVMs that only use ECs. Therefore, methods for decreasing these scaffold characteristics were pursued in our laboratory by a different student researcher. One method investigated was to add benzyl triethyl ammonium chloride (BTEAC) salt to the PLGA and chloroform mixture before electrospinning. Figure 34 and Figure 35 compare standard electrospun scaffold versus a scaffold spun with 3\% BTEAC in the Tissue Engineering Lab (unpublished data). The average fiber 
diameter of the standard PLGA scaffolds was $5.55 \mu \mathrm{m}$, while the 3\% BTEAC scaffold had significantly smaller fibers with an average of $0.826 \mu \mathrm{m}(\mathrm{p}<0.01)$.

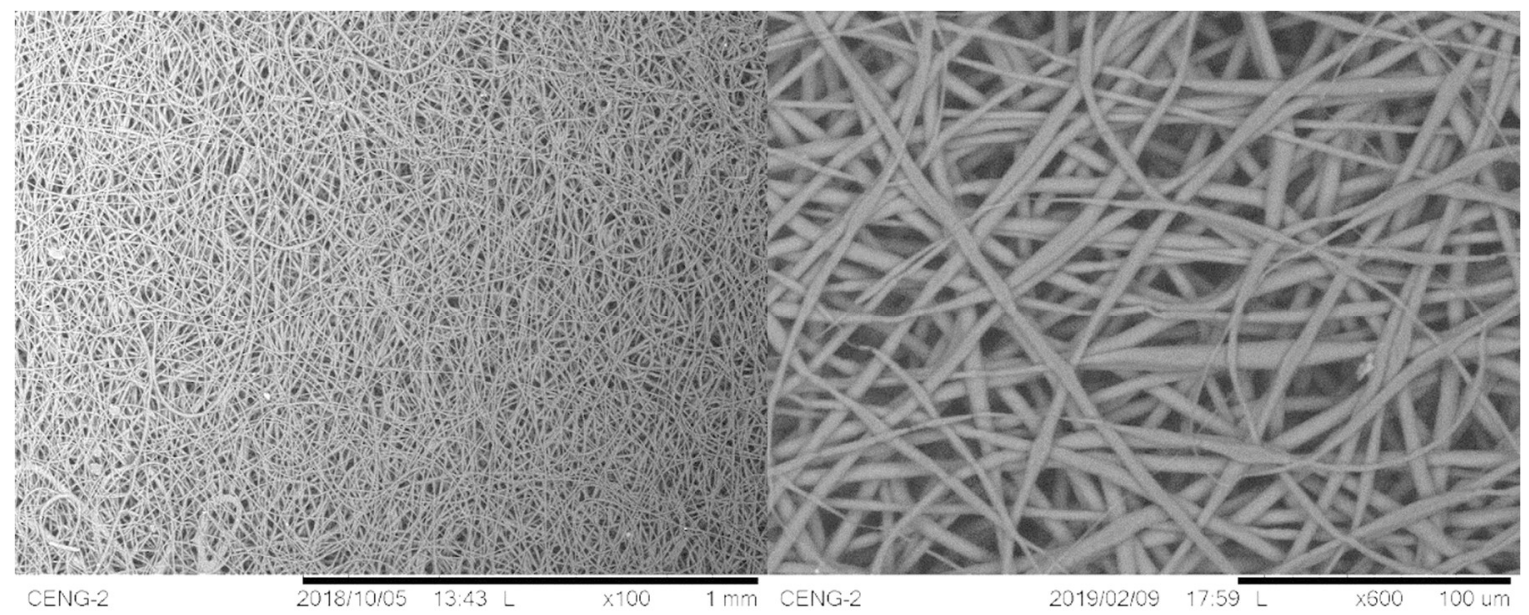

Figure 34: SEM of Standard Scaffold. Standard scaffold SEM images at 100x (left) and 600x (right) magnification.

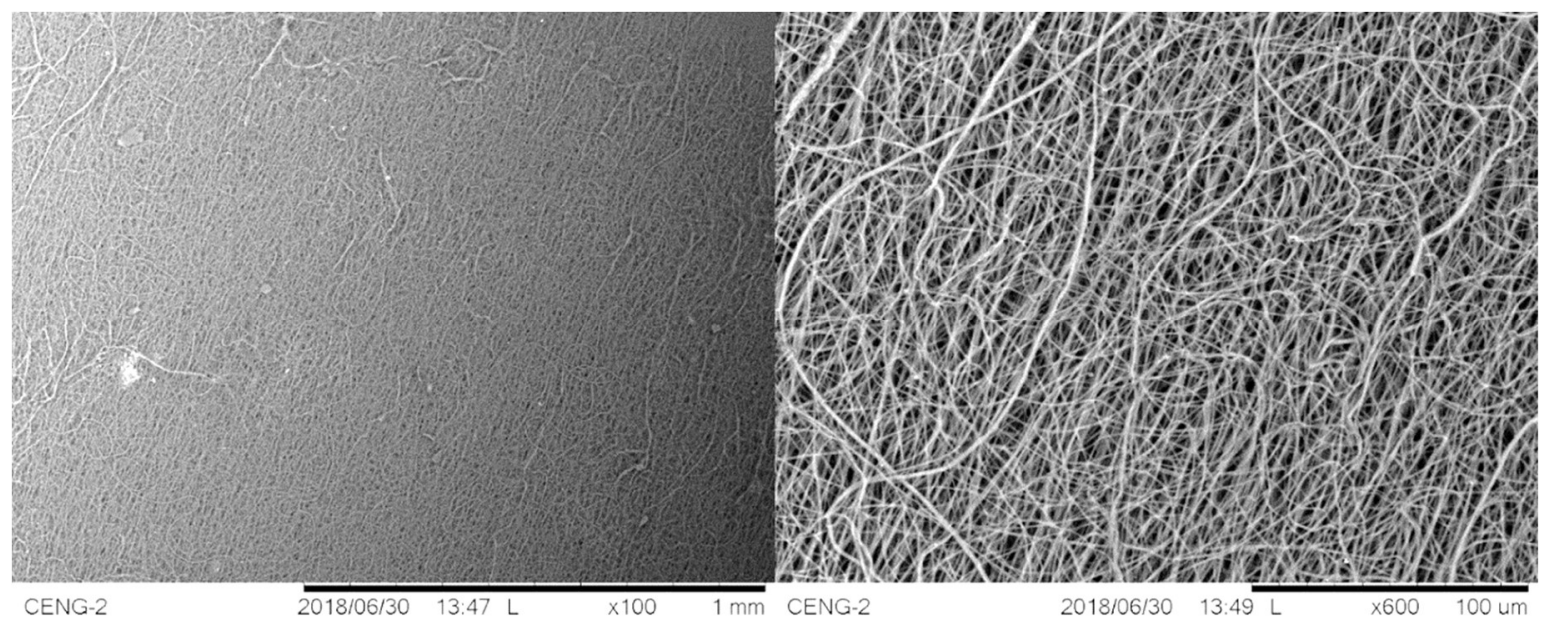

Figure 35: SEM of Salt Scaffold. 3\% benzyl triethyl ammonium chloride (BTEAC) salt scaffold SEM images at 100x (left) and 600x (right) magnification.

The addition of BTEAC has been shown to decrease fiber diameters of electrospun scaffolds ${ }^{159-161}$. The addition of the ionic salts like BTEAC increases the electrical conductivity of the solution by increasing the charge density. Within the electric field, the salt ions electrostatically repel each other, increasing the stretch of the 
polymer jet and reducing fiber diameter ${ }^{162}$. You et al. showed decreasing fiber diameter with increasing BTEAC content in PLGA scaffolds using chloroform as the solvent, seen in Figure $36^{160}$. Fiber diameter reduction is limited at salt concentrations above 3\% w/v. The same study also saw a decrease in porosity from $88 \%$ to $77.2 \%$. Another study which electrospun poly(hydroxybutrate-co-hydroxyvalerate) (PHBV) dissolved in chloroform saw a reduction in fiber diameter from $1662 \pm 490 \mathrm{~nm}$ to $603 \pm 250 \mathrm{~nm}$ after the addition of BTEAC ${ }^{163}$.

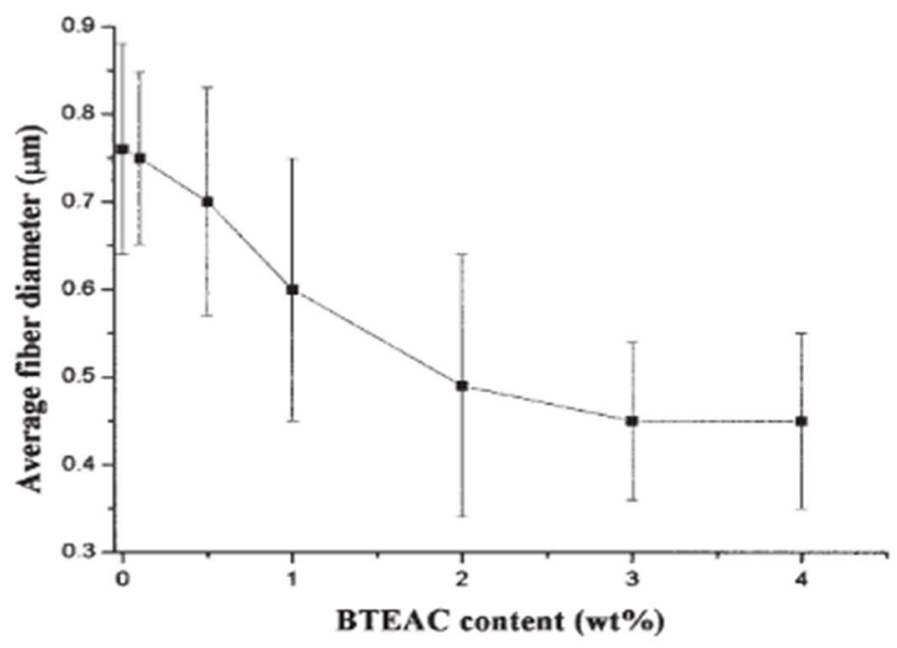

Figure 36: Fiber Diameter Reduction with BTEAC Percentage. The average fiber diameters of electrospun PLGA with varying percentages of BTEAC ${ }^{160}$.

The addition of BTEAC does not affect other important characteristics of the electrospinning solution, such as viscosity and surface tension, making it simple to implement in existing protocols ${ }^{159,160}$. Thorsten et al. electrospun PLA dissolved in either chloroform or dichloromethane and showed that, while electrical conductivity increases as BTEAC weight percentage increases, the surface tension of the solution remains constant $^{159}$. While the solution characteristics remain constant, the addition of BTEAC affects the mechanical strength of the resulting scaffolds. Scaffolds spun with BTEAC 
had a decreased ultimate tensile strength and Young's modulus as compared to those spun without the salt, but scaffolds with BTEAC had increased elongation at fracture ${ }^{163}$.

Interestingly, electrospun scaffolds with BTEAC have been investigated for antimicrobial properties for use as filters. Quaternary ammonium compounds (QACs), such as BTEAC, target and disrupt the cytoplasmic membrane of bacteria and the viral envelopes of viruses ${ }^{161}$. Park et al. characterized the antibacterial and antiviral effects of electrospun polyvinyl alcohol (PVA) with BTEAC dissolved in water. They found that the growth of K. pneumonia, E. coli, and S. aureus were inhibited at BTEAC concentrations above $2.6 \%$. Besides inhibiting growth, the scaffolds also reduced live bacteria and viruses over time. There was reduction of S. aureus and K. pneumoniae from 30 to 120 minutes, as well as reduction of PhiX174 and MS2 from 60 to 300 minutes ${ }^{161}$. These properties that make the BTEAC a good antimicrobial agent may disqualify it as a candidate for use in biomaterial scaffolds. If the salt has toxic interactions with cells, like it does with bacteria and viruses, cell death would increase while proliferation decreases. However, no current published studies have investigated the interaction of these scaffolds with human cells. Park et al. investigated BTEAC toxicity through leaching scaffolds and observing the effect of the leaching product on daphnia magna, a planktonic crustacean. They found that D. magna was not affected by BTEAC concentration between $0.1 \mathrm{mg} / \mathrm{L}$ and $5 \mathrm{mg} / \mathrm{L}$. The highest concentration leached from the scaffolds was $0.72 \mathrm{mg} / \mathrm{L}$, far less than a concentration that would be toxic to D. magna ${ }^{164}$.

While the addition of BTEAC salt to electrospinning solutions is a simple method to create nanofibers in scaffolds, there is potential for toxicity when cells interact with these fibers. For example, BTEAC salts have been shown to elute from the scaffolds in 
small amounts, but the effect of these salts has not been tested on cells ${ }^{164}$. Therefore, investigation of the cell interactions is necessary to determine the viability of these scaffolds for tissue engineering applications.

\subsubsection{Summary}

The prospect of implementing a new scaffold for use in BVMs requires an understanding of the interaction between cells and scaffold. This section outlines the effect of scaffold characteristics on cells, cell adhesion in vitro, and the apoptosis pathway. The following sections will describe how this knowledge was applied to create assays for testing scaffold cytotoxicity and cell adhesion along with experiments performed to verify the utility of these assays.

\subsection{Materials and Methods}

In order to implement a novel scaffold in the BVM model, three different assays were developed. The following sections will describe methods establishing these assays to compare BTEAC salt scaffolds against standard PLGA scaffolds. A large portion of developing these methods involved quantifying images that were collected through immunostaining and confocal imaging. The cytotoxicity elution assay tested scaffold viability indirectly, while the short-term adhesion and BVM cultivation assays determined direct utility of scaffolds on HUVECs. These direct toxicity assays provided useful data on HUVEC adhesion to and coverage on different scaffold types at increasing time intervals. 


\subsubsection{Caspase Stain Implementation and Verification}

Caspases are enzymes that are activated throughout the apoptosis cascades, so caspase activity is useful for assessing apoptosis. The Image-iT LIVE Green Poly Caspases Detection Kit (Molecular Probes) was used for immunostaining cells to visualize apoptotic activity. The kit also includes a Hoechst 33342 stain, which stains cell nuclei, and a Propidium Iodide stain, which stains dead cells. Besides the three stains, an Apoptosis wash buffer and Apoptosis fixative solution were included. The fixative solution was a $10 \%$ formaldehyde solution.

It was first necessary to establish positive controls to verify if the kit was performing properly; in this case, the positive control was a treatment that induced apoptosis. The caspase activity in this treatment serves as a baseline to compare the caspase activity of treatments in which toxicity is unknown. Hydrogen peroxide $\left(\mathrm{H}_{2} \mathrm{O}_{2}\right)$ and dimethylsulfoxide (DMSO) are known to be cytotoxic to cells, so these were chosen

as candidates for the positive control treatments ${ }^{165}$. HUVECs were grown to confluency in 8 -well plates before receiving one of the following 16 treatments: $2 \%, 5 \%, 10 \%$, or $15 \%$ DMSO in EC media for 30 minutes or 60 minutes, or $0.05 \%, 0.1 \%, 0.5 \%$, or $1 \%$ $\mathrm{H}_{2} \mathrm{O}_{2}$ in $\mathrm{EC}$ media for 2 minutes or 5 minutes. A negative control that received EC media, and no DMSO or $\mathrm{H}_{2} \mathrm{O}_{2}$, was also included.

After treatment, each well was washed with EC media before staining with the caspase kit. Cells were incubated for 90 minutes with $200 \mu \mathrm{L}$ of caspase detection reagent diluted in EC media. The staining solution was removed, and cells were washed with EC media before separately staining with Hoechst and Propidium Iodide, both at dilutions of 1:500 in EC media for 10 minutes. The cells were then washed twice with 
Apoptosis wash buffer before being fixed with Apoptosis fixative solution for at least 12 hours at room temperature before imaging. All samples in this aim were stained using this protocol; therefore, other sections will simply refer to this as "apoptosis staining protocol". This protocol is provided in Appendix J. Samples were imaged on the Confocal Microscope at a 40x magnification and the laser settings as seen in Table 4. Images were analyzed qualitatively based on caspase signal intensity. Samples with a strong caspase signal in the cytosol and nucleus but with low Propidium Iodide activity were selected as positive control treatments.

Table 4: Confocal Microscope Lasers and Settings.

\begin{tabular}{c|ccc}
\hline & Hoechst & Caspase & Propidium Iodide \\
\hline Laser & 405 & 488 & 559 \\
Laser Power & $15 \%$ & $30 \%$ & $30 \%$ \\
High Voltage & 700 & 700 & 700
\end{tabular}

Once positive control treatments were selected, each of the three individual stains needed to be checked for bleed-through to ensure that signal from one stain was not being detected by a separate laser. HUVECs cultured in 8-well plates were stained with either no stain, Hoechst stain only, Caspase stain only, Propidium Iodide stain only, or all three stains. These samples were imaged at 20x magnification and analyzed to determine if any bleed-through occurred. With these stains verified and positive controls established, the stain was then used with confidence to assess treatments in the following assays. 


\subsubsection{Cytotoxicity Assay}

The cytotoxicity of scaffolds was first assessed using a standard elution approach. This means that the assay was performed without direct cell-scaffold contact, but rather by treating cells with media that had been eluted with a scaffold. An elution is a liquid that has been incubated with a substance at a certain temperature for a length of time. The theory behind this is that any toxic particles will be drawn into the solution and the effect of this elution on cells will indicate the toxic effects of that original eluted substance ${ }^{166}$.

In this assay, standard PLGA scaffolds and 3\% BTEAC salt scaffolds were eluted in EC media for 72 hours at $50^{\circ} \mathrm{C}$ as advised by ISO-10993. To prepare the elution, scaffolds were cut to $43 \mathrm{~mm}$ and attached to fittings before being sterilized in $70 \%$ ethanol for 20 minutes. Using sterile technique, the scaffolds were then removed from the ethanol and flushed transluminally and transmurally with DPBS and EC media. The scaffolds were then placed in $10 \mathrm{~mL}$ of EC media and placed in a water bath heated to $50^{\circ} \mathrm{C}$. After 72 hours, the scaffolds were removed from the elution and stored at $4^{\circ} \mathrm{C}$ until use, approximately five to seven days. $\mathrm{pH}$ measurements were also taken of each elution to determine if heat or scaffold degradation had changed the media to be more acidic or basic.

The elution was next applied to HUVECs grown to approximately $80 \%$ confluence in 8-well plates. Cells were either treated with pure eluted media or eluted media that had been diluted by $50 \%$ for 24 or 48 hours. The dilution group was included because the strength of the original elution was unknown. If the full elution treatment was too cytotoxic there would be no way to discern between treatments. The dilution should emphasize any difference between the salt scaffold and standard scaffold elution, if a 
difference exists. After the treatment lengths, cells were stained using the apoptosis staining protocol and imaged at 40x magnification. Positive and negative controls were also included in each time group, where a positive control was treated with hydrogen peroxide just before staining and a negative control was treated with standard EC media. Image quantification methods had to be established to determine how many cells from each treatment group were positive for caspase or propidium iodide staining. To do this, images were separated by channel and false colored to the respective laser color (blue, green, or red). Nuclei were counted automatically using the Analyze Particle function in ImageJ, which required a maximum pixel area to be set. Therefore, any area below that set value would be excluded in the count, which prevented non-nuclei from being counted. Before counting, a threshold was set, and the image was made binary. An example of this process can be seen in Figure 37. Because of the variability in manually setting the threshold and the possibility of setting the size restriction too high, these settings needed to be verified.

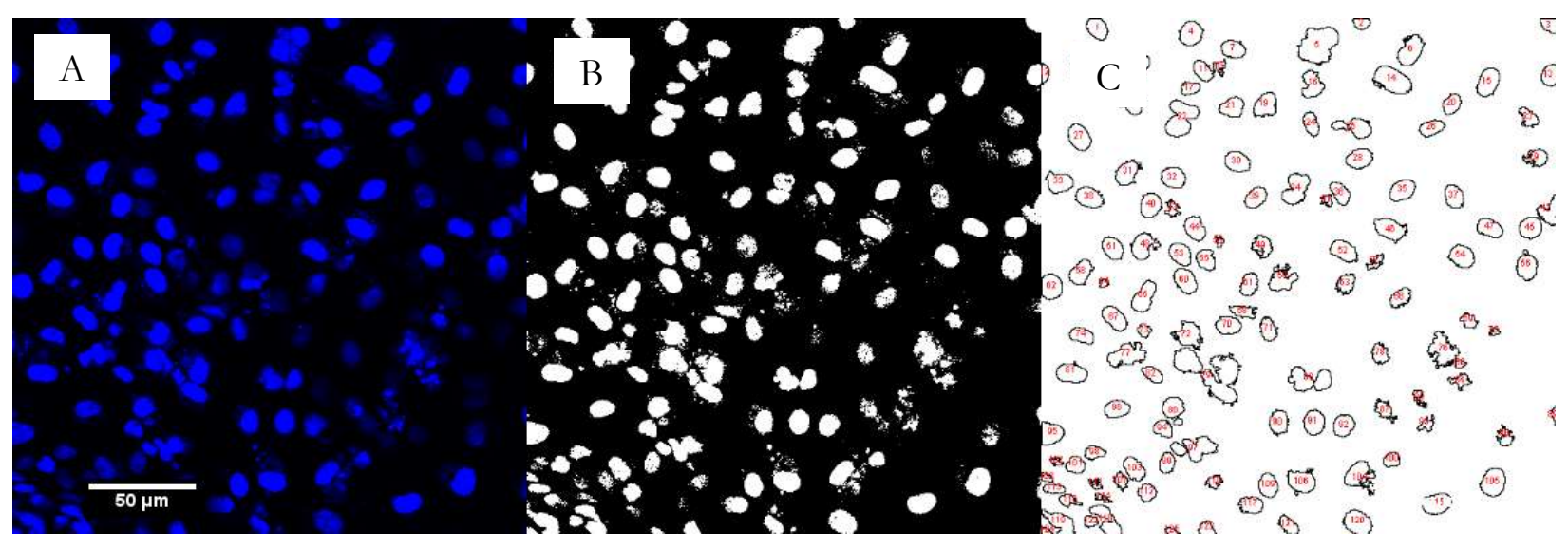

Figure 37: Hoechst Channel Automatic Cell Counting. (A) Raw Hoechst channel image with cell nuclei. (B) Threshold applied, reducing noise and making the image binary. (C) Outlines of automated cell count. 
The settings were tested by manually counting a sample of images and comparing the manual count to the automated count. The threshold values used varied but were set between 1000 and 1200, depending on the image and necessity to eliminate signals that were non-nuclei. Two magnifications were tested, 20x and 40x, and the smallest area excluded was set to 20 pixels $^{2}$ and 50 pixels $^{2}$ for the two magnifications, respectively. For the 20x magnification, it was found that there was an average error of $40.03 \%$ between the manual and automated counts with an average error of 18.5 nuclei per image. For the 40x magnification, there was an average error of $9.15 \%$ with an average error of 5.2 nuclei. With this data, it was clear that the 40x magnification was superior for analyzing. After automatically counting the Hoechst channel, the Caspase and Propidium Iodide channels needed to be quantified. These channels had to be counted manually because of the variability in signal location; some cells were positive for the stains in only the nucleus, others only in he cytoplasm, while some had s gnal throughout the entire cell. There was also overliap between cells, so automated counting would not be able to discern between large groups of cells. To aid with manual counting, pixel intensities below a certain value were eliminated to ensure that background noise did not appear to be a signal indicating the cells were positive for apoptc sis or cell death. This pixel reduction value had to be set to avoid eliminating cells that were truly positive for the stain versus those that were noise. Examples of these methods can be seen in Figure 38 and Figure 39. 


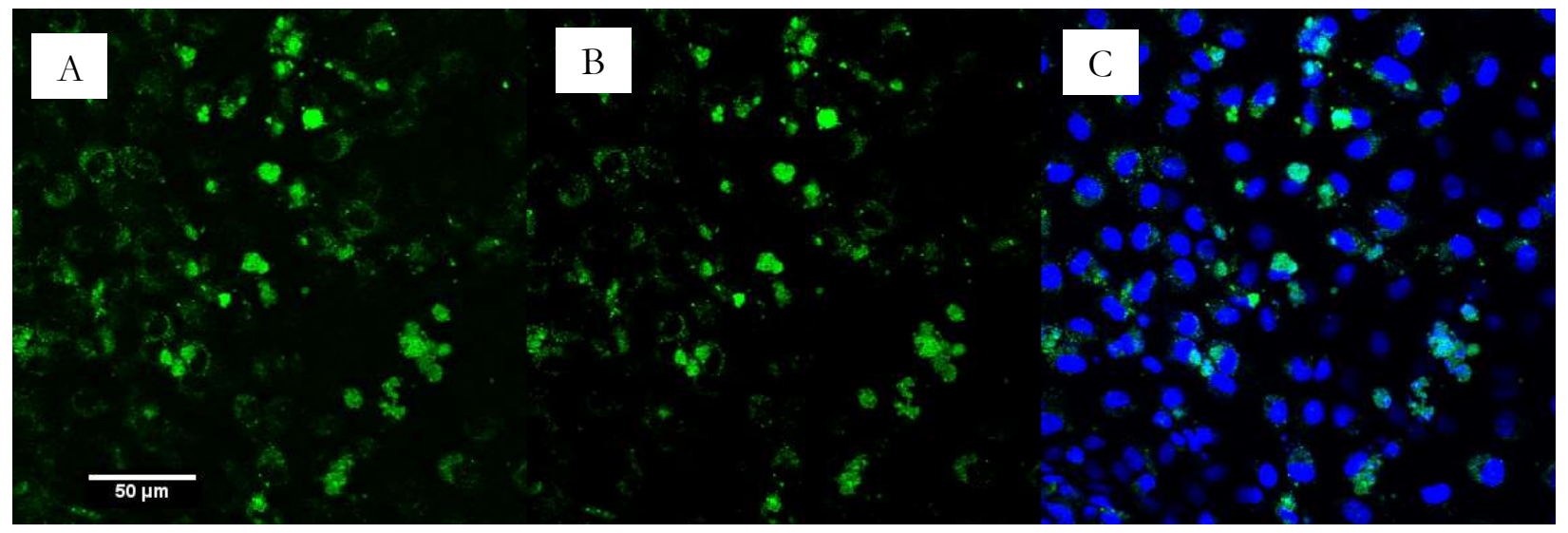

Figure 38: Pixel Reduction in Caspase Channel. (A) Raw image of Caspase channel. (B) Caspase channel after pixel reduction. (C) Overlay of Hoechst and Caspase channels, which was used for counting Caspase positive cells.
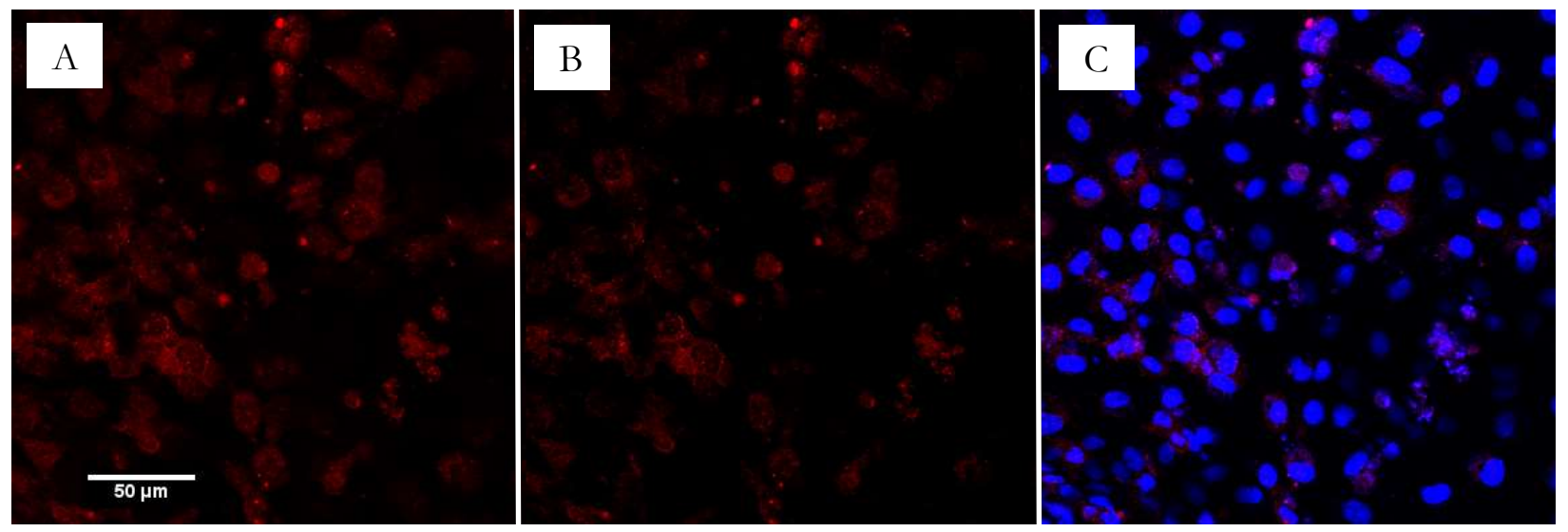

Figure 39: Pixel Reduction in Propidium Iodide Channel. (A) Raw image of Propidium Iodide channel. (B) Propidium Iodide channel after pixel reduction. (C) Overlay of Hoechst and Propidium Iodide channels, which was used for counting Propidium Iodide positive cells.

Graphs were created for Caspase and Propidium Iodide channels of pixel

reduction values versus number of cells manually counted. Pixel intensity values range from 0 (black) to 255 (white), so the values tested were in the lower range of intensities. For both stains cell count started to decrease rapidly after eliminating pixels with an intensity over 15, so this was set as the pixel elimination quantity for the Caspase and 
Propidium Iodide images. After eliminating these pixels, the Hoechst channel was overlaid on both channels separately. Only signals that surrounded or overlaid with nuclei were counted, so signals not associated with a nucleus were not counted as cells. After quantification, the percent Caspase positive cells and percent Propidium Iodide cells were calculated by comparing the number of positive stained cells to the total number of nuclei. Finally, the percent positive cells for both caspase and propidium iodide were normalized to the negative control (non-treatment) group, which were cells with only EC media, essentially subtracting out the natural population of positive cells for both stains that were present in groups that did not receive an elution treatment.

\subsubsection{Cell Adhesion and Viability Assay}

The nanofibers of the BTEAC salt scaffolds have much smaller fiber diameters than the standard scaffolds, which should improve EC adhesion ${ }^{167}$. Therefore, it was valuable to investigate cell adhesion to the scaffold at a short time point. This adhesion assay involved pressure sodding ECs to both types of scaffolds and analyzing the scaffolds after 4 hours. Methods were also determined to quantify cell adhesion and short-term data on Caspase activity and number of dead cells.

Since this time point was short, the assay could be conducted without using sterile technique. Standard scaffolds and 3\% BTEAC salt scaffolds were attached to fittings and sterilized in $70 \%$ ethanol for 20 minutes. The scaffolds were then flushed transluminally and transmurally in DPBS and EC media. HUVECs were then pressure sodded into the scaffolds by placing a stop-cap on one end of the scaffold, and then using a syringe to push cells resuspended in media into the scaffold. The cell density of the resuspended 
cells was counted, and the volume of cell solution sodded for each scaffold was recorded. The cell-scaffold construct was then incubated at $37^{\circ} \mathrm{C}$ for 4 hours in a petri dish filled with EC media. After 4 hours, constructs were removed and stained using the apoptosis staining protocol. The media that the constructs were incubated in was collected and spun down, and the cells that had adhered to the petri dish were also trypsinized and spun down. These cells were counted to determine how many cells did not adhere to the scaffold from the volume that was sodded.

Image quantification methods had to be established to determine cell coverage, caspase activity, and dead cell coverage from the images taken on the confocal microscope. Images were taken at 20x magnification, as the focal plane of the microscope does not reach the curved scaffold at 40x magnification. Due to this, individual cell nuclei could not accurately be counted. Instead, an assumption regarding the size of a cell nuclei compared to the whole area had to be made to estimate the number of cells present. Using sample 20x magnification images from literature of HUVECs stained with Hoechst and cadherin (which outlines the cytosol), the ratio of nucleus size compared to the entire cell size was estimated to be $23.42 \%$. Dividing $100 \%$ (the area of the entire cell) by $23.42 \%$ gives a value of 4.27 , which acts as a multiplication factor for the total stained nuclei area to extrapolate a cell coverage estimation. The Analyze Particle function in ImageJ was used to calculate the total nuclei area of each image in pixels ${ }^{2}$, seen in Figure 40, which was then multiplied by 4.27 to estimate cell area. The cell area estimation was then compared to the whole image area to determine percent of cell coverage. 


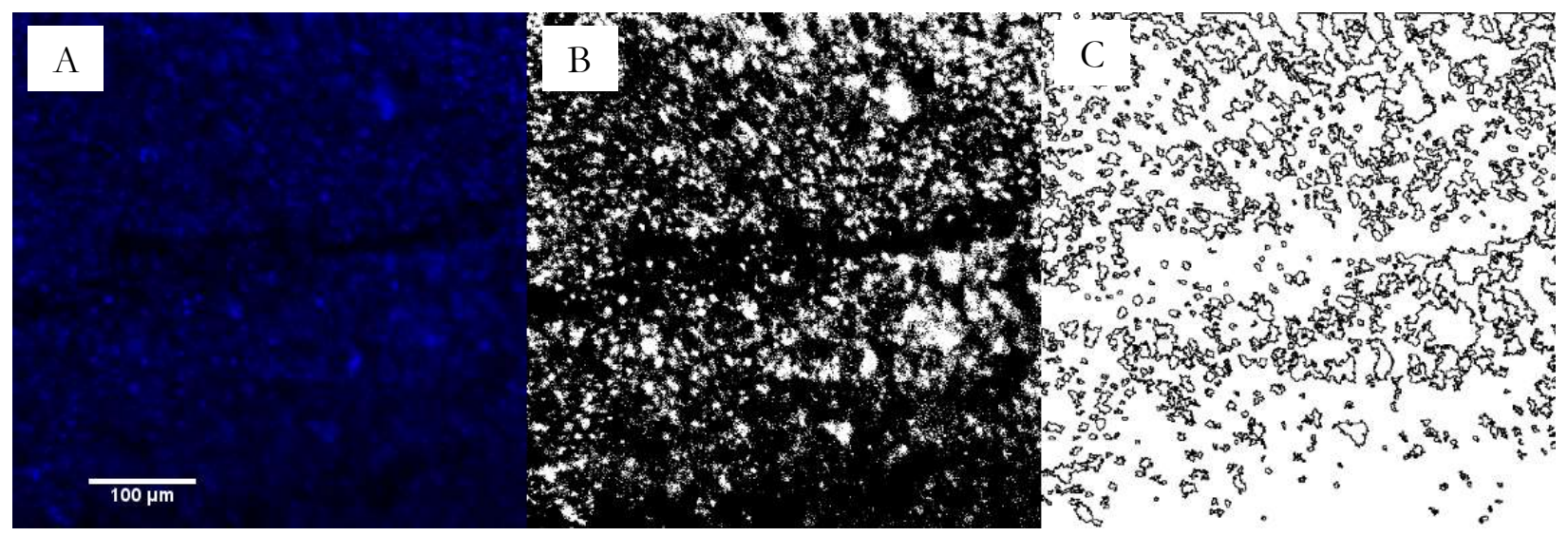

Figure 40: Hoechst Channel Coverage Quantification. (A) Raw Hoechst image. (B) Hoechst channel with threshold applied. (C) Automated cell area quantification.

The Caspase and Propidium Iodide channel images were quantified in a similar method, although the assumption was made that the whole cell was fluorescing in these channels, so no multiplication factor was necessary to determine signal coverage. The Analyze Particle function was used again to determine area of both Caspase and Propidium Iodide activity, and a percent coverage was calculated. This coverage was then normalized to the Hoechst channel cell coverage percentage of the same image to determine an estimated percent apoptotic or dead cells. Salt scaffold coverage in all three channels was compared against coverage of the standard scaffolds.

\subsubsection{BVM Cell Coverage and Viability Assay}

The final assay developed in this aim compared the standard scaffolds against two types of BTEAC scaffolds in BVM constructs at 3 and 7-day time points. Seven vessels were included in each time point: two standard PLGA scaffolds, two 0.5\% BTEAC salt scaffolds, two 3\% BTEAC salt scaffolds, and one ePTFE scaffold. The $0.5 \%$ BTEAC salt scaffold served as a low-salt group, while the 3\% BTEAC salt was a high-salt group. The 
ePTFE served as a negative control because ePTFE is known to be bioinert and nontoxic to cells. Since the standard PLGA scaffolds have not been characterized before, they could not serve as a negative control.

The standard methods for constructing EC-only BVMs were used, which can be found in Appendix H and Appendix I. One additional step had to be used on the salt scaffolds; it had been found that these scaffolds shrink substantially upon ethanol sterilization, both in length and circumferentially. Therefore, $4 \mathrm{~mm}$ salt scaffolds were electrospun and were then pre-shrunk on $3 \mathrm{~mm}$ mandrels in $70 \%$ ethanol for 15 minutes before being cut to the appropriate length and attached to fittings. Standard scaffolds were still electrospun to be $3 \mathrm{~mm}$. Changes in scaffold lengths were tracked throughout the preshrink and cultivation.

At the 3 and 7-day timepoints, BVMs were removed from the bioreactors and cut in half. One half of the scaffold was fixed in $2.5 \%$ glutaraldehyde and used for SEM imaging, while the other half was immunostained using the apoptosis staining protocol. Image quantification was performed for all three channels using the methods described in Section 3.2.3.

\subsection{Results}

Due to the large number of images, only representative images will be included in this section as needed. A larger set of images can be found in Appendix M, Appendix N, Appendix O, and Appendix P. 


\subsubsection{Stain Verification}

Before applying the apoptosis stain kit to any experiment, the three stains included in the kit were verified and optimized on the confocal microscope. Table 5 shows images of each case necessary to verify that no bleed through occurred between channels during imaging. Each row shows the same imaging area taken with a different laser in each channel. The three channels, Hoechst, Caspase, and Propidium Iodide, correspond to the three stains in the apoptosis kit. The "no stain" group in the first row serves to verify that no autofluorescence was present in the sample. The lack of signal in each channel shows that there was no autofluorescence from the HUVECs used in these images.

Table 5: Apoptosis Staining Kit Verification using HUVECs.

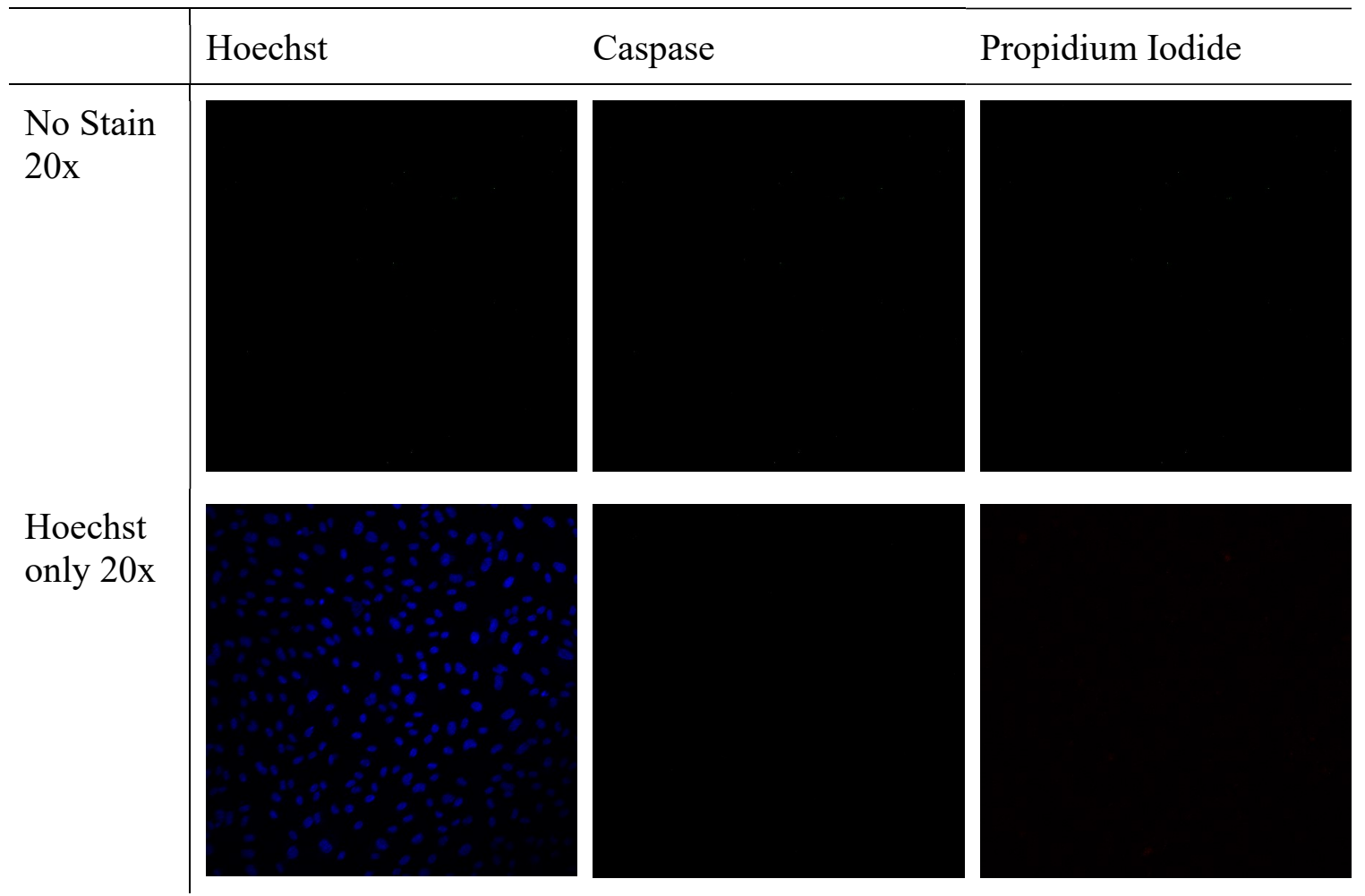




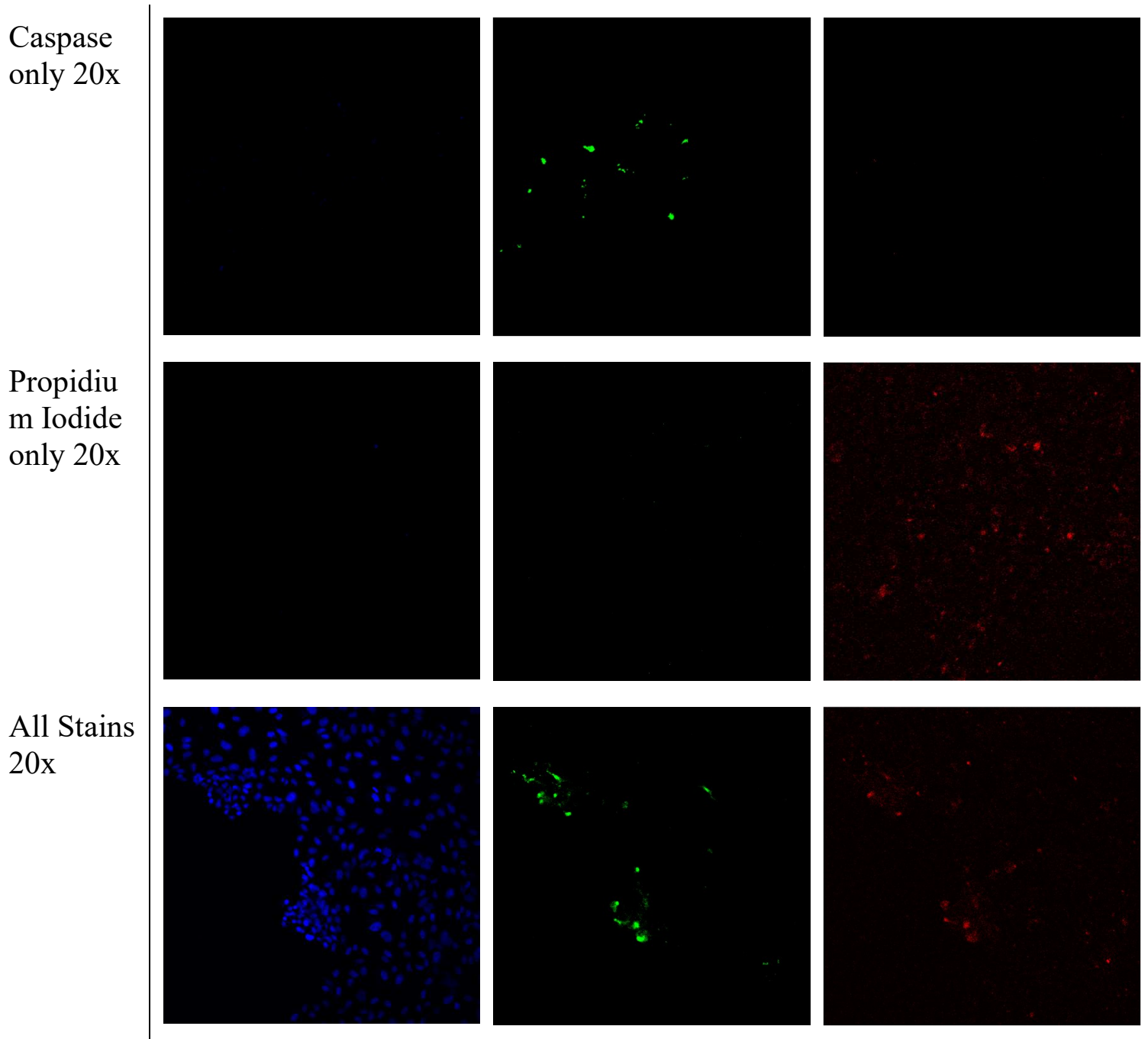

The middle three rows in Table 5 show samples that have only been stained with a single staining component from the kit. If bleed-through were occurring from one of the three stains, more than one channel would have signal in it. In this case, each row has a single image with signal in the correct channel. Therefore, no bleed-through was occurring between channels in this kit. The fifth row shows HUVECs that were stained with all three kit components, so each channel has signal in it. However, the Caspase channel has little signal in it, which is expected for untreated HUVECs in culture that should not be undergoing apoptosis. A positive control, in this case a treatment to induce 
apoptosis, was necessary in order to verify that the Caspase stain appropriately identifies apoptotic cells.

The two inducers of apoptosis, hydrogen peroxide and DMSO, were applied to HUVECs at increasing concentrations and lengths of time to determine a treatment that resulted in appropriate apoptosis detection. This treatment group should result in nearly all cells being labeled for apoptosis without completely killing the cells. This treatment should also result in cells stained in the cytosol and the nucleus, as the poly caspase stain used here should label caspases present in both areas of the cell. A sufficient apoptosis inducer should result in the labeling of the whole cell. Figure 41 and Figure 42 show treatment groups that were insufficient at inducing apoptosis. The 15\% DMSO treatment for 30 minutes in Figure 41 resulted in some signal in the cytosol of the cell. This signal, however, was not as strong as would be expected from a positive control, and no cells were stained in the nuclei. An important clarification is necessary to establish here: while these cells do not have sufficient caspase activity to be positive controls, these cells are still apoptotic. Any cell with caspase signal throughout the cytosol will still be considered apoptotic and quantified as so in the remaining experiments. All DMSO treatment groups were insufficient as the positive control, which can be seen in Appendix M. 


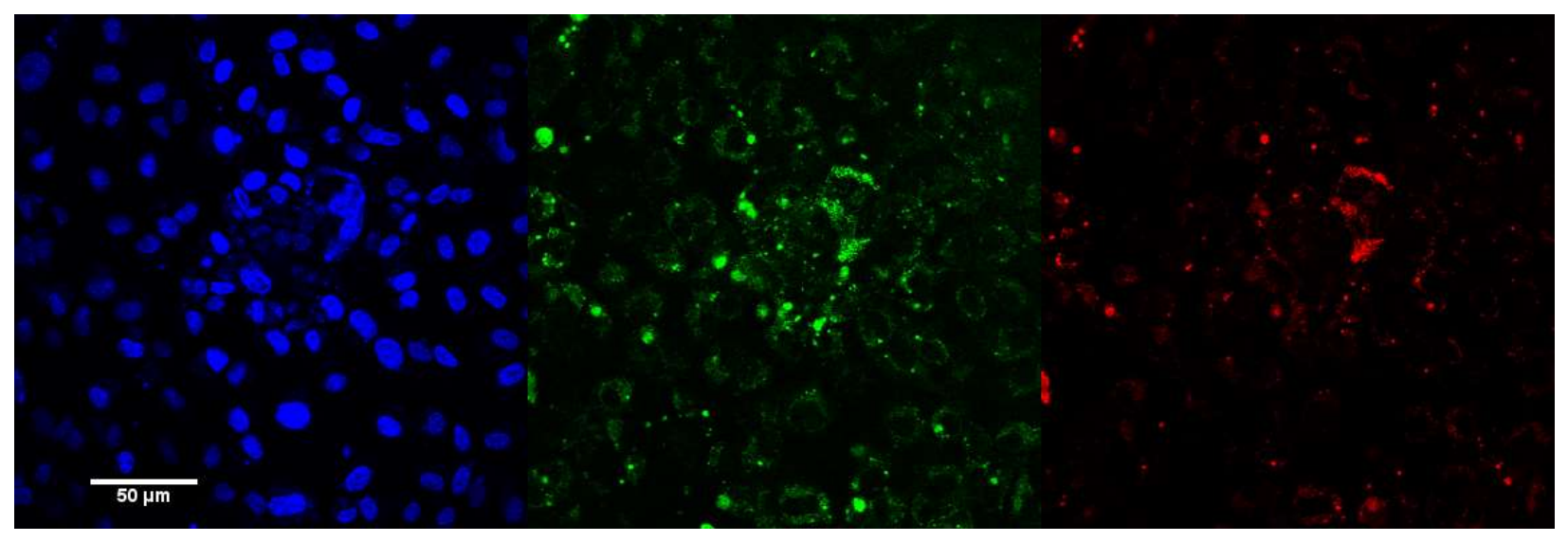

Figure 41: Ineffective Positive Control DMSO 15\% 30 Minutes. HUVECs treated with 15\% DMSO for 30 minutes in Hoechst (left), Caspase (middle) and Propidium Iodide (right) channels. These images indicate that 15\% DMSO treatment for 30 minutes was not a strong inducer of apoptosis. High resolution images can be obtained from Dr. Cardinal.

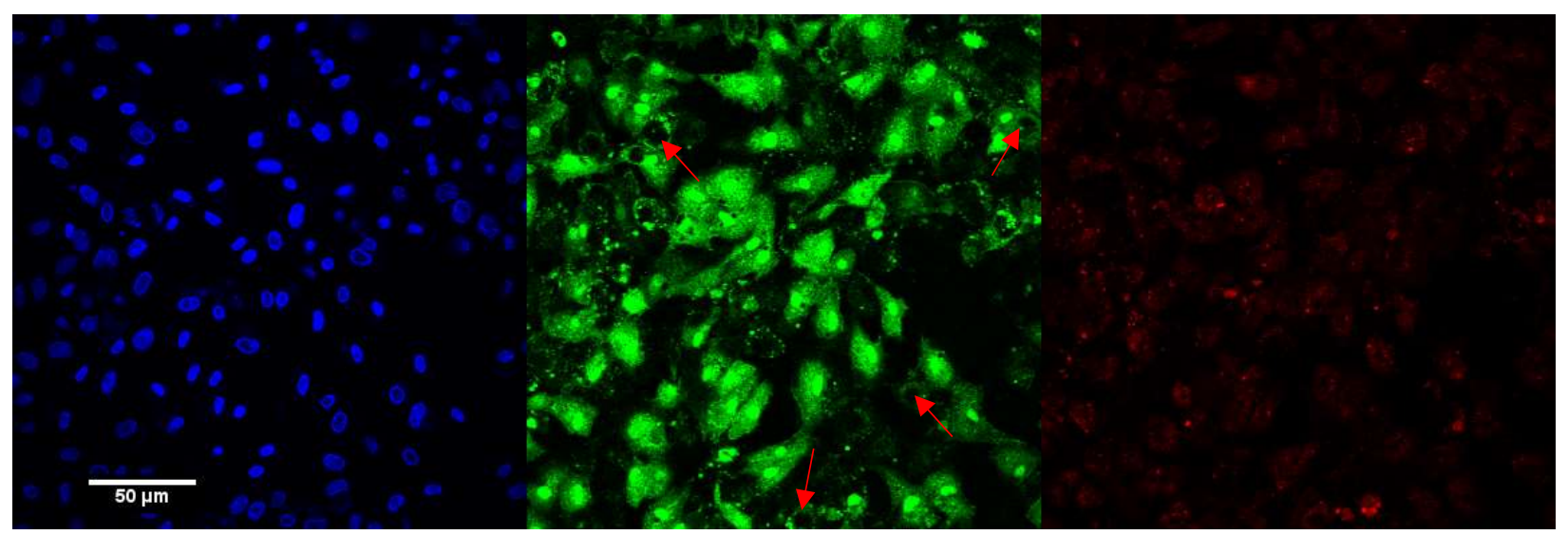

Figure 42: Ineffective Positive Control Hydrogen Peroxide 1\% 2 Minutes. HUVECs treated with $1 \%$ hydrogen peroxide for 2 minutes in Hoechst (left), Caspase (middle) and

Propidium Iodide (right) channels. These images indicate that $1 \%$ hydrogen peroxide treatment for 2 minutes was not a strong inducer of apoptosis. Red arrows point to cells that are stained for Caspase in the cytosol but not the nuclei. High resolution images can be obtained from Dr. Cardinal.

The 1\% hydrogen peroxide treatment for 2 minutes shown in Figure 42 was insufficient as well; while many of the cells were stained in the cytosol and nucleus, there were some cells that lacked the stain in the nucleus. Many of the other hydrogen peroxide treated groups were also insufficient, which can be seen in Appendix M. However, two 
hydrogen peroxide groups were identified as sufficient positive controls, which are displayed in Figure 43 and Figure 44. Both groups had the cytosol and nucleus of all cells intensely labeled for caspase activity without resulting in large amounts of dead cells. These two treatment groups were used as positive controls in the cytotoxicity elution tests.

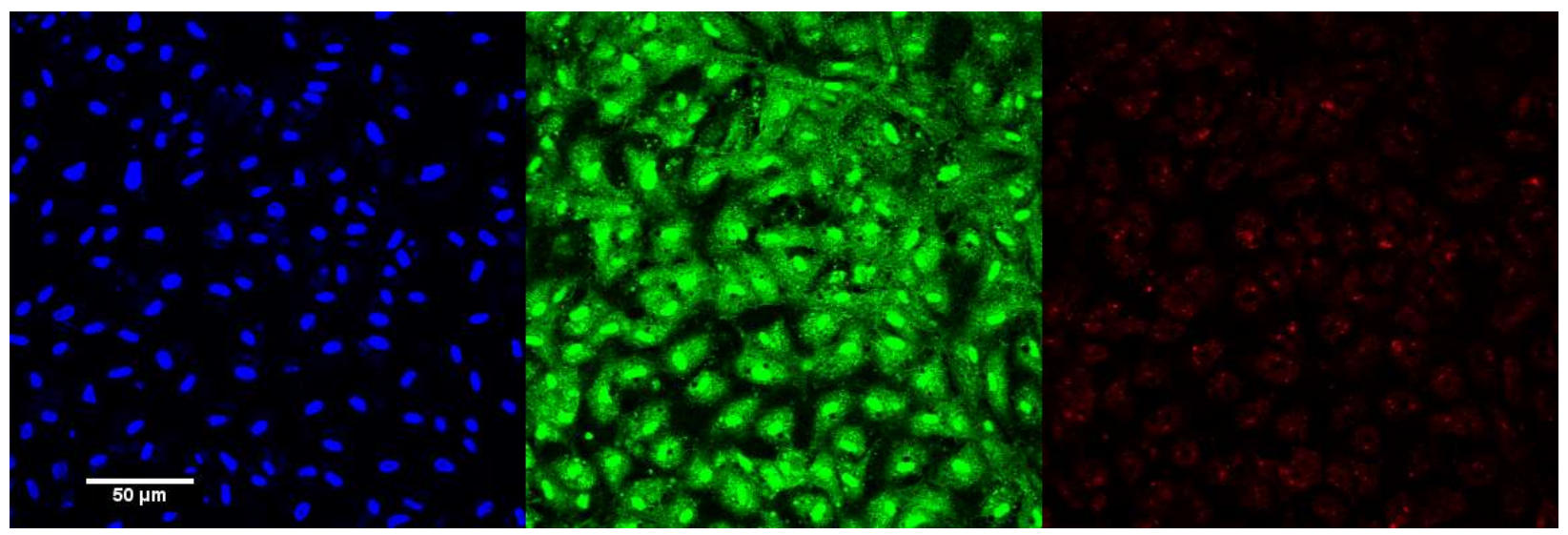

Figure 43: Effective Positive Control Hydrogen Peroxide 0.5\% 5 Minutes. HUVECs treated with $0.5 \%$ hydrogen peroxide for 5 minutes in Hoechst (left), Caspase (middle) and Propidium Iodide (right) channels. High resolution images can be obtained from Dr. Cardinal.

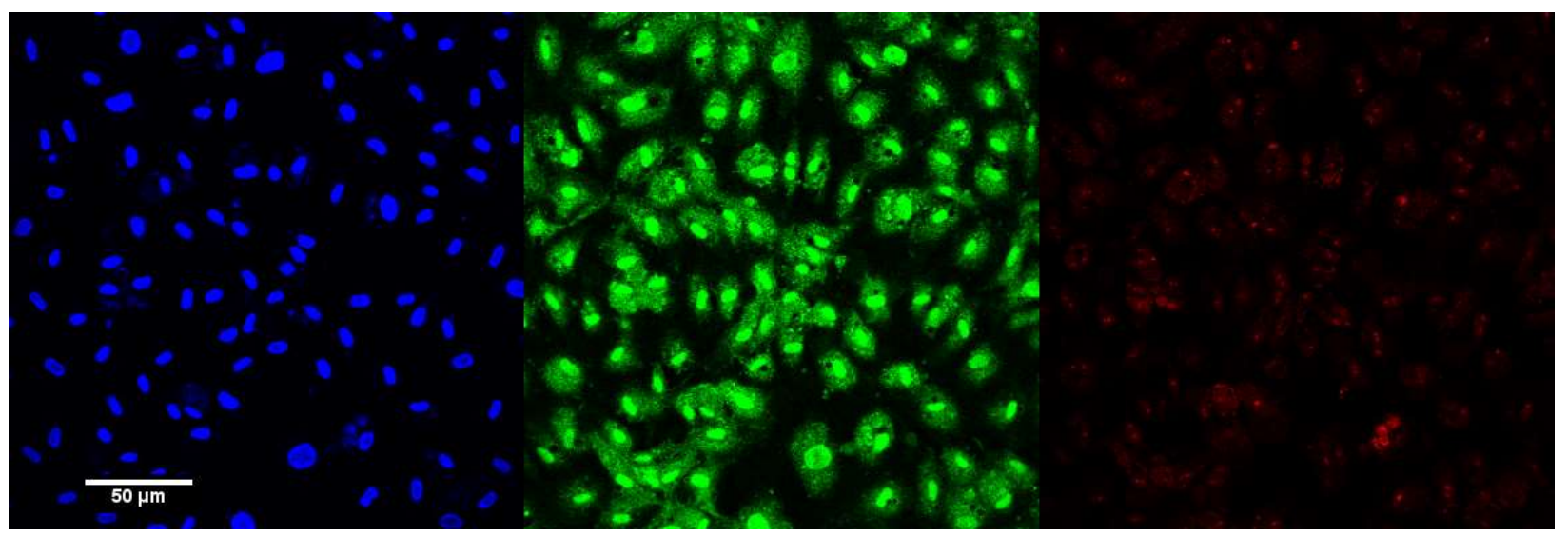

Figure 44: Effective Positive Control Hydrogen Peroxide 1\% 5 Minutes. HUVECs treated with $1 \%$ hydrogen peroxide for 5 minutes in Hoechst (left), Caspase (middle) and Propidium Iodide (right) channels. High resolution images can be obtained from Dr. Cardinal. 


\subsubsection{Cytotoxicity Elution Assay}

The primary purpose of this study was to determine if the eluted contents from the BTEAC salt scaffolds resulted in higher numbers of apoptotic cells than standard scaffolds. Two elution methods were initially investigated, both of which were recommended by ISO 10993, and evaluated based on scaffold degradation and $\mathrm{pH}$. Elutions were conducted at $70^{\circ} \mathrm{C}$ for 24 hours and $50^{\circ} \mathrm{C}$ for 72 hours. Figure 45 shows scaffolds after removal from elutions, Figure 46 shows the color of the eluted media, and Table 6 displays the $\mathrm{pH}$ of elution from different scaffold types from each protocol. Each of these figures will be described and included below.

The $70^{\circ} \mathrm{C}$ elution for 24 hours, shown in Figure 45A, resulted in warped and crumbling scaffolds, many of which began to flake into the eluted media upon removal. The $50^{\circ} \mathrm{C}$ elution for 72 hours resulted in stiffening and brittleness of the scaffolds, but they retained their shape and did not crumble during removal from the elution. There was also a stark difference in media color from the two elution protocols, in which the color was independent of the type of scaffold used. Figure 46B shows a yellow coloration to the eluted media, while the eluted media in Figure 46C is pink in color. The pink color in the $50^{\circ} \mathrm{C}$ eluted media is like the color of the HUVEC media before the elution protocol. 


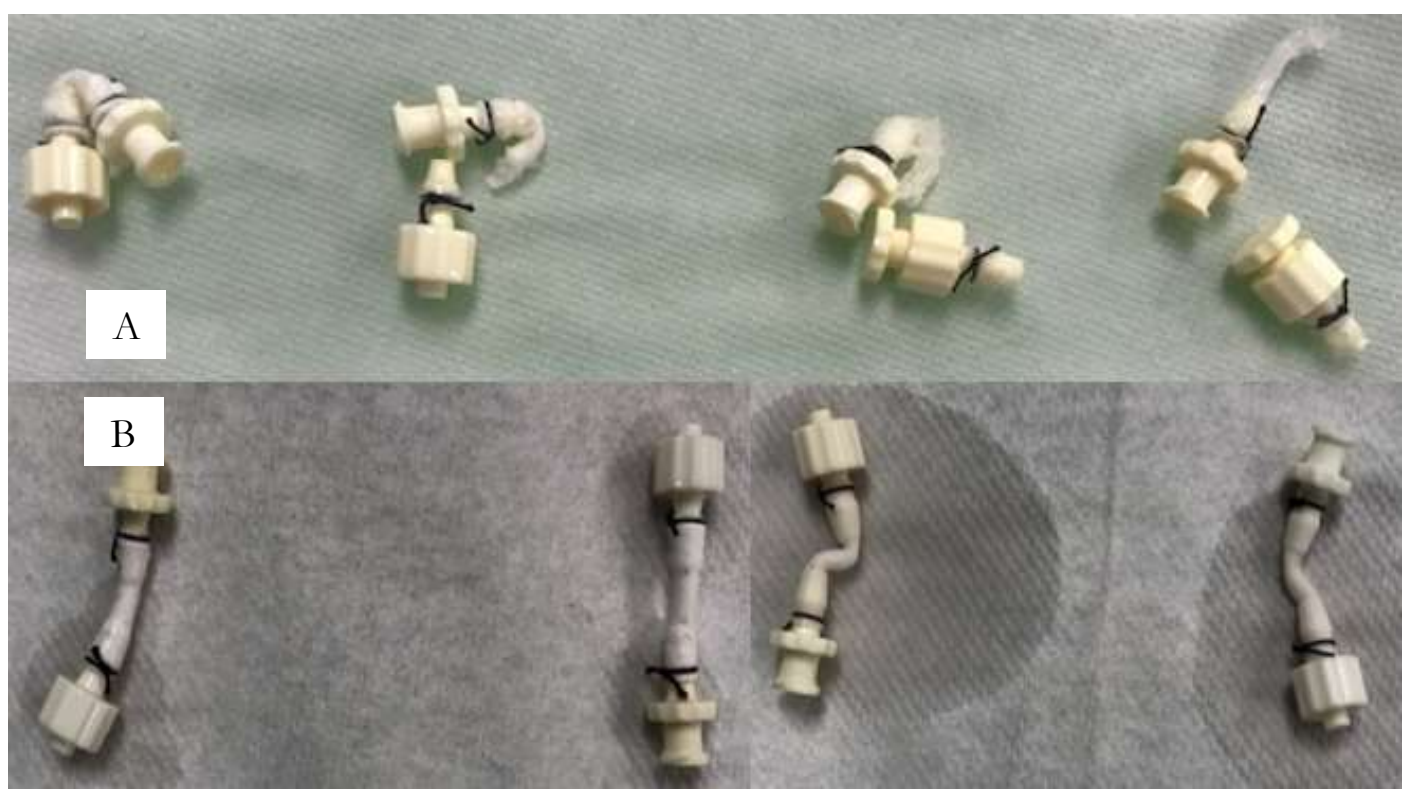

Figure 45: Scaffolds After Elution Treatment. (A) Scaffolds after $70^{\circ} \mathrm{C}$ elution for 24 hours. (B) Scaffolds after $50^{\circ} \mathrm{C}$ elution for 72 hours. In both A and B, two scaffolds on the left are $3 \%$ salt scaffolds and the scaffolds on the right are standard scaffolds.

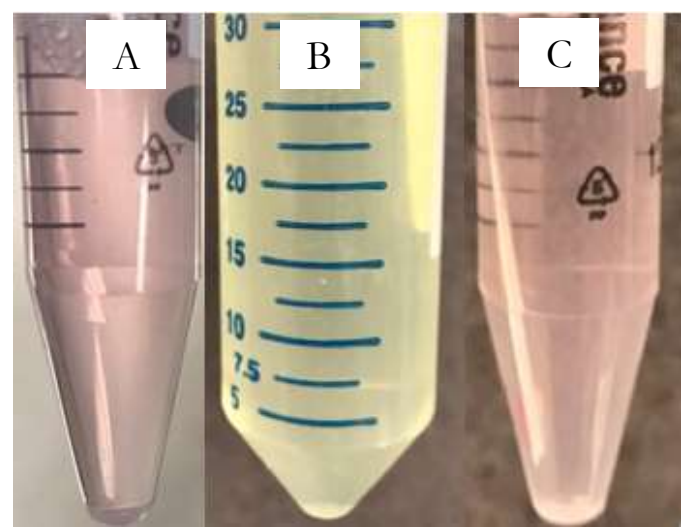

Figure 46: Media After Elution Treatment. (A) Standard media that has not been eluted. (B) Media eluted at $70^{\circ} \mathrm{C}$ for 24 hours. (C) Media eluted at $50^{\circ} \mathrm{C}$ for 72 hours.

A change in color from pink to yellow generally indicates a change in $\mathrm{pH}$ towards an acid. Based on the change in media color in the $70^{\circ} \mathrm{C}$ elution, it would be expected that this media would be acidic. This was confirmed after testing the $\mathrm{pH}$, seen in Table 6 . For the $70^{\circ} \mathrm{C}$ elution the standard scaffold resulted in a $\mathrm{pH}$ of 7.0 , while the $3 \%$ salt scaffold had a $\mathrm{pH}$ between 7.0 and 7.5 . The $50^{\circ} \mathrm{C}$ elution had consistent $\mathrm{pH}$ values of 7.5 for both 
scaffold types. These values were similar to the non-eluted media, which had a pH of 7.5. It was unsurprising that some of the elutions were slightly acidic, as PLGA breaks down into acidic byproducts ${ }^{168}$. Based on the structural retention of the scaffolds after the elution and no observations of a color or $\mathrm{pH}$ change in the eluted media, it was decided to use the $50^{\circ} \mathrm{C}$ elution for 72 hours during the cytotoxicity study.

\section{Table 6: pH of Elutions from Treatment Methods.}

\begin{tabular}{c|ccc}
\hline & Non-eluted Media & $\begin{array}{c}70^{\circ} \mathrm{C} \text { Elution for } 24 \\
\text { hours }\end{array}$ & $\begin{array}{c}50^{\circ} \mathrm{C} \text { Elution for } 72 \\
\text { hours }\end{array}$ \\
\hline $\begin{array}{c}\text { Standard Scaffold } \\
\text { Average }\end{array}$ & 7.5 & 7.0 & 7.5 \\
$\begin{array}{c}\text { 3\% Salt Scaffold } \\
\text { Average }\end{array}$ & & $7.0-7.5$ & 7.5
\end{tabular}

The eluted media from each type of scaffold was then used to treat HUVECs for 24 or 48 hours before the samples were stained and imaged. The quantification methods for each channel differed. An automated cell counter for the Hoechst channel was used to count the nuclei; however, it was unknown what magnification was appropriate for automated counting. Two magnifications were investigated, 20x and 40x, to determine the accuracy of the automated counter compared to a manual count. It was found that the automated cell count at a $20 \mathrm{x}$ magnification had an error of $40.02 \%$, while the automated cell count at 40x had an error of 9.15\%. This data can be found in Appendix Q. With such a high margin of error from the 20x magnification automated counting, images at 40x magnification were used for the cytotoxicity assay.

Unlike the Hoechst channel, the Caspase and Propidium Iodide channels had to be quantified manually. In order to aid the counter, it was decided to eliminate pixels below a certain intensity to emphasize cells that were truly apoptotic or dead. This would help 
reduce background noise and increase the accuracy of Caspase or Propidium Iodidepositive cell counts. In order to determine the range of pixel intensities to eliminate, a manual cell count was performed on Caspase and Propidium Iodide channels at increasing pixel reduction values. The cell count after each pixel reduction was then compared to the original number of Caspase or Propidium Iodide-positive cells. The cell count percent change for the Caspase channel and Propidium Iodide channel is presented in Figure 47. It was hoped that a plateau region would be obvious in both graphs, which would indicate an appropriate minimum pixel intensity. However, neither channel had a plateau, so it was decided to limit the loss of signal positive cells to less than $10 \%$. Therefore, a pixel intensity reduction of 15 and 20 were used for the Caspase and Propidium Iodide channels, respectively. The raw data for these graphs can be found in Appendix R.
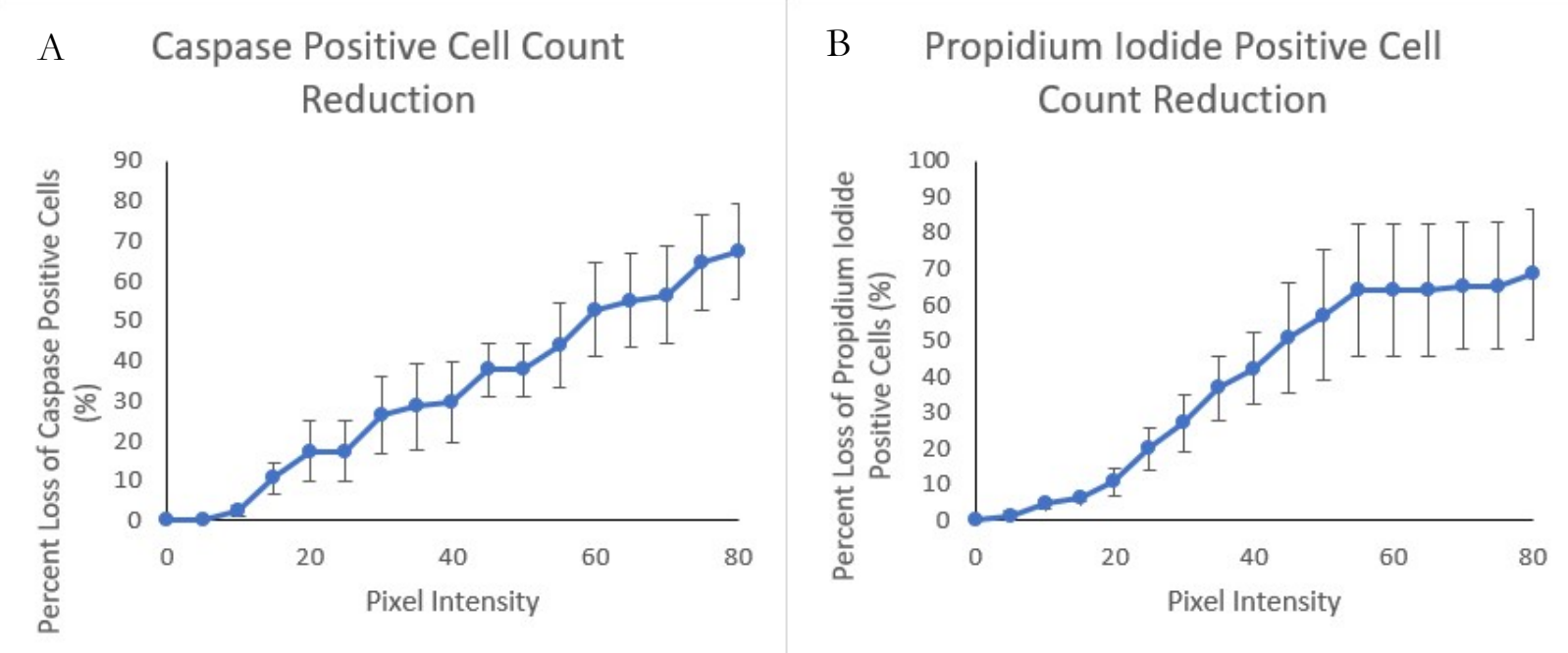

Figure 47: Cell Count Reduction Curves. (A) Caspase-positive cells percent change as minimum pixel intensity increases. (B) Propidium Iodide-positive cells percent change as minimum pixel intensity increases. 
With the image quantification methods established, the raw images of HUVECs treated with either a dilution or non-dilution of eluted media from standard or salt scaffolds could be quantified and analyzed. A negative control of HUVECs treated with normal EC media was included, as well as two positive control groups using $0.5 \%$ and $1 \%$ hydrogen peroxide for 5 minutes. Representative raw images from this study can be found in Appendix N.

Figure 48 displays the percent of Caspase-positive cells in each treatment group. A three-way ANOVA was run for treatment length, dilution, and scaffold type. At each treatment length there was no difference between the $50 \%$ dilution and no-dilution groups regardless of scaffold type. For each treatment length, the negative control, $50 \%$ dilution standard scaffold, and no-dilution standard scaffold groups had lower percentages of Caspase-positive cells than the 50\% dilution salt scaffold and no-dilution salt scaffold groups. Furthermore, for each treatment length, the 50\% dilution salt scaffold and nodilution salt scaffold groups had lower percentages of Caspase-positive cells than either positive control groups. In summary, the negative control and standard scaffold groups had less caspase activity than the salt scaffold groups, which had less activity than the positive control groups. 


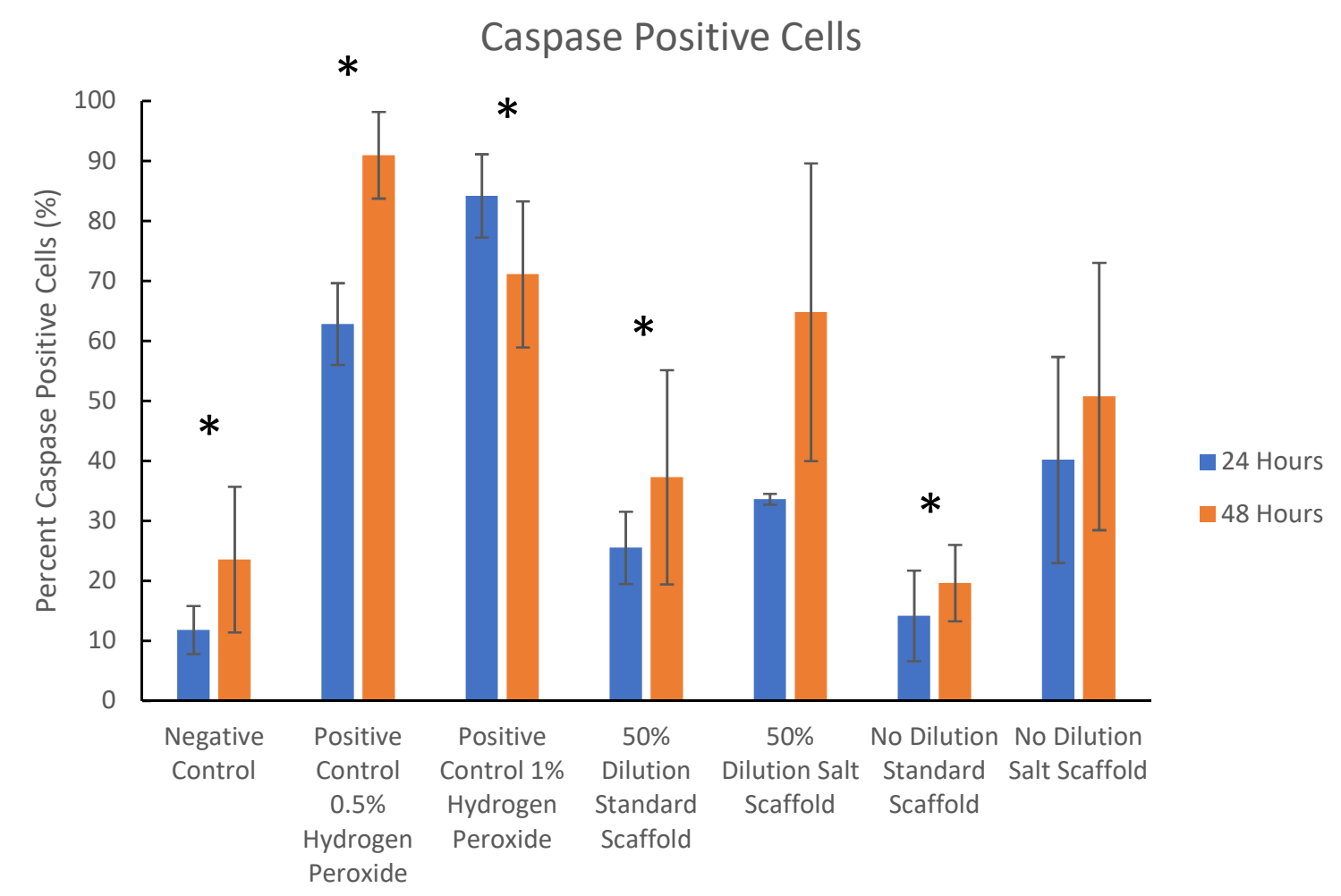

Figure 48: Cytotoxicity Elution Assay Percent Caspase Positive Cells. Percentage of Caspase positive cells in each treatment group. The * indicates a difference in the 24hour timepoint compared to the 24 hour no dilution salt scaffold group and a difference in the 48-hour timepoint compared to the 48 -hour no dilution salt scaffold group $(\mathrm{p}<0.001)$.

In order to eliminate rates of naturally occurring apoptosis from the data, the normal scaffold and salt scaffold treatment groups were normalized to the negative control. This is shown in Figure 49, and this normalization eliminated significant differences seen previously in Figure 48. No differences in percent caspase-positive cells were found between time points, within timepoints of the same treatment, between scaffold types, nor between dilution groups. 


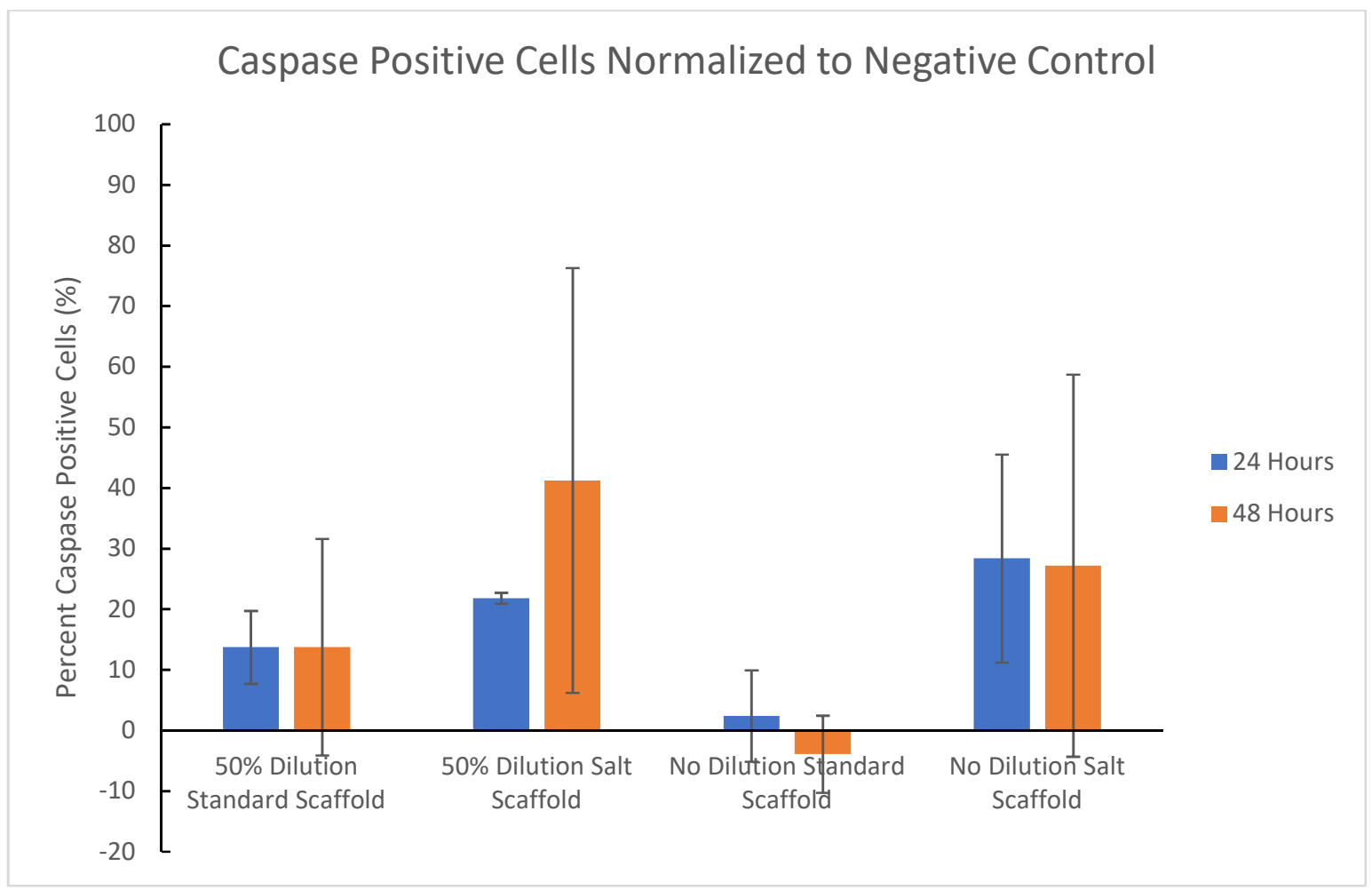

Figure 49: Normalized Cytotoxicity Elution Assay Percent Caspase Positive Cells.

Percentage of Caspase positive cells normalized to the negative control group. No differences were observed between treatment groups nor timepoints.

Figure 50 shows the percent of Propidium Iodide positive cells per treatment group, which includes a negative control and two positive controls. All treatment groups had a higher percent of Propidium Iodide positive cells at the 48-hour treatment length than at 24 hours. No differences were seen between treatment groups at 24 hours. For the 48-hour treatment length, there was no difference between the positive control groups and the $50 \%$ dilution salt scaffold. However, the no dilution salt scaffold group had a lower percentage of Propidium Iodide positive cells than the $50 \%$ dilution salt scaffold group. Similarly, in the 48-hour treatment length, the no dilution standard scaffold had a lower percentage of Propidium Iodide positive cells than both the $50 \%$ dilution standard scaffold and the negative control group. These relationships between dilution and 
undiluted groups is surprising, as it is opposite of how a dilution group is expected to affect cell death if the elution does have an effect.

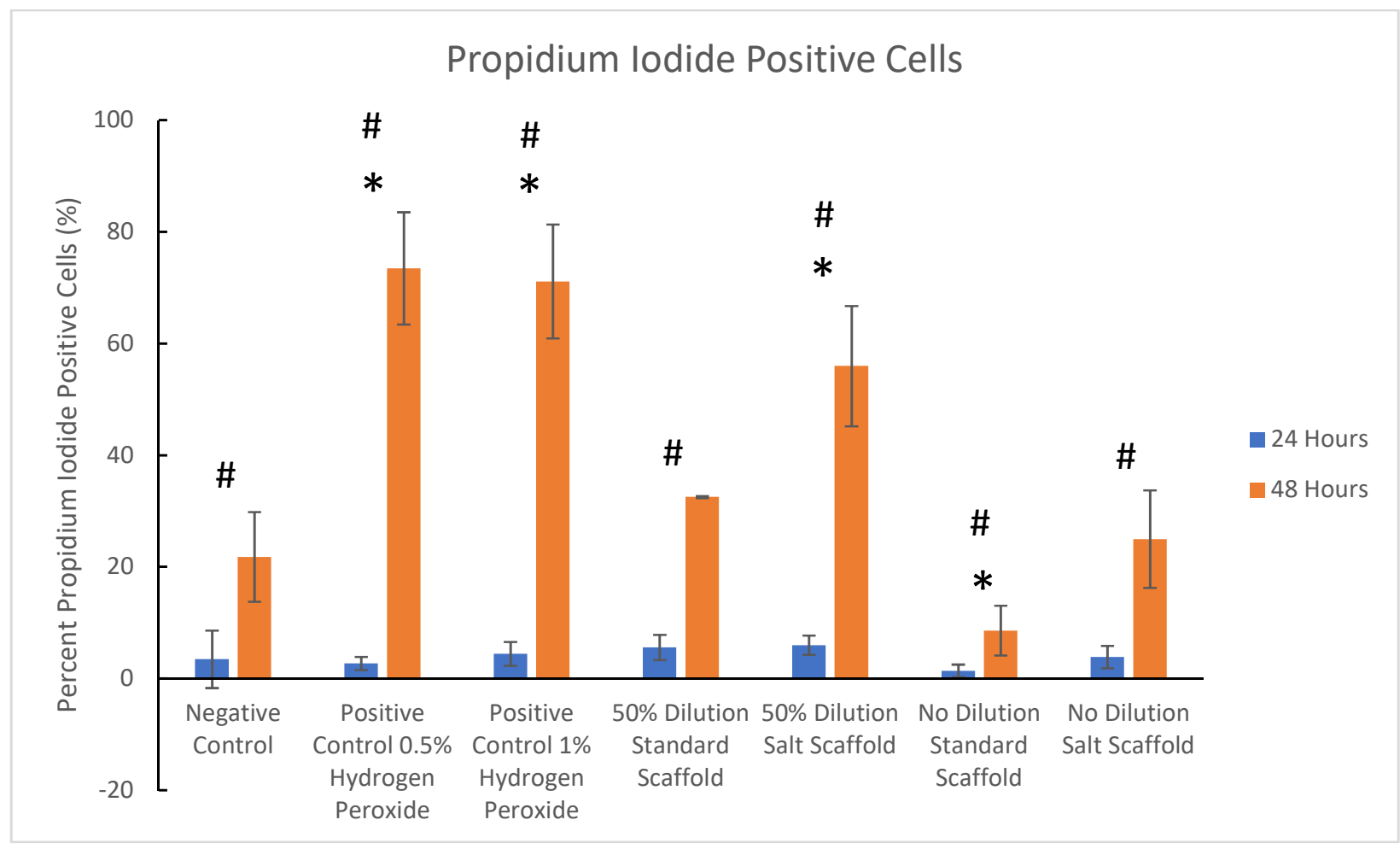

Figure 50: Cytotoxicity Elution Assay Percent Propidium Iodide Positive Cells. Percentage of Propidium Iodide positive cells in each treatment group. The * indicates a difference in percentage at 48 hours compared to the no dilution salt scaffold group at 48 hours $(\mathrm{p}<0.05)$. The \# indicates a difference between the 24 and 48 -hour time point within the group $(\mathrm{p}<0.05)$.

Like with the Caspase group, the treatment groups for Propidium Iodide positive cells were normalized to the negative control group to eliminate natural cell death rates. Figure 51 displays these results after normalization. This normalization did not eliminate the pattern of dilution effects seen previously in Figure 50. At 48 hours, both the standard scaffold and salt scaffold dilution groups had more Propidium Iodide positive cells than their corresponding undiluted groups. At 24 hours, no difference persisted between all treatment groups. 


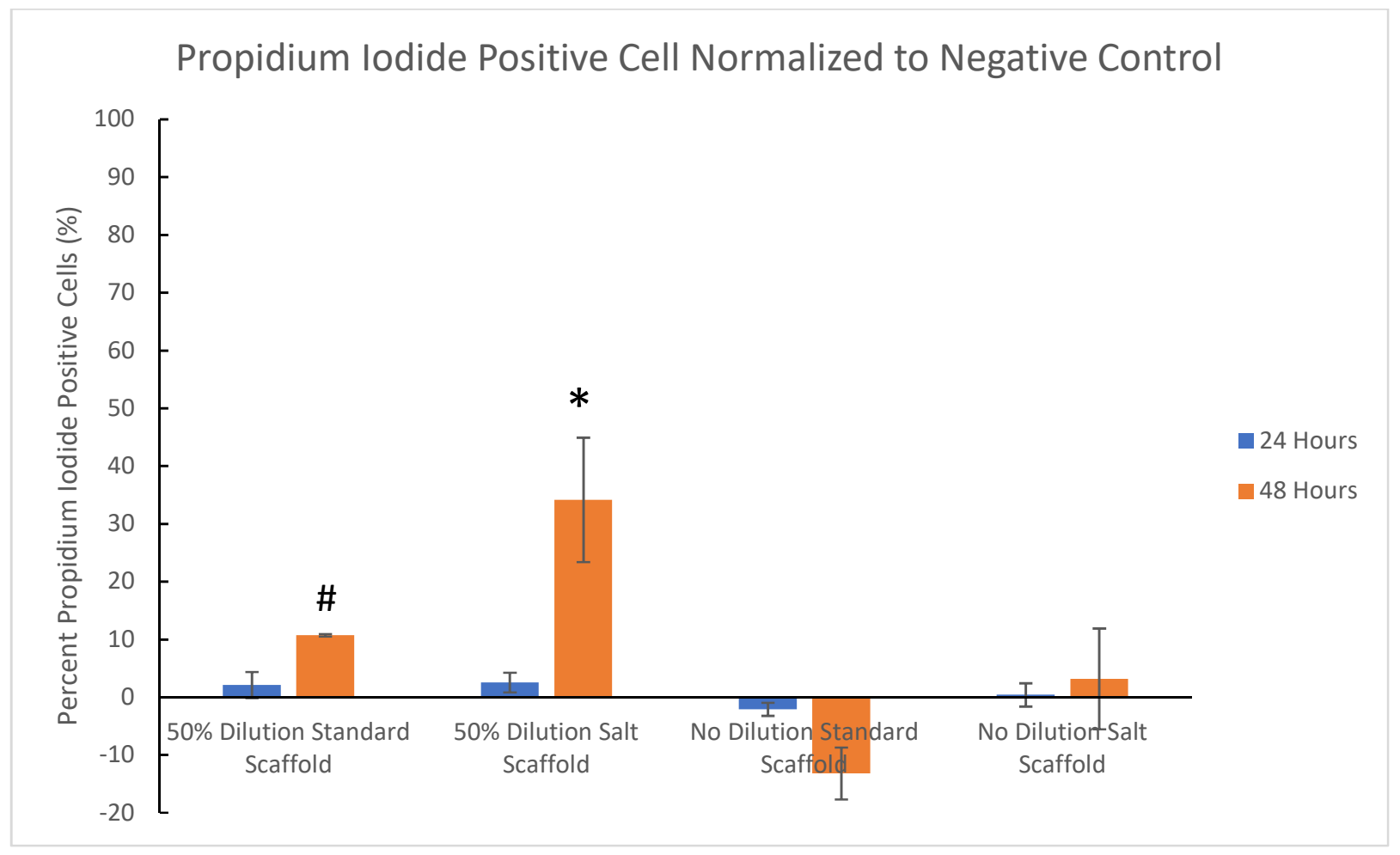

Figure 51: Normalized Cytotoxicity Elution Assay Percent Caspase Positive Cells. Percentage of Propidium Iodide positive cells normalized to the negative control group. The * indicates a difference compared to all other groups at both timepoints $(p<0.01)$. The \# indicates a difference compared to the 48-hour no dilution standard scaffold group $(\mathrm{p}<0.01)$.

\subsubsection{Cell Adhesion and Viability Assay}

The cytotoxicity elution assay assessed cell apoptosis and death without direct scaffold contact. While these toxicity measurements are useful, other metrics are necessary to assess novel scaffolds, especially regarding initial cell interactions. Therefore, the cell adhesion assay assessed cell coverage and scaffold toxicity 4 hours after HUVECs had been sodded onto the scaffolds. Some data regarding cell deposition could be collected without imaging through cell counts. Estimations of cell sodding numbers are shown in Table 7 . While this data does not visually measure cell adhesion, it is an indirect calculation of how many cells remained sodded to the scaffold. The 
standard scaffolds were sodded with 9.02 million cells, while the salt scaffolds were only sodded with 3.76 million cells. The difference in the number of cells sodded is because, unlike the standard scaffolds, only a portion of the desired cell solution could be injected into the salt scaffolds due to pressure buildup. This pressure could be a result of cells clogging the smaller pores of the salt scaffolds. Interestingly, a much higher percent of the sodded cells was collected from the petri dishes of salt scaffolds even though less cells were sodded. This would suggest that less of the cells sodded to the salt scaffolds remained in the scaffolds, or that less cells are needed to sod salt scaffolds to create a confluent cell lining.

Table 7: Short-term Adhesion Assay Cell Sodding and Collection Data.

\begin{tabular}{|l|l|l|l|}
\hline & $\begin{array}{l}\text { Number of cells sodded } \\
\text { per scaffold } \\
\text { (cells/scaffold) }\end{array}$ & $\begin{array}{l}\text { Collected cells from } \\
\text { petri dish (cells) }\end{array}$ & $\begin{array}{l}\text { Percent of cells } \\
\text { collected (\%) }\end{array}$ \\
\hline $\begin{array}{l}\text { Standard } \\
\text { Scaffold }\end{array}$ & $9.02 \mathrm{E} 6$ & $1.17 \mathrm{E} 6$ & 12.97 \\
\hline $\begin{array}{l}\text { 3\% Salt } \\
\text { Scaffold }\end{array}$ & $3.76 \mathrm{E} 6$ & $1.59 \mathrm{E} 6$ & 42.29 \\
\hline
\end{tabular}

The immunofluorescent imaging of the scaffolds was a more direct and visual assessment of cell coverage. To estimate cell coverage, an assumption had to be made regarding the size of a cell nucleus compared to the size of the entire cell. The ratio of these measurements could then be used as a factor with which to multiply the area of cell nuclei by to estimate the area of cell coverage. Since images were being taken at $20 \mathrm{x}$ magnification, sample images from literature of HUVECs taken at 20x, seen in Figure 52, were measured in ImageJ to determine the average nuclei and whole cell size. It is unknown how these images may differ from the images taken from the 20x setting on the 
confocal microscope used in this aim, but this value was only intended to be an estimation, so some difference was expected. Raw area measurements can be found in Appendix S. The average nucleus was found to be $23.42 \%$ of the entire cell. Dividing $100 \%$ of the cell area by $23.42 \%$ results in the factor 4.27 , which was used as the multiplication factor to estimate cell coverage.

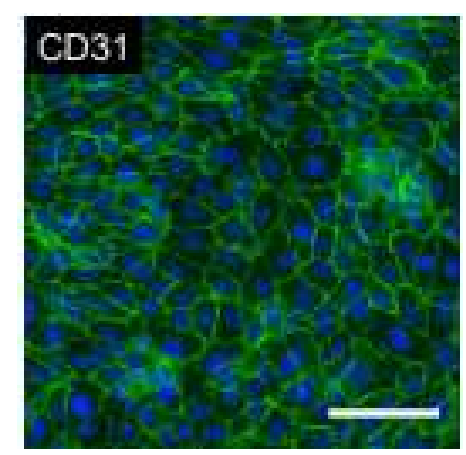

Figure 52: Sample 20x HUVECs. Images from literature of HUVECs taken at a 20x magnification. These cells were stained with junctional markers, which outline the entire cell ${ }^{169}$.

Figure 53 displays representative images of the Hoechst channel for standard and salt scaffolds. Upon visual comparison, the salt scaffolds had a far greater number of cells than the standard scaffolds. It also appeared that the standard scaffolds had decreasing cell coverage from proximal to distal ends, while the salt scaffold had similar coverage along the entire scaffold. Quantification data supported this visual assessment. 


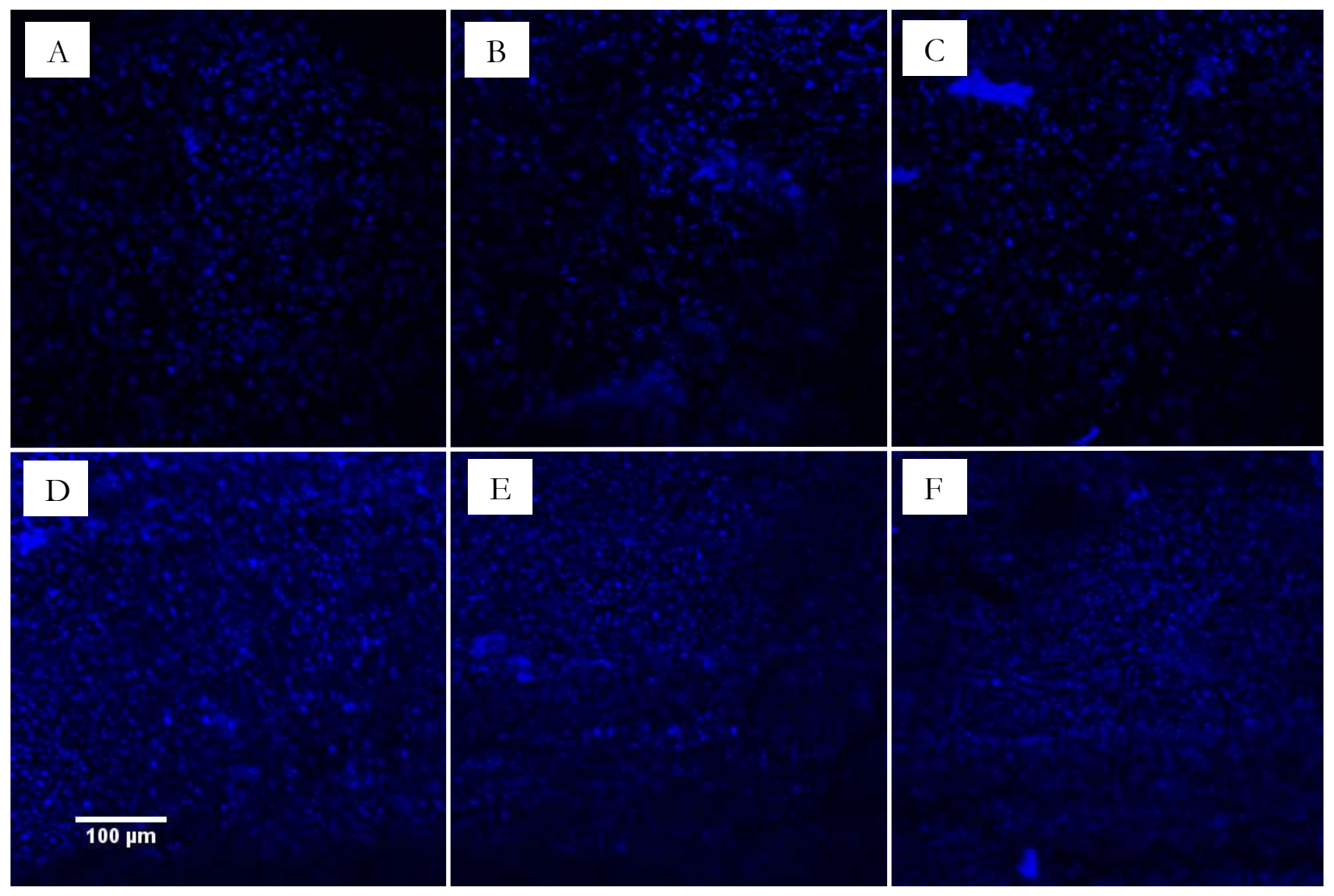

Figure 53: Standard and Salt Scaffolds Hoechst Channel. Standard scaffolds proximal (A), middle (B), and distal (C) show less coverage than salt scaffolds at proximal (D), middle (E), and distal (F) locations. High resolution images can be obtained from Dr. Cardinal.

Figure 54 compares estimated cell coverage between scaffold types at different areas of the scaffold. Salt scaffolds had higher coverage than standard scaffolds. There was also a trend of decreasing coverage along the length of the standard scaffold group. 


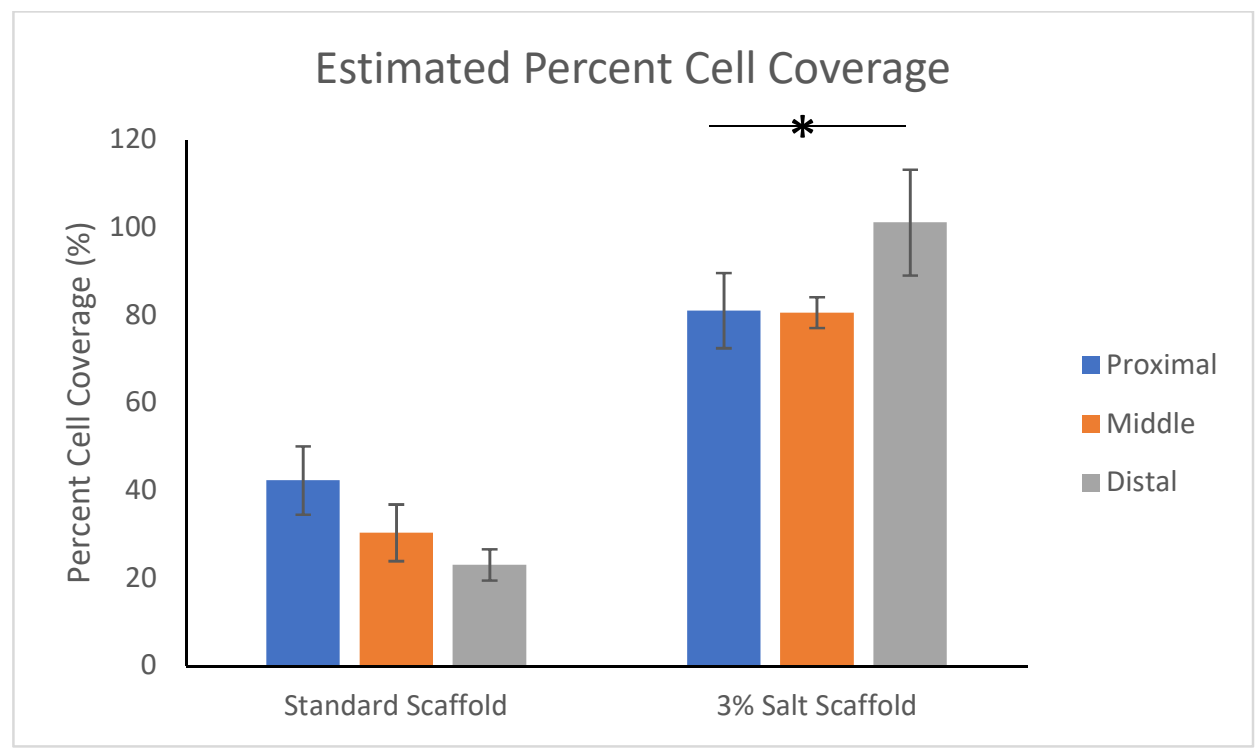

Figure 54: Short-term Adhesion Assay Percent Cell Coverage. Estimated percent cell coverage for standard and salt scaffolds on the proximal, middle, and distal areas of the scaffold. The * indicates a difference in coverage between the scaffold types $(p<0.001)$.

Apoptosis and cell death were also quantified using the Caspase and Propidium Iodide channels, respectively. Figure 55 shows representative images of Caspase and Propidium Iodide channels for the different scaffolds. Figure 56 shows that no differences were seen in the estimated percent of apoptotic cells between the standard scaffolds and salt scaffolds. Even though apoptotic cells showed no differences, there were differences in the percent of dead cells. Figure 57 shows that there was a higher estimated percent of dead cells in the salt scaffold group than the standard scaffold group. 


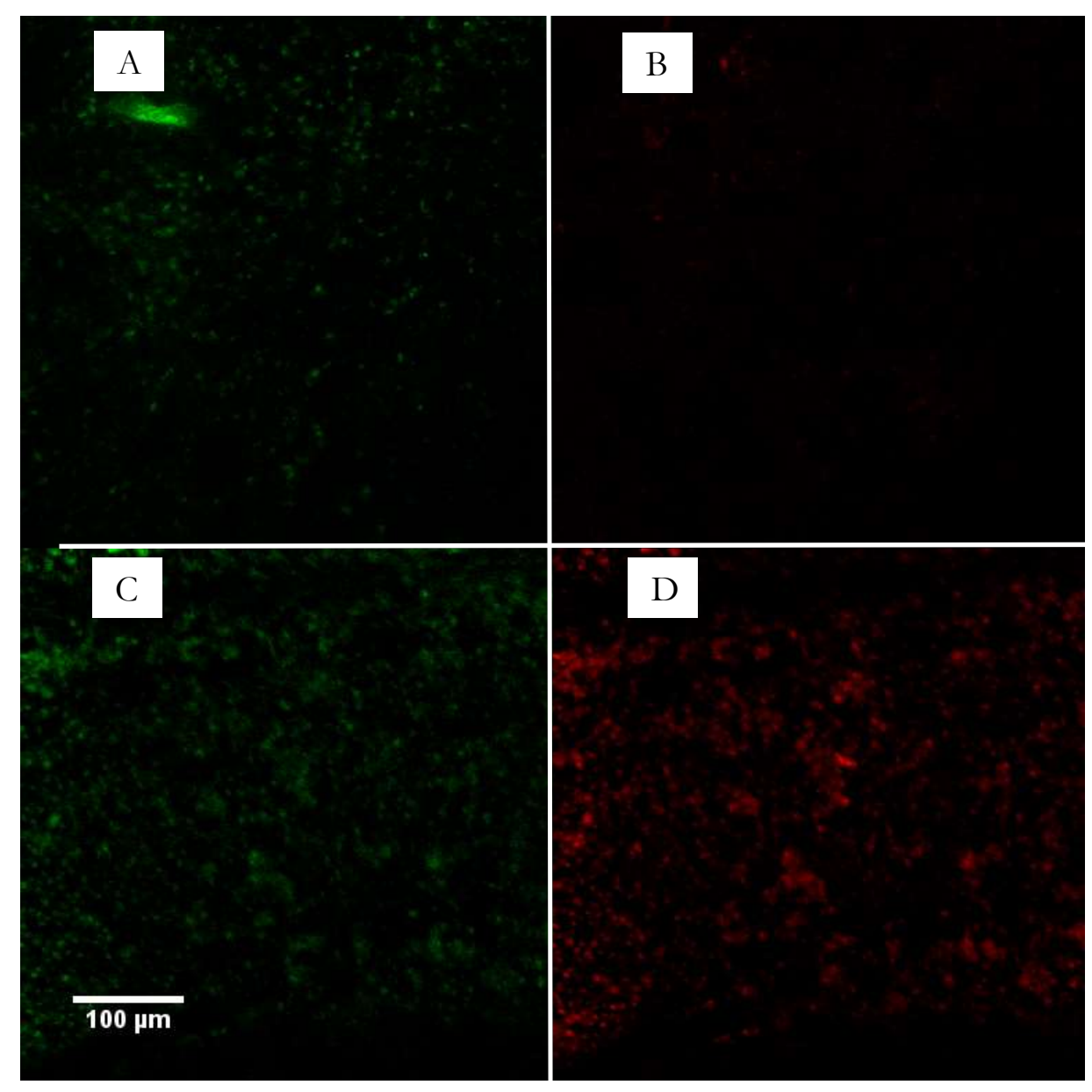

Figure 55: Caspase and Propidium Iodide Channels Short-term Adhesion Assay.

Salt and standard scaffolds at 20x magnification. (A) Caspase channel for standard scaffold. (B) Propidium Iodide channel for standard scaffold. (C) Caspase channel for salt scaffold. (D) Propidium Iodide channel for salt scaffold. High resolution images can be obtained from Dr. Cardinal. 


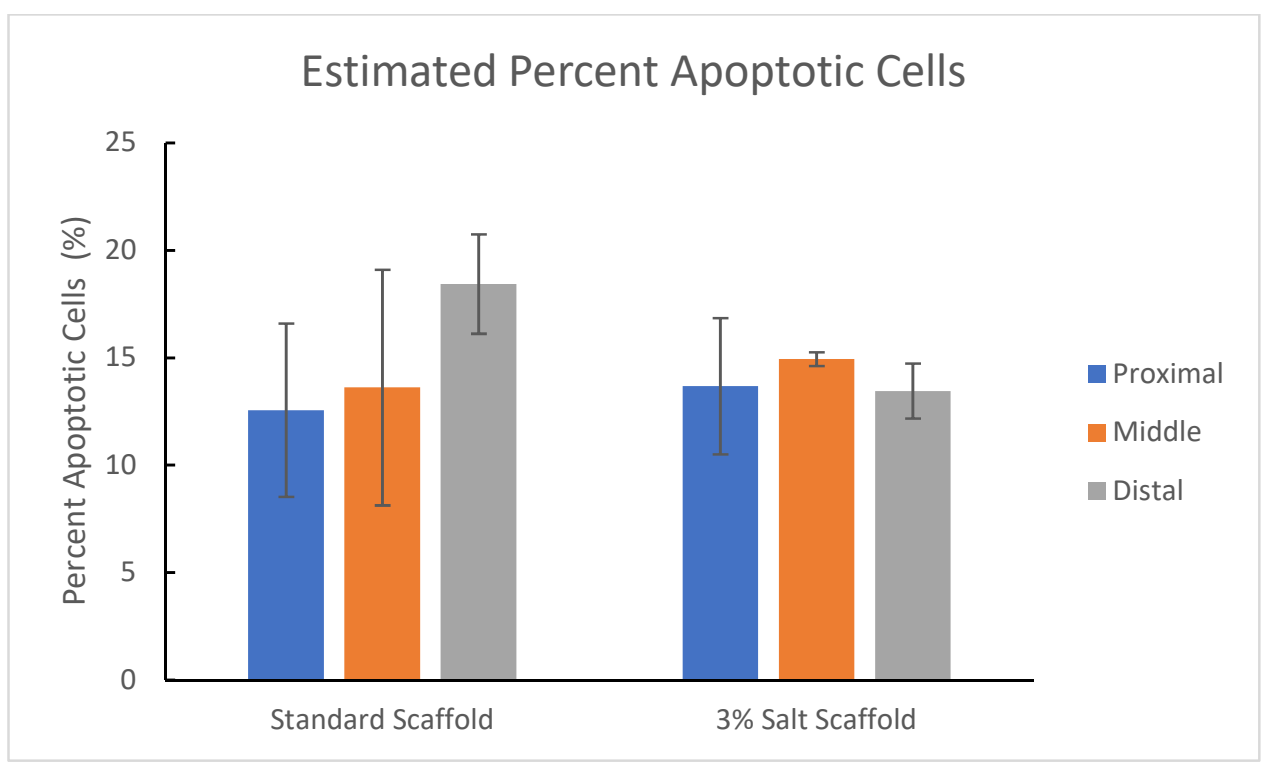

Figure 56: Short-term Adhesion Assay Percent Apoptotic Cells. Estimated percent apoptotic cells for standard and salt scaffolds on the proximal, middle, and distal areas of the scaffold. No differences were observed between scaffold types or areas of the scaffold.

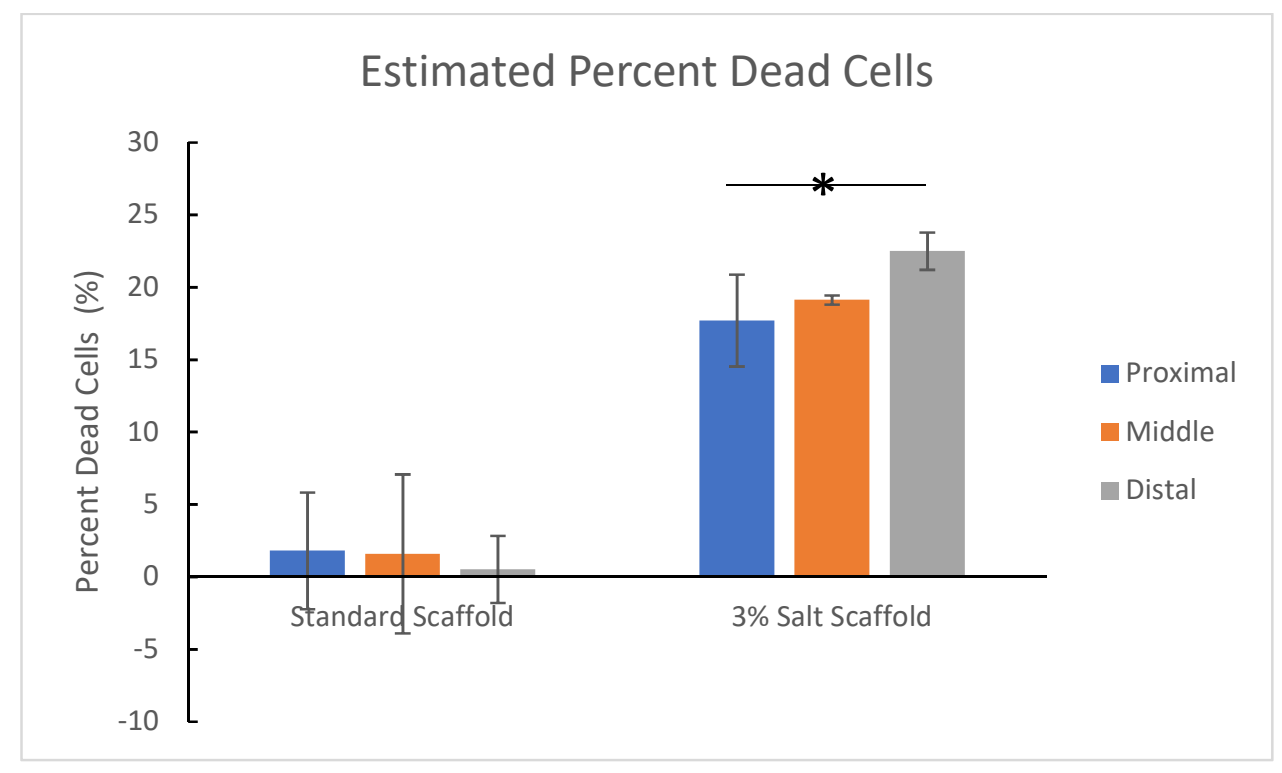

Figure 57: Short-term Adhesion Assay Percent Dead Cells. Estimated percent dead cells for standard and salt scaffolds on the proximal, middle, and distal areas of the scaffold. The * indicates a difference in dead cells between the scaffold types $(p<0.001)$. 


\subsubsection{BVM Cell Coverage and Viability Assay}

The 4-hour time point was useful for assessing immediate cell attachment and interactions with the scaffold. However, to implement novel scaffolds these scaffolds need to prove useful in BVMs for multiple days. This experiment investigated how low and high percentage salt scaffolds compare to standard scaffolds at 3 and 7-day timepoints. Since this assay has not been previously conducted on standard scaffolds, an ePTFE scaffold was added as a negative control with which to compare all electrospun scaffold types. At each time point, 2 vessels of each electrospun scaffold type were fabricated. Unfortunately, one $0.5 \%$ vessel at the 7 -day time point was lost due to contamination.

Since it was previously noted that the salt scaffolds shrink during sterilization, changes in scaffold size were measured throughout the fabrication of the BVMs. Measurements of salt scaffold lengths were taken before and after preshrinking, and all scaffolds were measured before and after BVM cultivation. Figure 58 shows the changes in salt scaffolds from the preshrink and cultivation. The scaffold groups display a proximal and distal, which is differentiating between the end of the mandrel in which the scaffold was sourced since each mandrel produces two scaffolds. Scaffold length changed more during preshrinking than during cultivation, which suggests that the preshrinking is useful as a method of reducing salt scaffold shrinking during cultivation. The change in length for all scaffolds after BVM cultivation is provided in Figure 59, which shows that both salt scaffold groups shrank more than standard scaffolds. Surprisingly, the standard scaffolds slightly expanded in length during the BVM cultivation. 


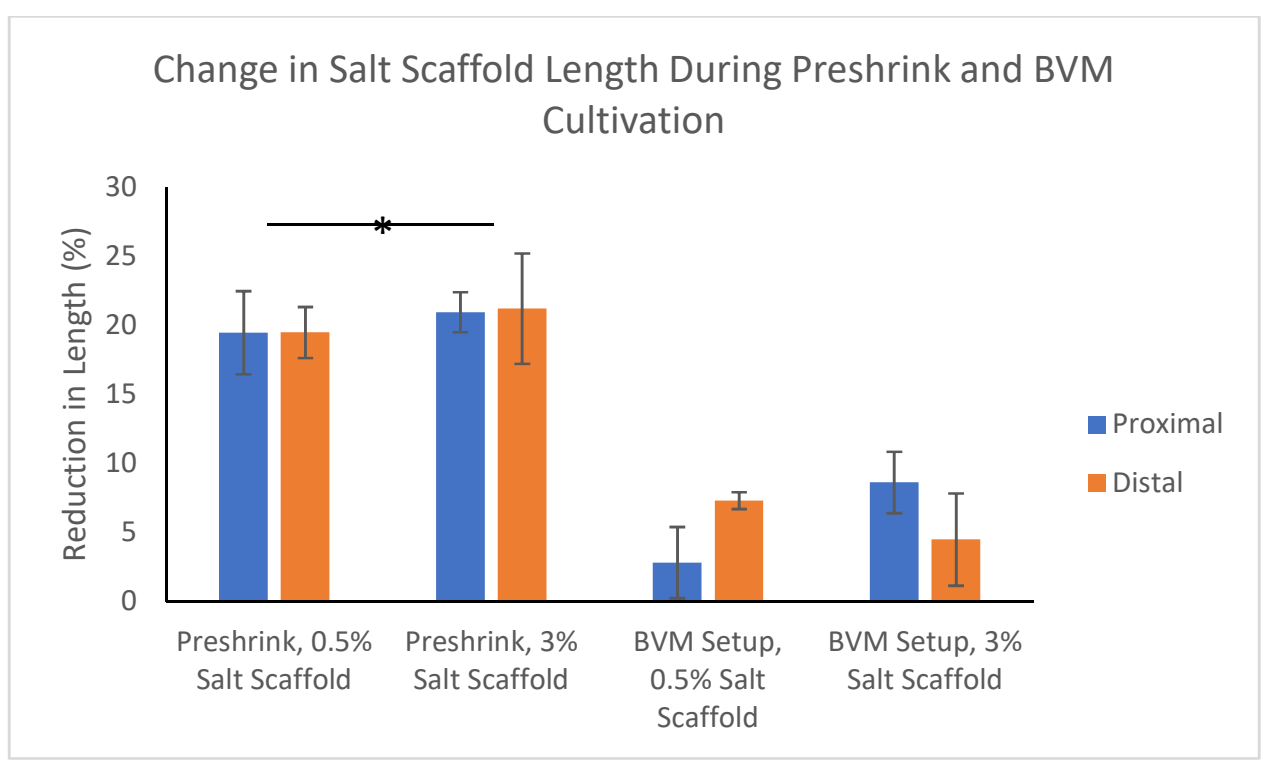

Figure 58: Salt Scaffold Shortening Before and After BVM Cultivation. Reduction in salt scaffold lengths from preshrinking and from the BVM cultivation. The * indicates a difference of change in length compared to the BVM cultivation group $(p<0.01)$.

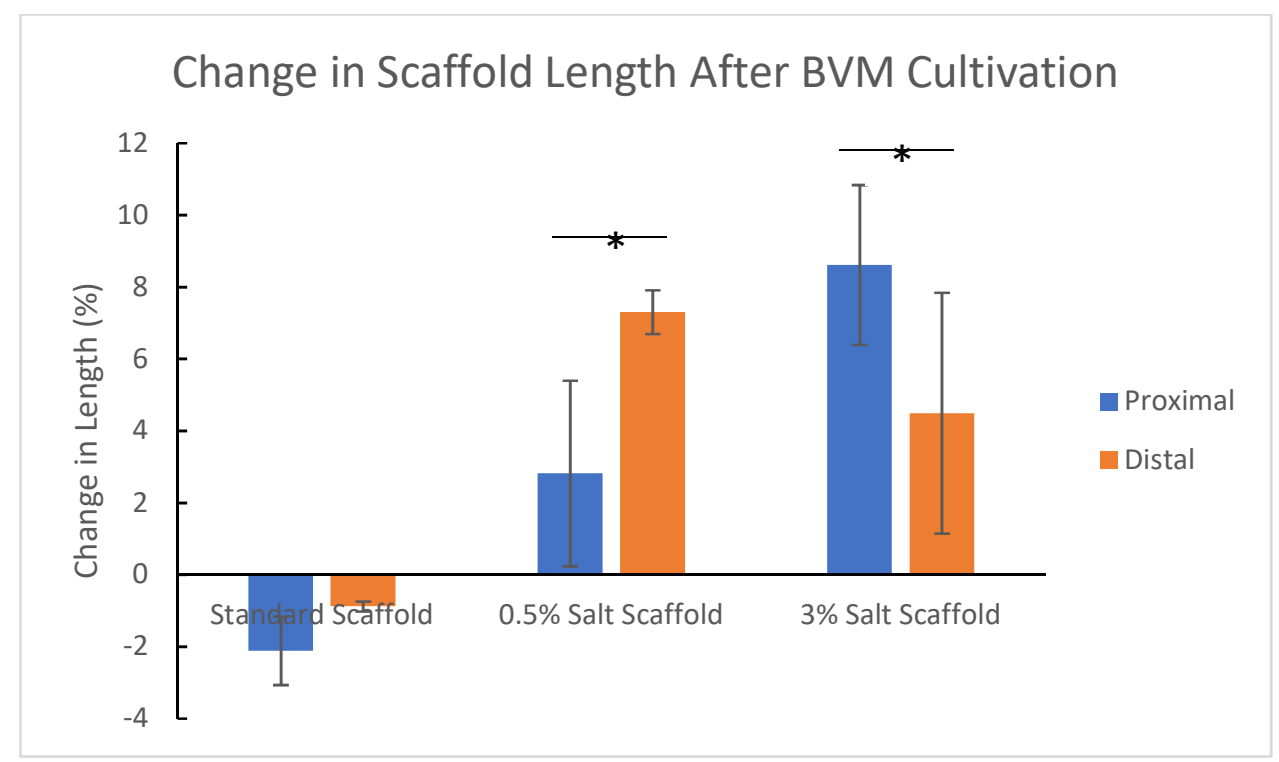

Figure 59: Standard and Salt Scaffold Shortening After BVM Cultivation. Reduction in the scaffold length during the 3 or 7-day BVM cultivation. The * indicates a difference for change in scaffold length compared to the standard scaffold group $(\mathrm{p}<0.05)$.

The thickness of the scaffold walls was measured after BVM cultivation. There was no difference in scaffold thickness between the $0.5 \%$ and $3 \%$ salt scaffold groups, 
but the 3\% salt scaffold group had a greater wall thickness than the standard scaffold group. This difference was expected; the salt scaffolds used $25 \%$ more PLGA than the standard scaffolds to accommodate for the $4 \mathrm{~mm}$ mandrel.

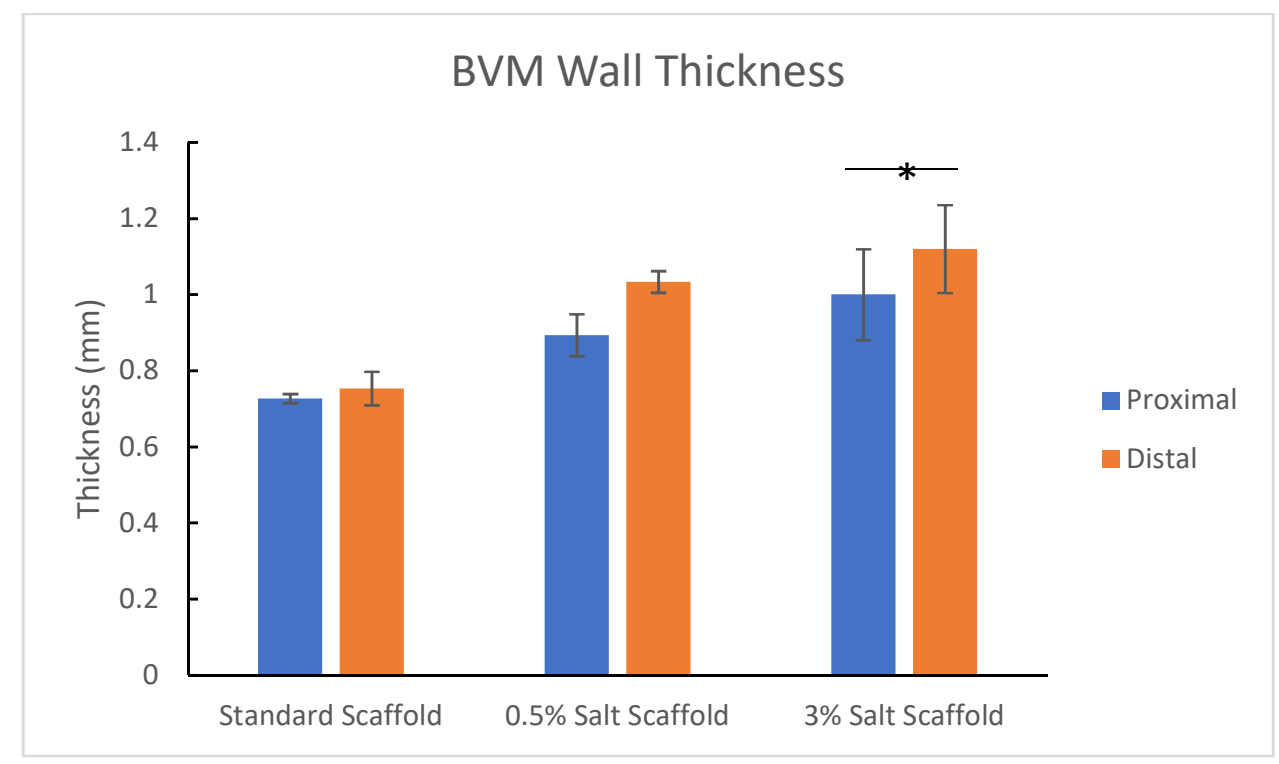

Figure 60: Wall Thicknesses of Scaffolds in BVMs. The * indicates a difference in thickness compared to the standard scaffold group $(\mathrm{p}<0.05)$.

Like with the cell adhesion assay, cell coverage was first quantified for the BVMs to investigate the effect of scaffold type and cultivation duration. Figure 61 shows images of 3-day BVMs from each scaffold group, and Figure 62 shows 7-day BVM images from each scaffold group. 


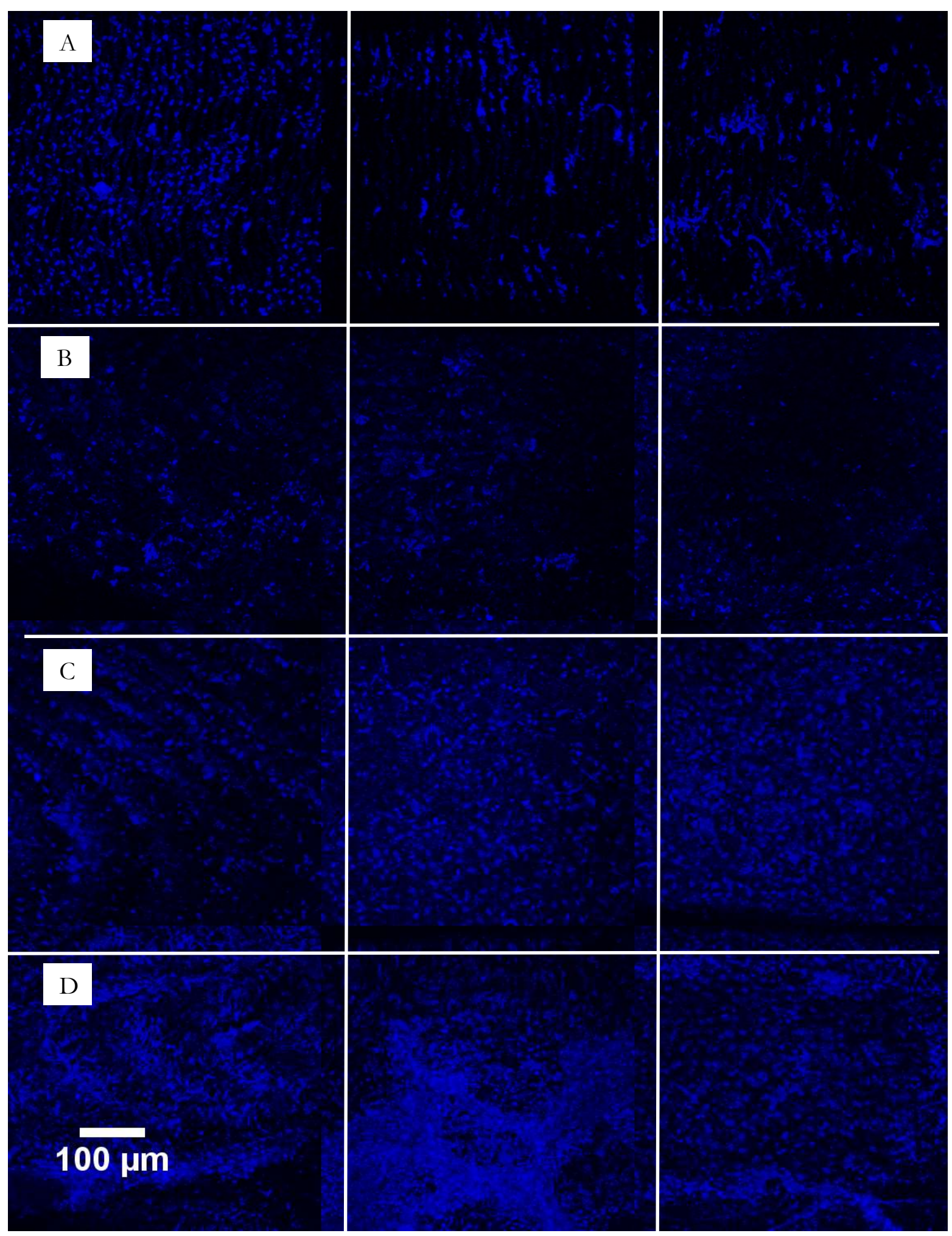

Figure 61: Representative 3-day BVM Hoechst Images. I'roximal (left), middle (middle), and distal (right) locations. (A) ePTFE scaffold, (B) standards scaffold, (C) $0.5 \%$ salt scaffold, (D) 3\% salt scaffold. High resolution images can be obtained from Dr. Cardinal. 


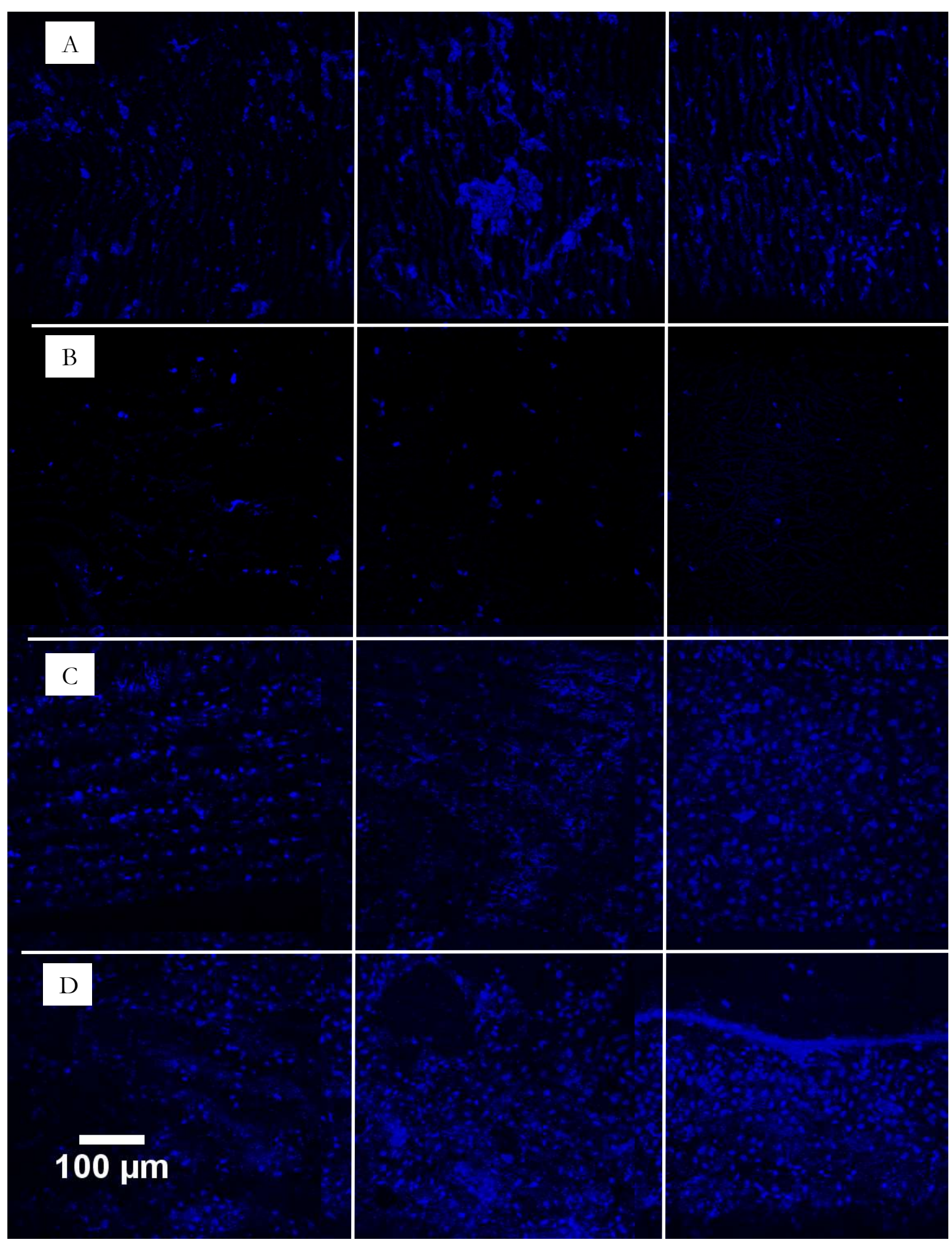

Figure 62: Representative 7-day BVM Hoechst Images. F'roximal (left), middle (middle), and distal (right) locations. (A) ePTFE scaffold, (B) standards scaffold, (C) $0.5 \%$ salt scaffold, (D) $3 \%$ salt scaffold. High resolution images can be obtained from Dr. Cardinal. 
The quantification results of BVM cell adhesion images are shown in Figure 63.

No differences between image locations (proximal, middle, distal) were detected, so these images were averaged in the values seen in this figure. The $0.5 \%$ and $3 \%$ salt scaffold BVMs had greater cell coverage than the ePTFE and standard scaffold BVMs. Every scaffold type had a difference in coverage between the 3 and 7-day timepoints; however, the 7-day vessels had less coverage than the 3-day vessels for all scaffold types. It would be expected that the longer time point vessels would have a better cell coverage than the shorter time point.

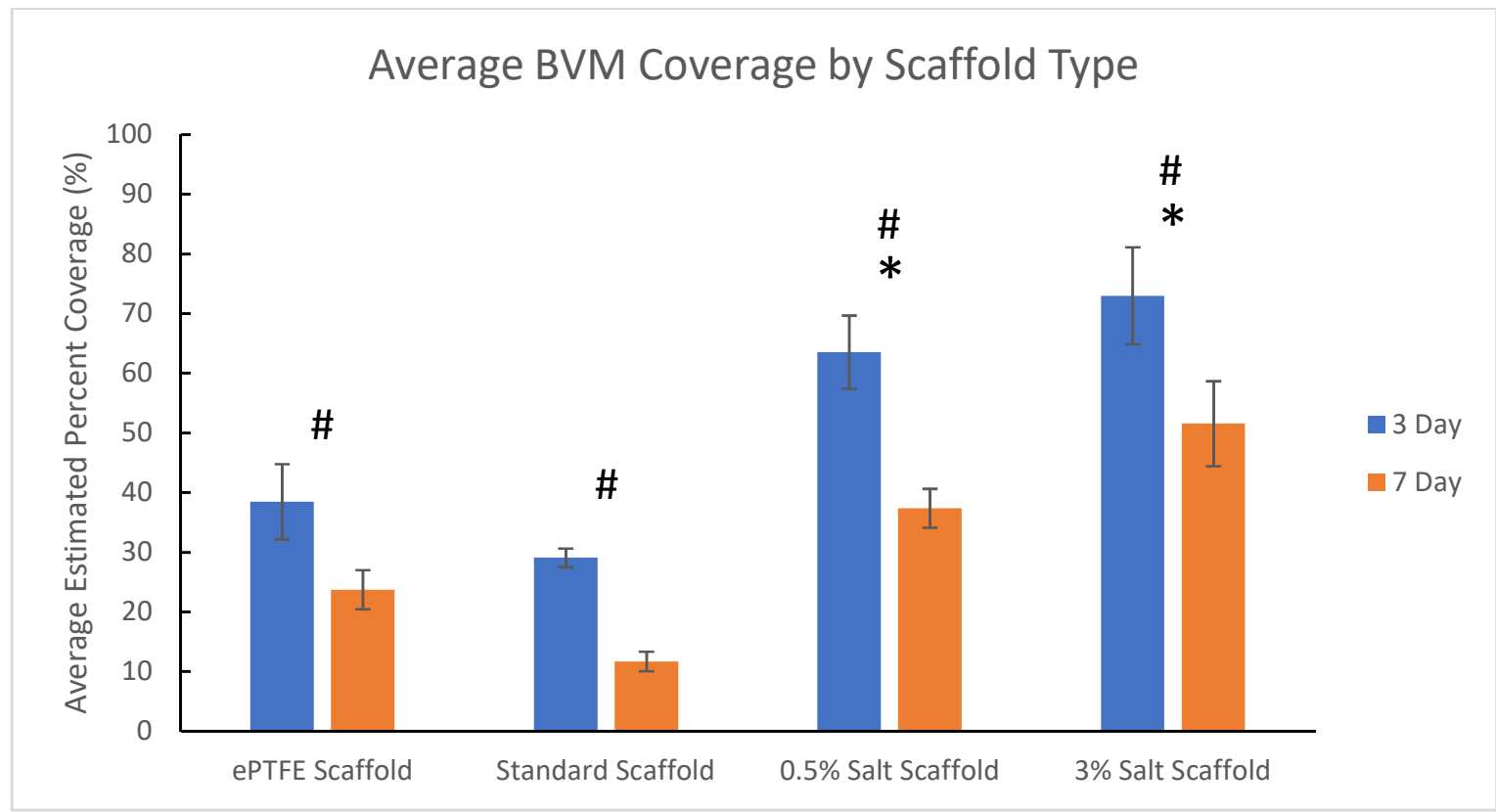

Figure 63: BVM Cell Coverage in 3 and 7-Day BVMs. The * indicates a difference in 3-day cell coverage compared to the 3-day ePTFE group and a difference 7-day cell coverage compared to the 7 -day ePTFE group $(\mathrm{p}<0.05)$. The \# indicates a difference in cell coverage between timepoints within the scaffold type $(\mathrm{p}<0.05)$.

Cell viability was also of interest, which was determined through Caspase and Propidium Iodide activity. Figure 64 and Figure 65 show representative images of the Caspase and Propidium Iodide channels from every BVM type at each time point. The 
quantification results of these images are shown in terms of estimated percent apoptotic and dead cells, as the Caspase and Propidium Iodide coverage was normalized to the cell coverage. The standard and 3\% salt scaffold BVM groups at 3 and 7 days, as well as the 7-day time point of the $0.5 \%$ salt scaffold BVM group, had a higher percent of apoptotic cells than the ePTFE BVM group, shown in Figure 66 . The $0.5 \%$ salt scaffold BVM was the only treatment group to show a difference between the 3 and 7-day timepoints, in which the 7-day BVMs had a higher percentage of apoptotic cells than the 3-day BVMs. For this group the 3-day time point showed levels of apoptosis similar to that of the ePTFE BVMs. Estimated dead cells did not reflect this pattern. 


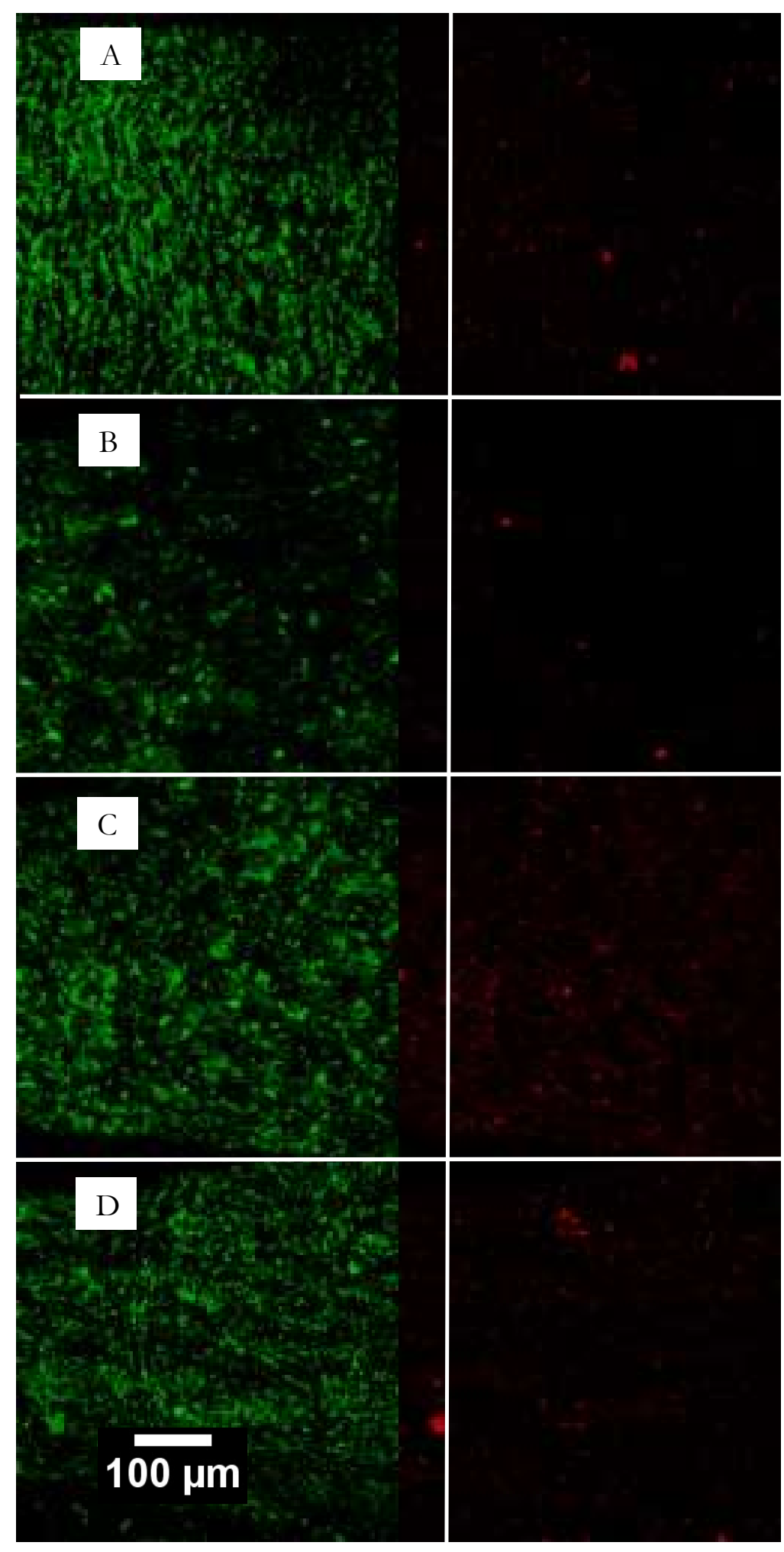

Figure 64: Images of 3-day BVM Caspase and Propidium Iodide Channels. Caspase channel (left) and Propidium Iodide channel (right) for all BVM types: (A) ePTFE scaffold, (B) standards scaffold, (C) $0.5 \%$ salt scaffold, (D) $3 \%$ salt scaffold. High resolution images can be obtained from Dr. Cardinal. 


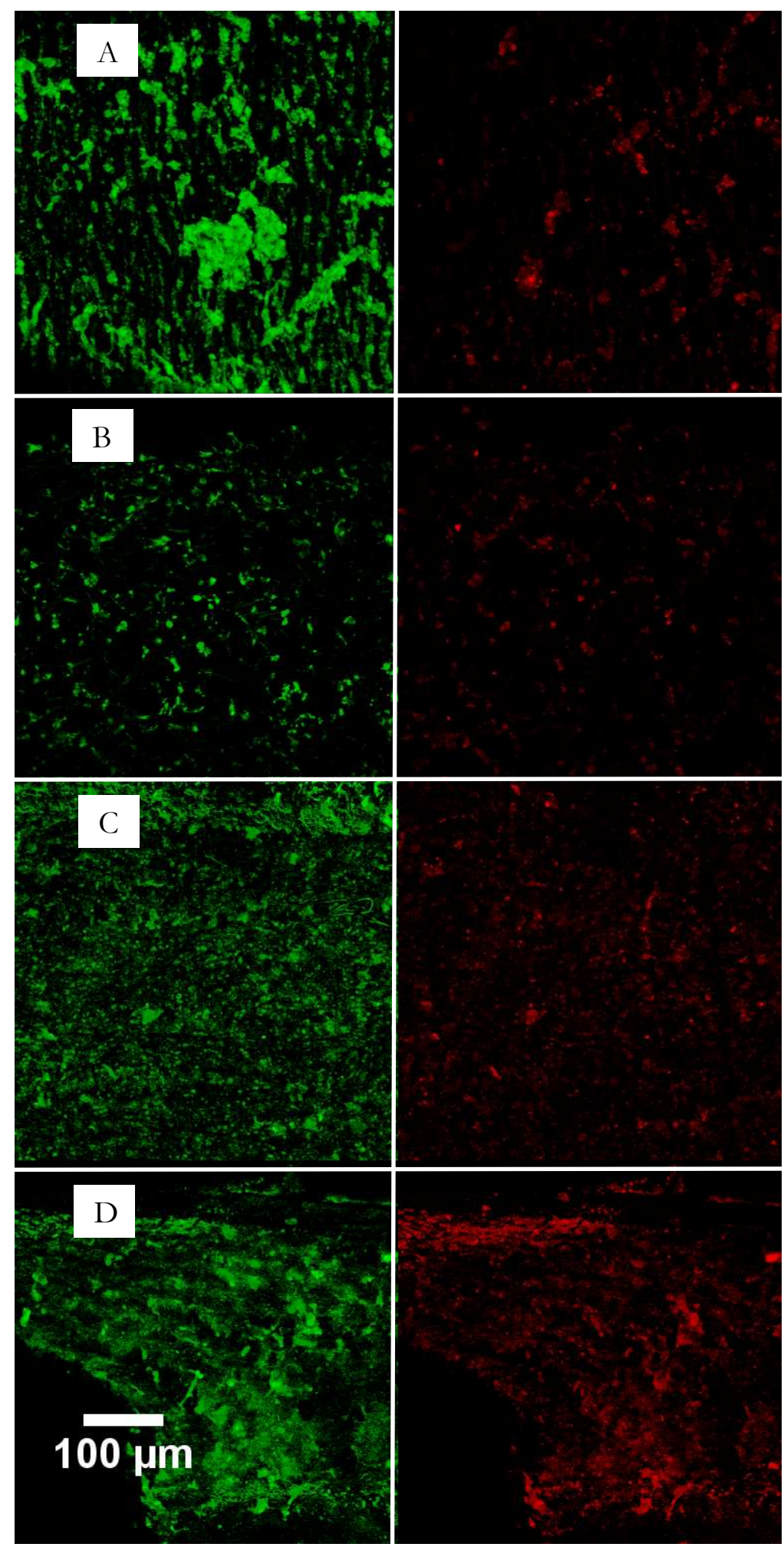

Figure 65: Images of 7-day BVM Caspase and Propidium Iodide Channels. Caspase channel (left) and Propidium Iodide channel (right) for all BVM types: (A) ePTFE scaffold, (B) standards scaffold, (C) $0.5 \%$ salt scaffold, (D) 3\% salt scaffold. High resolution images can be obtained from Dr. Cardinal. 


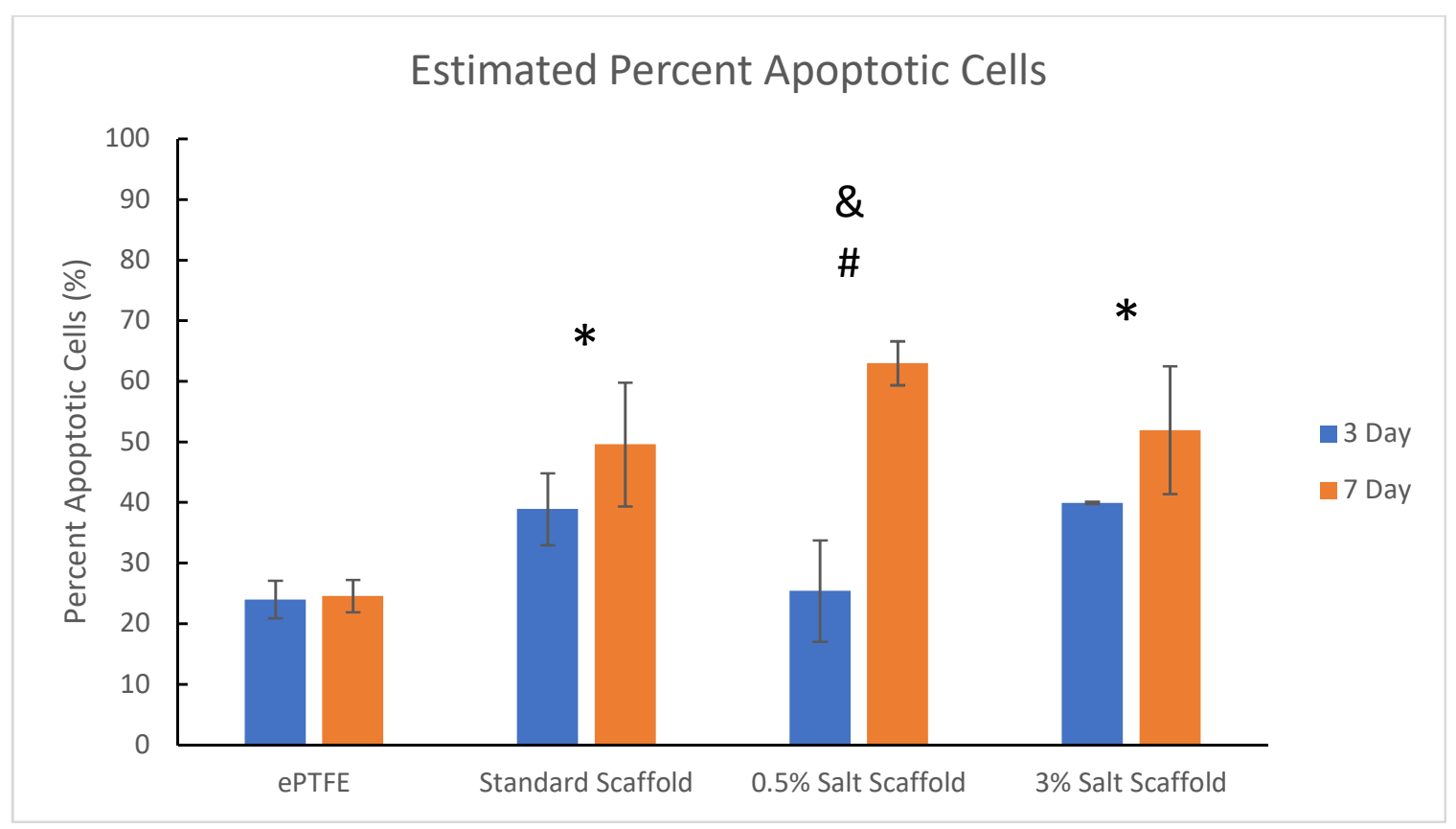

Figure 66: Percent Apoptotic Cells in 3 and 7-Day BVMs. The * indicates a difference in 3-day apoptosis percentage compared to the 3-day ePTFE group and a difference 7-day apoptosis percentage compared to the 7 -day ePTFE group $(\mathrm{p}<0.05)$. The $\&$ indicates a difference in apoptosis percentage from the ePTFE group compared only the 7-day time point $(\mathrm{p}<0.05)$. The \# indicates a difference in percent apoptosis between timepoints within the scaffold type $(\mathrm{p}<0.01)$.

Figure 67 shows that there were no differences in cell death between BVM types at 3 days, and at 7 days only the 3\% salt group was higher than the other three groups. 


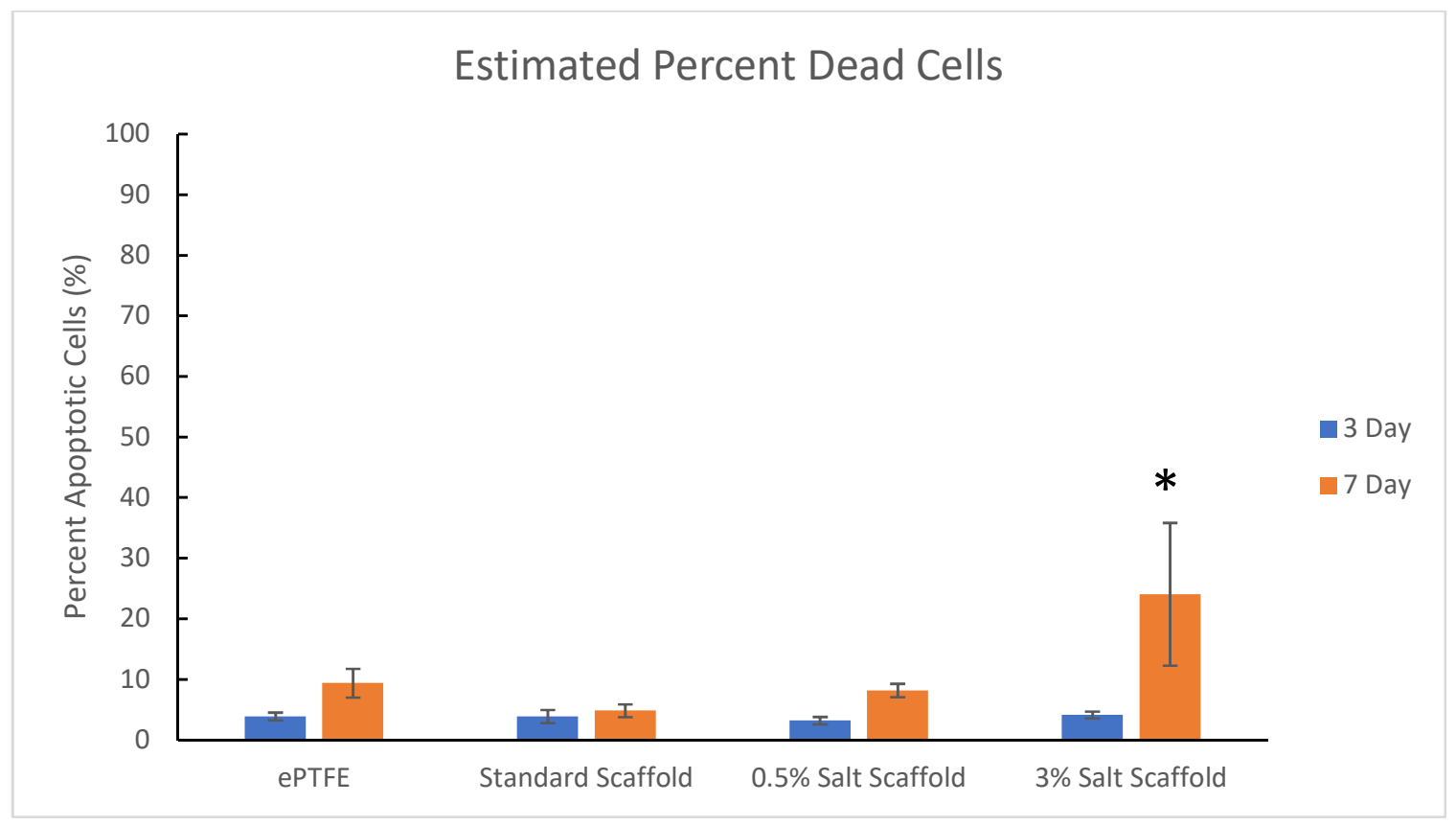

Figure 67: Percent Dead Cells in 3 and 7-Day BVMs. The * indicates a difference when compared to all other timepoints and scaffold types $(p<0.01)$.

\subsection{Discussion and Conclusion}

The purpose of Aim 2 was to establish methods with which to assess novel scaffolds through the cell-scaffold interactions. The primary measurements by which to achieve this were estimates of cell coverage and cell viability through measuring apoptotic and dead cells. It should be noted that it is possible for cells to be positive for both Caspase and Propidium Iodide ${ }^{170}$.

\subsubsection{Cytotoxicity Elution Assay}

The cytotoxicity assay treated cells with diluted or undiluted media that had been eluted with standard or salt scaffolds. Eluted media was applied for 24 and 48-hour timepoints. This study found that the $3 \%$ salt treated groups resulted in more apoptotic 
cells than the standard scaffold treated groups, either diluted or undiluted, until being normalized to the negative control. Once this data was normalized, this difference was no longer significant. This displays the importance of including appropriate controls within experiments; without removing the natural amount of apoptosis, incorrect conclusions could be drawn. The knowledge that the salt scaffold elutions were comparable to standard scaffolds in inducing apoptosis will be important when considering whether salt scaffolds should be used in BVMs.

After normalization, more dead cells were present at the 48 -hour time point than the 24-hour time point for all treatment groups except the no dilution standard scaffold group. An unexpected trend was found between the diluted and undiluted treatments. It would be expected that the undiluted treatments would result in more dead cells because there are more cytotoxic substances than in the dilution group. However, for both types of scaffolds the dilution groups resulted in more Propidium Iodide positive cells than the undiluted treatments. This disconnect between how the elutions are affecting the cells implies an unknown variable affecting cell death.

While conclusions regarding the effectiveness of the dilution groups cannot be drawn, it can be concluded that undiluted eluted media did not cause extensive levels of apoptosis or cell death. The dilution groups were a preemptive measure. If the undiluted elutions, regardless of the scaffold type, were too cytotoxic to the cells and killed all treatment groups, the resulting data would have been largely inconclusive. Since the undiluted treatments did not result in high amounts of apoptosis and dead cells, the dilution groups would serve no purpose in future studies and should therefore not be 
included. A control group of cell media heated at the elution settings should be added to future work to ensure that the elution process is not affecting the media characteristics.

Overall, this assay was useful in determining differences in cytotoxicity between scaffolds. It confirmed the results seen in the cytotoxicity study by Park et al in which a small planktonic crustacean was treated with BTEAC scaffold elution product and found to be unaffected ${ }^{164}$. Since the scaffolds were not cytotoxic in this assay, further experiments were carried out to address direct scaffold cytotoxicity and cell adhesion.

\subsubsection{Cell Adhesion and Viability Assay}

Theoretically, the nanofibers of the salt scaffolds should improve cell adhesion compared to the microfibers of the standard scaffolds ${ }^{128}$. The present work found that the $3 \%$ salt scaffolds had higher cell coverage than the standard scaffolds. Two to three times the number of cells were adhered to the salt scaffolds, even though half the number of cells had been initially sodded as compared to the standard scaffold. On top of that, a far greater percentage of those sodded cells were collected from the petri dish in which the scaffolds had been sodded. This speaks to the sodding efficiency of these scaffolds and implies that perhaps only $1.58 \mathrm{E} 6$ cells $/ \mathrm{mL}$, or about $6.7 \mathrm{E} 5 \mathrm{cells} / \mathrm{cm}^{2}$, would have been needed.

Another important observation was the resistance of the salt scaffolds during pressure sodding. The reason that less cells were sodded on the salt scaffolds as compared with the standard scaffolds was that more cell solution could not be physically forced through the salt scaffolds. It is possible that the cells quickly clogged the small pores in the salt scaffolds, blocking additional cells from being sodded. The standard 
scaffolds did not react in this way; little resistance was felt during pressure sodding. One explanation for this is that the cells were either passing through the standard scaffold pores or were populating within the walls of the scaffolds. The latter is more likely, as a low percentage of the sodded cells was collected from the petri dish, indicating that the cells remained in the scaffold. This could be verified through hematoxylin and eosin staining of cross-sections of scaffold wall to determine cell infiltration.

The estimated percent of apoptotic cells in the $3 \%$ salt scaffold group was not found to be different than that of the standard scaffolds, continuing to support the utility of salt scaffolds as replacements for standard scaffolds. Even though the percent of apoptotic cells did not differ, the percent of dead cells did, with the salt scaffolds having a higher percentage of dead cells than standard scaffolds. Since this difference was not seen regarding apoptosis, one of two explanations could explain this data. Either cells are dying as a result of pathways outside of apoptosis, or the methods used to remove background noise from the Propidium Iodide channel were insufficient. The second option is more likely than the first because it is unlikely that many cells are dying as a result of necrosis instead of apoptosis. The Propidium Iodide channel had a high amount of background noise in the images, so pre-quantification alterations should be revisited during image analysis of future studies.

\subsubsection{BVM Cell Coverage and Viability Assay}

Since the salt scaffolds improved cell adhesion without affecting apoptosis in the previous two studies, the scaffolds were tested for efficacy in BVMs. The salt scaffolds required preshrinking, and this method was shown to be effective at eliminating 
shrinkage before conditioning scaffolds in the bioreactors. Aside from this step, the salt scaffolds did not react differently than standard scaffolds when preparing them for or securing them in bioreactors. In fact, the salt scaffolds were often easier to secure to fittings because the material was more elastic than standard scaffolds, so the scaffold ends could be widened more easily without risk of tearing the scaffolds.

Cell coverage was greater in the $0.5 \%$ and $3 \%$ salt scaffold BVMs than the standard scaffold, which reflects the findings of the cell adhesion study. Cell adhesion between the two types of salt scaffolds was comparable. Regarding scaffold effects on apoptosis in BVMs, standard and 3\% salt scaffold BVMs at both timepoints had a higher percent of apoptotic cells than the ePTFE BVM. The 7-day time point for the $0.5 \%$ salt scaffold also had this difference from the ePTFE scaffold, but the 3-day time point did not. The $0.5 \%$ salt scaffold was the only group to have a difference in apoptosis between timepoints. The $0.5 \%$ salt scaffold at 7 days only had a single repetition (as a result of contamination), so this difference may not present itself with increasing repetitions of 7day BVMs. This difference did not translate to estimated dead cells. Only the 7-day 3\% salt scaffold had a higher percentage of dead cells than any of the other treatment groups. The $0.5 \%$ salt group was comparable to the standard scaffold group at both timepoints.

While these findings support the utility of the salt scaffolds in BVMs, there was one surprising trend in cell coverage. Each treatment group had lower coverage at the 7day time point than the 3-day time point. Since apoptosis did not increase between timepoints (except for the $0.5 \%$ salt scaffold BVM), scaffold cytotoxicity over time is unlikely to be the cause of this decreased coverage. Since this trend is consistent, it is possible that user error was a result. Since the two timepoints were not started on the 
same day and not sodded with the same cell solution, variability in the number of sodded cells and how the cells were sodded on that day may exist. Since the same concentration of cells was used on both timepoints, differences in sodding technique each day may be the cause. To eliminate this possible variability in the future, sets of BVMs should include vessels of both timepoints.

This study also dispelled concerns about salt scaffold cytotoxicity through direct contact. Park et al. documented the use of electrospun BTEAC salt scaffolds as antiviral and antibacterial surfaces ${ }^{161}$. This antiviral and antibacterial characteristic could negatively affect cells in direct contact with the scaffold. However, the work in this thesis did not show that high or low concentrations of BTEAC salt scaffolds caused more cytotoxicity than standard PLGA scaffolds. Furthermore, even though these scaffolds were shown to be antibacterial by Park et al., a $0.5 \%$ salt scaffold did become contaminated with what was most likely bacteria. The contamination of this $0.5 \%$ salt scaffold BVM was unlike past contaminations of standard scaffold BVMs. Usually, the bioreactor and reservoir media changes color and becomes cloudy. However, this BVM only showed this color change in the reservoir and not in the bioreactor. This indicates that the salt scaffold contained the contamination to only the flow loop from the lumen of the scaffold to the bioreactor. If this is the case, then this suggests that salt scaffolds may allow little perfusion of media through the wall of the scaffolds.

\subsubsection{Limitations and Future Work}

A substantial limitation in this aim was the use of a poly-Caspase stain, which stained many types of Caspase in the cell. While this stain was useful for initial 
investigations, it may have been nonspecific. Other stains are available which only stain for Caspase that are active in the execution pathway of the apoptosis cascade. Future work should investigate and, most likely, implement a more specific Caspase stain, such as the Image-IT LIVE Green Caspase-3 and 7 Detection Kit (Molecular Probes). This would only label cells in the execution pathway which will apoptose, unlike with other Caspases that may be active even though the cell will save itself from apoptosis.

The quantification method for analyzing cells on scaffolds was limited by several assumptions. The first assumption was the factor used to multiply the nucleus area to estimate the cell coverage. This is not necessarily an accurate estimate because it does not reflect coverage in areas where cells are clumped. Clumped cells reflect a higher cell coverage than should be represented. This factor, while limiting, does not change the trends seen by the data because the value is constant. Since it was possible for this factor to be incorrect, conclusions were not made that mentioned specific coverage percentages. Another concern was the possibility that the Caspase antibody stained both active caspase and procaspases. The fluorochrome-labeled inhibitors of caspase (FLICA) method of labelling, which was utilized by the Caspase stain in these studies, binds the active centers of caspases with a fluoromethyl ketone $(\mathrm{FMK})^{171}$. The active recognition sequence on which FMK binds is valine-alanine-aspartic acid (VAD); this active site should only be available in active caspases in which the small subunit and large subunit of the procaspase bind. Procaspase to not have this active site ${ }^{170,171}$. However, a procaspase 3 monoclonal antibody (ThermoFisher Scientific) could be compared against an active caspase 3 immunostain (Abcam) to determine if this staining mechanism is nonspecific. Active caspase 3 should only be present in the execution phase of apoptosis, 
while procaspase 3 should be present in non-apoptotic and apoptotic cells. Therefore, procaspase would be visible in healthy cells, while a high abundance of active caspase would only be present after an apoptosis-inducing treatment.

The BVM cell coverage and viability assay showed some cell clumping, which was not observed in the short-term cell adhesion and viability assay of cells on scaffolds in static media. The concern with this is that these clumps do not display standard endothelial morphology and have some areas that look like scaffold stained with antibodies. It was verified that salt and standard scaffolds do not autofluorescence using the Hoechst, Caspase, and Propidium Iodide stains, and that these cell clumps contained individual nuclei in the Hoechst channel. Despite this, future work should make note of cell clumping when working with the BTEAC salt scaffolds to ensure that cell clumping does not present as a continued issue.

These three studies did not contain enough repetitions to be statistically significant, which limits the conclusions drawn in this aim. The cost and labor associated with creating BVMs generally make a statistically designed study less plausible. However, power analyses of cell coverage, percent apoptotic cells, and percent dead cells of cell coverage for a power of 0.80 found that five repetitions per treatment is necessary for cell coverage and percent apoptotic cells, while six repetitions per treatment are necessary for percent dead cells. It would be possible to conduct an experiment with 48 vessels, but would require a huge effort by the Tissue Engineering Lab. It is, however, more plausible to design experiments for the cytotoxicity elution assay and short-term cell adhesion assay that include enough repetitions of each treatment group to be statistically significant. Power assessments found that 6 scaffolds of each type are 
necessary for a statistically designed cytotoxicity elution study, while 3 scaffolds of each type are necessary for a short-term adhesion study. Future studies of scaffold cytotoxicity and cell adhesion should be planned and executed for both assays to draw conclusions backed by statistical repetitions.

Modifications could be made to the elution protocol in future studies by concentrating the eluted media. This would allow for control of the intensity of the media in which is being delivered to cells. Using a more concentrated product may make evident trends that could not be identified with less concentrated eluted media. Concentration of the media could be achieved through filtration using the Ultrafree 15 (Sigma-Aldrich) or through phenol extraction ${ }^{172}$.

Other experiments and analysis could be conducted to investigate the use of these scaffolds in BVMs. BVM cross-sections could be stained to compare and determine differences in cell penetration into the scaffold walls. Cryosectioning scaffolds and staining with hematoxylin and eosin would provide this information.

Since the purpose of the BVM model is to test vascular devices, scaffold types should eventually be compared for sue as BVMs with stents deployed within them. If HUVEC-only BVMs using salt scaffolds displayed similar efficacy to dual-sodded standard scaffolds with stents deployed in both groups, the salt scaffolds would be superior due to the cell sodding efficiency and simplicity of EC-only BVMs and could be implemented for future BVM studies. 


\subsubsection{Summary}

This aim was successful in implementing methods and comparing scaffold cytotoxicity and cell adhesion between BTEAC salt and standard PLGA scaffolds. No differences were seen in apoptotic activity between the 3\% salt and standard scaffolds in the cytotoxicity elution assay or short-term adhesion assay. This trend continued between the $3 \%$ salt and standard scaffolds in BVMs, while the $0.5 \%$ scaffold had less activity at the shorter time point but caught up by the 7-day time point. Cell coverage was superior in the salt scaffolds. With this knowledge, it can be concluded that the salt scaffolds are comparable to the standard scaffolds regarding cytotoxicity but superior in cell adhesion and coverage. However, further comparisons of the $0.5 \%$ and $3 \%$ salt scaffolds still must be conducted. This conclusion may rely upon some physical evaluations of $0.5 \%$ and $3 \%$ salt scaffolds, such as yield strength and fiber diameter. Regardless, testing the interaction of cells on electrospun BTEAC salt scaffolds is novel, as it has not been previously documented in published literature. The results in this aim support the conclusion that standard, $0.5 \%$ salt, and $3 \%$ salt scaffolds have similar cell viability, as assessed through Caspase and Propidium Iodide activity, and salt scaffolds have higher cell adhesion and coverage than standard scaffolds. 


\section{Chapter 4: DISCUSSION AND CONCLUSION}

\subsection{Summary and Aims of the Thesis}

BVMs have proven useful as a preclinical model for testing vascular devices by providing initial data on how the device interacts with cells ${ }^{4,9}$. This success warrants further investigation into the individual components of the model. Improvements to these components must be justified and reinforced before implementing them as standard procedures. Therefore, after identifying an area of improvement, methods must be established to measure changes in component responses.

The overall goal of this thesis was to establish methods to characterize the endothelial cells used in the BVM model. The endothelial cell component is, arguably, the most important part of the model since the EC interaction with vascular devices is the most important measured response ${ }^{173}$. Two aspects of EC involvement were identified which could be improved but lacked established methods to support any changes. Aim 1 established methods to compare EC gene expression at different passages and in different flow conditions in the context of studying EC dedifferentiation. The use of ECs that respond like endothelial cells and appropriately express endothelial markers is necessary to claim that the cell interaction with the vascular device is reflective of an interaction that would occur in vivo. Aim 2 established methods to compare standard PLGA scaffolds to BTEAC salt-PLGA scaffolds through studying scaffold cytotoxicity on ECs and EC cell coverage at varying lengths of time. These methods and the subsequent results contributed to the model in two ways. The Tissue Engineering lab had no previous methods for implementing new scaffolds despite having investigated and designed other 
types of scaffolds in the past, so this aim established methods that can be used for future scaffolds. This aim also identified and tested a novel scaffold that could be implemented as an improved scaffold for creating BVMs.

\subsection{Results and Discussion}

Aim 1 can be divided into two subsections: changes in EC gene expression over culture time, and changes in EC gene expression as a result of various flow conditions. EndMT would result in downregulation of endothelial markers and upregulation of mesenchymal markers, while ECs which retain their phenotype would increase expression of VEGFR2 and VE-cad through VEGF signaling. In the first set of experiments, a trend was identified of increasing expression of the endothelial markers VE-cadherin and VEGFR2 as culture time increased. The EC marker PECAM, as well as the mesenchymal markers ACTA2 and Calponin, remained stable as culture time increased. These trends do not support culture time contributing to EC dedifferentiation; EC dedifferentiation would result in loss of EC markers and an increase in mesenchymal markers.

The second set of experiments in this aim studied gene expression changes in ECs cultured in static, disturbed, or laminar flow conditions. The establishment of a disturbed flow condition was successful in that it showed a decrease in VE-cadherin and VEGFR2 abundance compared to ECs in static culture. Unfortunately, the disturbed flow condition had to be removed from the analysis. The dissociation curves for this group showed untargeted amplification during qPCR, indicating contaminants within the RNA or cDNA. However, the static culture group was still compared to the laminar flow group of 
the BVMs. This found that the laminar flow group had lower expression of all three endothelial markers and ACTA2 than the static culture group, which contradicts trends seen in literature. These findings are likely explained by the 24-hour time point used for the BVM, which may have not allowed enough time for ECs to re-establish the cell-cell junctions measured by the EC markers.

The investigations in Aim 1 were largely inconclusive, although the methods established can be applied to ongoing and future work. Some trends were identified, but they did not provide evidence of EC dedifferentiation occurring. These trends are positive for the BVM model, as they suggest cells retaining an EC phenotype. However, all studies in this Aim were investigational for method development and not statistically powered. Properly powered studies would have been conducted had the initial data suggested that further studies would provide useful data. Unfortunately, this was not the case, so further studies were not pursued. Despite this, the methods developed here may prove useful in future studies, which will be discussed in Section 4.4, so gene expression analysis through qPCR should continue to be implemented as an analysis technique by the Tissue Engineering Lab.

Aim 2 was composed of three parts corresponding to each of the three assays developed. In the first part, the cytotoxicity elution assay treated ECs with scaffold-eluted media for 24 or 48 hours. No differences were found in apoptosis between scaffold type, and more dead cells were found in the 48-hour group regardless of scaffold type. Since the change in dead cells was not a factor of scaffold type and apoptotic activity was not different between scaffold types, it was concluded that salt scaffolds did not adversely 
affect cell viability. This elution assay did not test viability through direct cell contact, but the two other assays did.

In the second part, the cell adhesion and viability assay looked at initial cell deposition and apoptotic activity at a 4-hour timepoint. The salt scaffold did not differ in Caspase activity compared to standard scaffolds, but cell death was higher in the salt scaffold group than the standard scaffold group. Cell adhesion was higher for salt scaffolds than standard scaffolds. This suggested that, while more HUVECs adhered at a short timepoint, the cells may not persist as time increased.

The final part of this aim, the BVM cell coverage and viability assay, was similar to the previous assay but compared scaffold types in BVMs over multiple days. Cell coverage was higher in the $0.5 \%$ and $3 \%$ salt scaffolds than standard scaffolds regardless of time point. Apoptosis was not higher in the salt scaffolds than the standard scaffolds. This was also true for cell death, except in the 7-day 3\% salt scaffold group which had more cell death than any of the other scaffold types at that time point. These findings suggested that HUVECs had similar rates of survival but better coverage in BTEAC salt scaffolds than standard PLGA scaffolds.

These three parts of Aim 2 found that apoptosis did not differ between standard PLGA scaffolds and salt scaffolds, but cell adhesion and coverage was notably improved for salt scaffolds. The only adverse effect of the salt scaffolds was regarding cell death. This may have been due to the quantification method because it would be expected that apoptotic activity would reflect the trends of cell death, which was not seen. The form in which cells died may have also contributed to this result; if cells died through necrosis, the apoptosis pathway and caspases would not have been activated. A comparison of 
apoptosis to necrosis could be evaluated through immunofluorescence using an Apoptosis/Necrosis Assay Kit (Abcam), which identifies all cells, apoptotic cells, and necrotic cells. Nonetheless, it can be concluded from this data that the salt scaffolds are superior to the standard scaffolds. The $0.5 \%$ salt scaffold is recommended as the scaffold candidate to pursue if the comparison of fiber diameter and other physical characteristics in the $0.5 \%$ and $3 \%$ salt scaffolds do not reveal significant differences.

\subsection{Challenges and Limitations}

The greatest limitation of both Aim 1 and Aim 2 was the lack of statistical significance in any of the studies. Since both aims were investigational and intended for method development, the experiments were not designed with statistical power. The intent of these studies was to properly design experiments if the initial pilots revealed promising information. For Aim 1, this was not the case, which lead to no statistically powered studies being conducted. For Aim 2, the pilot studies did reveal promising information that warranted full studies to be conducted, which will be discussed in the next section.

A similar limitation has to do with designing properly powered experiments that use BVMs. The amount of resources and time necessary to produce a small amount of BVMs makes it difficult to design and complete properly powered experiments. In order to statistically power Aim 2, 48 BVMs would have been necessary. This is not a practical amount of BVMs to fabricate for a single study. Issues with contamination can further reduce the repetitions of treatment groups. Triplicates of each BVM treatment group is a feasible goal that could be used in place of a statistically powered study. 
The main challenges in Aim 1 were with the disturbed flow condition. In the second set of experiments, which compared the disturbed flow condition to a negative control (static EC media treated) and positive control (static SMC media treated), there was a high amount of cell death which yielded too low of RNA concentrations to run qPCR on the samples. As a result, only one of the disturbed flow passages could be compared with the other two treatments, so comparisons of expression change over culture time were lost. There were also issues with the disturbed flow treatment group in the third experiment comparing all three flow groups. A ramp up period was introduced in this study, which greatly reduced the cell death in the disturbed flow group and a high enough concentration of RNA was isolated to run qPCR. However, the dissociation curve for this group showed nonspecific targets in the qPCR products, so they were removed from the study. It was unclear at which step this issue occurred.

A main limitation of Aim 2 was having no SEM images of BVMs, as it was initially planned to include these images. This would have provided additional images from which to observe cell coverage. These images may have been able to visualize differences cell morphology on the scaffold types. Unfortunately, the SEM was broken, and images could not be taken in time to include in this thesis.

\subsection{Future Work}

Although this thesis served to establish methods to characterize endothelial cells for specific applications, these methods are generic and can be implemented for other studies. Many other applications of these methods may exist and will prove to be useful in future studies that have not yet been identified. These sections outline some 
applications that could be investigated in the future, and some applications that are already being investigated.

A study that links Aim 1 and Aim 2 would be an investigation of gene expression changes with different scaffold types. This could provide further data to support implementing salt scaffolds for general use by providing evidence of scaffold type promoting an endothelial phenotype or endothelial growth. Since calponin did not prove to be a useful gene in characterizing EC expression changes, that marker could be removed from the GOI and replaced by a proliferation marker like Ki67. This marker would indicate whether the sodded cells were quiescent, or whether the cells were proliferating in the BVM. The sodding density used within BVMs should not promote proliferation, but this marker would provide information to determine scaffold effects on proliferative potential.

Aim 1 has already proven effective at steering current work. The previous immunostaining protocol implemented in the Tissue Engineering Lab used PECAM and ACTA2 as markers to discern between ECs and SMCs, respectively. However, as previously established, HUVECs co-expressed PECAM and ACTA2. Through the gene expression studies in Aim 1, it was clear that Calponin was not expressed in HUVECs. However, Aim 1 did establish that Calponin was abundant in HUASMCs, making Calponin an excellent marker to replace ACTA2 for immunostaining. This recommendation was implemented, and no signal was observed in immunostaining for Calponin in HUVECs. The Calponin stain also proved to be a clear marker for immunostaining HUASMCs, shown in Figure 68. 


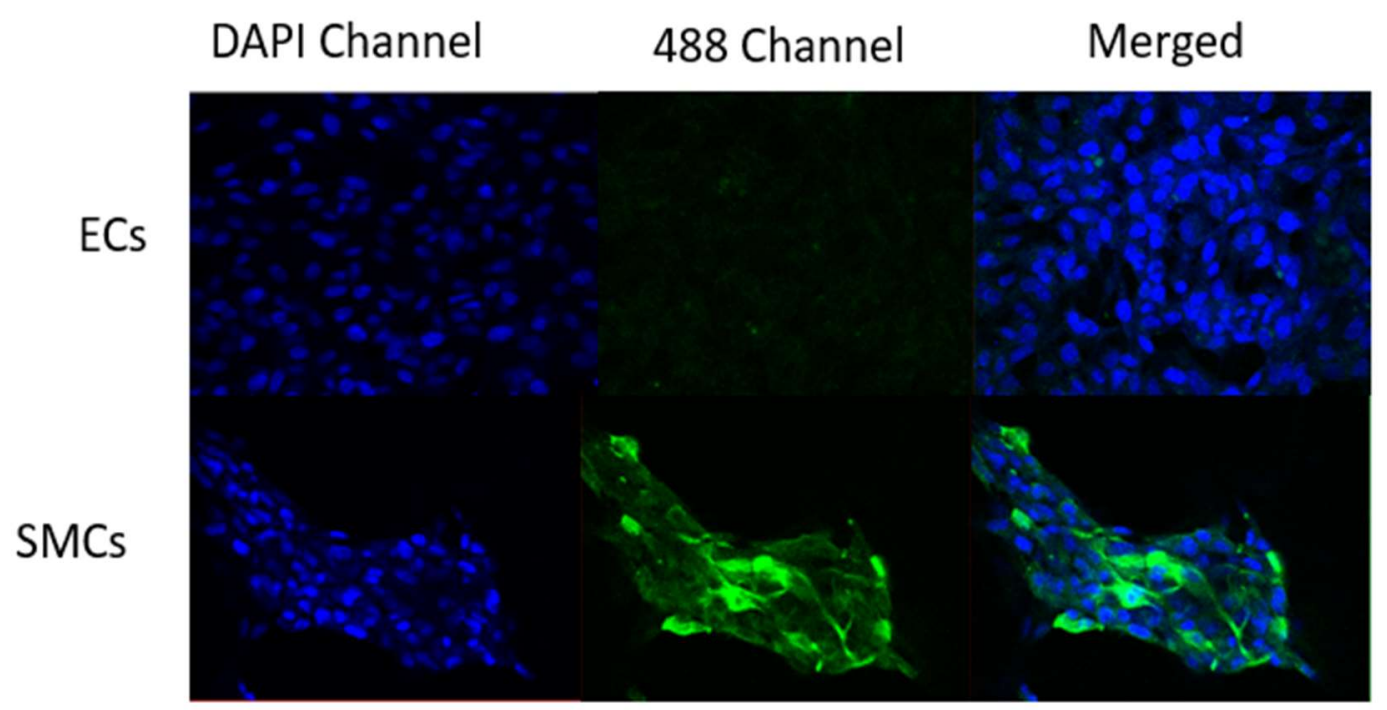

Figure 68: Calponin immunostaining of HUVECs and HUASMCs. No Calponin signal is seen in HUVECs (top row), while Calponin signal is abundant in HUASMCs (bottom row).

The three EC markers used in Aim 1 could also be applied for studies related to changing flow rates within BVMs. The current maximum pump rate used in BVMs is 90 RPMs, which translates to only $5.9 \mathrm{~mL}$ of media being pushed through BVMs each minute. Increasing this pump rate or increasing the viscosity of media would increase shear stress, which is currently far below physiologic levels. Aim 1 showed HUVECs survived under higher levels of shear stress in the disturbed flow condition, which had shear stress values ranging from 5 to $15 \mathrm{dyne} / \mathrm{cm}^{2}$. Changes to the pump rate may be reflected by changes in the mechanosensory complex genes.

Regarding Aim 2, and as mentioned in Section 4.3, a major limitation was not running statistically powered studies. The data from the cytotoxicity elution study and short time point adhesion study provided useful data regarding cytotoxicity and cell adhesion, so properly designed studies using both assays have been planned. The cytotoxicity study will include three time points $(24,48,72$ hours) and three scaffold 
types (standard, $0.5 \%$ salt, $3 \%$ salt), but will eliminate the dilution group. The 4-hour adhesion and viability assay will add the $0.5 \%$ salt scaffold group as well. These two experiments will continue to build upon conclusions made in Aim 2.

The cytotoxicity elution assay is also already being implemented for testing the cytotoxicity of aneurysm scaffolds. Wax is molded in the shape of the aneurysm on a mandrel before electrospinning the mandrel. After electrospinning, the wax is dissolved in a warm media solution to remove the wax. However, some wax remains in the lumen of the scaffold after dissolving. Multiple types of wax have been used to make these aneurysm geometries. An ongoing study uses the elution assay to determine whether wax remaining in the scaffold is cytotoxic, and whether there is a difference in toxicity between wax types.

Future process adjustments can utilize the cytotoxicity assay to support viability assessments. A possible application is switching from ethanol sterilization of scaffolds to ethylene oxide (EtO) sterilization. Concern of EtO gas residuals in the scaffolds has always precluded this prospect. Current methods for testing EtO residuals involve extraction in water, so the cytotoxicity elution assay would be an appropriate method to determine the effect of residuals on cell viability ${ }^{174}$. Most importantly, EtO sterilization would substantially simplify the BVM fabrication process; scaffolds could be secured in bioreactors before sterilization, so only media would need to be added to the bioreactors and the flow circuit assembled before starting conditioning. No sterile gloves would need to be used since the scaffolds would not need to be flushed or secured in the sterile bioreactor. The removal of these steps would considerably decrease the amount of time necessary to create BVMs. Currently, it takes about 10 hours to set up six BVMs. 
However, the removal of these steps would decrease that time to approximately five hours, which would allow the Tissue Engineering Lab to substantially increase the amount of BVMs produced. EtO sterilization is also a superior sterilization method. Ethanol sterilization is considered a chemical disinfectant, not a sterilization process, because certain viruses and bacterial spores are resistant to the effects of ethanol ${ }^{175}$. An experiment could be run which used the cytotoxicity elution assay or direct contact assays to determine how EtO sterilized scaffolds affect cell viability.

A discussion of the future use of salt scaffolds must also be had within the Tissue Engineering Lab. Based on the literature, nanofiber scaffolds will likely be advantageous to implement because of the superiority to microfibers, and the data in Aim 2 suggests that the BTEAC salt scaffolds are a viable option for fabricating nanofibers. The question is not if, but when, nanofiber scaffolds replace the current microfiber scaffolds. A discussion must be had of what further testing of these scaffolds needs to be completed to justify implementation. Some potential further testing includes mechanical testing of scaffold characteristics like yield strength, fiber diameter assessment in larger data sets to ensure consistent sized nanofibers, and deployment of intravascular devices within BVMs using these scaffolds to determine that these scaffolds are useful for obtaining safety data of intravascular devices. Logistics of salt scaffold use must be explored as well, such as whether one or two cell types will be used, and the appropriate sodding density. 


\subsection{Conclusion}

Aim 1 of this thesis established methods for evaluating endothelial cells and BVMs through gene expression analysis and results supported the conclusion that HUVECs used in BVMs are retaining an endothelial phenotype during culture and not dedifferentiating from EndMT. In fact, longer culture lengths trended towards increased expression of VEGFR2 and VE-cadherin, indicating a stronger endothelial phenotype. Disturbed flow conditions were established as a positive control for dedifferentiation, which decreased VEGFR2 and VE-cadherin expression. Gene expression comparisons of BVMs to static and disturbed flow cultures were inconclusive.

Aim 2 of this thesis successfully developed protocols to evaluate scaffold cytotoxicity, as well as cell viability and coverage. These assays were applied to novel BTEAC salt scaffolds to compare against standard PLGA scaffolds. These studies found that BTEAC salt scaffolds do not affect cytotoxicity and cell viability more than standard PLGA scaffolds. The BTEAC salt scaffolds also have more cell coverage than standard scaffolds at all timepoints. Therefore, the integration of BTEAC salt scaffolds into the BVM model should be considered, replacing the current scaffolds. 


\section{REFERENCES}

1. Center for Disease Control and Prevention. Heart Disease Facts. https://www.cdc.gov/heartdisease/facts.htm. Published 2017.

2. Souilhol C, Harmsen MC, Evans PC, Krenning G. Endothelial-mesenchymal transition in atherosclerosis. Cardiovasc Res. 2018;114(4):565-577. doi:10.1093/cvr/cvx253

3. Medtronic. TREATMENT OPTIONS FOR CORONARY ARTERY DISEASE

(CAD). https://www.medtronic.com/us-en/patients/treatments-therapies/heartsurgery-cad/treatment-options.html. Published 2017.

4. Cardinal K, Bonnema G, Hofer H, Barton J, Williams S. Tissue-engineered vascular grafts as in vitro blood vessel mimics for the evaluation of endothelialization of intravascular devices. Tissue Eng. 2006;12(12). doi:10.1089/ten.2006.12.3431

5. Rice University. Anatomy and Physiology.

https://opentextbc.ca/anatomyandphysiology/chapter/20-1-structure-and-functionof-blood-vessels/. Published 2018.

6. Lonza. Endothelial Cells and Functions. doi:10.3390/pharmaceutics6040557

7. Brown BA, Williams H, George SJ. Chapter Six - Evidence for the Involvement of Matrix-Degrading Metalloproteinases (MMPs) in Atherosclerosis. Prog Mol Biol Transl Sci. 2017;147:197-237.

8. Pearson Education. Structure and Function of Arteries, Capillaries, and Veins.

http://www.phschool.com/science/biology_place/biocoach/cardio2/structure.html.

9. Herting S, DiBartolomeo A, Pipes T, et al. Human Umbilical Versus Coronary Cell Sources for Tissue-Engineered Blood Vessel Mimics. Appl Vitr Toxicol. 2016;2(3):175-182. doi:10.1089/aivt.2016.0012 
10. Shen TW, Puccini B, Temnyk K, Herting S, Cardinal KO. Tissue-engineered aneurysm models for in vitro assessment of neurovascular devices. Neuroradiology. 2019;61(6):723-732. doi:10.1007/s00234-019-02197-x

11. Sugerman G. Evaluation of Endothelial Cell Responses to Elevated Glucose In Vitro. 2018;(August). http://e-journal.uajy.ac.id/14649/1/JURNAL.pdf.

12. Kunz SG. Development and Characterization of Tissue Engineered Blood Vessel Mimics Under "Diabetic" Conditions. 2017;(June).

13. Negro A, Fang F, Chen G, et al. TGF- $\beta$ s signaling mediates endothelial to mesenchymal transition (EndMT) during vein graft remodeling. Sci Transl Med. 2014;6(227):1-22. doi:10.1126/scitranslmed.3006927.TGF-

14. Tzima E, Irani-Tehrani M, Kiosses WB, et al. A mechanosensory complex that mediates the endothelial cell response to fluid shear stress. Nature. 2005;437(7057):426-431. doi:10.1038/nature03952

15. Zeisberg EM, Potenta SE, Sugimoto H, Zeisberg M, Kalluri R. Fibroblasts in Kidney Fibrosis Emerge via Endothelial-to-Mesenchymal Transition. 2008;19(12):22822287. doi:10.1681/ASN.2008050513

16. Zeisberg EM, Potenta S, Xie L, Zeisberg M, Kalluri R. Discovery of endothelial to mesenchymal transition as a source for carcinoma-associated fibroblasts. Cancer Res. 2007;67(21):10123-10128. doi:10.1158/0008-5472.CAN-07-3127

17. Nick A S, Martin A L. Real-Time PCR Quantification Analysis. Caister Acad Press. 2013;2:11-13. https://www.thermofisher.com/us/en/home/brands/thermoscientific/molecular-biology/molecular-biology-learning-center/molecularbiology-resource-library/spotlight-articles/basic-principles-rt-qpcr.html. 
18. McGuffick T. Optimizing a Quantitative Real-time polymerase Chain Reaction Protocol for the Characterization of Gene Expression in Blood Vessel Mimics. 2018;(June).

http://genomebiology.com/2002/3/7/research/0034.1\%5Cnhttp://genomebiology.c om/2002/3/7/research/0034.

19. Dowey E. Identifying and Reducing Variability, Improving Scaffold Morphology, and Investigating Alternative Materials for the Blood Vessel Mimic Lab Electrospinning Process. 2017.

20. Tsui E. Effect of Media Composition on Confluent Endothelium Formation in Tissue Engineered Blood Vessels. 2018.

21. Frid MG, Kale VA, Stenmark KR. Mature vascular endothelium can give rise to smooth muscle cells via endothelial-mesenchymal transdifferentiation: In vitro analysis. Circ Res. 2002;90(11):1189-1196.

doi:10.1161/01.RES.0000021432.70309.28

22. Paszkowiak JJ, Dardik A, Haven N. Basic Science Review Arterial Wall Shear Stress: Observations from the Bench to the Bedside. Vol 37.; 2003.

23. Papaioannou T, Stefanadis C. Vascular Wall Shear Stress: Basic Principles and Methods. Hell J Cardiol. 2005;(46):9-15.

24. Stone PH, Coskun AU, Lucier M, et al. Intravascular hemodynamics and coronary artery disease: New insights and clinical implications. Hell J Cardiol. 2016;57(6):389-400. doi:10.1016/j.hjc.2016.11.019

25. Waller B, Orr C, Slack J, Pinkerton C. Anatomy, histology, and pathology of coronary arteries: A review relevant to new interventional and imaging techniques- 
part iv. Clin Cardiol. 1992;15:451-457. doi:10.1002/clc.4960150911

26. Soulis J V, Farmakis TM, Giannoglou GD. Wall Shear Stress in Normal Left Coronary Artery Tree. J Biomech. 2006;39(4):742-749.

27. Cassar A, Holmes DR, Rihal CS, Gersh BJ. Chronic coronary artery disease: Diagnosis and management. Mayo Clin Proc. 2009;84(12):1130-1146. doi:10.4065/mcp.2009.0391

28. Samijo S, Willigers J, Barkhuysen R, Kitslaar P. Wall shear stress in the human common carotid artery as function of age and gender. Cardiovasc Res. 1998;39(2):515-522. doi:10.1016/s0008-6363(98)00074-1

29. Limbu YR, Gurung G, Malla R, Rajbhandari R, Regmi SR. Assessment of carotid artery dimensions by ultrasound in non-smoker healthy adults of both sexes. Nepal Med Coll J. 2006;8(3):200-203. http://www.ncbi.nlm.nih.gov/pubmed/17203830.

30. Mayo Clinic. Carotid Angioplasty and Stenting. doi:10.1177/155989771101013

31. Saw SN, Dawn C, Biswas A, Mattar CNZ, Yap CH. Characterization of the in vivo wall shear stress environment of human fetus umbilical arteries and veins. Biomech Model Mechanobiol. 2017. doi:10.1007/s10237-016-0810-5

32. Lan Y, Yang Z, Huang M, Cui Z, Qi Y, Niu H. Morphological and structural changes of umbilical veins and clinical significance in preeclampsia. Hypertens Pregnancy. 2018;37(3):105-110. doi:10.1080/10641955.2017.1420799

33. Reneman RS, Hoeks APG. Wall shear stress as measured in vivo: Consequences for the design of the arterial system. Med Biol Eng Comput. 2008;46(5):499-507. doi:10.1007/s11517-008-0330-2

34. Chistiakov DA, Orekhov AN, Bobryshev Y V. Effects of shear stress on endothelial 
cells: go with the flow. Acta Physiol. 2017. doi:10.1111/apha.12725

35. Davies PF. Hemodynamic shear stress and the endothelium in cardiovascular pathophysiology. Nat Clin Pract Cardiovasc Med. 2009. doi:10.1038/ncpcardio1397

36. Givens C, Tzima E. Endothelial Mechanosignaling: Does One Sensor Fit All? Antioxid Redox Signal. 2016;25(7):373-388. doi:10.1089/ars.2015.6493

37. Dawson MC. Implementation of Physiologic Flow Conditions in a Blood Vessel Mimic Bioreactor System for the Evaluation of Intravascular Devices. 2009;(April).

38. Pérez L, Muñoz-Durango N, Riedel CA, et al. Endothelial-to-mesenchymal transition: Cytokine-mediated pathways that determine endothelial fibrosis under inflammatory conditions. Cytokine Growth Factor Rev. 2017;33:41-54. doi:10.1016/j.cytogfr.2016.09.002

39. Dejana E, Hirschi KK, Simons M. The molecular basis of endothelial cell plasticity. Nat Commun. 2017. doi:10.1038/ncomms14361

40. Cuttano R. KLF4 is a key determinant in the development and progression of Cerebral Cavernous Malformations. 2015.

41. Gasparics Á, Rosivall L, Krizbai IA, Sebe A. When the endothelium scores an own goal: endothelial cells actively augment metastatic extravasation through endothelial-mesenchymal transition. Am J Physiol - Hear Circ Physiol. 2016. doi:10.1152/ajpheart.00042.2016

42. Potenta S, Zeisberg E, Kalluri R. The role of endothelial-to-mesenchymal transition in cancer progression. Br J Cancer. 2008. doi:10.1038/sj.bjc.6604662 
43. Medici D, Kalluri R. Endothelial-mesenchymal transition and its contribution to the emergence of stem cell phenotype. Sminars Cancer Biol. 2015;25(8):713-724. doi:10.1097/MCA.0000000000000178.Endothelial

44. Zucker S. Cardiac Jelly and Its Roles in Heart Development. Sci J Lander Coll Arts Sci. 2011;4(4):23-29. https://touroscholar.touro.edu/cgi/viewcontent.cgi?referer=https://www.google.co. $\mathrm{uk} / \&$ httpsredir $=1 \&$ article $=1164 \&$ context $=$ sjlcas.

45. Monaghan MG, Van Handel B, Liebscher S, Schenke-Layland K, Layland SL, Linneweh M. Endocardial-to-mesenchymal transformation and mesenchymal cell colonization at the onset of human cardiac valve development. Development. 2016;143(3):473-482. doi:10.1242/dev.133843

46. Encyclopedia MM. Endocardial cushion defect. U.S. National Library of Medicine. https://medlineplus.gov/ency/article/007324.htm. Published 2019.

47. Lin F, Wang N, Zhang TC. The role of endothelial-mesenchymal transition in development and pathological process. IUBMB Life. 2012. doi:10.1002/iub.1059

48. Piera-Velazquez S, Mendoza F, Jimenez S. Endothelial to Mesenchymal Transition (EndoMT) in the Pathogenesis of Human Fibrotic Diseases. J Clin Med. 2016;5(4):45. doi:10.3390/jcm5040045

49. Evrard SM, Lecce L, Michelis KC, et al. Endothelial to mesenchymal transition is common in atherosclerotic lesions and is associated with plaque instability. Nat Commun. 2016;7(May). doi:10.1038/ncomms11853

50. Moonen JRAJ, Lee ES, Schmidt M, et al. Endothelial-to-mesenchymal transition contributes to fibro-proliferative vascular disease and is modulated by fluid shear 
stress. Cardiovasc Res. 2015;108(3):377-386. doi:10.1093/cvr/cvv175

51. Chiu J-J, Chien S. Effects of Distrubed Flow on Vascular Endothelium:

Pathophysiological Basis and Clinical Perspectives. Physiol rev. 2011;91(1):1-106. doi:10.1152/physrev.00047.2009.Effects

52. Tabas I, García-Cardeña G, Owens GK. Recent insights into the cellular biology of atherosclerosis. J Cell Biol. 2015. doi:10.1083/jcb.201412052

53. Medici D. Endothelial-Mesenchymal Transition in Regenerative Medicine. Stem Cells Int. 2016;2016. doi:10.1155/2016/6962801

54. Yang X, Liaw L, Prudovsky I, et al. Fibroblast Growth Factor Signaling in the Vasculature. Curr Atheroscler Rep. 2015. doi:10.1007/s11883-015-0509-6

55. Zeisberg EM, Tarnavski O, Zeisberg M, et al. Endothelial-to-mesenchymal transition contributes to cardiac fibrosis. Nat Med. 2007. doi:10.1038/nm1613

56. Susienka MJ, Medici D. Vascular endothelium as a novel source of stem cells for bioengineering. Biomatter. 2013. doi:10.4161/biom.24647

57. Matta C, Mobasheri A. Regulation of chondrogenesis by protein kinase C: Emerging new roles in calcium signalling. Cell Signal. 2014. doi:10.1016/j.cellsig.2014.01.011

58. Medici D, Shore EM, Lounev VY, Kaplan FS, Kalluri R, Olsen BR. Conversion of vascular endothelial cells into multipotent stem-like cells. Nat Med. 2010. doi:10.1038/nm.2252

59. Fu Y, Chang A, Chang L, Niessen K. Differential regulation of TGFbeta signaling pathways by notch in human endothelial cells. J Biol Chem. 2009;284(29):1945219462. doi:10.1074/jbc.m109.011833 
60. Lin F, Wang N, Zhang TC. The role of endothelial-mesenchymal transition in development and pathological process. IUBMB Life. 2012;64(9):717-723. doi:10.1002/iub.1059

61. Van Meeteren LA, Ten Dijke P. Regulation of endothelial cell plasticity by TGF- $\beta$. Cell Tissue Res. 2012;347(1):177-186. doi:10.1007/s00441-011-1222-6

62. Pardali E, Sanchez-Duffhues G, Gomez-Puerto MC, ten Dijke P. TGF- $\beta$-induced endothelial-mesenchymal transition in fibrotic diseases. Int J Mol Sci. 2017;18(10). doi:10.3390/ijms18102157

63. Li L, Chen L, Zang J, et al. C3a and C5a receptor antagonists ameliorate endothelialmyofibroblast transition via the $\mathrm{Wnt} / \beta$-catenin signaling pathway in diabetic kidney disease. Metabolism. 2015;64:597-610. doi:10.1016/j.metabol.2015.01.014

64. Mahler GJ, Farrar EJ, Butcher JT. Inflammatory cytokines promote mesenchymal transformation in embryonic and adult valve endothelial cells. Arterioscler Thromb Vasc Biol. 2013. doi:10.1161/ATVBAHA.112.300504

65. Maleszewska M, Moonen JRAJ, Huijkman N, van de Sluis B, Krenning G, Harmsen MC. IL-1 $\beta$ and TGF $\beta 2$ synergistically induce endothelial to mesenchymal transition in an NFkB-dependent manner. Immunobiology. 2013. doi:10.1016/j.imbio.2012.05.026

66. Maleszewska M, Gjaltema RAF, Krenning G, Harmsen MC. Enhancer of zeste homolog-2 (EZH2) methyltransferase regulates transgelin/smooth muscle-22 $\alpha$ expression in endothelial cells in response to interleukin-1 $\beta$ and transforming growth factor-\$2. Cell Signal. 2015. doi:10.1016/j.cellsig.2015.04.008

67. Guilpain P, Rojas M, Parolini O, et al. A Potential Link Between Oxidative Stress and 
Endothelial-to-Mesenchymal Transition in Systemic Sclerosis. Front Immunol | www.frontiersin.org. 2018;9. doi:10.3389/fimmu.2018.01985

68. Correia ACP, Moonen J-RAJ, Brinker MGL, Krenning G. FGF2 inhibits endothelialmesenchymal transition through microRNA-20a-mediated repression of canonical TGF- signaling. J Cell Sci. 2016;129(3):569-579. doi:10.1242/jcs.176248

69. Mahmoud MM, Serbanovic-Canic J, Feng S, et al. Shear stress induces endothelialTo-mesenchymal transition via the transcription factor Snail. Sci Rep. 2017;7(1):112. doi:10.1038/s41598-017-03532-Z

70. Krenning G, Barauna VG, Krieger JE, Harmsen MC, Moonen JRAJ. Endothelial Plasticity: Shifting Phenotypes through Force Feedback. Stem Cells Int. 2016;2016(January). doi:10.1155/2016/9762959

71. Boon RA, Fledderus JO, Volger OL, et al. KLF2 suppresses TGF- $\beta$ signaling in endothelium through induction of Smad7 and inhibition of AP-1. Arterioscler Thromb Vasc Biol. 2007;27(3):532-539. doi:10.1161/01.ATV.0000256466.65450.ce

72. Cowan C, Kohler E, Dugan T. Kruppel-Like Factor-4 Transcriptionally Regulates VE-Cadherin Expression and Endothelial Barrier Function. Circ Res. 2010. doi:10.1111/1467-8268.12301

73. Dong C, Zahir N. Biomechanics in Oncology.; 2018.

74. Azuma K, Ichimura K, Mita T, et al. Presence of $\alpha$-smooth muscle actin-positive endothelial cells in the luminal surface of adult aorta. Biochem Biophys Res Commun. 2009;380(3):620-626. doi:10.1016/j.bbrc.2009.01.135

75. Xiao L, Dudley AC. Fine-tuning Vascular Fate During Endothelial-Mesenchymal 
Transition. 2017;241(1):25-35. doi:10.1002/path.4814.Fine-tuning

76. Schwartz M, Conway D. Lessons from the endothelial junctional mechanosensory complex. F1000 Biol Rep. 2012;4(January):2-7. doi:10.3410/B4-1

77. Chen L-J, Wang W-L, Chiu J-J. Vascular Endothelial Mechanosensors in Response to Fluid Shar Stress.; 2016.

78. Moriguchi T, Sumpio BE. PECAM-1 phosphorylation and tissue factor expression in HUVECs exposed to uniform and disturbed pulsatile flow and chemical stimuli. $J$ Vasc Surg. 2015;61(2):481-488. doi:10.1016/j.jvs.2013.09.059

79. Osawa M, Masuda M, Kusano KI, Fujiwara K. Evidence for a role of platelet endothelial cell adhesion molecule-1 in endothelial cell mechanosignal transduction: Is it a mechanoresponsive molecule? J Cell Biol. 2002;158(4):773785. doi: $10.1083 /$ jcb. 200205049

80. Conway DE, Schwartz MA. Mechanotransduction of shear stress occurs through changes in ve-cadherin and pecam-1 tension: Implications for cell migration. Cell Adhes Migr. 2015;9(5):335-339. doi:10.4161/19336918.2014.968498

81. Conway DE, Breckenridge MT, Hinde E, Gratton E, Chen CS, Schwartz MA. Fluid shear stress on endothelial cells modulates mechanical tension across VE-cadherin and PECAM-1. Curr Biol. 2013;23(11):1024-1030. doi:10.1016/j.cub.2013.04.049

82. Privratsky J, Newman D, Newman P. PECAM-1: Conflicts of Interest in Inflammation. Life Sci. 2010. doi:10.1016/j.den.2011.01.002.The

83. Harry BL, Sanders JM, Feaver RE, et al. Endothelial Cell PECAM-1 Promotes Atherosclerotic Lesions in Areas of Disturbed Flow in ApoE-Deficient Mice. Arterioscler Thromb Vasc Biol. 2008. 
doi:10.1161/ATVBAHA.108.164707.Endothelial

84. Short B. How VE-cadherin goes with the flow. J Cell Biol. 2015;208(7):861. doi:10.1083/jcb.2087if

85. Lampugnani MG, Zanetti A, Corada M, et al. Contact inhibition of VEGF-induced proliferation requires vascular endothelial cadherin, ??-catenin, and the phosphatase DEP-1/CD148. J Cell Biol. 2003;161(4):793-804. doi:10.1083/jcb.200209019

86. Vestweber D. VE-cadherin: The major endothelial adhesion molecule controlling cellular junctions and blood vessel formation. Arterioscler Thromb Vasc Biol. 2008;28(2):223-232. doi:10.1161/ATVBAHA.107.158014

87. Wright TJ, Leach L, Shaw PE, Jones P. Dynamics of vascular endothelial-cadherin and $\beta$-catenin localization by vascular endothelial growth factor-induced angiogenesis in human umbilical vein cells. Exp Cell Res. 2002;280(2):159-168. doi:10.1006/excr.2002.5636

88. Coon BG, Baeyens N, Han J, et al. Intramembrane binding of VE-cadherin to VEGFR2 and VEGFR3 assembles the endothelial mechanosensory complex. $J$ Cell Biol. 2015;208(7):975-986. doi:10.1083/jcb.201408103

89. Del Rosso M. uPAR in angiogenesis regulation. Blood. 2011;117(15):3941-3943. doi:10.1182/blood-2011-02-337733

90. Brunner PM, Heier PC, Mihaly-Bison J, Priglinger U, Binder BR, Prager GW. Density enhanced phosphatase-1 down-regulates urokinase receptor surface expression in confluent endothelial cells. Blood. 2011;117(15):4154-4161. doi:10.1182/blood-2010-09-307694 
91. Conway DE, Coon BG, Budatha M, et al. VE-Cadherin Phosphorylation Regulates Endothelial Fluid Shear Stress Responses through the Polarity Protein LGN. Curr Biol. 2017;27(14):2219-2225.e5. doi:10.1016/j.cub.2017.06.020

92. dela Paz NG, Walshe TE, Leach LL, Saint-Geniez M, D’Amore PA. Role of shearstress-induced VEGF expression in endothelial cell survival. J Cell Sci. 2012;125(4):831-843. doi:10.1242/jcs.084301

93. Ukropec JA, Hollinger MK, Woolkalis MJ. Regulation of VE-cadherin linkage to the cytoskeleton in endothelial cells exposed to fluid shear stress. Exp Cell Res. 2002;273(2):240-247. doi:10.1006/excr.2001.5453

94. Urbich C, Stein M, Reisinger K, et al. Fluid shear stress-induced transcriptional activation of the vascular endothelial growth factor receptor-2 gene requires Sp1dependent DNA binding. FEBS Lett. 2003;535(1-3):87-93. doi:10.1016/S00145793(02)03879-6

95. Kadohama T, Nishimura K, Hoshino Y. Effects of Different Types of Fluid Shear Stress on Endothelial Cell Proliferation and Survival. J Cell Physiol. 2007. doi:10.1002/JCP

96. Harris LJ, Abdollahi H, Zhang P, McIlhenny S, Tulenko TN, DiMuzio PJ. Differentiation of adult stem cells into smooth muscle for vascular tissue engineering. J Surg Res. 2011;168(2):306-314. doi:10.1016/j.jss.2009.08.001

97. Medici D, Shore EM, Lounev VY, Kaplan FS. Conversion of Vascular Endothelial Cells into Multipotent Stem-like cells. Nat Med. 2009;6(12):247-253. doi:10.1111/j.1743-6109.2008.01122.x.Endothelial

98. Zhang Y, Wu X, Li Y, et al. Endothelial to mesenchymal transition contributes to 
arsenic-trioxide-induced cardiac fibrosis. Sci Rep. 2016;6(September):1-12. doi:10.1038/srep33787

99. Dunleavey JM, Kim DJ, Monaghan-Benson E, et al. Tumor Endothelial Cells with Distinct Patterns of TGF $\beta$-Driven Endothelial-to-Mesenchymal Transition. Cancer Res. 2015;75(7):1244-1254. doi:10.1158/0008-5472.can-14-1616

100. ThermoFisher Scientific. Basic Principles of RT-qPCR. https://www.thermofisher.com/us/en/home/brands/thermo-scientific/molecularbiology/molecular-biology-learning-center/molecular-biology-resourcelibrary/spotlight-articles/basic-principles-rt-qpcr.html.

101. Real-time PCR Handbook. Life Technologies. doi:10.1006/excr.2001.5278

102. Bio Rad. Introduction to PCR Primer \& Probe Chemistries. http://www.biorad.com/en-us/applications-technologies/introduction-pcr-primer-probechemistries?ID=LUSOJW3Q3.

103. Smith CJ, Osborn AM. Advantages and limitations of quantitative PCR (Q-PCR)based approaches in microbial ecology. FEMS Microbiol Ecol. 2009;67(1):6-20. doi:10.1111/j.1574-6941.2008.00629.x

104. Nick D. Interpreting Melt Curves : An Indicator, Not a Diagnosis. Integr DNA Technol Inc. 2014:1-7. doi:10.5209/rev_HICS.2014.v19.45062

105. Prada-Arismendy J, Castellanos JE. Real time PCR. Application in dengue studies. Colomb Med. 2011;42(June):243-258. http://www.bioline.org.br/pdf?rc11033.

106. Ghosh AK, Nagpal V, Covington JW, Michaels M a., Vaughan DE. Molecular basis of cardiac endothelial-to-mesenchymal transition (EndMT): Differential expression of microRNAs during EndMT. Cell Signal. 2012;29(6):997-1003. 
doi:10.1016/j.biotechadv.2011.08.021.Secreted

107. Liu Y, Yuan X, Li W, Cao Q, Shu Y. Aspirin-triggered resolvin D1 inhibits TGF-1induced EMT through the inhibition of the mTOR pathway by reducing the expression of PKM2 and is closely linked to oxidative stress. Int J Mol Med. 2016;38(4):1235-1242. doi:10.3892/ijmm.2016.2721

108. Cao Y, Feng B, Chen S, Chu Y, Chakrabarti S. Mechanisms of endothelial to mesenchymal transition in the retina in diabetes. Investig Ophthalmol Vis Sci. 2014;55(11):7321-7331. doi:10.1167/iovs.14-15167

109. Cooley B, Nevado J, Mellad J, Yang D. TGF-Signaling Mediates Endothelial-toMesenchymal Transition (EndMT) During Vein Graft Remodeling. Sci Transl Med. 2014;6(227):227ra34-227ra34. doi:10.1126/scitranslmed.3006927

110. National Institutes of Health. GUSB gene. U.S. National Library of Medicine. https://ghr.nlm.nih.gov/gene/GUSB. Published 2019.

111. Żyżyńska-Granica B, Koziak K. Identification of Suitable Reference Genes for Real-Time PCR Analysis of Statin-Treated Human Umbilical Vein Endothelial Cells. PLoS One. 2012;7(12):e51547. doi:10.1371/journal.pone.0051547

112. Li T, Diao H, Zhao L, et al. Identification of suitable reference genes for real-time quantitative PCR analysis of hydrogen peroxide-treated human umbilical vein endothelial cells. BMC Mol Biol. 2017;18(1):1-8. doi:10.1186/s12867-017-0086-z

113. ThermoFisher Scientific. Poor Efficiency of PCR. https://www.thermofisher.com/us/en/home/life-science/pcr/real-time-pcr/real-timepcr-learning-center/real-time-pcr-basics/real-time-pcr-troubleshooting-tool/geneexpression-quantitation-troubleshooting/poor-pcr-efficiency.html. 
114. QIAGEN. RNeasy mini handbook. Sample Assay Technol. 2012;(June):1-80. doi:10.1007/978-0-387-77674-3

115. Thermo-Scientific. NanoDrop 2000 / 2000c Spectrophotometer. 2000.

116. Biorad. iScript cDNA Synthesis Kit. http://www.biorad.com/webroot/web/pdf/lsr/literature/4106228.pdf.

117. Applied Biosystems. Fast SYBR Green Master Mix Protocol.

118. Integrated DNA Technologies. qPCR Terminology — What Does It Mean? https://www.idtdna.com/pages/education/decoded/article/qpcr-terminology-whatdoes-it-mean-. Published 2013.

119. Lutz KA, Hart ML, Aicher WK, et al. Smooth Muscle-Like Cells Generated from Human Mesenchymal Stromal Cells Display Marker Gene Expression and Electrophysiological Competence Comparable to Bladder Smooth Muscle Cells. PLoS One. 2015;10(12):e0145153. doi:10.1371/journal.pone.0145153

120. Balaguru UM, Sundaresan L, Manivannan J, et al. Disturbed flow mediated modulation of shear forces on endothelial plane: A proposed model for studying endothelium around atherosclerotic plaques. Sci Rep. 2016;6(May):1-15. doi:10.1038/srep27304

121. Matlock B. Assessment of Nucleic Acid Purity - NanoDrop Spectrophotometers. ThermoFisher Sci. 2015:1-3. www.thermoscientific.com.

122. Cai D-X, Quan Y, He P-J, Tan H-B, Xu Y-Q. Dynamic Perfusion Culture of Human Outgrowth Endothelial Progenitor Cells on Demineralized Bone Matrix In Vitro. Med Sci Monit. 2016;22:4037-4045. doi:10.12659/msm.897884

123. Khan O, Sefton M. Perfusion and Characterization of an Endothelial Cell-Seeded 
Modular Tissue Engineered Construct Formed in a Microfluidic Remodeling Chamber. Biomaterials. 2010;31(32). doi:10.1158/0008-5472.CAN-104002.BONE

124. Miao H, Hu YL, Shiu YT, et al. Effects of flow patterns on the localization and expression of VE-cadherin at vascular endothelial cell junctions: In vivo and in vitro investigations. J Vasc Res. 2005;42(1):77-89. doi:10.1159/000083094

125. Agarwal S, Wendorff JH, Greiner A. Use of electrospinning technique for biomedical applications. Polymer (Guildf). 2008;49(26):5603-5621. doi:10.1016/j.polymer.2008.09.014

126. Hodgkinson T, Yuan X-F, Bayat A. Electrospun silk fibroin fiber diameter influences in vitro dermal fibroblast behavior and promotes healing of ex vivo wound models. J Tissue Eng. 2014;5:204173141455166.

doi:10.1177/2041731414551661

127. Chen M, Patra PK, Warner SB, Bhowmick S. Role of Fiber Diameter in Adhesion and Proliferation of NIH 3T3 Fibroblast on Electrospun Polycaprolactone Scaffolds. Tissue Eng. 2007;13(3):579-587. doi:10.1089/ten.2006.0205

128. Wee-Eong. Influence of fiber diameter on cells. ElectrospinTech. 2015. http://electrospintech.com/cellfiberdiameter.html\#.WtcnaWaZOgR.

129. Whited B, Rylander MN. The Influence of Electrospun Scaffold Topography ln Endothelial Cell Morphology, Alignment, and Adhesion in Response to Fluid Flow. Biotechnol Bioeng. 2014;111(1). doi:10.1002/bit.24995

130. Murphy CM, O’Brien FJ. Understanding the effect of mean pore size on cell activity in collagen-glycosaminoglycan scaffolds. Cell Adhes Migr. 2010;4(3):377-381. 
doi:10.1002/chem.201700140

131. Murphy CM, Haugh MG, O 'brien FJ, Murphy CM, Haugh MG, O 'brien FJ. The effect of mean pore size on cell attachment, proliferation and migration in collagen glycosaminoglycan scaffolds for tissue engineering. Citation Attribution-NonCommercial-ShareAlike 1.0 Investigating the effect of mean pore size on cell attachment, . Biomaterials. 2010;31(3):461-466.

132. Chang H-I, Wang Y. Cell Responses to Surface and Architecture of Tissue Engineering Scaffolds. Regen Med Tissue Eng - Cells Biomater. 2011. doi:10.5772/21983

133. Saunders K, D’Amore P. An in vitro Model for Cell-Cell Interactions. Vitr Cell Dev Biol. 1992;28(7):521-528.

134. Bružauskaitė I, Bironaitè D, Bagdonas E, Bernotienè E. Scaffolds and cells for tissue regeneration: different scaffold pore sizes—different cell effects. Cytotechnology. 2016;68(3):355-369. doi:10.1007/s10616-015-9895-4

135. Wang X, Lou T, Zhao W, Song G, Li C, Cui G. The effect of fiber size and pore size on cell proliferation and infiltration in PLLA scaffolds on bone tissue engineering. J Biomater Appl. 2015;30(10):1545-1551. doi:10.1177/0885328216636320

136. Ahmed M, Ramos T, Wieringa P, Blitterswijk C Van, Boer J De, Moroni L. Geometric constraints of endothelial cell migration on electrospun fibres. Sci Rep. 2018;8(1):1-10. doi:10.1038/s41598-018-24667-7

137. Jalali S, Tafazzoli-Shadpour M, Haghighipour N, Omidvar R, Safshekan F. Regulation of Endothelial Cell Adherence and Elastic Modulus by Substrate Stiffness. Cell Commun Adhes. 2015;22(2-6):79-89. 
doi:10.1080/15419061.2016.1265949

138. Engler AJ, Sen S, Sweeney HL, Discher DE. Matrix Elasticity Directs Stem Cell Lineage Specification. Cell. 2006. doi:10.1016/j.cell.2006.06.044

139. Heath DE, Lannutti JJ, Cooper SL. Electrospun scaffold topography affects endothelial cell proliferation, metabolic activity, and morphology. J Biomed Mater Res - Part A. 2010;94(4):1195-1204. doi:10.1002/jbm.a.32802

140. Galie PA, Van Oosten A, Chen CS, Janmey PA. Application of multiple levels of fluid shear stress to endothelial cells plated on polyacrylamide gels. Lab Chip. 2015. doi:10.1039/c4lc01236d

141. Sung HJ, Meredith C, Johnson C, Galis ZS. The effect of scaffold degradation rate on three-dimensional cell growth and angiogenesis. Biomaterials. 2004;25(26):5735-5742. doi:10.1016/j.biomaterials.2004.01.066

142. Renehan A, Booth C, Potten C. What is apoptosis, and why is it important? Br Med J. 2001. doi:10.1002/9781118951149.ch1

143. Elmore S. Apoptosis: A Review of Programmed Cell Death. Toxicol Pathol. 2007;35(4):495-516. doi:10.1080/01926230701320337

144. Gregory CD, Pound JD. Microenvironmental influences of apoptosis in vivo and in vitro. Apoptosis. 2010;15(9):1029-1049. doi:10.1007/s10495-010-0485-9

145. Jin Z, El-Deiry WS. Overview of cell death signaling pathways. Cancer Biol Ther. 2005;4(2):139-163. doi:10.4161/cbt.4.2.1508

146. ImmunoChemistry Technologies. Apoptosis: Function and Pathways. https://immunochemistry.com/educational-material/apoptosis-function-pathways/.

147. Kumar S. Measurement of caspase activity in cells undergoing apoptosis. Apoptosis 
Methods Protoc. 2004;228:19-30. doi:10.1385/1-59259-812-9:019

148. Green DR. Apoptotic Pathways : The Roads to Ruin. 1998;94:695-698.

149. Bio Cat. Apoptosis Detection (Phosphatidylserin/Annexin based).

https://www.biocat.com/cell-biology/apoptosis/apoptosis-detectionphosphatidylserin-annexin-based.

150. Promo Cell. Mitochondrial Apoptosis Staining Kit. https://www.promocell.com/product/mitochondrial-apoptosis-staining-kit/.

151. Watanabe M, Hitomi M, Van der Wee K, et al. The pros and cons of apoptosis assays for use in the study of cells, tissues, and organs. Microsc Microanal. 2002;8(5):375-391. doi:10.1017/S1431927602010346

152. ThermoFisher Scientific. Image-iT ${ }^{\mathrm{TM}}$ LIVE Green Caspase Detection Kits. https://www.thermofisher.com/document-connect/documentconnect.html?url=https://assets.thermofisher.com/TFSAssets/LSG/manuals/mp35104.pdf\&title=SW1hZ2UtaVQgTElWRSBHcmVlbiB DYXNwYXNIIERldGVjdGlvbiBLaXRz. Published 2005.

153. Bačáková L, Filová E, Rypáček F, Švorčík V, Starý V. Cell Adhesion on Artificial Materials for Tissue Engineering. Physiol Res. 2004;53(SUPPL. 1). doi:10.1088/0957-4484/21/48/485703

154. Khalili AA, Ahmad MR. A Review of cell adhesion studies for biomedical and biological applications. Int J Mol Sci. 2015;16(8):18149-18184. doi:10.3390/ijms 160818149

155. Wozniak MA, Modzelewska K, Kwong L, Keely PJ. Focal adhesion regulation of cell behavior. 2004;1692:103-119. doi:10.1016/j.bbamcr.2004.04.007 
156. Huveneers S, Danen EHJ. Adhesion signaling - crosstalk between integrins, Src and Rho. 2009;2. doi:10.1242/jcs.039446

157. Pasqualini FS, Agarwal A, O’Connor BB, Liu Q, Sheehy SP, Parker KK. Traction force microscopy of engineered cardiac tissues. PLoS One. 2018;13(3):1-14. doi:10.1371/journal.pone.0194706

158. Ju L, Chen Y, Li K, et al. Dual Biomembrane Force Probe enables single-cell mechanical analysis of signal crosstalk between multiple molecular species. Sci Rep. 2017;7(1):1-12. doi:10.1038/s41598-017-13793-3

159. Röcker T, Greiner A. Electrospinning of poly-L-lactide nanofibers on liquid reservoir collectors. E-Polymers. 2008;(March). doi:Artn 111

160. You Y, Lee SJ, Min BM, Park WH. Effect of solution properties on nanofibrous structure of electrospun poly(lactic-co-glycolic acid). J Appl Polym Sci. 2006;99(3):1214-1221. doi:10.1002/app.22602

161. Park JA, Kim SB. Preparation and characterization of antimicrobial electrospun poly(vinyl alcohol) nanofibers containing benzyl triethylammonium chloride. React Funct Polym. 2015;93(1):30-37. doi:10.1016/j.reactfunctpolym.2015.05.008

162. Na H, Liu X, Li J, Zhao Y, Zhao C, Yuan X. Formation of core/shell ultrafine fibers of PVDF/PC by electrospinning via introduction of PMMA or BTEAC. Polymer (Guildf). 2009;50(26):6340-6349. doi:10.1016/j.polymer.2009.10.061

163. Tong H, Wang M. Electrospinning of Fibrous PHBV Tissue Engineering Scaffolds: Fiber Diameter Control, Fiber Alignment and Mechanical Properties. Inst Electr Electron Eng. 2008:535-538.

164. Park JA, Kim SB. Antimicrobial filtration with electrospun poly(vinyl alcohol) 
nanofibers containing benzyl triethylammonium chloride: Immersion, leaching, toxicity, and filtration tests. Chemosphere. 2017;167:469-477.

doi:10.1016/j.chemosphere.2016.10.030

165. Kwak GH, Choi SH, Kim HY. Dimethyl sulfoxide elevates hydrogen peroxidemediated cell death in Saccharomyces cerevisiae by inhibiting the antioxidant function of methionine sulfoxide reductase A. BMB Rep. 2010;43(9):622-628. doi:10.3858/BMBRep.2010.43.9.622

166. ISO 10993-5.

167. Ko YG, Park JH, Lee JB, et al. Growth behavior of endothelial cells according to electrospun poly(D,L-lactic-co-glycolic acid) fiber diameter as a tissue engineering scaffold. Tissue Eng Regen Med. 2016;13(4):343-351. doi:10.1007/s13770-0160053-7

168. Azimi B, Nourpanah P, Rabiee M, Arbab S. Poly (lactide-co-glycolide) Fiber: An Overview. J Eng Fiber Fabr. 2014;9(1).

169. Halaidych O V., Freund C, van den Hil F, et al. Inflammatory Responses and Barrier Function of Endothelial Cells Derived from Human Induced Pluripotent Stem Cells. Stem Cell Reports. 2018;10(5):1642-1656.

doi:10.1016/j.stemcr.2018.03.012

170. Stervbo U. The effects of resveratrol on cell cycle, apoptosis, and NF-kB activation. 2005.

171. Darzynkiewicz Z, Pozarowski P. Fluorochrome-Labeled Inhibitors of Caspases: Convenient In Vitro and In Vivo Markers of Apoptotic Cells for Cytometric Analysis. 2011;682:103-114. doi:10.1007/978-1-60327-409-8 
172. Chevallet M, Diemer H, Dorssealer A Van, Villiers C, Rabilloud T. Toward a better analysis of secreted proteins : the example of the myeloid cells secretome. Proteomics. 2007;7(11):1757-1770. doi:10.1002/pmic.200601024

173. Douglas G, Van Kampen E, Hale AB, et al. Endothelial cell repopulation after stenting determines in-stent neointima formation: Effects of bare-metal vs. drugeluting stents and genetic endothelial cell modification. Eur Heart $J$. 2013;34(43):3378-3388. doi:10.1093/eurheartj/ehs240

174. Lucas AD, Merritt K, Hitchins VM, et al. Residual Ethylene Oxide in Medical Devices and Device Material. J Biomed Mater Res - Part B Appl Biomater. 2003;66(2):548-552. doi:10.1002/jbm.b.10036

175. Holy CE, Cheng C, Davies JE, Shoichet MS. Optimizing the sterilization of PLGA scaffolds for use in tissue engineering. Biomaterials. 2001;22(1):25-31. doi:10.1016/S0142-9612(00)00136-8 


\section{APPENDICES}

Appendix A: Preverified Primer Sequences and Characteristics

\begin{tabular}{|c|c|c|c|c|c|}
\hline $\begin{array}{l}\text { Primer } \\
\text { Name }\end{array}$ & Primer Sequence & $\begin{array}{l}\text { Exon } \\
\text { Locati } \\
\text { on }\end{array}$ & $\begin{array}{l}\text { Molecul } \\
\text { ar } \\
\text { Weight } \\
\text { (Da) }\end{array}$ & $\begin{array}{l}\text { Extinction } \\
\text { Coefficient } \\
\left(\mathrm{L} /\left(\mathrm{mol}^{*} \mathrm{c}\right.\right. \\
\mathrm{m}))\end{array}$ & $\begin{array}{l}\mathrm{GC} \\
\text { Conte } \\
\text { nt }(\%)\end{array}$ \\
\hline $\begin{array}{l}\text { PECAM } \\
1 \text { Primer } \\
1\end{array}$ & $\begin{array}{l}5^{\prime}- \\
\text { ATTGCTCTGGTCACTTCTCC- } \\
3 \text { ' }\end{array}$ & $1 b-2 b$ & $6,009.9$ & $172,300.0$ & 50.0 \\
\hline $\begin{array}{l}\text { PECAM } \\
1 \text { Primer } \\
2\end{array}$ & 5'-CAGGCCCCATTGTTCCC-3' & $1 b-2 b$ & $5,082.3$ & 146,700 & 64.7 \\
\hline $\begin{array}{l}\text { CDH5 } \\
\text { (VE- } \\
\text { cadherin } \\
\text { ) Primer } \\
1\end{array}$ & $\begin{array}{l}\text { 5'- } \\
\text { TGCCCACATATTCTCCTTTG } \\
\text { AG-3' }\end{array}$ & $2-3$ & $6,636.4$ & $198,100.0$ & 45.5 \\
\hline $\begin{array}{l}\text { CDH5 } \\
\text { (VE- } \\
\text { cadherin } \\
\text { ) Primer } \\
1\end{array}$ & $\begin{array}{l}\text { 5'- } \\
\text { GAACCAGATGCACATTGATG } \\
\text { AAG-3' }\end{array}$ & $2-3$ & $7,105.7$ & $237,000.0$ & 43.5 \\
\hline $\begin{array}{l}\text { KDR } \\
\text { (VEGFR } \\
\text { 2) } \\
\text { Primer 1 }\end{array}$ & $\begin{array}{l}\text { 5'- } \\
\text { GAGGATCTTGAGTTCAGACA } \\
\text { TGAG-3' }\end{array}$ & $18-19$ & $7,456.9$ & $244,800.0$ & 45.8 \\
\hline
\end{tabular}




\begin{tabular}{|c|c|c|c|c|c|}
\hline $\begin{array}{l}\text { KDR } \\
\text { (VEGFR } \\
\text { 2) } \\
\text { Primer } 2\end{array}$ & $\begin{array}{l}5^{\prime}- \\
\text { TTGGAATTGACAAGACAGCA } \\
\text { AC-3' }\end{array}$ & $18-19$ & $6,776.5$ & $224,000.0$ & 40.9 \\
\hline $\begin{array}{l}\text { ACTA2 } \\
(\alpha \text { SMA }) \\
\text { Primer } 1\end{array}$ & $\begin{array}{l}\text { 5'- } \\
\text { CTGTTGTAGGTGGTTTCATG } \\
\text { GA-3' }\end{array}$ & $8-9$ & $6,827.5$ & $211,700.0$ & 45.5 \\
\hline $\begin{array}{l}\text { ACTA2 } \\
(\alpha \text { SMA }) \\
\text { Primer } 2\end{array}$ & $\begin{array}{l}\text { 5'- } \\
\text { AGAGTTACGAGTTGCCTGAT } \\
\text { G-3' }\end{array}$ & $8-9$ & $6,501.3$ & $209,000.0$ & 47.6 \\
\hline $\begin{array}{l}\text { CNN1 } \\
\text { Primer 1 }\end{array}$ & $\begin{array}{l}5^{\prime}- \\
\text { CATGAAGTTGTTGCCGATGC } \\
-3,\end{array}$ & $1-2$ & $6,148.0$ & $189,400.0$ & 50.0 \\
\hline $\begin{array}{l}\text { CNN1 } \\
\text { Primer } 2\end{array}$ & $\begin{array}{l}\text { 5'- } \\
\text { TCAGCCGAGGTTAAGAACA } \\
\text { AG-3' }\end{array}$ & $1-2$ & $6,488.3$ & $216,700.0$ & 47.6 \\
\hline $\begin{array}{l}\text { ACTB } \\
\text { Primer } 1\end{array}$ & $\begin{array}{l}\text { 5'-CCTTGCACATGCCGGAG- } \\
\text { 3', }\end{array}$ & $1-2$ & 5,171.4 & $155,300.0$ & 64.7 \\
\hline $\begin{array}{l}\text { ACTB } \\
\text { Primer } 2\end{array}$ & 5'-ACAGAGCCTCGCCTTTG-3' & $1-2$ & $5,146.4$ & $154,700.0$ & 58.8 \\
\hline $\begin{array}{l}\text { GUS } \beta \\
\text { Primer } 1\end{array}$ & $\begin{array}{l}5^{\prime}- \\
\text { GTTTTTGATCCAGACCCAGA } \\
\text { TG-3' }\end{array}$ & $10-11$ & $6,725.4$ & $210,600.0$ & 45.5 \\
\hline $\begin{array}{l}\text { GUS } \beta \\
\text { Primer } 2\end{array}$ & $\begin{array}{l}5^{\prime}- \\
\text { GCCCATTATTCAGAGCGAGT } \\
\text { A-3' }\end{array}$ & $10-11$ & $6,430.2$ & $208,900.0$ & 47.6 \\
\hline
\end{tabular}


Appendix B: Primer Efficiency Testing SOP

Appendix C: RNA Isolation and Evaluation SOP

Appendix D: cDNA through Reverse Transcription SOP

Appendix E: qPCR SOP

Appendix F: qPCR Data Analysis SOP 
Appendix G: Primer Efficiency Plots

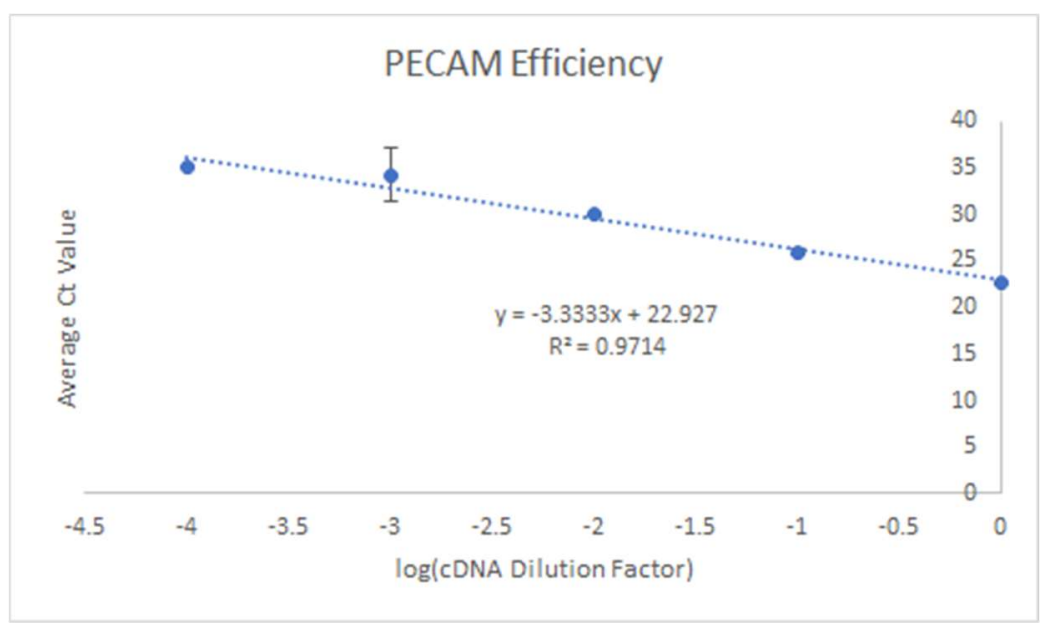



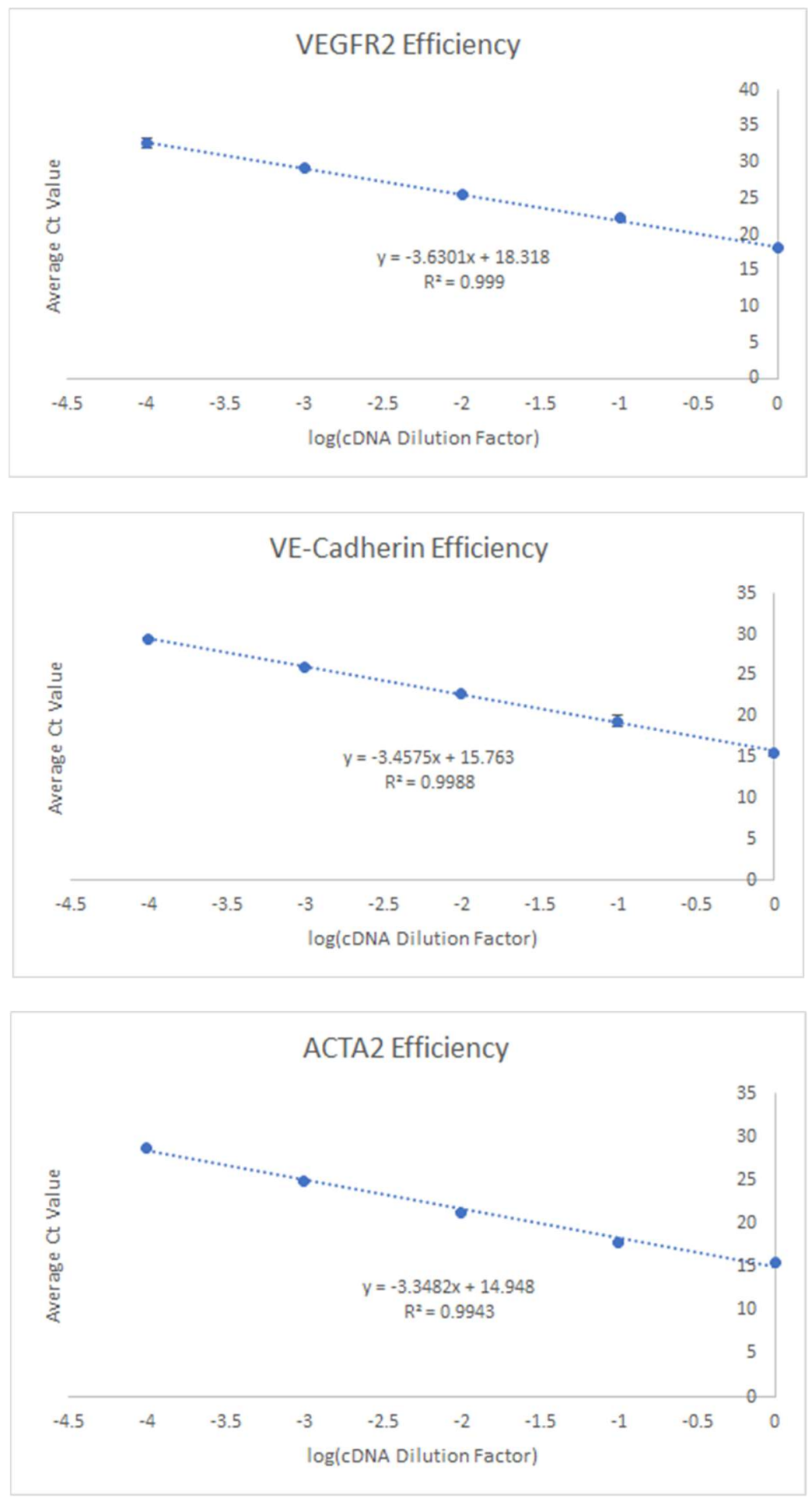

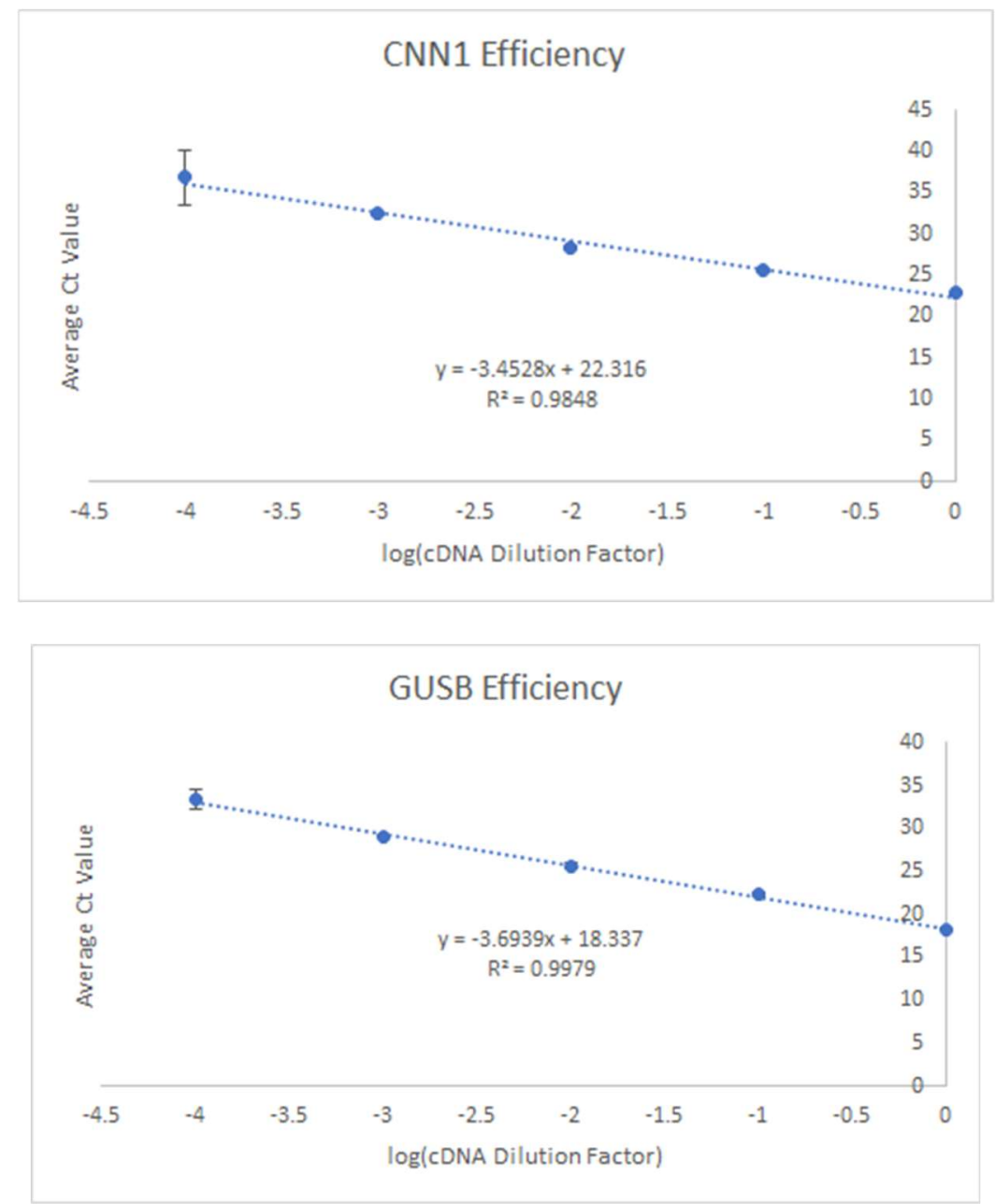

\section{Appendix H: BVM Conditioning SOP}

Appendix I: Endothelial Cell-only BVM Sodding SOP

Appendix J: Apoptosis Kit Staining SOP

Appendix K: Cytotoxicity Elution Assay SOP 
Appendix L: Scaffold Adhesion Assay SOP

Appendix M: Positive Control Test Representative Images

\begin{tabular}{|l|l|l|l|}
\hline & Hoechst & Caspase & Propidium Iodide \\
\hline
\end{tabular}




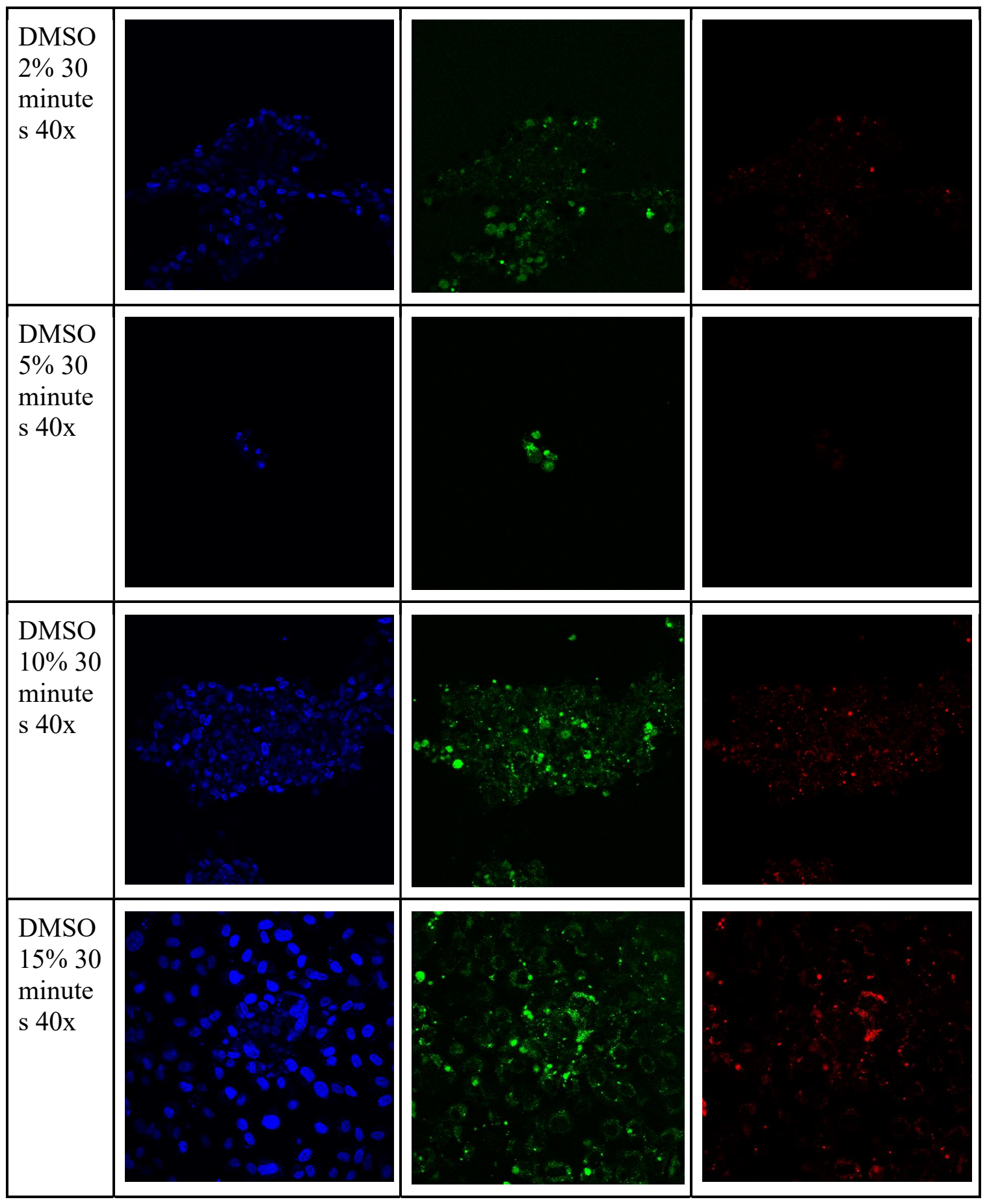




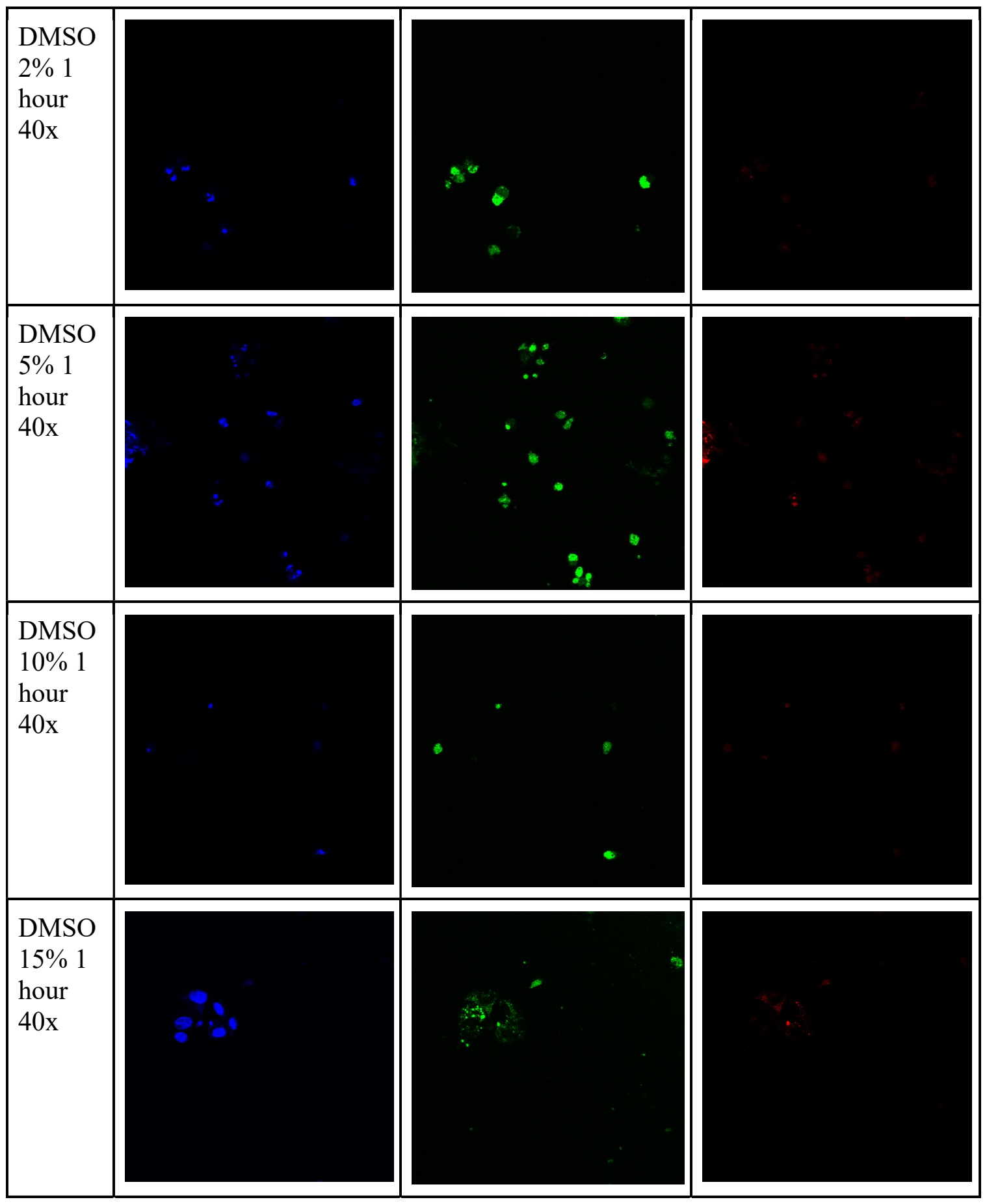




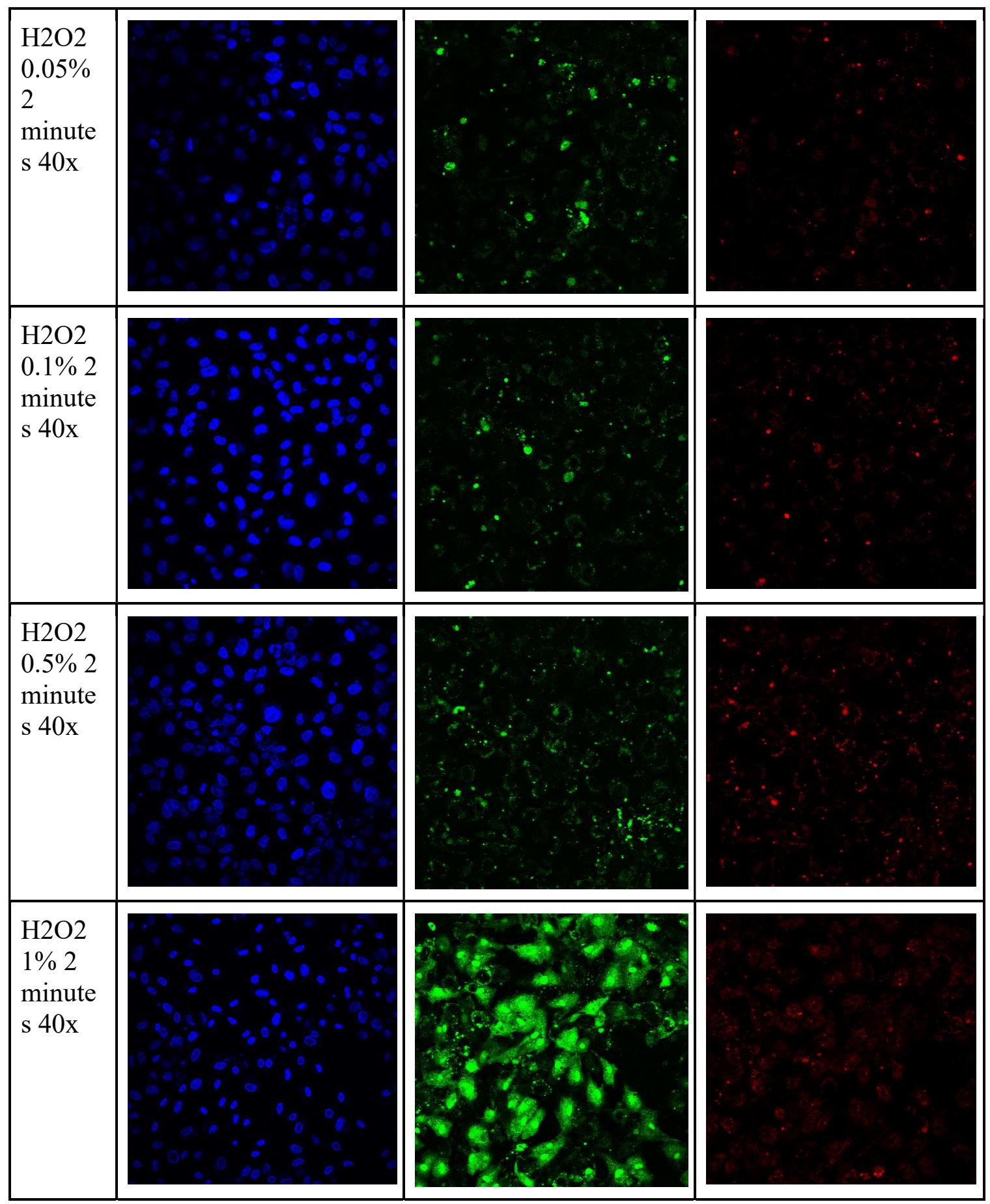




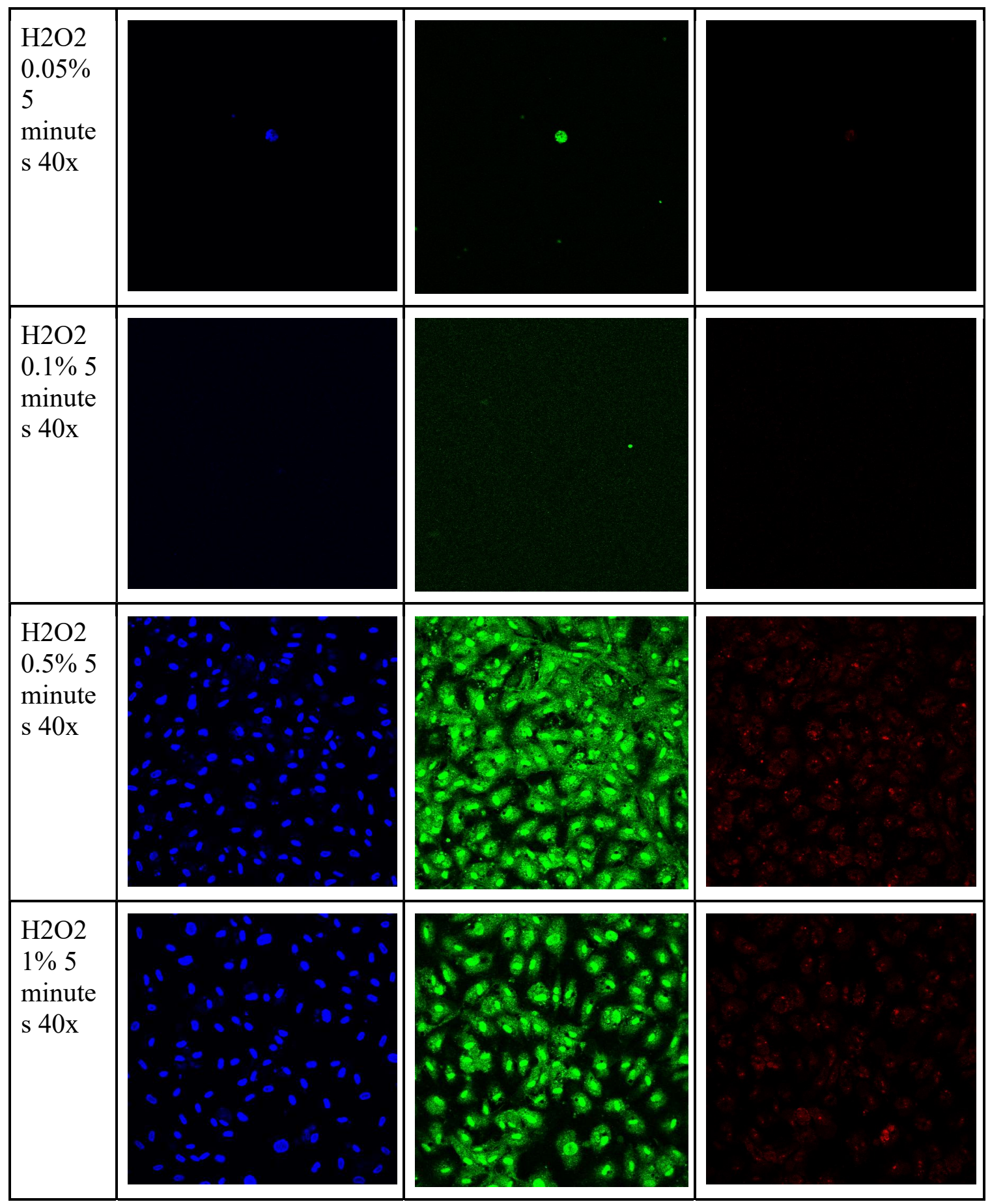


Appendix N: Cytotoxicity Elution Assay Representative Images

24 Hours, 40x

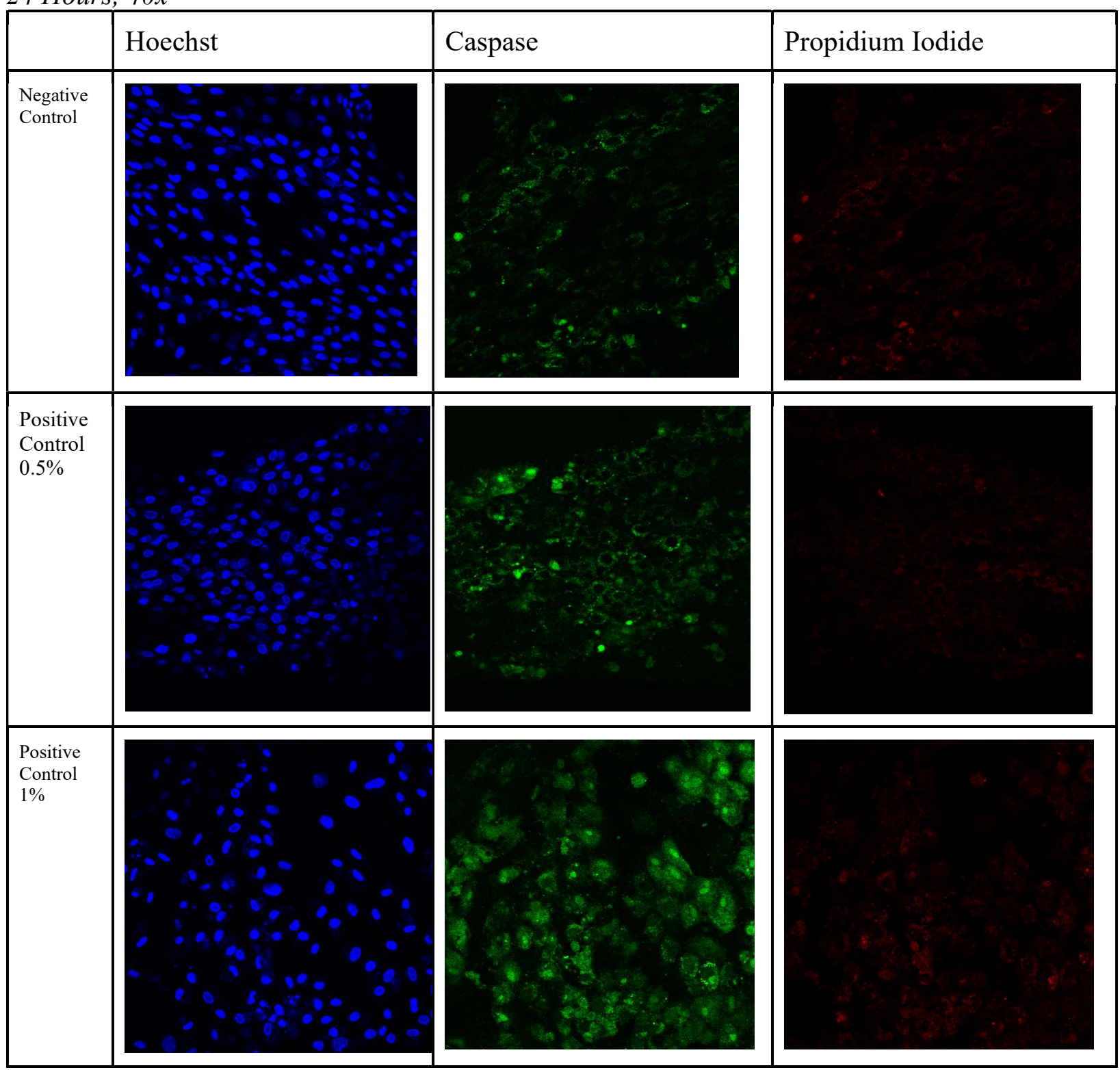




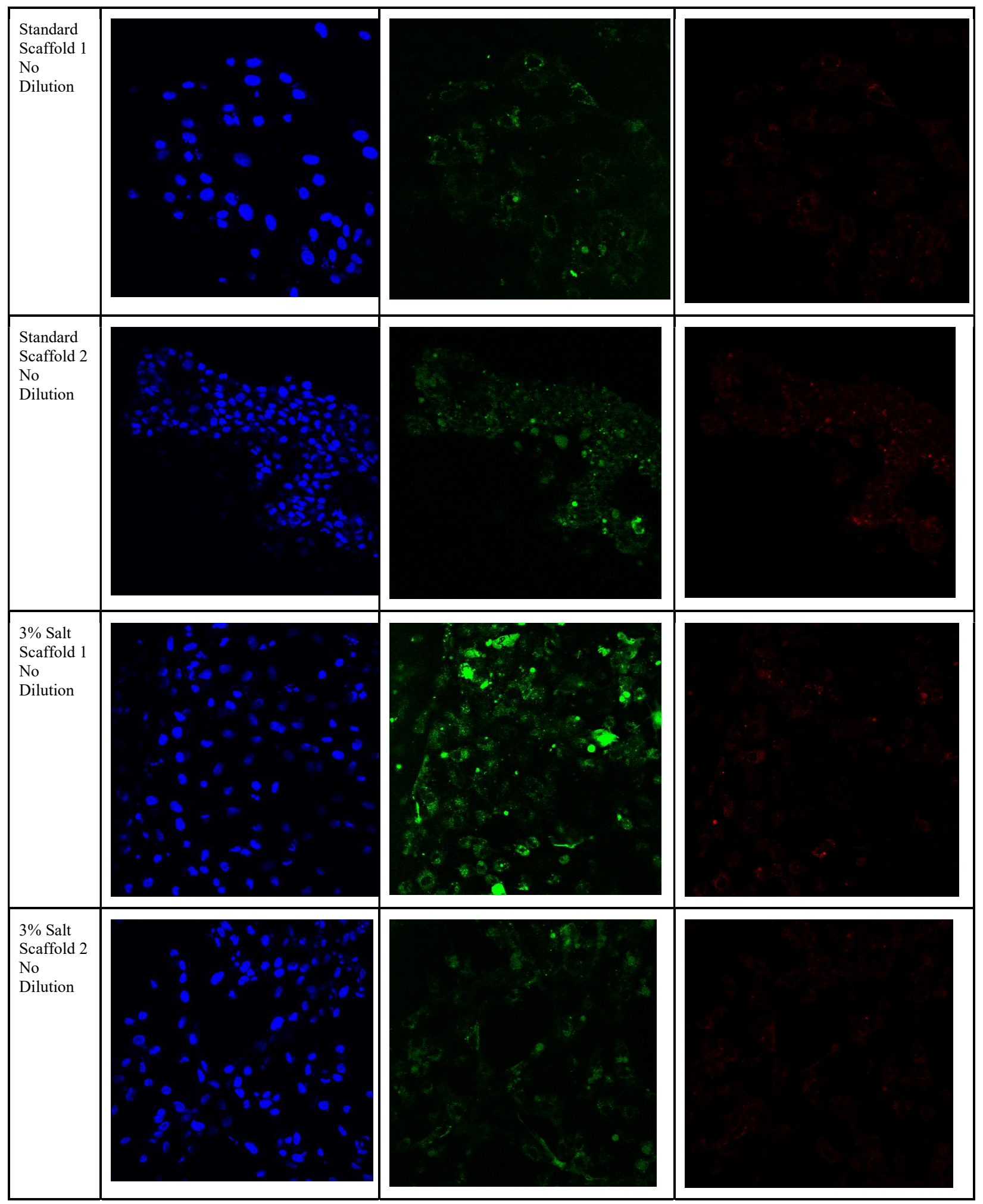




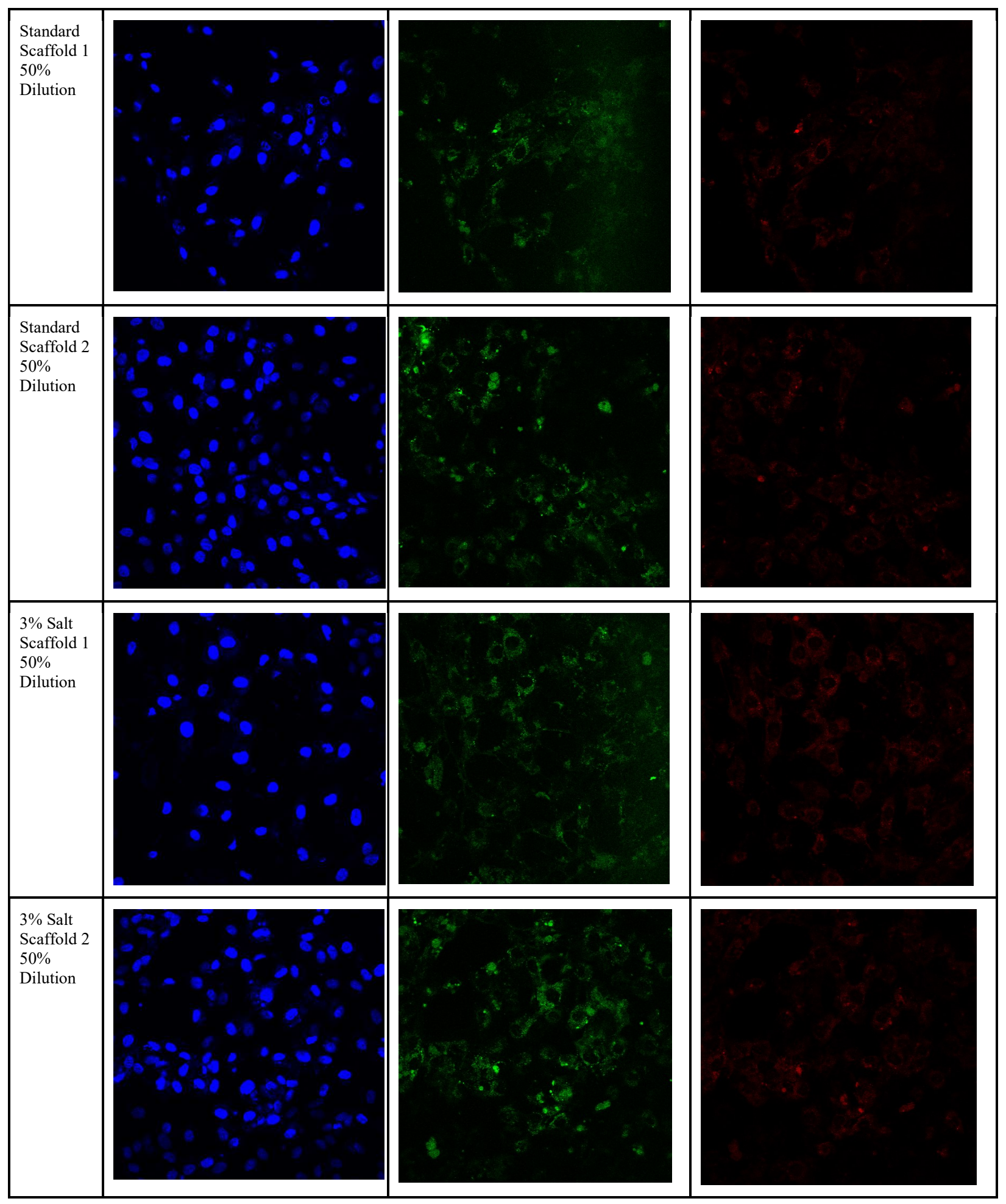

48 Hours, $40 x$

\begin{tabular}{|l|l|l|l|}
\hline & Hoechst & Caspase & Propidium Iodide \\
\hline
\end{tabular}




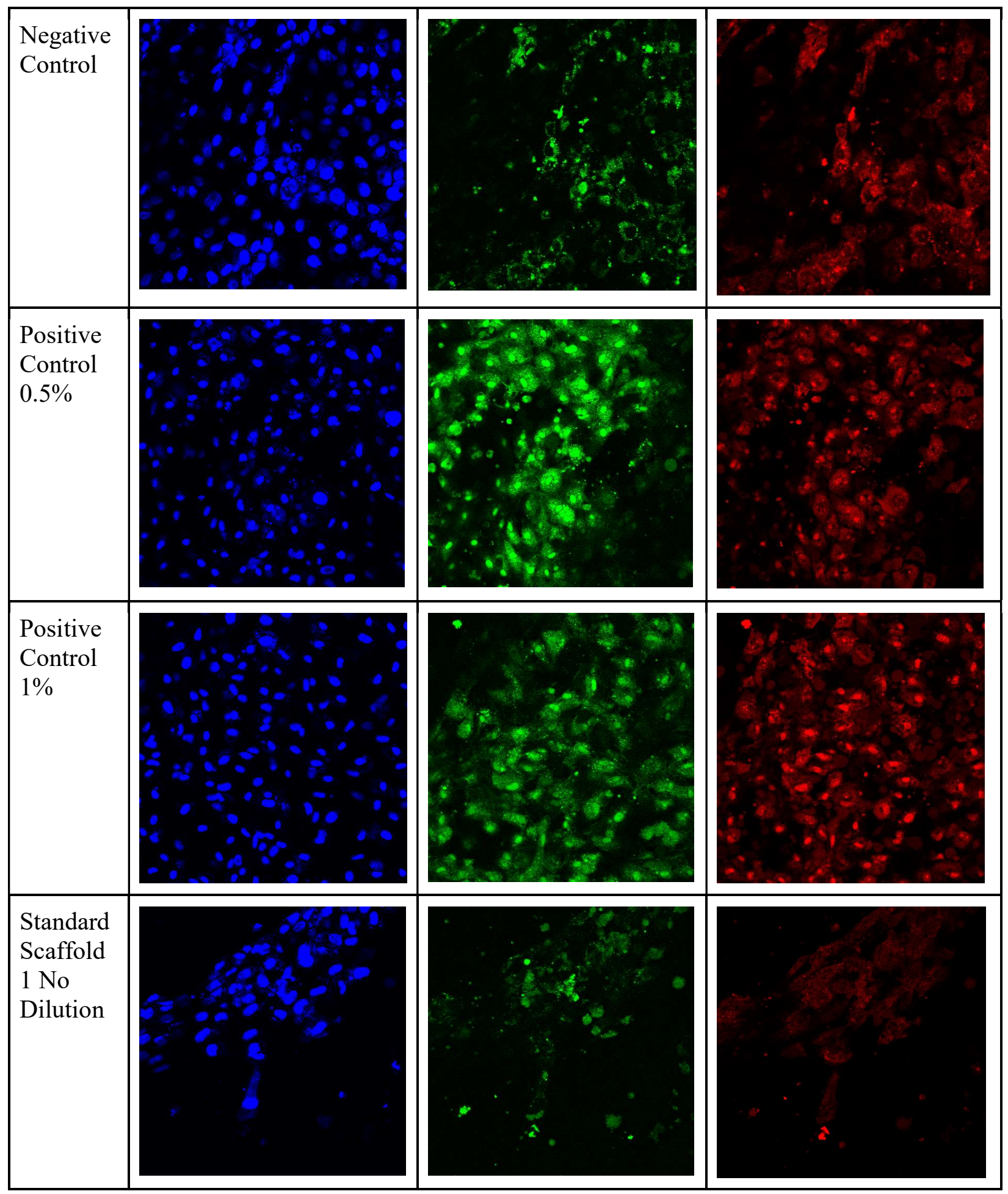




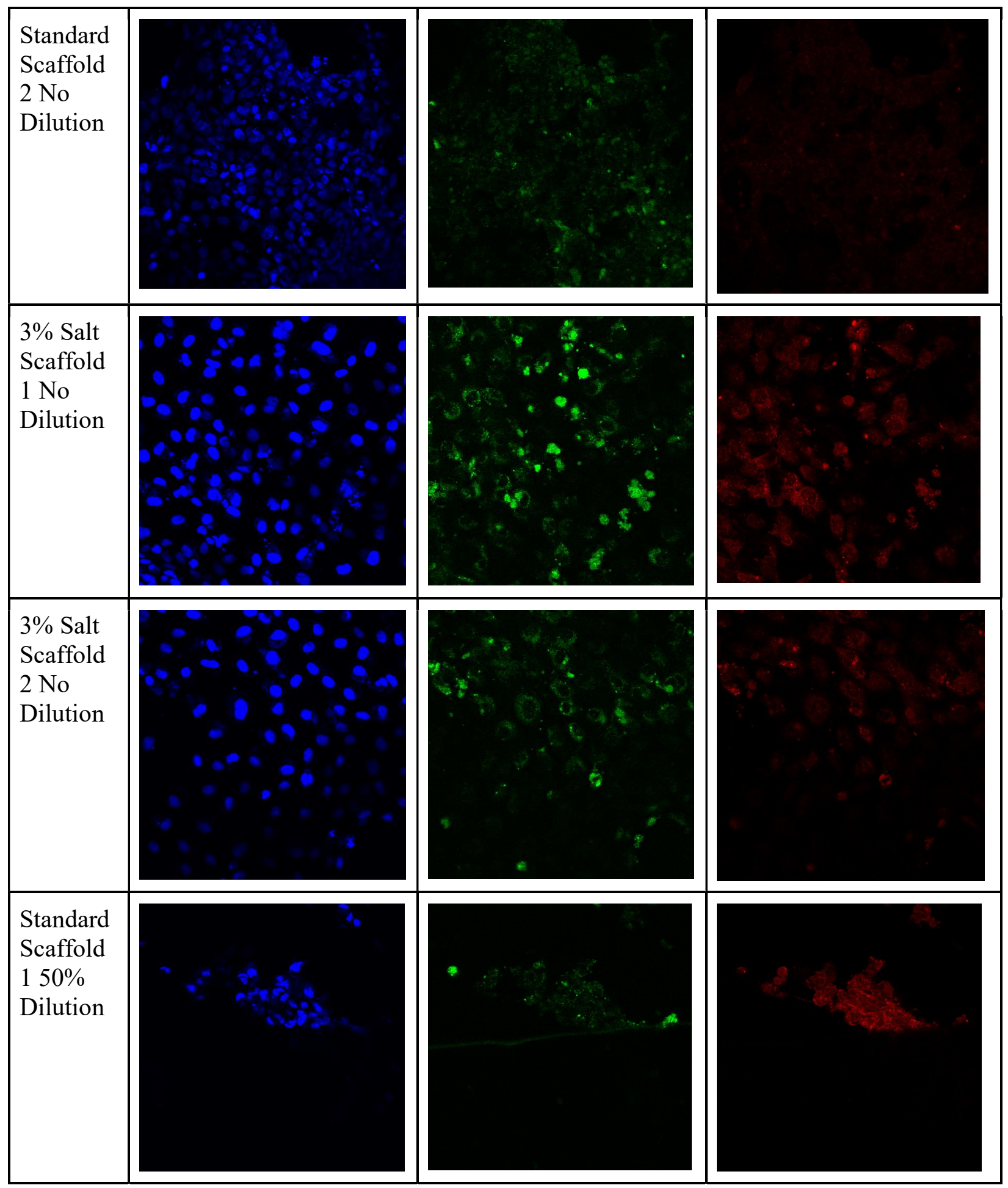




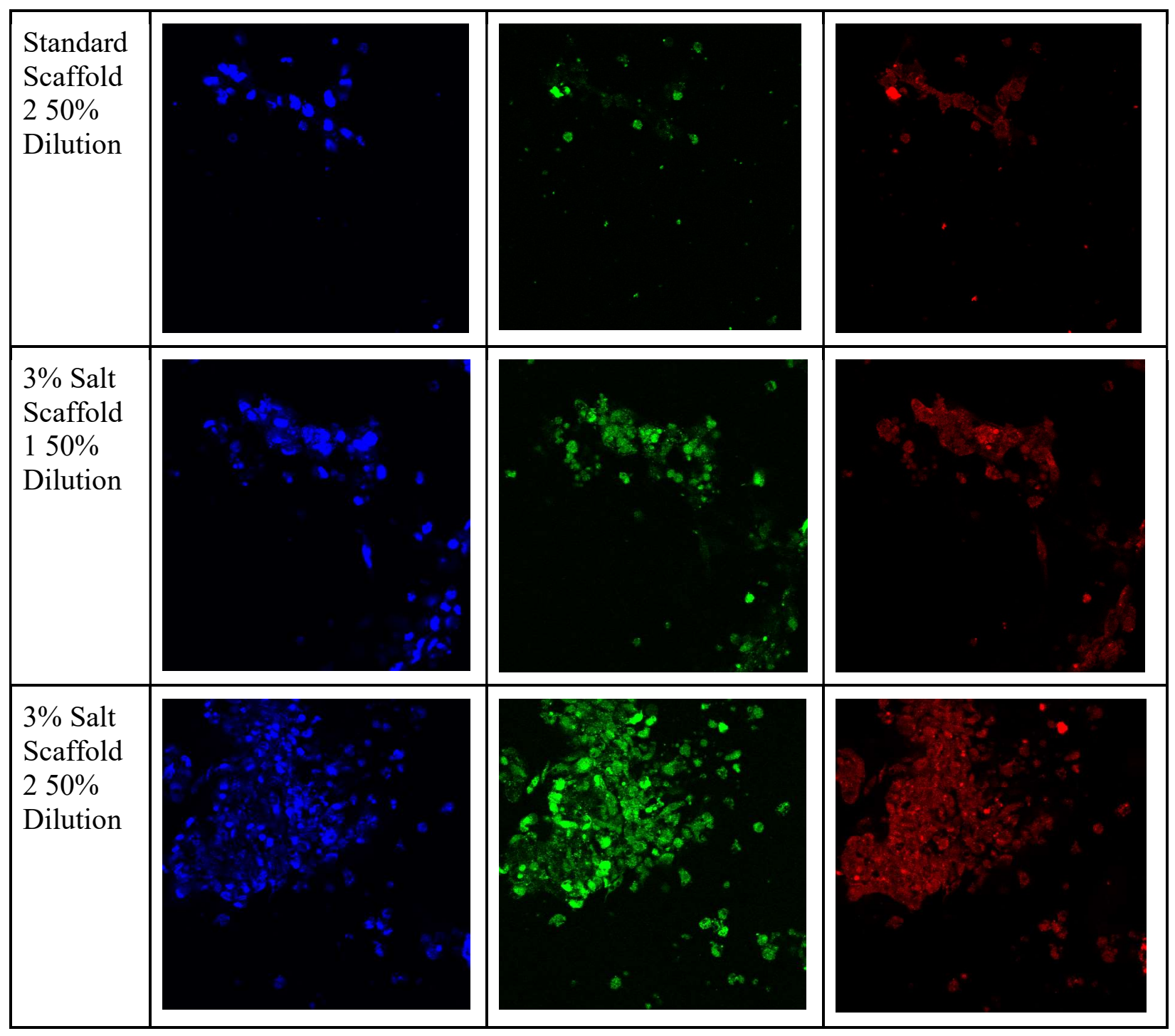


Appendix O: Scaffold Adhesion Assay Representative Images

\begin{tabular}{|c|c|c|c|}
\hline & Hoechst & Caspase & Propidium Iodide \\
\hline $\begin{array}{l}\text { Negative } \\
\text { Control } \\
\text { of } \\
\text { Standard } \\
\text { Scaffold }\end{array}$ & & & \\
\hline $\begin{array}{l}\text { Negative } \\
\text { Control } \\
\text { of Salt } \\
\text { Scaffold }\end{array}$ & & & \\
\hline $\begin{array}{l}\text { Standard } \\
\text { Scaffold } \\
1 \\
\text { Proximal }\end{array}$ & & & \\
\hline
\end{tabular}




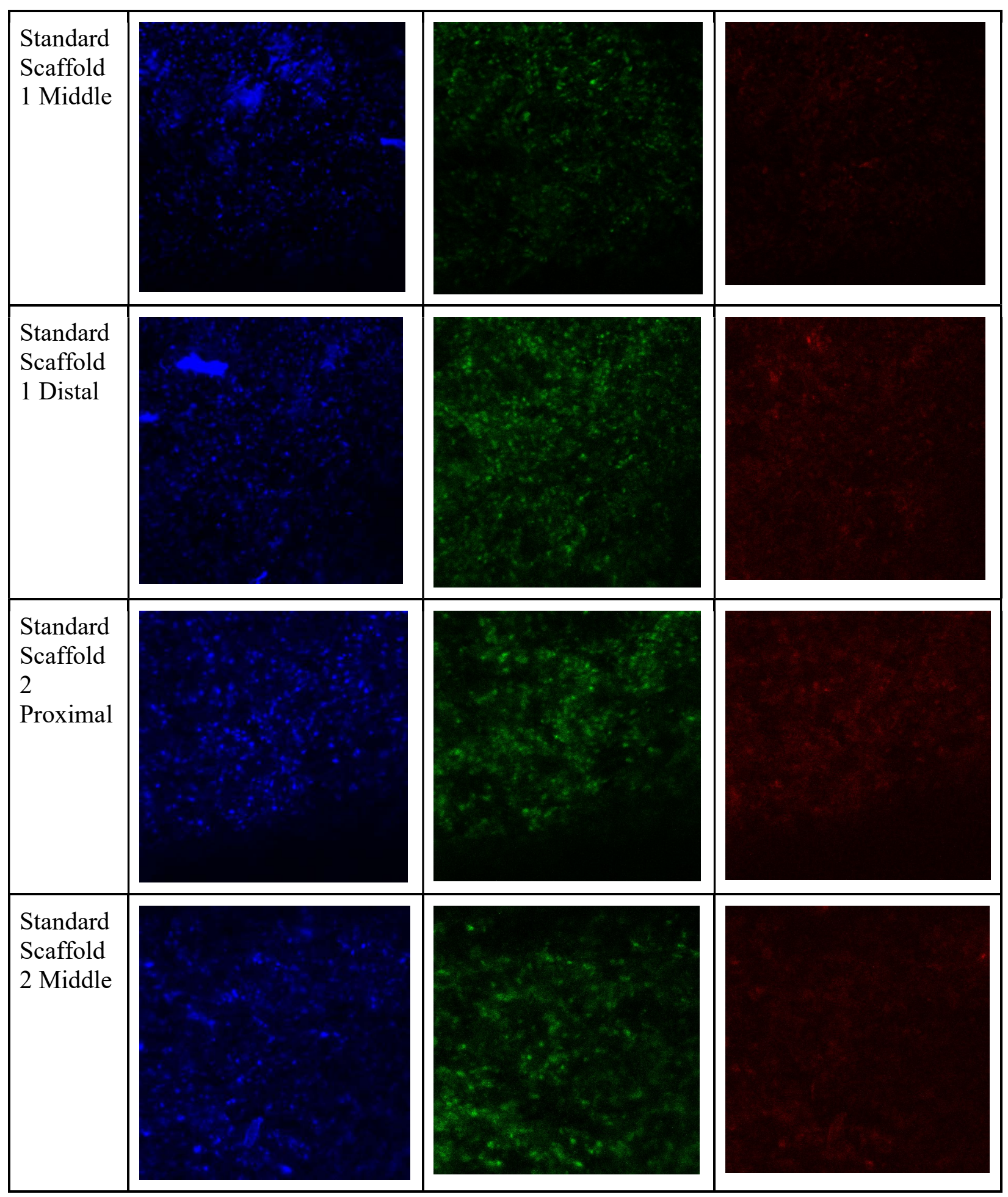




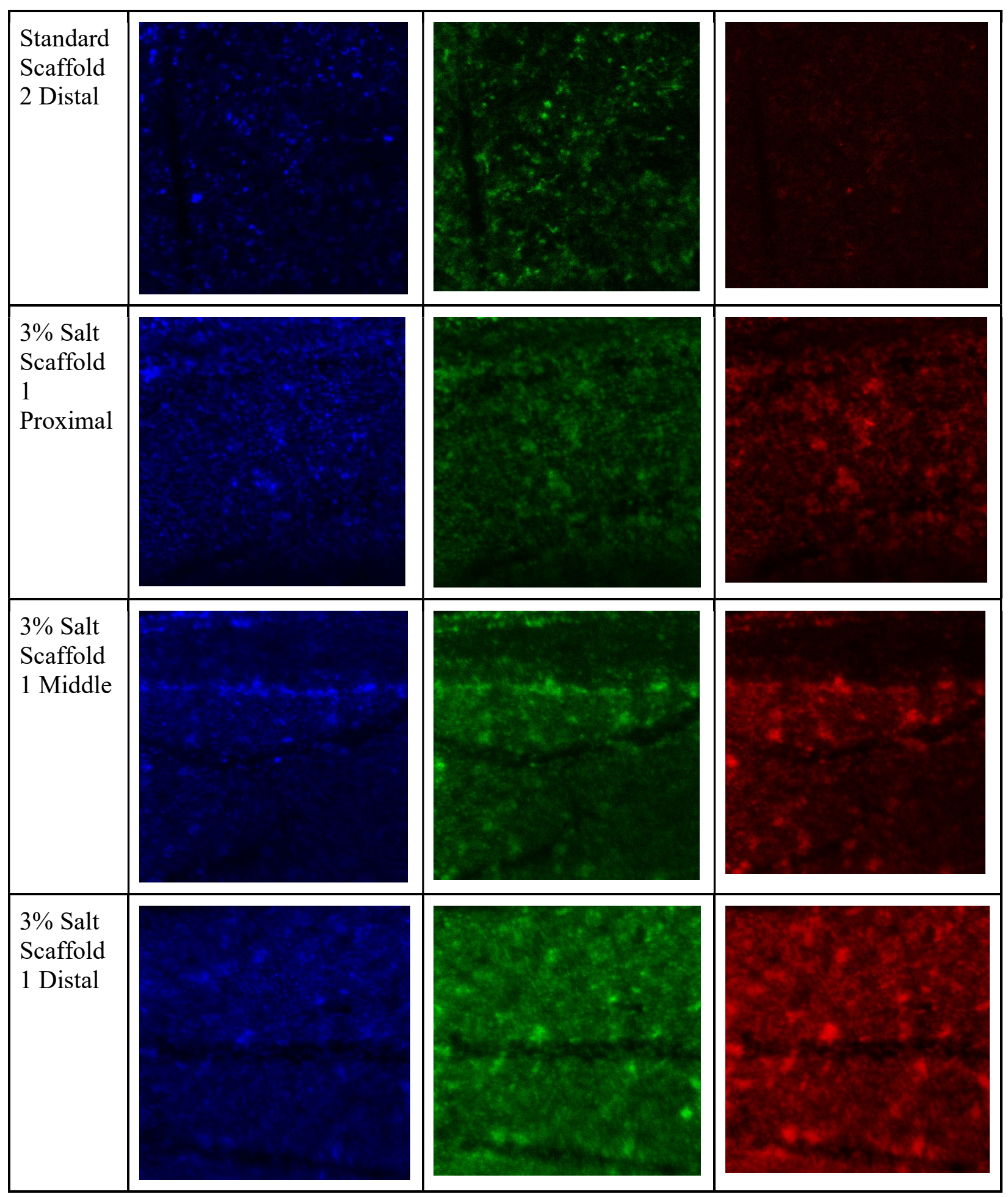




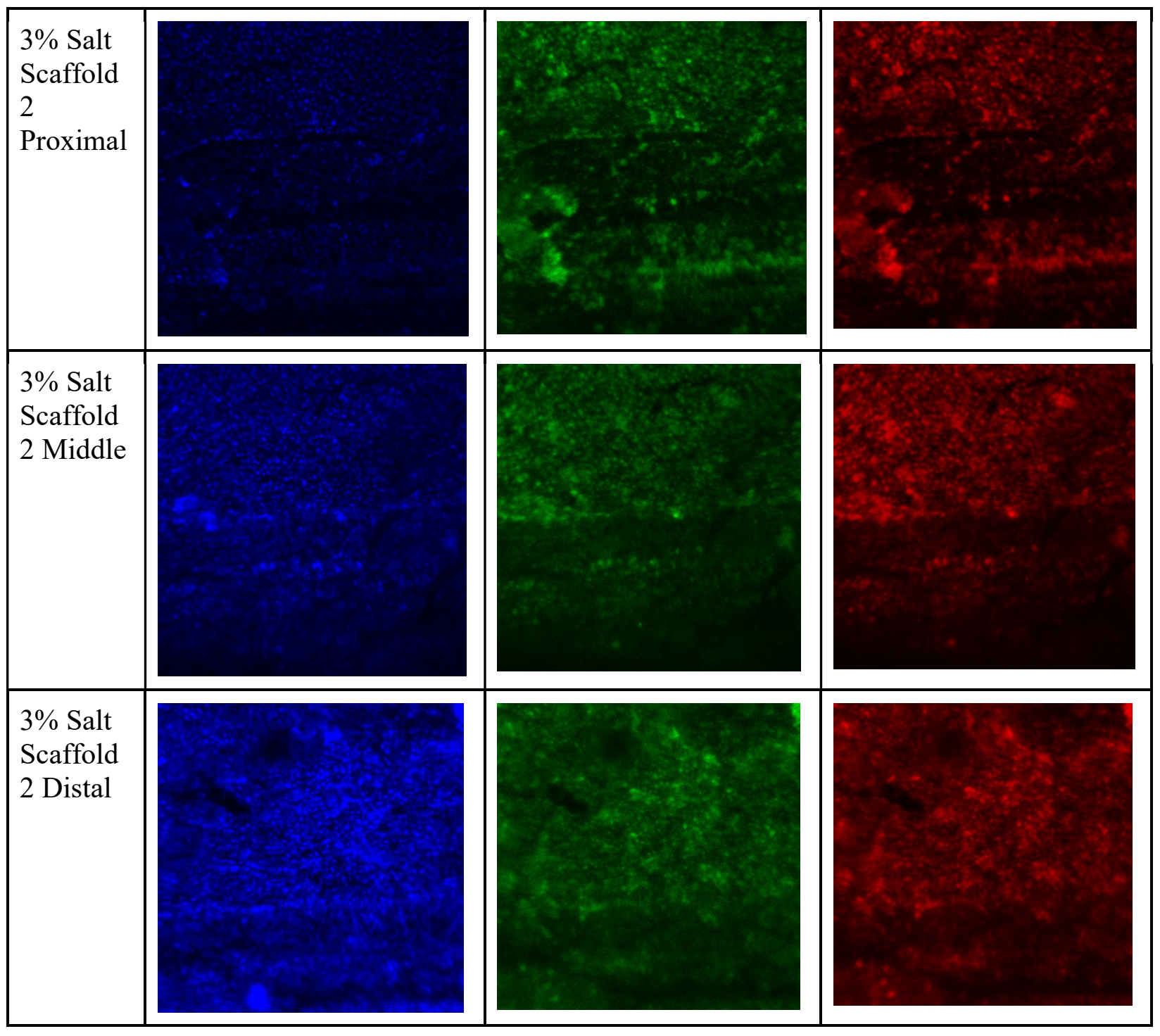


Appendix P: BVM Study Representative Images

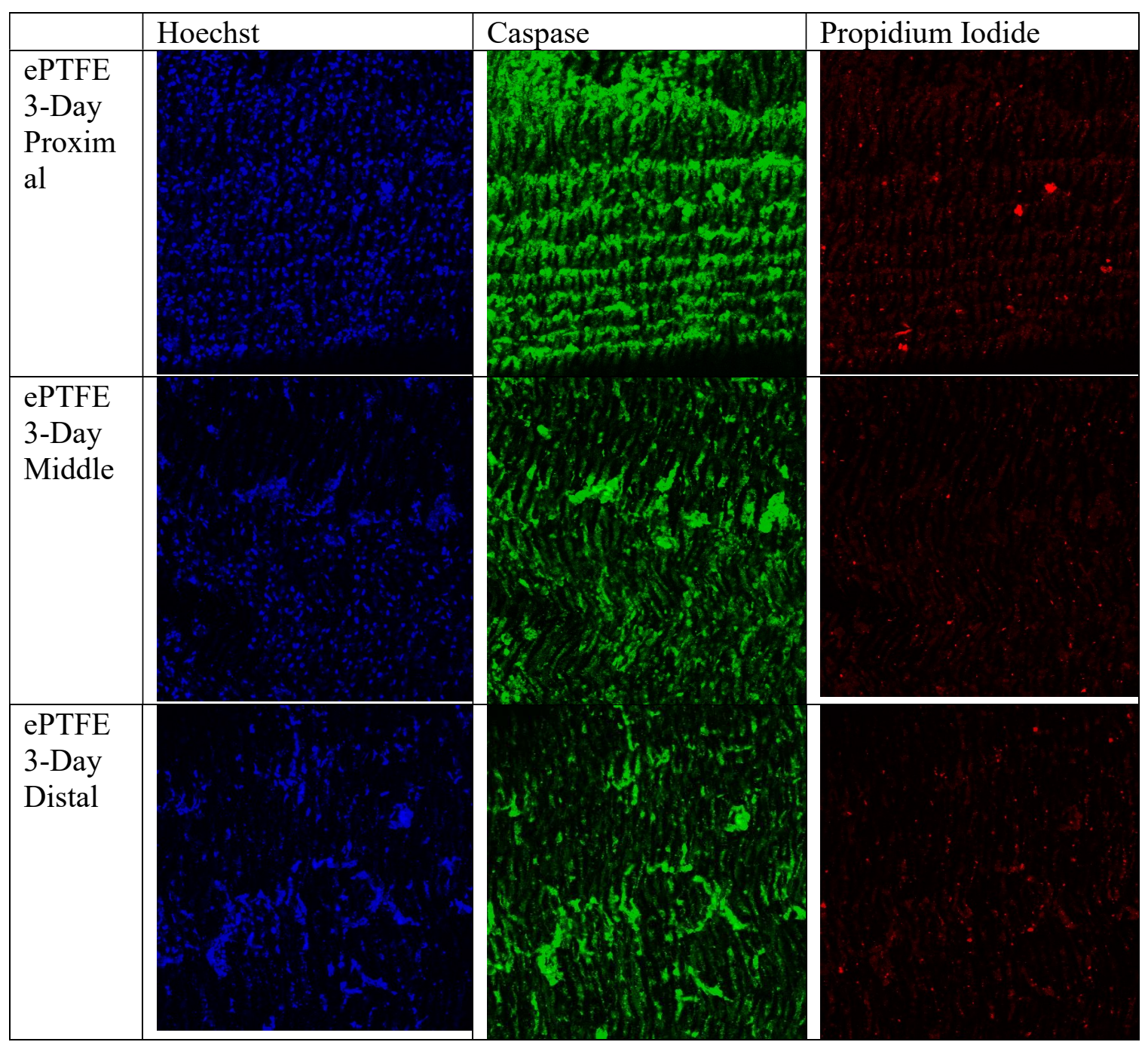




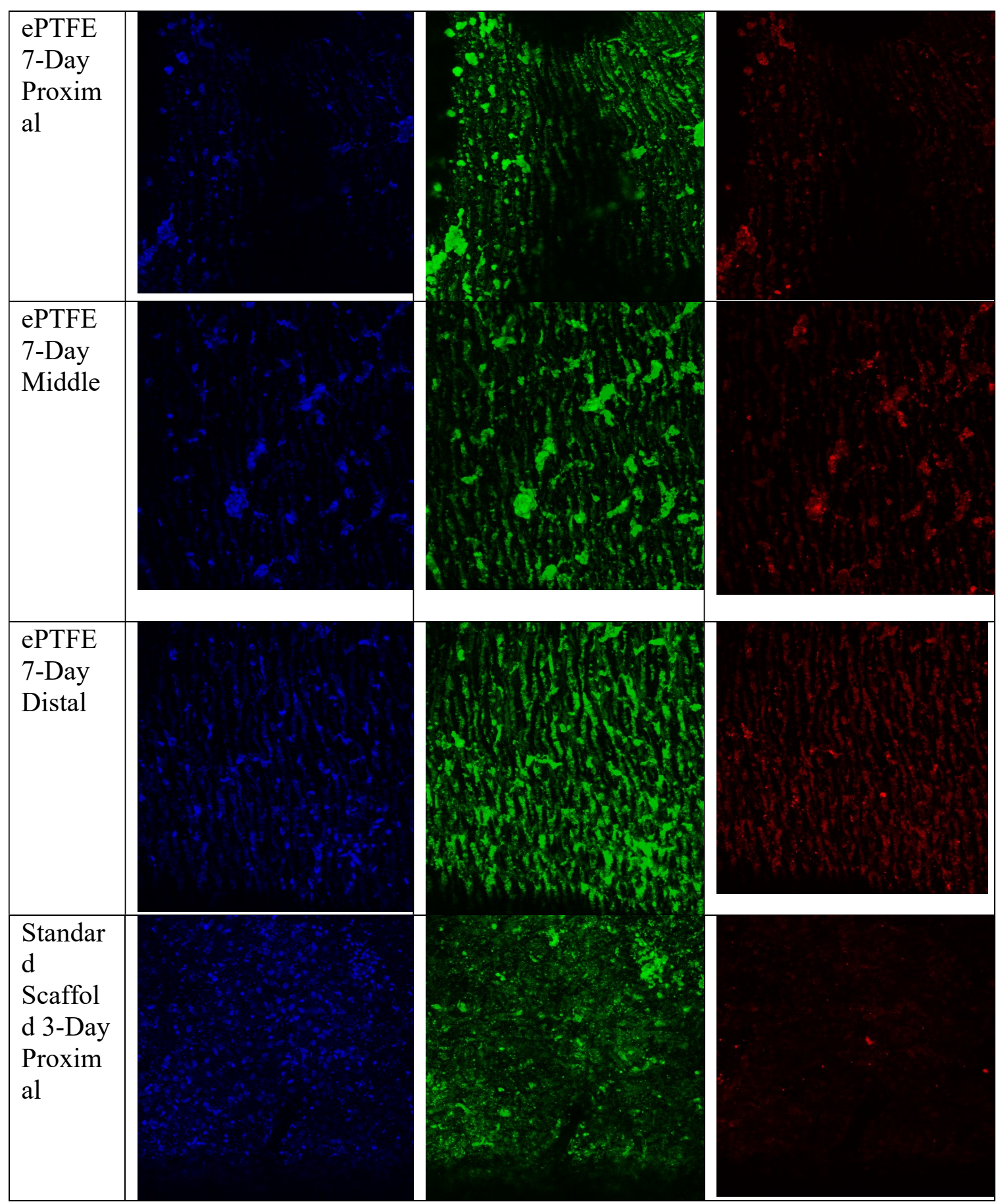




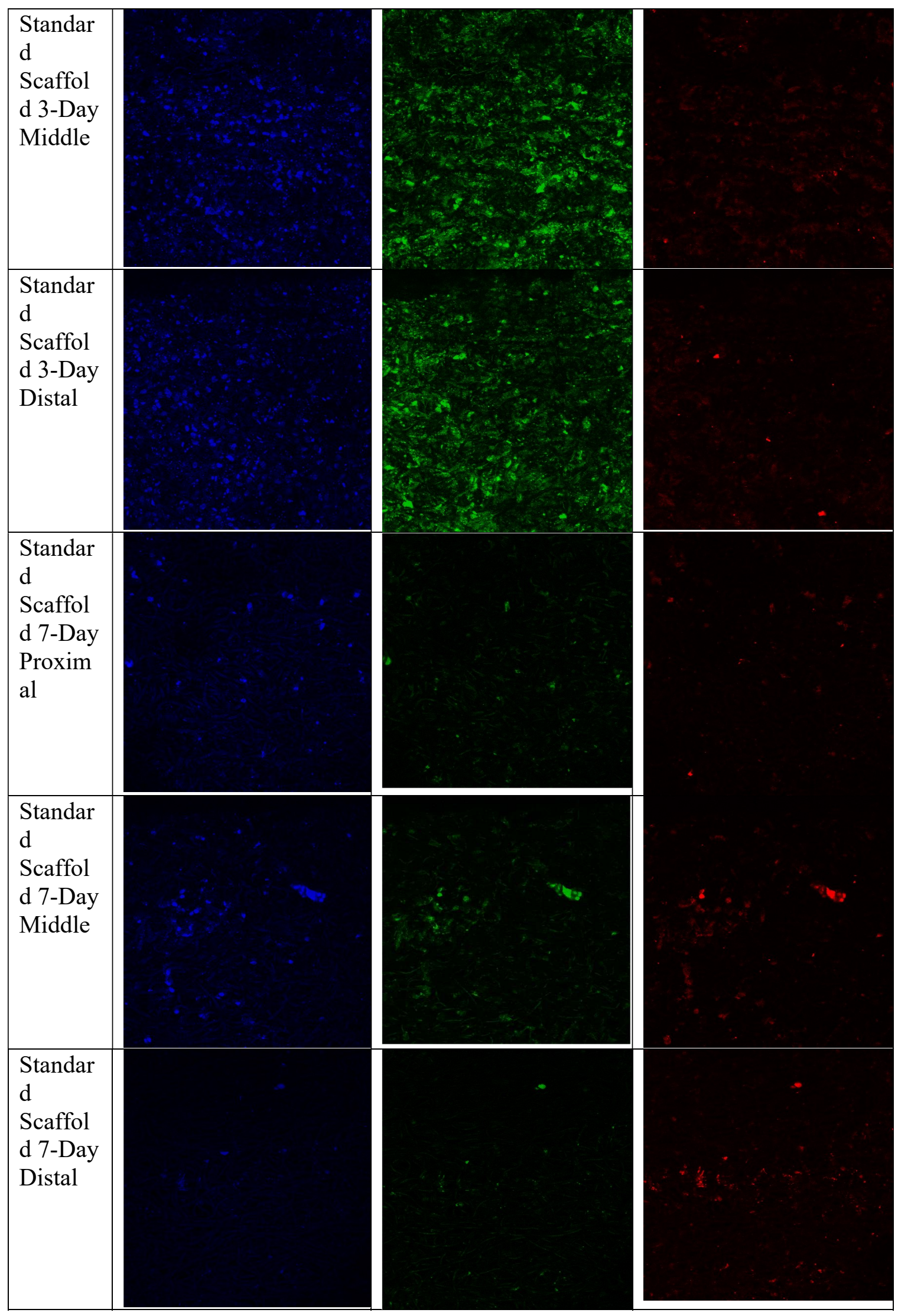




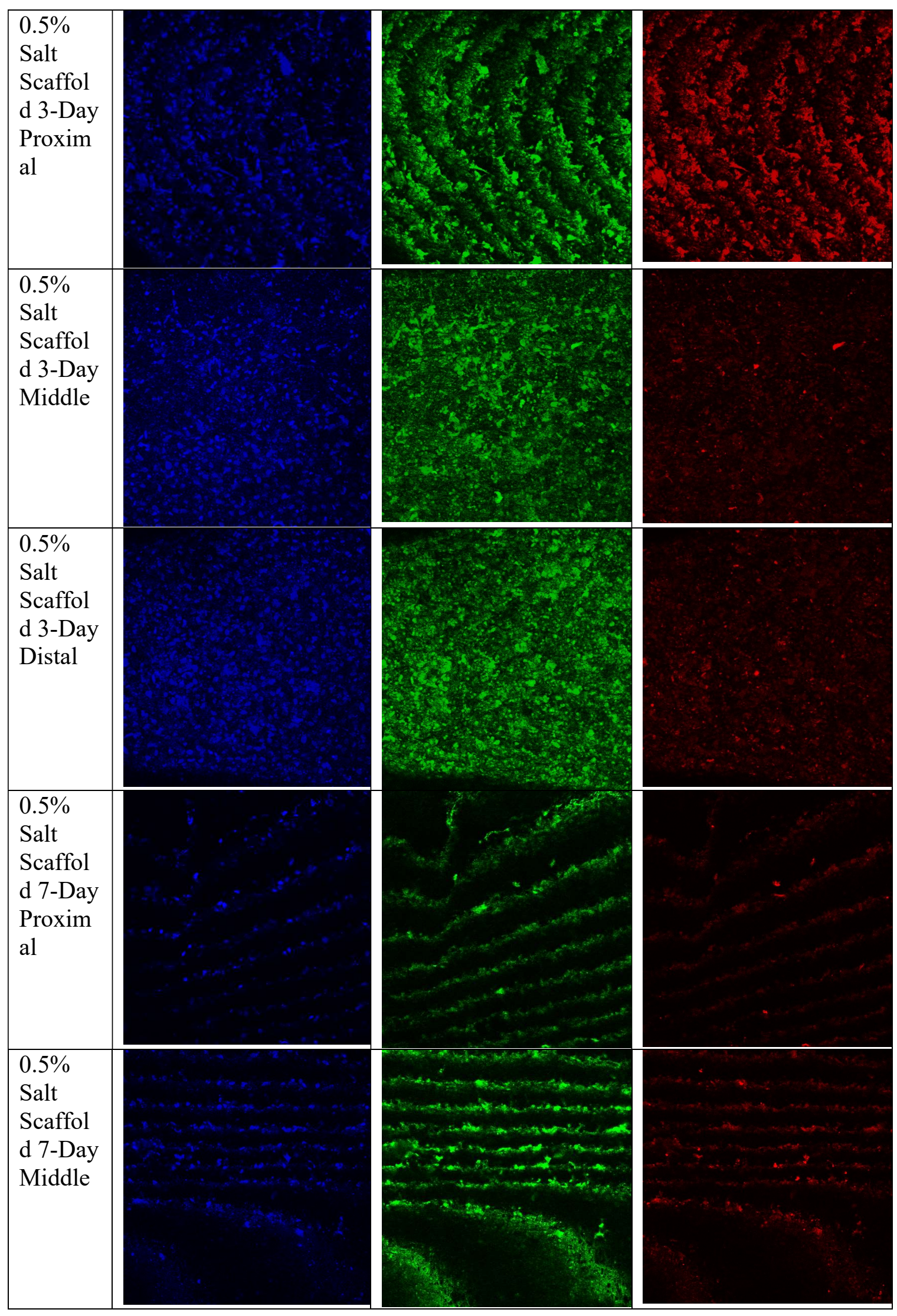




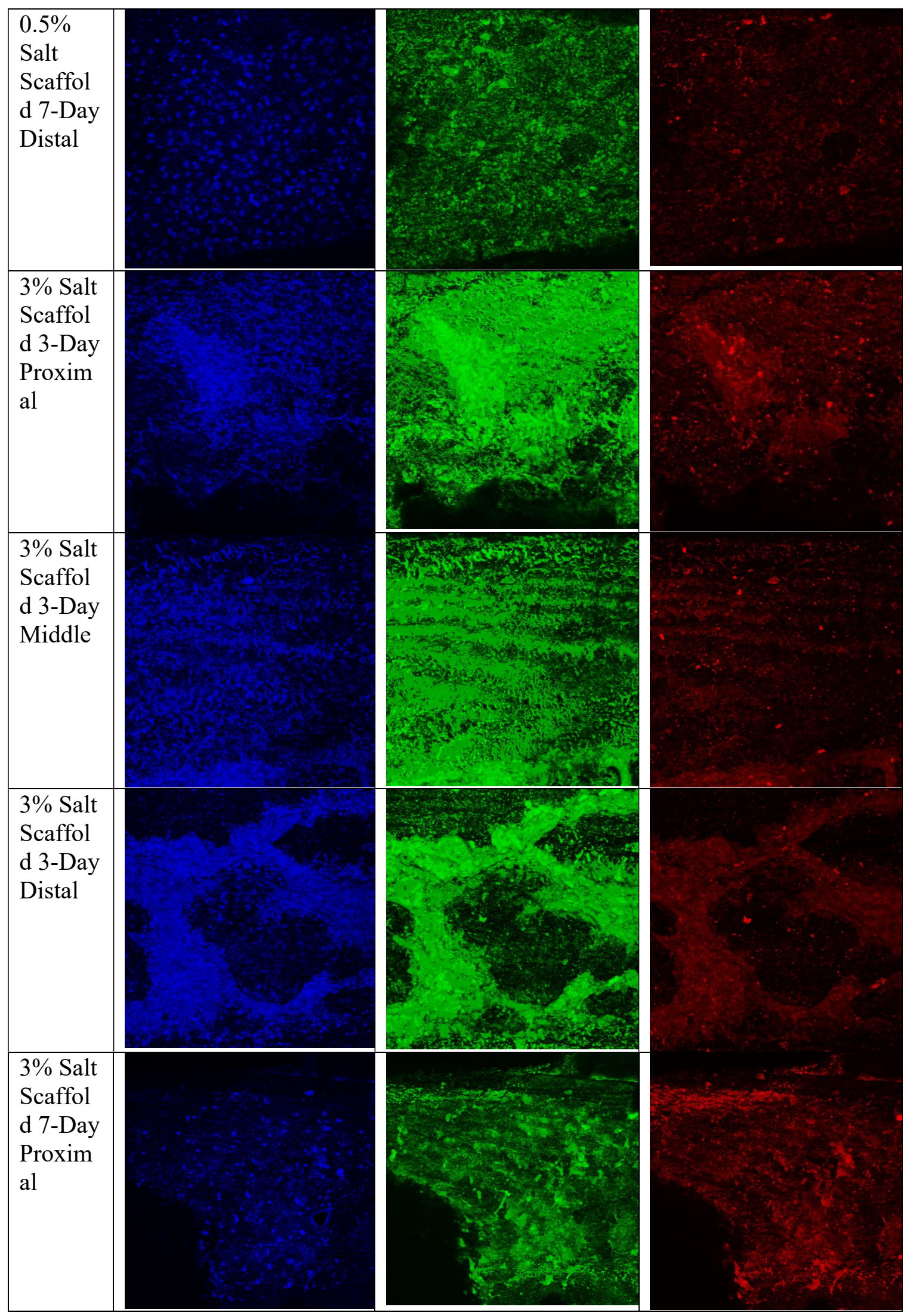




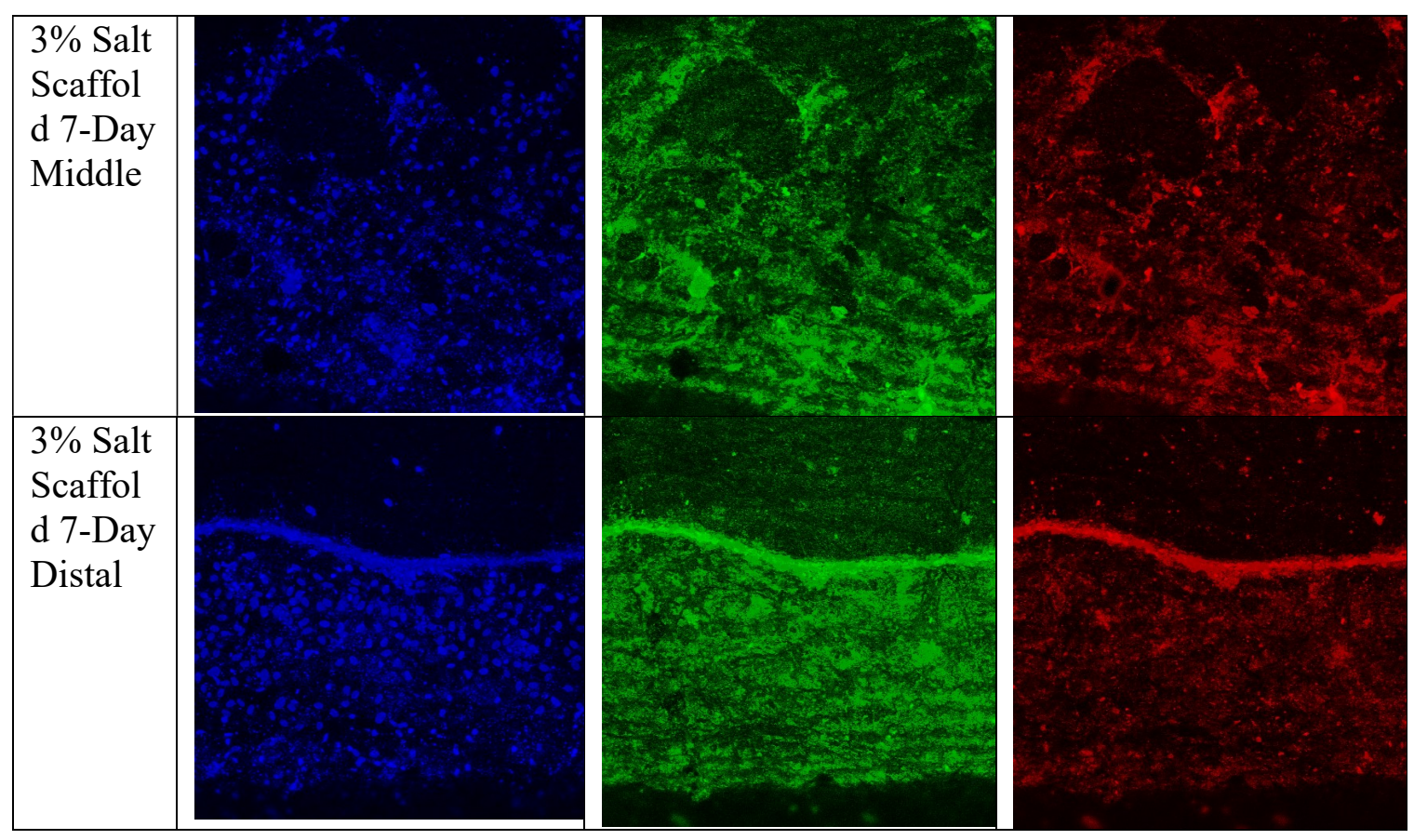




\section{Appendix Q: Magnification Justification Raw Data}

\section{Cytotoxicity 24 Hours 40x}

\begin{tabular}{|c|c|c|c|c|c|c|c|c|}
\hline $\begin{array}{l}\text { Image } \\
\text { Name }\end{array}$ & $\begin{array}{l}\text { Manual } \\
\text { Cell } \\
\text { Count }\end{array}$ & $\begin{array}{l}\text { Automated } \\
\text { Cell Count }\end{array}$ & $\begin{array}{l}\text { Difference } \\
\text { (Automated- } \\
\text { Manual) }\end{array}$ & $\begin{array}{l}\text { Threshold } \\
\text { (1st slider) }\end{array}$ & $\begin{array}{l}\text { Smallest } \\
\text { Cell Size } \\
\text { (pizels^2) }\end{array}$ & $\begin{array}{l}\text { Cell } \\
\text { Density }\end{array}$ & Notes & $\begin{array}{l}\text { Percent } \\
\text { Error }\end{array}$ \\
\hline $\begin{array}{l}\text { No } \\
\text { Dilution } \\
\text { No Salt } 1 \\
40 x 1\end{array}$ & 44 & 34 & -10 & 863 & 20 & Medium & & 29.41176471 \\
\hline $\begin{array}{l}\text { No } \\
\text { Dilution } \\
\text { No Salt } 1 \\
40 \times 2\end{array}$ & 57 & 59 & 2 & 1371 & 30 & Low & & 3.389830508 \\
\hline $\begin{array}{l}\text { No } \\
\text { Dilution } \\
\text { No Salt } 2 \\
40 \times 1\end{array}$ & 72 & 64 & -8 & 1776 & 30 & High & & 12.5 \\
\hline $\begin{array}{l}\text { No } \\
\text { Dilution } \\
\text { No Salt } 2 \\
40 \times 2\end{array}$ & 46 & 43 & -3 & 1390 & 30 & Medium & & 6.976744186 \\
\hline $\begin{array}{l}\text { No } \\
\text { Dilution } \\
\text { No Salt } 2 \\
40 \times 3\end{array}$ & 16 & 18 & 2 & 1466 & 30 & Low & & 11.11111111 \\
\hline $\begin{array}{l}\text { No } \\
\text { Dilution } \\
\text { No Salt } 2 \\
40 \times 4\end{array}$ & 22 & 24 & 2 & 1729 & 50 & Low & $\begin{array}{l}\text { Lots of } \\
\text { cell } \\
\text { debris }\end{array}$ & 8.333333333 \\
\hline $\begin{array}{l}\text { No } \\
\text { Dilution } \\
\text { Salt 1 } \\
40 x 1\end{array}$ & 68 & 53 & -15 & 1070 & 50 & Medium & & 28.30188679 \\
\hline $\begin{array}{l}\text { No } \\
\text { Dilution } \\
\text { Salt } 1 \\
40 \times 2\end{array}$ & 68 & 70 & 2 & 1546 & 30 & Low & & 2.857142857 \\
\hline
\end{tabular}




\begin{tabular}{|c|c|c|c|c|c|c|c|c|}
\hline $\begin{array}{l}\text { No } \\
\text { Dilution } \\
\text { Salt 1 } \\
\text { 40x } 3\end{array}$ & 30 & 30 & 0 & 1005 & 50 & Low & & 0 \\
\hline $\begin{array}{l}\text { No } \\
\text { Dilution } \\
\text { Salt 1 } \\
\text { 40x } 4\end{array}$ & 101 & 111 & 10 & 1070 & 50 & Medium & & 9.009009009 \\
\hline $\begin{array}{l}\text { No } \\
\text { Dilution } \\
\text { Salt 1 } \\
40 x 5\end{array}$ & 105 & 111 & 6 & 1101 & 50 & Medium & & 5.405405405 \\
\hline $\begin{array}{l}\text { No } \\
\text { Dilution } \\
\text { Salt } 2 \\
40 x 1\end{array}$ & 110 & 123 & 13 & 1031 & 50 & Medium & & 10.56910569 \\
\hline $\begin{array}{l}\text { No } \\
\text { Dilution } \\
\text { Salt } 2 \\
40 \times 2\end{array}$ & 108 & 111 & 3 & 1014 & 50 & Medium & & 2.702702703 \\
\hline $\begin{array}{l}\text { No } \\
\text { Dilution } \\
\text { Salt } 2 \\
40 x 3\end{array}$ & 28 & 30 & 2 & 1009 & 50 & Low & & 6.6666666667 \\
\hline $\begin{array}{l}\text { No } \\
\text { Dilution } \\
\text { Salt } 2 \\
40 x 4\end{array}$ & 40 & 40 & 0 & 1000 & 50 & Low & & 0 \\
\hline & & & & & & & $\begin{array}{l}\text { Average } \\
\text { Error }\end{array}$ & 9.148980198 \\
\hline
\end{tabular}

Cytotoxicity 24 hours $20 x$

\begin{tabular}{|c|c|c|c|c|c|c|c|c|}
\hline $\begin{array}{l}\text { Image } \\
\text { Name }\end{array}$ & $\begin{array}{l}\text { Manual } \\
\text { Cell } \\
\text { Count }\end{array}$ & $\begin{array}{l}\text { Automated } \\
\text { Cell Count }\end{array}$ & $\begin{array}{l}\text { Difference } \\
\text { (Automated- } \\
\text { Manual) }\end{array}$ & $\begin{array}{l}\text { Threshold } \\
\text { (1st slider) }\end{array}$ & $\begin{array}{l}\text { Smallest } \\
\text { Cell Size } \\
(\text { pizels^2) }\end{array}$ & $\begin{array}{l}\text { Cell } \\
\text { Density }\end{array}$ & Notes & Error \\
\hline $\begin{array}{l}\text { No Salt } \\
120 x 1\end{array}$ & 17 & 17 & 0 & 1009 & 20 & Low & & 0 \\
\hline $\begin{array}{l}\text { No Salt } \\
120 \times 2 \\
\end{array}$ & 42 & 37 & 5 & 1334 & 20 & Medium & & 13.51351351 \\
\hline $\begin{array}{l}\text { No Salt } \\
120 \times 3 \\
\end{array}$ & 31 & 23 & 8 & 1008 & 20 & Low & & 34.7826087 \\
\hline $\begin{array}{l}\text { No Salt } \\
120 \mathrm{x} 4 \\
\end{array}$ & 81 & 43 & 38 & 1009 & 20 & High & & 88.37209302 \\
\hline
\end{tabular}




\begin{tabular}{|c|c|c|c|c|c|c|c|c|}
\hline $\begin{array}{l}\text { No Salt } \\
220 \times 1\end{array}$ & 127 & 73 & 54 & 1102 & 20 & Medium & & 73.97260274 \\
\hline $\begin{array}{l}\text { No Salt } \\
220 \times 2\end{array}$ & 22 & 20 & 2 & 1084 & 20 & Low & & 10 \\
\hline $\begin{array}{l}\text { No Salt } \\
220 \times 3\end{array}$ & 100 & 101 & -1 & 1101 & 20 & Medium & & 0.9900990099 \\
\hline $\begin{array}{l}\text { No Salt } \\
220 \times 4\end{array}$ & 101 & 64 & 37 & 1181 & 20 & High & & 57.8125 \\
\hline $\begin{array}{l}\text { No Salt } \\
220 \times 5\end{array}$ & 120 & 90 & 30 & 1087 & 20 & High & & 33.33333333 \\
\hline $\begin{array}{l}\text { Salt } 1 \\
20 x 1\end{array}$ & 49 & 22 & 27 & 1087 & 20 & Medium & & 122.7272727 \\
\hline $\begin{array}{l}\text { Salt } 1 \\
20 x 2\end{array}$ & 55 & 35 & 20 & 1060 & 20 & High & & 57.14285714 \\
\hline $\begin{array}{l}\text { Salt } 1 \\
20 x 4\end{array}$ & 84 & 67 & 17 & 811 & 20 & Medium & & 25.37313433 \\
\hline $\begin{array}{l}\text { Salt } 2 \\
20 \times 1\end{array}$ & 96 & 82 & 14 & 1149 & 20 & Low & & 17.07317073 \\
\hline $\begin{array}{l}\text { Salt } 2 \\
20 x 2\end{array}$ & 74 & 79 & -5 & 1027 & 20 & Medium & & 6.329113924 \\
\hline $\begin{array}{l}\text { Salt } 2 \\
20 x 3\end{array}$ & 59 & 51 & 8 & 1009 & 20 & Medium & & 15.68627451 \\
\hline $\begin{array}{l}\text { Salt } 2 \\
20 x 4\end{array}$ & 66 & 36 & 30 & 1227 & 20 & Low & & 83.33333333 \\
\hline & & & & & & & Average & 40.02761919 \\
\hline
\end{tabular}

\section{Appendix R: Pixel Intensity Reduction Raw Data}

\begin{tabular}{|l|r|r|l|r|}
\hline $\begin{array}{l}\text { Propidium } \\
\text { Iodide }\end{array}$ & $\begin{array}{l}\text { Min } \\
\text { Intensity }\end{array}$ & $\begin{array}{l}\text { Cell } \\
\text { Count }\end{array}$ & $\begin{array}{l}\text { Percent Cell Count } \\
\text { Reduction }\end{array}$ & \multicolumn{2}{l|}{$\begin{array}{l}\text { Average Percent Cell Count } \\
\text { Reduction }\end{array}$} \\
\hline & 0 & 22 & 0 & 0 \\
\hline & 5 & 21 & 4.545454545 & 1.515151515 \\
\hline & 10 & 21 & 4.545454545 & 6.62701022 \\
\hline & 15 & 21 & 4.545454545 & 10.798402648 \\
\hline & 20 & 18 & 18.18181818 & 20.08181054 \\
\hline & 25 & 15 & 31.81818182 & 27.17715422 \\
\hline
\end{tabular}




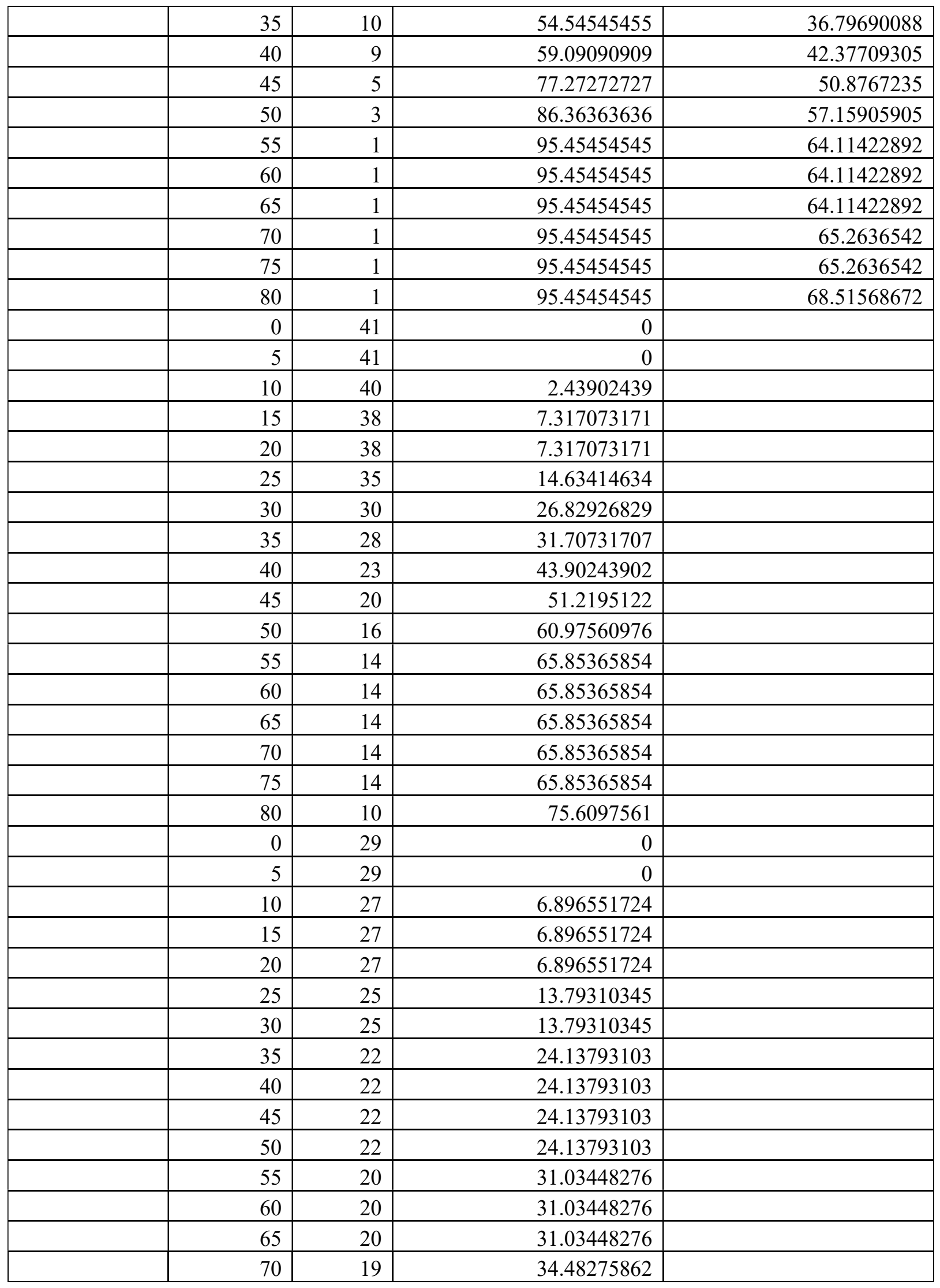




\begin{tabular}{|c|c|c|c|c|}
\hline & 75 & 19 & 34.48275862 & \\
\hline & 80 & 19 & 34.48275862 & \\
\hline Caspase & $\begin{array}{l}\text { Min } \\
\text { Intensity }\end{array}$ & $\begin{array}{l}\text { Cell } \\
\text { Count }\end{array}$ & $\begin{array}{l}\text { Percent Cell Count } \\
\text { Reduction }\end{array}$ & $\begin{array}{l}\text { Average Percent Cell Count } \\
\text { Reduction }\end{array}$ \\
\hline & 0 & 22 & 0 & 0 \\
\hline & 5 & 22 & 0 & 0 \\
\hline & 10 & 21 & 4.545454545 & 2.727272727 \\
\hline & 15 & 18 & 18.18181818 & 10.71566731 \\
\hline & 20 & 15 & 31.81818182 & 17.38878143 \\
\hline & 25 & 15 & 31.81818182 & 17.38878143 \\
\hline & 30 & 12 & 45.45454545 & 26.48613798 \\
\hline & 35 & 11 & 50 & 28.6073501 \\
\hline & 40 & 11 & 50 & 29.81947131 \\
\hline & 45 & 11 & 50 & 37.81431335 \\
\hline & 50 & 11 & 50 & 37.81431335 \\
\hline & 55 & 8 & 63.63636364 & 43.77820761 \\
\hline & 60 & 6 & 72.72727273 & 52.88201161 \\
\hline & 65 & 6 & 72.72727273 & 55.00967118 \\
\hline & 70 & 6 & 72.72727273 & 56.4281109 \\
\hline & 75 & 4 & 81.81818182 & 64.61637653 \\
\hline & 80 & 3 & 86.36363636 & 67.34364926 \\
\hline & 0 & 55 & 0 & \\
\hline & 5 & 55 & 0 & \\
\hline & 10 & 53 & 3.636363636 & \\
\hline & 15 & 52 & 5.454545455 & \\
\hline & 20 & 52 & 5.454545455 & \\
\hline & 25 & 52 & 5.454545455 & \\
\hline & 30 & 48 & 12.72727273 & \\
\hline & 35 & 47 & 14.54545455 & \\
\hline & 40 & 45 & 18.18181818 & \\
\hline & 45 & 40 & 27.27272727 & \\
\hline & 50 & 40 & 27.27272727 & \\
\hline & 55 & 40 & 27.27272727 & \\
\hline & 60 & 37 & 32.72727273 & \\
\hline & 65 & 37 & 32.72727273 & \\
\hline & 70 & 37 & 32.72727273 & \\
\hline & 75 & 32 & 41.81818182 & \\
\hline & 80 & 30 & 45.45454545 & \\
\hline & 0 & 47 & 0 & \\
\hline & 5 & 47 & 0 & \\
\hline & 10 & 47 & 0 & \\
\hline & 15 & 43 & 8.510638298 & \\
\hline
\end{tabular}




\begin{tabular}{|l|l|l|l|l|}
\hline & 20 & 40 & 14.89361702 & \\
\hline & 25 & 40 & 14.89361702 & \\
\hline & 30 & 37 & 21.27659574 & \\
\hline & 35 & 37 & 21.27659574 & \\
\hline & 40 & 37 & 21.27659574 & \\
\hline & 45 & 30 & 36.17021277 & \\
\hline & 50 & 30 & 36.17021277 & \\
\hline & 55 & 28 & 40.42553191 & \\
\hline & 60 & 22 & 53.19148936 & \\
\hline & 65 & 19 & 59.57446809 & \\
\hline & 70 & 17 & 63.82978723 & \\
\hline & 75 & 14 & 70.21276596 & \\
\hline & 80 & 14 & 70.21276596 & \\
\hline
\end{tabular}

\section{Appendix S: Nuclei Area Multiplication Factor Raw Data}

\begin{tabular}{|r|r|r|l|r|}
\hline Cell & Nucleus Size & Whole Cell Size & Nucleus \% & Multiplication Factor \\
\hline 1 & 14 & 84 & 16.66666667 & 6 \\
\hline 2 & 29 & 209 & 13.87559809 & 7.206896552 \\
\hline 3 & 48 & 171 & 28.07017544 & 3.5625 \\
\hline 4 & 26 & 135 & 19.25925926 & 5.192307692 \\
\hline 5 & 38 & 137 & 27.73722628 & 3.605263158 \\
\hline 6 & 50 & 158 & 31.64556962 & 3.16 \\
\hline 7 & 44 & 84 & 52.38095238 & 1.909090909 \\
\hline
\end{tabular}




\begin{tabular}{|c|c|c|c|c|}
\hline 8 & 22 & 100 & 22 & 4.545454545 \\
\hline 9 & 44 & 105 & 41.9047619 & 2.386363636 \\
\hline 10 & 57 & 169 & 33.72781065 & 2.964912281 \\
\hline 11 & 27 & 141 & 19.14893617 & 5.222222222 \\
\hline 12 & 20 & 161 & 12.42236025 & 8.05 \\
\hline 13 & 24 & 110 & 21.81818182 & 4.583333333 \\
\hline 14 & 40 & 328 & 12.19512195 & 8.2 \\
\hline 15 & 26 & 173 & 15.02890173 & 6.653846154 \\
\hline 16 & 29 & 161 & 18.01242236 & 5.551724138 \\
\hline 17 & 52 & 245 & 21.2244898 & 4.711538462 \\
\hline 18 & 23 & 91 & 25.27472527 & 3.956521739 \\
\hline 19 & 29 & 158 & 18.35443038 & 5.448275862 \\
\hline 20 & 21 & 119 & 17.64705882 & 5.666666667 \\
\hline & & $\begin{array}{l}\text { Nucleus \% } \\
\text { Average }\end{array}$ & 23.41973244 & \\
\hline & & $\begin{array}{l}\text { Multiplication } \\
\text { Factor }\end{array}$ & 4.269903606 & \\
\hline
\end{tabular}

\section{Appendix T: Statistical Tests for Cytotoxicity Elution Assay}

ANOVA for Caspase Activity without normalizing to negative control 


\begin{tabular}{|l|r|r|r|r|}
\hline \multicolumn{3}{|c|}{ Analysis of Variance } \\
\hline Source & DF & $\begin{array}{r}\text { Sum of } \\
\text { Squares }\end{array}$ & Mean Square & F Ratio \\
\hline Model & 13 & 39209.060 & 3016.08 & 7.5080 \\
Error & 73 & 29325.301 & 401.72 & Prob > F \\
C. Total & 86 & 68534.361 & & $<.0001 *$
\end{tabular}

\begin{tabular}{l|rrrr}
\hline Parameter Estimates & & & & \\
\hline Term & Estimate & Std Error & t Ratio & Prob $>|\mathbf{t}|$ \\
\hline Intercept & 46.277194 & 2.263431 & 20.45 & $<.0001^{*}$ \\
Duration[24 Hours] & -7.074356 & 2.263431 & -3.13 & $0.0025^{*}$ \\
Treatment[Negative Control] & -27.12803 & 5.917156 & -4.58 & $<.0001^{*}$ \\
Treatment[Normal 50\% Dilution] & -18.48435 & 4.801759 & -3.85 & $0.0003^{*}$ \\
Treatment[Normal No Dilution] & -21.17104 & 5.238387 & -4.04 & $0.0001^{*}$ \\
Treatment[Positive Control 0.5\%] & 30.289234 & 6.402395 & 4.73 & $<.0001^{*}$ \\
Treatment[Positive Control 1\%] & 27.596974 & 6.402395 & 4.31 & $<.0001^{*}$ \\
Treatment[Salt 50\% Dilution] & 6.0206489 & 4.933343 & 1.22 & 0.2262 \\
Duration[24 Hours] ${ }^{*}$ Treatment[Negative Control] & 3.606543 & 5.917156 & 0.61 & 0.5441 \\
Duration[24 Hours]*Treatment[Normal 50\% Dilution] & 3.3408742 & 4.801759 & 0.70 & 0.4888 \\
Duration[24 Hours] ${ }^{*}$ Treatment[Normal No Dilution] & -0.312345 & 5.238387 & -0.06 & 0.9526 \\
Duration[24 Hours] ${ }^{*}$ Treatment[Positive Control 0.5\%] & -7.778947 & 6.402395 & -1.22 & 0.2283 \\
Duration[24 Hours] ${ }^{*}$ Treatment[Positive Control 1\%] & 10.86819 & 6.402395 & 1.70 & 0.0939 \\
Duration[24 Hours]*Treatment[Salt 50\% Dilution] & -9.687699 & 4.933343 & -1.96 & 0.0534
\end{tabular}

\begin{tabular}{|l|l|l|r}
\hline Level & & & $\begin{array}{r}\text { Least } \\
\text { Sq Mean }\end{array}$ \\
\hline 48 Hours,Positive Control 0.5\% A & & 91.419731 \\
24 Hours,Positive Control 1\% & A B & & 77.668002 \\
48 Hours,Positive Control 1\% & A B C & & 70.080334 \\
48 Hours,Salt 50\% Dilution & A B & & 69.059898 \\
24 Hours,Positive Control 0.5\% A B C D & 61.713125 \\
48 Hours,Salt No Dilution & A B C D & 56.264733 \\
24 Hours,Salt No Dilution & B C D E & 42.042789 \\
24 Hours,Salt 50\% Dilution & B C D E & 35.535787 \\
48 Hours,Normal No Dilution & C D E & 32.492856 \\
48 Hours,Normal 50\% Dilution & C D E & 31.526323 \\
24 Hours,Normal 50\% Dilution & D E & 24.059359 \\
48 Hours,Negative Control & C D E & 22.616975 \\
24 Hours,Normal No Dilution & D E & 17.719454 \\
24 Hours,Negative Control & & E & 15.681349
\end{tabular}

\begin{tabular}{|l|r|r|r|}
\hline Level & & $\begin{array}{r}\text { Least } \\
\text { Sq Mean }\end{array}$ \\
\hline Positive Control 0.5\% & A & & 76.566428 \\
Positive Control 1\% & A & & 73.874168 \\
Salt 50\% Dilution & B & 51.680554 \\
Salt No Dilution & B & 49.202730 \\
Normal 50\% Dilution & C & 27.792841 \\
Normal No Dilution & C & 25.073006 \\
Negative Control & C & 19.956144
\end{tabular}

ANOVA for Caspase Activity after normalizing to negative control 


\begin{tabular}{|l|r|r|r|r|}
\hline \multicolumn{4}{|l|}{ Analysis of Variance } \\
Source & DF & $\begin{array}{r}\text { Sum of } \\
\text { Squares }\end{array}$ & Mean Square & F Ratio \\
\hline Model & 7 & 19395.360 & 2770.77 & 0.7845 \\
Error & 8 & 28254.009 & 3531.75 & Prob > F \\
C. Total & 15 & 47649.369 & & 0.6190 \\
\hline
\end{tabular}

\section{Parameter Estimates}

\section{Term}

Intercept

Duration 2[24 Hours]

Group.[50\% Dilution]

Scaffold Type.[Normal]

Duration 2[24 Hours] *Group.[50\% Dilution]

Duration 2[24 Hours] *Scaffold Type.[Normal]

Group.[50\% Dilution]*Scaffold Type.[Normal]

Duration 2[24 Hours] ${ }^{*}$ Group.[50\% Dilution] ${ }^{*}$ Scaffold Type.[Normal]

\begin{tabular}{rrrr} 
Estimate & Std Error & t Ratio & Prob $>|\mathbf{t}|$ \\
\hline 37.229336 & 14.85713 & 2.51 & $0.0366^{*}$ \\
-13.64566 & 14.85713 & -0.92 & 0.3852 \\
2.677638 & 14.85713 & 0.18 & 0.8615 \\
-19.41099 & 14.85713 & -1.31 & 0.2277 \\
4.302638 & 14.85713 & 0.29 & 0.7795 \\
22.964011 & 14.85713 & 1.55 & 0.1608 \\
1.3406457 & 14.85713 & 0.09 & 0.9303 \\
9.7156457 & 14.85713 & 0.65 & 0.5315
\end{tabular}

ANOVA for Propidium Iodide Activity without normalizing to negative control

\begin{tabular}{|l|r|r|r|r|}
\hline \multicolumn{3}{|l|}{ Analysis of Variance } \\
\hline Source & DF & $\begin{array}{r}\text { Sum of } \\
\text { Squares }\end{array}$ & Mean Square & F Ratio \\
\hline Model & 13 & 43554.082 & 3350.31 & 11.2222 \\
\hline Error & 73 & 21793.654 & 298.54 & Prob > F \\
C. Total & 86 & 65347.737 & & $<.0001^{\star}$ \\
\hline
\end{tabular}

\begin{tabular}{|c|c|c|c|c|c|c|}
\hline \multicolumn{7}{|l|}{ Parameter Estimates } \\
\hline Term & Estimate & Std Error & t Ratio & Prob $>|t|$ & Lower $95 \%$ & Upper $95 \%$ \\
\hline Intercept & 25.123931 & 1.951242 & 12.88 & $<.0001^{*}$ & 21.235112 & 29.012751 \\
\hline Duration[24 Hours] & -20.08243 & 1.951242 & -10.29 & $<.0001 *$ & -23.97125 & -16.19361 \\
\hline Treatment[Negative Control] & -5.085755 & 5.101018 & -1.00 & 0.3221 & -15.25207 & 5.0805589 \\
\hline Treatment[Normal 50\% Dilution] & -4.75773 & 4.139465 & -1.15 & 0.2542 & -13.00767 & 3.492211 \\
\hline Treatment[Normal No Dilution] & -13.82881 & 4.51587 & -3.06 & $0.0031^{*}$ & -22.82892 & -4.828691 \\
\hline Treatment[Positive Control 0.5\%] & 10.676368 & 5.519329 & 1.93 & 0.0569 & -0.323638 & 21.676374 \\
\hline Treatment[Positive Control 1\%] & 11.523457 & 5.519329 & 2.09 & $0.0403^{*}$ & 0.5234507 & 22.523463 \\
\hline Treatment[Salt 50\% Dilution] & 9.5481082 & 4.252899 & 2.25 & $0.0278^{*}$ & 1.0720918 & 18.024125 \\
\hline Duration [24 Hours]*Treatment[Negative Control] & 10.155419 & 5.101018 & 1.99 & 0.0502 & -0.010895 & 20.321732 \\
\hline Duration[24 Hours] ${ }^{*}$ Treatment[Normal 50\% Dilution] & 5.3469688 & 4.139465 & 1.29 & 0.2005 & -2.902972 & 13.59691 \\
\hline Duration[24 Hours] ${ }^{*}$ Treatment[Normal No Dilution] & 10.193553 & 4.51587 & 2.26 & $0.0270^{*}$ & 1.1934376 & 19.193668 \\
\hline Duration[24 Hours] ${ }^{*}$ Treatment[Positive Control $\left.0.5 \%\right]$ & -13.36712 & 5.519329 & -2.42 & $0.0179 *$ & -24.36713 & -2.367118 \\
\hline Duration[24 Hours] ${ }^{*}$ Treatment[Positive Control 1\%] & -13.03184 & 5.519329 & -2.36 & $0.0209^{*}$ & -24.03184 & -2.031829 \\
\hline Duration [24 Hours]*Treatment[Salt 50\% Dilution] & -7.451075 & 4.252899 & -1.75 & 0.0840 & -15.92709 & 1.0249415 \\
\hline
\end{tabular}




\begin{tabular}{l|l|r|r}
\hline Level & & $\begin{array}{r}\text { Least } \\
\text { Sq Mean }\end{array}$ \\
\hline 48 Hours,Positive Control 1\% & A & & 69.761651 \\
48 Hours,Positive Control 0.5\% A & & 69.249851 \\
48 Hours,Salt 50\% Dilution & A & 62.205542 \\
48 Hours,Normal 50\% Dilution A B & 35.101660 \\
48 Hours,Negative Control & A B C & 29.965186 \\
48 Hours,Salt No Dilution & B C & 28.976623 \\
48 Hours,Normal No Dilution & B C & 21.184000 \\
24 Hours,Negative Control & B C & 10.111167 \\
24 Hours,Salt 50\% Dilution & B C & 7.138537 \\
24 Hours,Normal 50\% Dilution & B C & 5.630743 \\
24 Hours,Salt No Dilution & C & 5.119955 \\
24 Hours,Positive Control 1\% & B C & 3.533125 \\
24 Hours,Positive Control 0.5\% & B C & 2.350748 \\
24 Hours,Normal No Dilution & B C & 1.406250
\end{tabular}

ANOVA for Propidium Iodide Activity after normalizing to negative control

\begin{tabular}{|l|r|r|r|r|}
\hline \multicolumn{4}{|l|}{ Analysis of Variance } \\
Source & DF & $\begin{array}{r}\text { Sum of } \\
\text { Squares }\end{array}$ & Mean Square & F Ratio \\
\hline Model & 7 & 2605.0279 & 372.147 & 13.2244 \\
\hline Error & 8 & 225.1281 & 28.141 & Prob > F \\
C. Total & 15 & 2830.1560 & & $0.0008^{*}$
\end{tabular}

\section{Parameter Estimates}

\section{Term}

Intercept

Duration 2[24 Hours]

Group.[50\% Dilution]

Scaffold Type.[Normal]

Duration 2[24 Hours] ${ }^{*}$ Group.[50\% Dilution]

Duration 2[24 Hours] *Scaffold Type.[Normal]

Group.[50\% Dilution]*Scaffold Type.[Normal]

Duration 2[24 Hours] ${ }^{\star}$ Group.[50\% Dilution] ${ }^{\star}$ Scaffold Type.[Normal]

$\begin{array}{rrrr}\text { Estimate } & \text { Std Error } & \text { t Ratio } & \text { Prob }>|\mathbf{t}| \\ 4.7280343 & 1.326203 & 3.57 & 0.0073^{*} \\ -3.986104 & 1.326203 & -3.01 & 0.0169^{*} \\ 7.6524997 & 1.326203 & 5.77 & 0.0004^{*} \\ -5.339385 & 1.326203 & -4.03 & 0.0038^{*} \\ -6.072291 & 1.326203 & -4.58 & 0.0018^{*} \\ 4.6136641 & 1.326203 & 3.48 & 0.0083^{*} \\ -0.624687 & 1.326203 & -0.47 & 0.6502 \\ 1.1430565 & 1.326203 & 0.86 & 0.4138\end{array}$

\begin{tabular}{l|r|r|r|}
\hline Level & & $\begin{array}{r}\text { Least } \\
\text { Sq Mean }\end{array}$ \\
48 Hours,50\% Dilution,Salt & A & & 34.15972 \\
48 Hours,50\% Dilution,Normal & B & 10.71814 \\
48 Hours,No Dilution,Salt & B C & 3.17465 \\
24 Hours,50\% Dilution,Salt & B C & 2.52949 \\
24 Hours,50\% Dilution,Normal & B C & 2.11479 \\
24 Hours,No Dilution,Salt & B C & 0.40581 \\
24 Hours,No Dilution,Normal & B C & -2.08237 \\
48 Hours,No Dilution,Normal & C & -13.19596
\end{tabular}




\section{Appendix U: Statistical Tests for Scaffold Adhesion Assay}

ANOVA for Cell Coverage

\begin{tabular}{|l|r|r|r|r|}
\hline \multicolumn{5}{|l|}{ Analysis of Variance } \\
Source & DF & $\begin{array}{r}\text { Sum of } \\
\text { Squares }\end{array}$ & Mean Square & F Ratio \\
\hline Model & 5 & 20465.166 & 4093.03 & 17.6905 \\
Error & 18 & 4164.644 & 231.37 & Prob > F \\
C. Total & 23 & 24629.810 & & $<.0001^{\star}$ \\
\hline
\end{tabular}

\begin{tabular}{|l|rrrrrrr}
\hline | Parameter Estimates & & & & & & \\
\hline Term & Estimate & Std Error & t Ratio & Prob $>|\mathbf{t}|$ & Lower $95 \%$ & Upper 95\% \\
Intercept & 59.736792 & 3.104896 & 19.24 & $<.0001^{*}$ & 53.213648 & 66.259937 \\
Scaffold Type[Salt] & 27.842834 & 3.104896 & 8.97 & $<.0001^{*}$ & 21.319689 & 34.365978 \\
Location[Distal] & 2.3622627 & 4.390986 & 0.54 & 0.5972 & -6.862857 & 11.587382 \\
Location[Middle] & -4.283754 & 4.390986 & -0.98 & 0.3422 & -13.50887 & 4.9413656 \\
Scaffold Type[Salt]*Location[Distal] & 11.206408 & 4.390986 & 2.55 & $0.0200^{*}$ & 1.9812884 & 20.431527 \\
Scaffold Type[Salt]*Location[Middle] & -2.7329 & 4.390986 & -0.62 & 0.5415 & -11.95802 & 6.4922196
\end{tabular}

\begin{tabular}{l|r|r}
\hline & & $\begin{array}{r}\text { Least } \\
\text { Lq Mean }\end{array}$ \\
\hline Salt,Distal & A & 101.14830 \\
Salt,Proximal & A & 81.02761 \\
Salt,Middle & A & 80.56297 \\
Standard,Proximal & B & 42.28896 \\
Standard,Middle & B & 30.34310 \\
Standard,Distal & B & 23.04981
\end{tabular}

ANOVA for Percent Apoptotic Cells

\begin{tabular}{|l|r|r|r|r|}
\hline \multicolumn{4}{|l|}{ Analysis of Variance } \\
\hline Source & DF & $\begin{array}{r}\text { Sum of } \\
\text { Squares }\end{array}$ & Mean Square & F Ratio \\
\hline Model & 5 & 87.89023 & 17.5780 & 0.9983 \\
Error & 18 & 316.94525 & 17.6081 & Prob > F \\
C. Total & 23 & 404.83548 & & 0.4466 \\
\hline
\end{tabular}

I Parameter Estimates

\section{Term}

Intercept

Scaffold Type[Salt]

Location[Distal]

Location[Middle]

Scaffold Type[Salt]*Location[Distal]

Scaffold Type[Salt]*Location[Middle]

\section{Estimate}

14.442176

$-0.423776$

1.4972447

$-0.167609$

$-2.066265$

1.0859496

\section{Std Error t Ratio}

0.856545

0.856545

1.211338

1.211338

1.211338

1.211338
16.86

$-0.49$

1.24

$-0.14$

$-1.71$

0.90
Prob $>|t|$ Lower 95\%

$<.0001 \times$

0.6268

0.2323

0.8915

0.1052

0.3818
12.642641

$-2.223311$

$-1.047681$

$-2.712535$

$-4.611191$

$-1.458977$
Upper 95\%

16.24171

1.3757581

4.0421708

2.3773173

0.4786615

3.6308758 


\begin{tabular}{l||r|r}
\hline Level & & $\begin{array}{r}\text { Least } \\
\text { Sq Mean }\end{array}$ \\
\hline Standard,Distal & A & 18.429462 \\
Salt,Middle & A & 14.936740 \\
Salt,Proximal & A & 13.669078 \\
Standard,Middle & A & 13.612394 \\
Salt,Distal & A & 13.449379 \\
Standard,Proximal & A & 12.556001 \\
ANOVA for Percent Dead Cells
\end{tabular}

\begin{tabular}{|l|r|r|r|r|}
\hline \multicolumn{5}{|l|}{ Analysis of Variance } \\
Source & DF & $\begin{array}{r}\text { Sum of } \\
\text { Squares }\end{array}$ & Mean Square & F Ratio \\
\hline Model & 5 & 2097.5920 & 419.518 & 70.3957 \\
Error & 18 & 107.2697 & 5.959 & Prob > F \\
C. Total & 23 & 2204.8617 & & $<.0001^{*}$ \\
\hline
\end{tabular}

\section{| Parameter Estimates}

\begin{tabular}{|c|c|c|c|}
\hline \multicolumn{3}{|l|}{ Term } & Esti \\
\hline \multicolumn{3}{|l|}{ Intercept } & \\
\hline \multicolumn{3}{|l|}{ Scaffold Type[Salt] } & \\
\hline \multicolumn{3}{|l|}{ Location[Distal] } & \\
\hline \multicolumn{3}{|l|}{ Location[Middle] } & \\
\hline \multicolumn{4}{|c|}{ Scaffold Type[Salt]*Location[Distal] } \\
\hline \multicolumn{4}{|c|}{ Scaffold Type[Salt]*Location[Middle] } \\
\hline Level & & $\begin{array}{r}\text { Le } \\
\text { Sq Me }\end{array}$ & \\
\hline Salt,Distal & A & 22.4888 & \\
\hline Salt,Middle & A & 19.1225 & \\
\hline Salt,Proximal & A & 17.7013 & \\
\hline Standard,Proximal & B & 1.8042 & \\
\hline Standard,Middle & B & 1.5913 & \\
\hline Standard,Distal & B & 0.5257 & 772 \\
\hline
\end{tabular}




\section{Appendix V: Statistical Tests for BVM Study}

ANOVA for Change in Scaffold Thickness

\begin{tabular}{|l|r|r|r|r|}
\hline \multicolumn{4}{|c|}{ Analysis of Variance } & \\
\hline Source & DF & $\begin{array}{r}\text { Sum of } \\
\text { Squares }\end{array}$ & Mean Square & F Ratio \\
\hline Model & 5 & 0.37531111 & 0.075062 & 4.4709 \\
\hline Error & 12 & 0.20146667 & 0.016789 & Prob > F \\
C. Total & 17 & 0.57677778 & & $0.0156^{*}$ \\
\hline
\end{tabular}

\section{$\triangle$ Parameter Estimates}

\section{Term}

Intercept

Scaffold Type $[0.5 \%$ Salt]

Scaffold Type[3\% Salt]

Proximal/Distal[Distal]

Scaffold Type $\left[0.5 \%\right.$ Salt] ${ }^{*}$ Proximal/Distal[Distal]

Scaffold Type[3\% Salt]*Proximal/Distal[Distal]

$\begin{array}{rrrr}\text { Estimate } & \text { Std Error } & \text { t Ratio } & \text { Prob }>|\mathbf{t}| \\ 0.9211111 & 0.03054 & 30.16 & <.0001^{*} \\ 0.0422222 & 0.043191 & 0.98 & 0.3476 \\ 0.1388889 & 0.043191 & 3.22 & 0.0074^{\star} \\ 0.0477778 & 0.03054 & 1.56 & 0.1437 \\ 0.0222222 & 0.043191 & 0.51 & 0.6162 \\ 0.0122222 & 0.043191 & 0.28 & 0.7820\end{array}$

\begin{tabular}{|c|c|c|}
\hline Level & & $\begin{array}{l}\text { Least } \\
\text { Sq Mean }\end{array}$ \\
\hline $3 \%$ Salt,Distal & A & 1.1200000 \\
\hline $0.5 \%$ Salt,Distal & A & 1.0333333 \\
\hline $3 \%$ Salt,Proximal & A & 1.0000000 \\
\hline $0.5 \%$ Salt,Proximal & $A B$ & 0.8933333 \\
\hline Standard,Distal & B & 0.7533333 \\
\hline Standard,Proximal & B & 0.7266667 \\
\hline
\end{tabular}

ANOVA for Scaffold Shrinkage during pre-shrinking versus during BVM culture

\section{| Effect Tests}

\section{Source}

Scaffold Type.

Proximal/Distal 2

Preshrink/BVM

Scaffold Type.*Proximal/Distal 2

Scaffold Type.*Preshrink/BVM

Proximal/Distal 2*Preshrink/BVM

Scaffold Type.*Proximal/Distal 2*Preshrink/BVM

\section{Sum of}

$\begin{array}{rrrrrr}\text { Nparm } & \text { DF } & \text { Squares } & \text { F Ratio } & \text { Prob > F } \\ 1 & 1 & 9.59380 & 0.7144 & 0.4225 \\ 1 & 1 & 0.10057 & 0.0075 & 0.9332 \\ 1 & 1 & 837.92853 & 62.3981 & <.0001^{\star} \\ 1 & 1 & 17.52677 & 1.3052 & 0.2863 \\ 1 & 1 & 0.01142 & 0.0009 & 0.9774 \\ 1 & 1 & 0.00207 & 0.0002 & 0.9904 \\ 1 & 1 & 19.60393 & 1.4598 & 0.2615\end{array}$


ANOVA for Change in BVM Lengths

\begin{tabular}{|l|r|r|r|r|}
\hline \multicolumn{4}{|l|}{ Analysis of Variance } \\
Source & DF & $\begin{array}{r}\text { Sum of } \\
\text { Squares }\end{array}$ & Mean Square & F Ratio \\
\hline Model & 5 & 185.48956 & 37.0979 & 4.6198 \\
\hline Error & 6 & 48.18135 & 8.0302 & Prob > F \\
\hline C. Total & 11 & 233.67091 & & $0.0448^{*}$ \\
\hline
\end{tabular}

\begin{tabular}{|l|r|rrc|}
\hline Parameter Estimates & & & & \\
\hline Term & Estimate & Std Error & t Ratio & Prob $>|\mathbf{t}|$ \\
\hline Intercept & 3.370513 & 0.818038 & 4.12 & $0.0062^{*}$ \\
Scaffold Type[0.5\% Salt] & 1.6876265 & 1.15688 & 1.46 & 0.1949 \\
Scaffold Type[3\% Salt] & 3.1828865 & 1.15688 & 2.75 & $0.0332^{*}$ \\
Proximal/Distal[Distal] & 0.2658619 & 0.818038 & 0.32 & 0.7562 \\
Scaffold Type[0.5\% Salt]*Proximal/Distal[Distal] & 1.9783242 & 1.15688 & 1.71 & 0.1381 \\
Scaffold Type[3\% Salt]*Proximal/Distal[Distal] & -2.328741 & 1.15688 & -2.01 & 0.0908
\end{tabular}

\begin{tabular}{|l|r|r|}
\hline Level & & $\begin{array}{r}\text { Least } \\
\text { Sq Mean }\end{array}$ \\
\hline 3\% Salt A & 6.553400 \\
0.5\% Salt A & 5.058140 \\
Standard & B & -1.500000
\end{tabular}

ANOVA for Cell Coverage

\begin{tabular}{|l|r|r|r|r|}
\hline \multicolumn{5}{|l|}{ Analysis of Variance } \\
& Sum of & & \\
Source & DF & Squares & Mean Square & F Ratio \\
\hline Model & 7 & 66686.60 & 9526.66 & 14.6915 \\
Error & 148 & 95970.40 & 648.45 & Prob > F \\
C. Total & 155 & 162657.00 & & $<.0001^{*}$ \\
\hline
\end{tabular}

\begin{tabular}{|c|c|c|c|c|c|c|}
\hline \multicolumn{7}{|l|}{ Parameter Estimates } \\
\hline Term & Estimate & Std Error & t Ratio & Prob $>|t|$ & Lower $95 \%$ & Upper $95 \%$ \\
\hline Intercept & 41.043738 & 2.154957 & 19.05 & $<.0001^{*}$ & 36.78528 & 45.302196 \\
\hline Timepoint[3 Day] & 9.9654381 & 2.154957 & 4.62 & $<.0001^{*}$ & 5.70698 & 14.223896 \\
\hline Scaffold Type[0.5\% Salt] & 9.3992989 & 3.843936 & 2.45 & $0.0156^{*}$ & 1.8032096 & 16.995388 \\
\hline Scaffold Type[3\% Salt] & 21.213683 & 3.376168 & 6.28 & $<.0001^{*}$ & 14.541961 & 27.885404 \\
\hline Scaffold Type[ePTFE] & -9.950619 & 4.260655 & -2.34 & $0.0209^{*}$ & -18.3702 & -1.531042 \\
\hline Timepoint[3 Day]Sca & 3.112683 & 3.843936 & 0.81 & 0.4194 & -4.483406 & 10.708772 \\
\hline Timepoint[3 Day] ${ }^{\star S}$ & 0.7523346 & $3.37 €$ & 0.22 & 40 & -5.919387 & 7.4240563 \\
\hline Timepoint[3 Day]*Scaffold Type[ePTFE] & -2.58427 & 4.260655 & -0.61 & 0.5451 & -11.00385 & 5.8353071 \\
\hline
\end{tabular}




\begin{tabular}{l|l|l|r|}
\hline Level & & $\begin{array}{r}\text { Least } \\
\text { Sq Mean }\end{array}$ \\
\hline 3 Day,3\% Salt & A & & 72.975194 \\
3 Day,0.5\% Salt A B & & 63.521158 \\
7 Day,3\% Salt & A B C & 51.539648 \\
3 Day,ePTFE & B C D & 38.474288 \\
7 Day,0.5\% Salt & B C D & 37.364916 \\
3 Day,Standard & C D & 29.066066 \\
7 Day,ePTFE & D & 23.711951 \\
7 Day,Standard & D & 11.696685
\end{tabular}

ANOVA for Percent Apoptotic Cells

\begin{tabular}{|l|r|r|r|r|}
\hline \multicolumn{4}{|l|}{ Analysis of Variance } \\
Source & DF & $\begin{array}{r}\text { Sum of } \\
\text { Squares }\end{array}$ & Mean Square & F Ratio \\
\hline Model & 7 & 23055.601 & 3293.66 & 10.3093 \\
\hline Error & 148 & 47283.455 & 319.48 & Prob > F \\
C. Total & 155 & 70339.056 & & $<.0001^{\star}$
\end{tabular}

\section{Parameter Estimates}

\section{Term}

Intercept

Timepoint[3 Day]

Scaffold Type[0.5\% Salt]

Scaffold Type[3\% Salt]

Scaffold Type[ePTFE]

Timepoint[3 Day]*Scaffold Type[0.5\% Salt]

Timepoint[3 Day]*Scaffold Type[3\% Salt]

Timepoint[3 Day]*Scaffold Type[ePTFE]

\begin{tabular}{rrrrrrr} 
Estimate & Std Error & t Ratio & Prob $>|\mathbf{t}|$ & Lower 95\% & Upper 95\% \\
\hline 39.637433 & 1.512601 & 26.20 & $<.0001^{*}$ & 36.648347 & 42.626518 \\
-7.609559 & 1.512601 & -5.03 & $<.0001^{*}$ & -10.59864 & -4.620473 \\
4.5241166 & 2.698126 & 1.68 & 0.0957 & -0.80771 & 9.8559433 \\
6.2814726 & 2.369791 & 2.65 & $0.0089^{*}$ & 1.5984756 & 10.96447 \\
-15.39067 & 2.990628 & -5.15 & $<.0001^{\star}$ & -21.30052 & -9.480823 \\
-11.18964 & 2.698126 & -4.15 & $<.0001^{*}$ & -16.52147 & -5.857813 \\
1.605828 & 2.369791 & 0.68 & 0.4991 & -3.077169 & 6.288825 \\
7.3134086 & 2.990628 & 2.45 & $0.0156^{*}$ & 1.4035617 & 13.223255
\end{tabular}

\begin{tabular}{l||r|r|}
\hline Level & & $\begin{array}{r}\text { Least } \\
\text { Sq Mean }\end{array}$ \\
7 Day,0.5\% Salt A & & 62.960748 \\
7 Day,3\% Salt & A B & 51.922636 \\
7 Day,Standard A & B & 49.561668 \\
3 Day,3\% Salt & B C & 39.915175 \\
3 Day,Standard & B C & 38.883358 \\
3 Day,0.5\% Salt & C & 25.362351 \\
7 Day,ePTFE & C & 24.542913 \\
3 Day,ePTFE & C & 23.950613
\end{tabular}


ANOVA for Percent Dead Cells

\begin{tabular}{|l|r|r|r|r|}
\hline \multicolumn{4}{|l|}{ Analysis of Variance } \\
Source & DF & $\begin{array}{r}\text { Sum of } \\
\text { Squares }\end{array}$ & Mean Square & F Ratio \\
\hline Model & 7 & 7968.511 & 1138.36 & 10.8175 \\
\hline Error & 148 & 15574.502 & 105.23 & Prob > F \\
C. Total & 155 & 23543.013 & & $<.0001^{*}$ \\
\hline
\end{tabular}

\begin{tabular}{|c|c|c|c|c|c|c|c|c|}
\hline \multicolumn{9}{|c|}{ Parameter Estimates } \\
\hline \multicolumn{3}{|l|}{ Term } & Estimate & Std Error & t Ratio & Prob $>|t|$ & Lower $95 \%$ & Upper $95 \%$ \\
\hline \multicolumn{3}{|l|}{ Intercept } & 7.663905 & 0.868114 & 8.83 & $<.0001^{*}$ & 5.9484045 & 9.3794054 \\
\hline \multicolumn{3}{|l|}{ Timepoint[3 Day] } & -3.913307 & 0.868114 & -4.51 & $<.0001^{*}$ & -5.628807 & -2.197807 \\
\hline \multicolumn{3}{|c|}{ Scaffold Type[0.5\% Salt] } & -2.011364 & 1.548512 & -1.30 & 0.1960 & -5.071414 & 1.0486854 \\
\hline \multicolumn{3}{|c|}{ Scaffold Type[3\% Salt] } & 6.403738 & 1.360074 & 4.71 & $<.0001^{*}$ & 3.7160653 & 9.0914106 \\
\hline \multicolumn{3}{|c|}{ Scaffold Type[ePTFE] } & -1.055343 & 1.716386 & -0.61 & 0.5396 & -4.447131 & 2.3364453 \\
\hline \multicolumn{3}{|c|}{ Timepoint[3 Day]*Scaffold Type[0.5\% Salt] } & 1.4376381 & 1.548512 & 0.93 & 0.3547 & -1.622412 & 4.497688 \\
\hline \multicolumn{3}{|c|}{ Timepoint[3 Day] ${ }^{*}$ Scaffold Type[3\% Salt] } & -6.049475 & 1.360074 & -4.45 & $<.0001^{*}$ & -8.737148 & -3.361803 \\
\hline \multicolumn{3}{|c|}{ Timepoint[3 Day]*Scaffold Type[ePTFE] } & 1.1703028 & 1.716386 & 0.68 & 0.4964 & -2.221485 & 4.5620909 \\
\hline Level & & $\begin{array}{r}\text { Least } \\
\text { Sq Mean }\end{array}$ & & & & & & \\
\hline 7 Day,3\% Salt & $A$ & 24.030425 & & & & & & \\
\hline 7 Day,ePTFE & B & 9.351566 & & & & & & \\
\hline 7 Day, $0.5 \%$ Salt & B & 8.128209 & & & & & & \\
\hline 7 Day,Standard & B & 4.798647 & & & & & & \\
\hline 3 Day,3\% Salt & B & 4.104861 & & & & & & \\
\hline 3 Day,ePTFE & B & 3.865558 & & & & & & \\
\hline 3 Day,Standard & B & 3.855102 & & & & & & \\
\hline 3 Day, $0.5 \%$ Salt & B & 3.176872 & & & & & & \\
\hline
\end{tabular}

